\title{
Dynamic changes in cytochrome $c$ oxidase assembly and organization
}

\author{
Dissertation \\ for the award of the degree "Doctor rerum naturalium" \\ at the Georg-August-University Göttingen \\ within the doctoral program "Molecular Biology of Cells" \\ of the Georg-August University School of Science (GAUSS) \\ submitted by \\ Katharina Maria Römpler \\ from Lahnstein, Germany
}

Göttingen, 2016 

Members of the Thesis Committee

Prof. Dr. Peter Rehling

(Supervisor and first referee)

Prof. Dr. Heike Krebber

(Second referee)

Prof. Dr. Dörthe Katschinski
Institute for Cellular Biochemistry

University Medical Center

Göttingen, Germany

Department of Molecular Genetics

Institute for Microbiology and Genetics

Georg-August-University

Göttingen, Germany

Department of Cardiovascular Physiology

Center for Physiology and Pathophysiology

University Medical Center

Göttingen, Germany

\section{Additional Members of the Examination Board}

Prof. Dr. Markus Bohnsack

Prof. Dr. Stefan Jakobs

Prof. Dr. Michael Meinecke
Institute for Molecular Biology

University Medical Center

Göttingen, Germany

Department of Nanobiophotonics

Max Plack Institute for Biophysical Chemistry

Göttingen, Germany

European Neuroscience Institute

Göttingen, Germany

Date of oral examination: 17.08.2016 


\section{Affidavit}

I hereby declare that my dissertation, entitled "Dynamic changes in cytochrome $c$ oxidase assembly and organization", has been written independently and with no other aids or sources than quoted.

Katharina Römpler

Göttingen, June 2016 


\section{Table of contents}

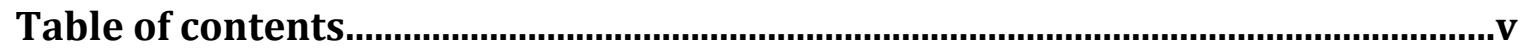

Publications............................................................................................................... viii

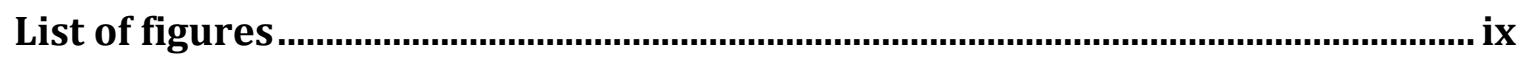

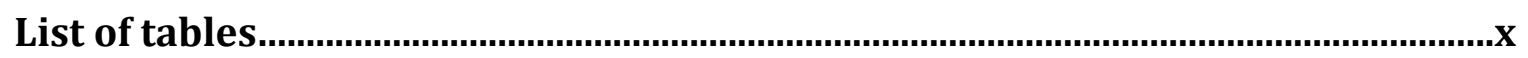

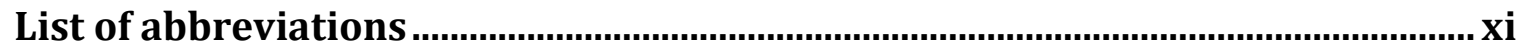

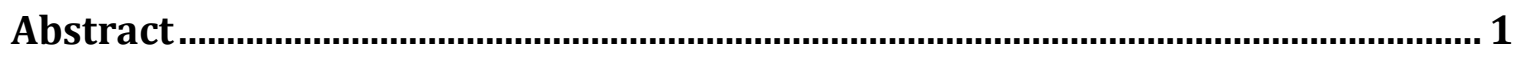

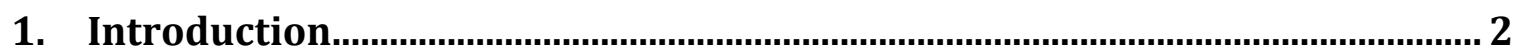

1.1. A brief history on mitochondria and their importance for eukaryotic life ....... 2

1.2. The bacterial heritage: Mitochondrial morphology and DNA ................................ 2

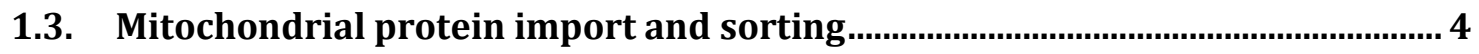

1.3.1. Proteins of the outer membrane and the IMS ……................................................. 5

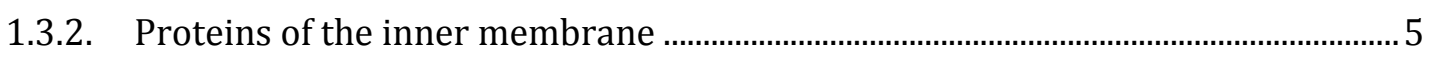

1.3.3. Soluble proteins of the matrix …….......................................................................... 7

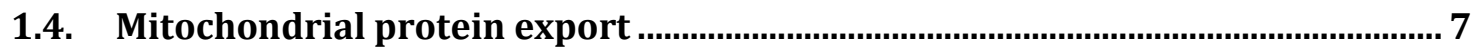

1.4.1. Conservative sorting of inner membrane proteins......................................................

1.4.2. Membrane insertion of mitochondrially encoded proteins ...................................... 8

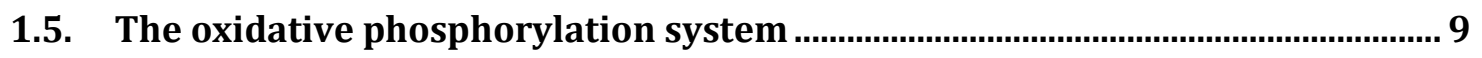

1.5.1. Composition and structure of complex III .......................................................... 10

1.5.2. Composition and structure of complex IV ............................................................ 11

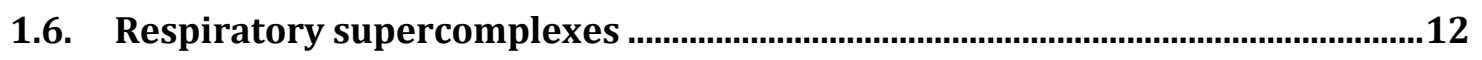

1.6.1. Organization of electron transport: solid versus fluid model ............................... 12

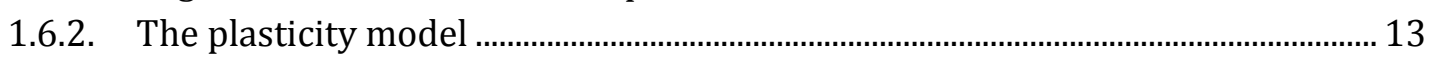

1.6.3. Physiological reasons for the formation of supercomplexes................................. 14

1.6.4. Supercomplex factors: Between assembly and stabilization................................... 15

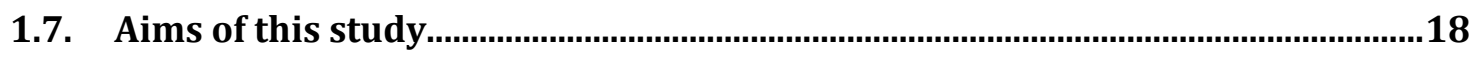

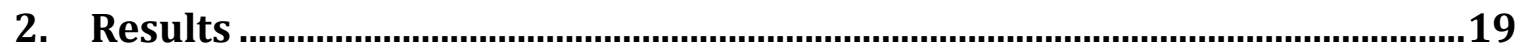

2.1. Maturation and assembly of the complex IV subunit Rcf2 …............................19

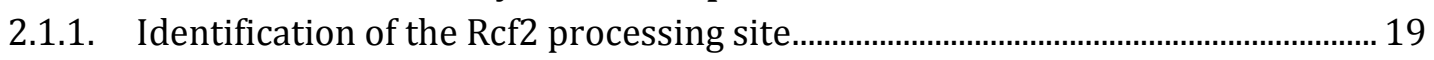

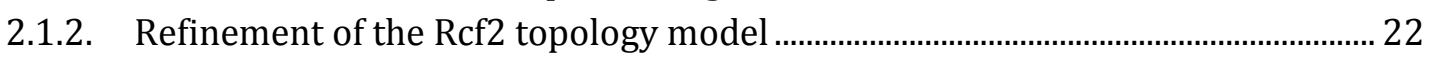

2.1.3. The role of intramembrane proteases in Rcf2 processing ..................................... 25

2.1.4. Rcf2C is associated with complex IV while Rcf2 ${ }^{\mathrm{N}}$ is not............................................. 26

2.1.5. Assembly of Rcf2 into supercomplexes depends on its C-terminus...................... 30

2.1.6. Rcf2 follows an unusual import pathway into the inner membrane..................... 32

2.2. YBR255C-A / Rcf3 is a novel interaction partner of complex IV..........................35

2.2.1. Rcf3 is a protein of the inner mitochondrial membrane ......................................... 36

2.2.2. Rcf3 is not essential for respiration but interacts with respiratory

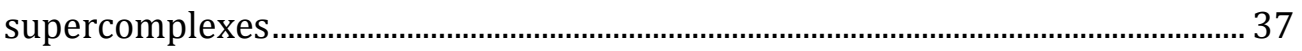

2.2.3. The supercomplex association of Rcf3 is mediated through complex IV and

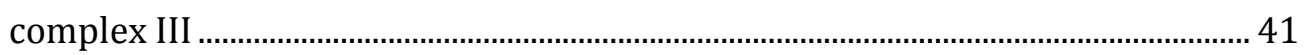

2.2.4. Rcf proteins accumulate in small complexes........................................................... 43

2.2.5. Attempts to define the role of the small Rcf-containing complexes ...................... 45

2.3. Investigation of a potential interplay of Rcf proteins........................................48

2.3.1. Overexpression of Rcf2 fragments or Rcf3 is harmless ......................................... 48 


\section{CONTENTS}

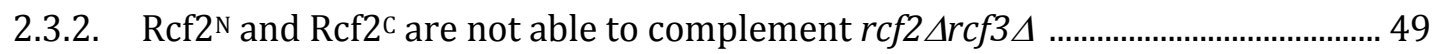

2.3.3. Attempts to define specific Rcf2 interaction partners in vivo ............................... 52

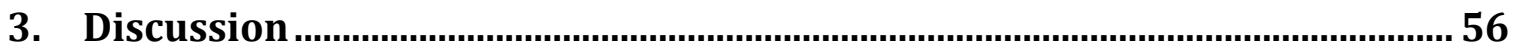

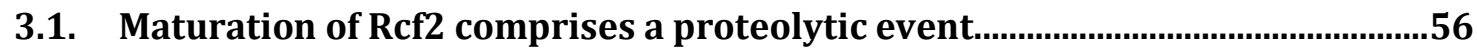

3.1.1. Limited proteolysis of Rcf2 by an unknown protease .............................................. 56

3.1.2. From regulation to degradation - the role of Rcf2 $2^{\mathrm{N}}$................................................. 57

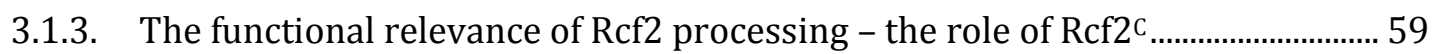

3.2. Import of Rcf2 might rely on TIM23 and OXA1

3.3. Rcf proteins reside at the interface of complex III and IV .................................62

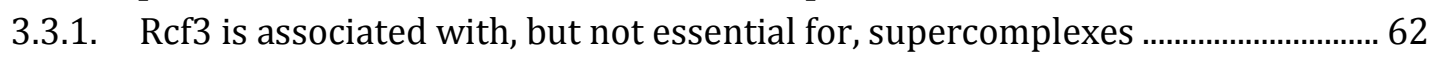

3.3.2. How do Rcf proteins fit in the current crystal structures? ...................................... 63

3.3.3. Complex IV* - reasons for an Rcf-specific version of complex IV .......................... 64

3.4. Small Rcf complexes could serve as an interaction platform .............................65

3.4.1. The importance of the Rcf3 C-terminus for small Rcf3 complexes........................ 65

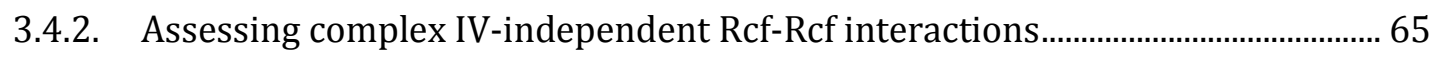

3.4.3. Connecting Rcf2 and Rcf3 - the split paralogue hypothesis.................................... 67

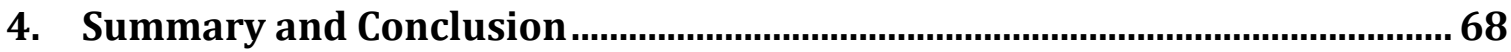

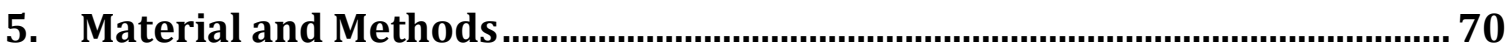

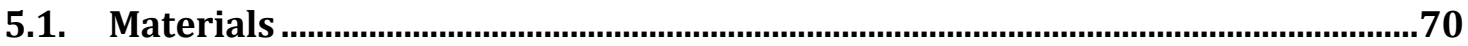

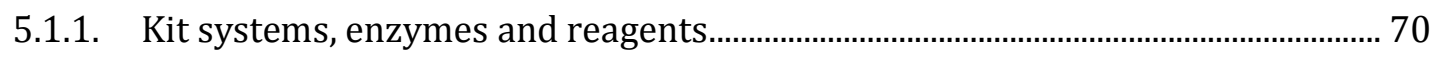

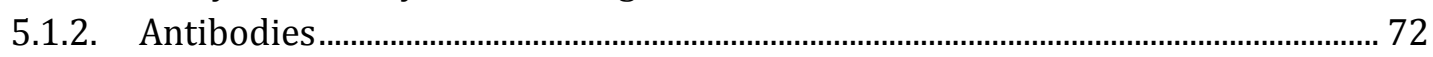

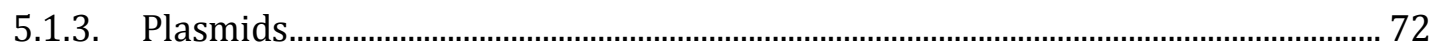

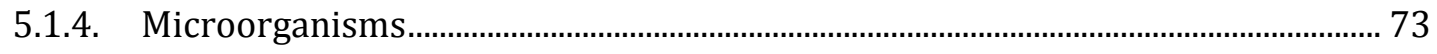

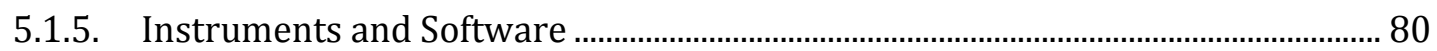

5.2. Cultivation and handling of microorganisms .....................................................81

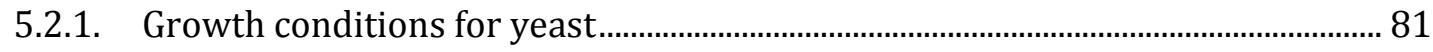

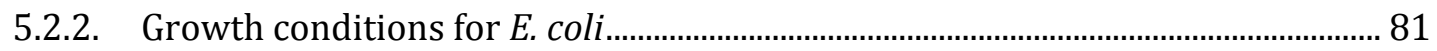

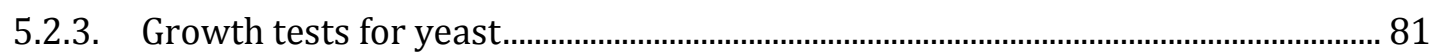

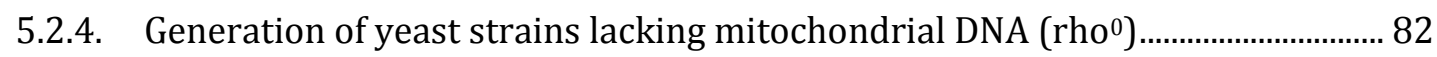

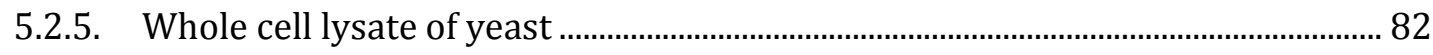

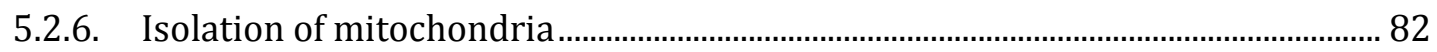

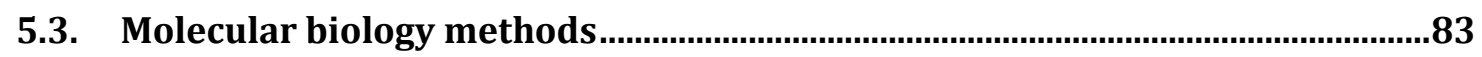

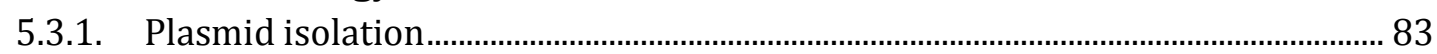

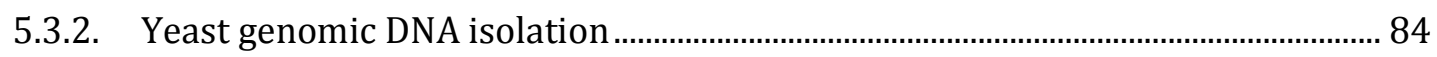

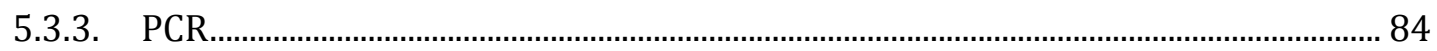

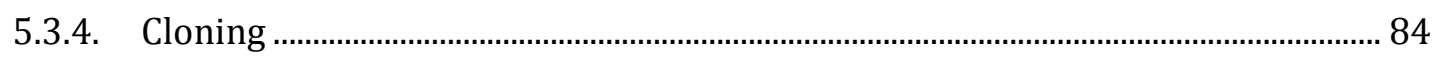

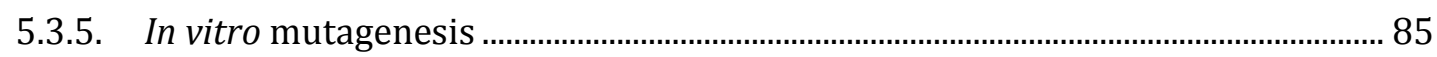

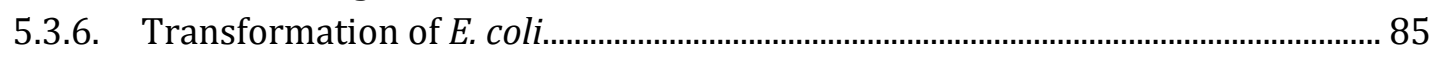

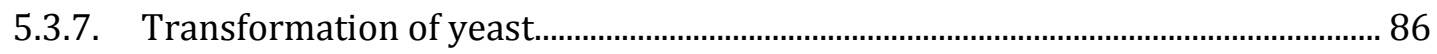

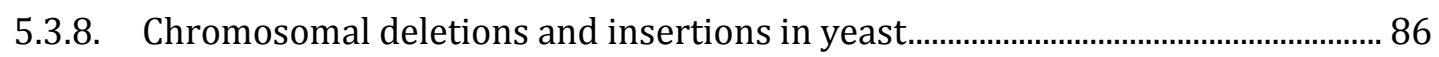

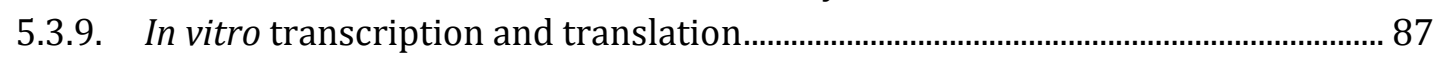

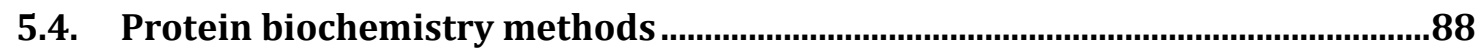

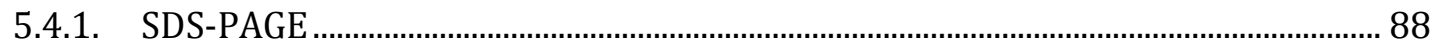

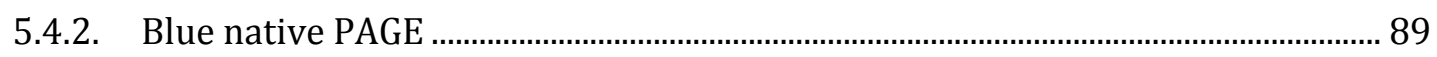

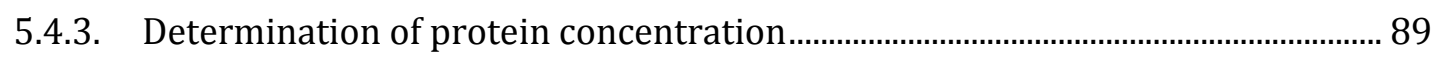




\section{CONTENTS}

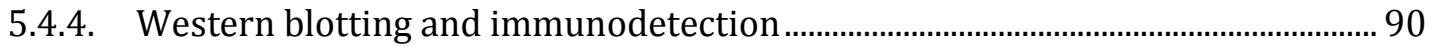

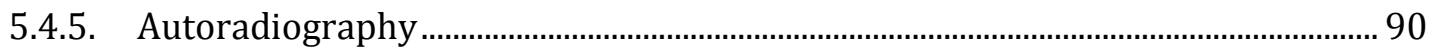

5.4.6. Coomassie staining of membranes and gels ............................................................ 91

5.4.7. Steady state analysis of mitochondrial proteins ....................................................... 91

5.4.8. Testing solubilization properties of mitochondria proteins.................................... 91

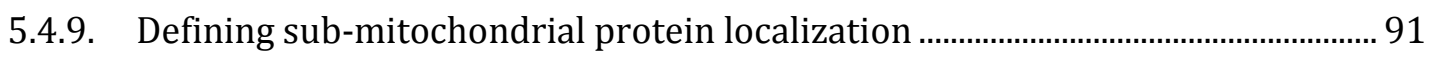

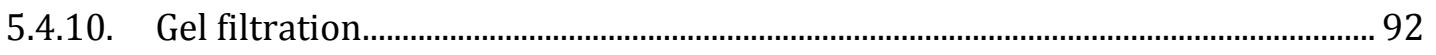

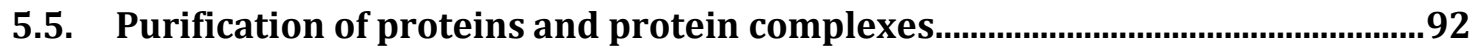

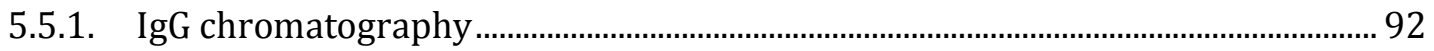

5.5.2. Crosslinking of antibodies to PA-Sepharose.............................................................. 93

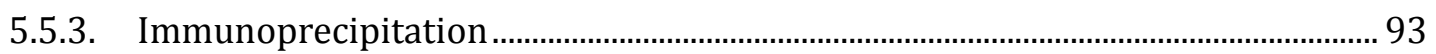

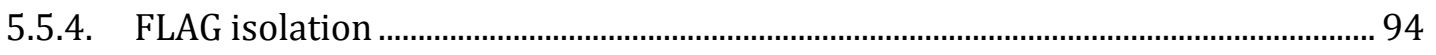

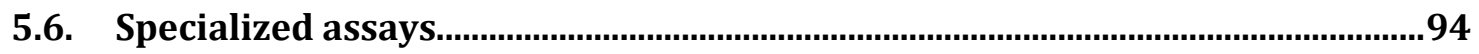

5.6.1. In vitro protein import and assembly assay ............................................................. 94

5.6.2. Modification of cysteines using PEG maleimid......................................................... 95

5.6.3. Protease inhibitor treatment of mitochondria ………………………….................. 95

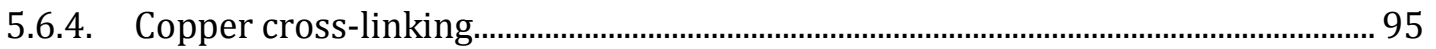

5.6.5. Determination of enzyme activities in vitro............................................................. 96

5.6.6. Determination of oxygen consumption rates.......................................................... 96

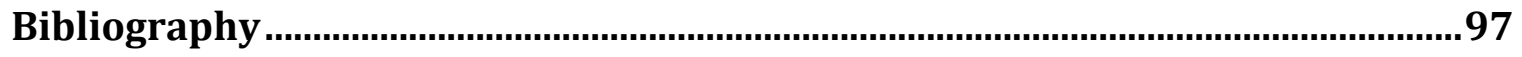

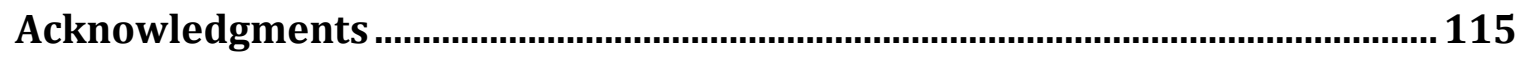




\section{Publications}

Parts of this thesis will be published in:

Römpler, K., Juris, L., Wissel, M., Vukotic, M., Hofmann, K., \& Deckers, M. (currently under revision) The Rcf2 homologue Rcf3 associates with respiratory chain supercomplexes. The Journal of Biological Chemistry. 


\section{List of figures}

Figure 1-1: Schematic representation of a mitochondrial cross-section........................................................... 3

Figure 1-2: Overview of the import pathways into different mitochondrial compartments...........................5

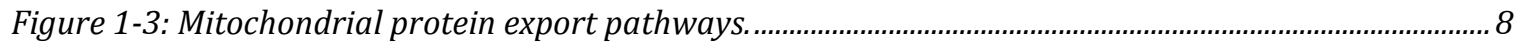

Figure 1-4: Model of the respiratory chain in mammals and yeast................................................................ 10

Figure 1-5: Schematic presentation of fluid and solid view of respiratory chain organization................... 13

Figure 1-6: Schematic representation of the plasticity model......................................................................... 14

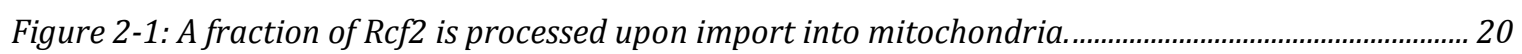

Figure 2-2: FLAG Rcf2 localizes to mitochondria and enables detection of $R c f 2^{N}$ and $R c f 2^{C}$........................... 21

Figure 2-3: Rcf2 exhibits a four TMD conformation with its $N$ - and $C$-terminus facing the IMS................. 23

Figure 2-4: Deletion of PCP1 and treatment with common protease inhibitors do not affect the

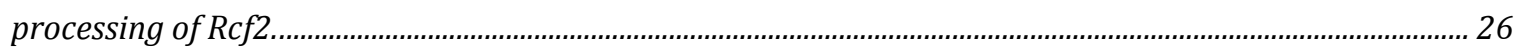

Figure 2-5: Rcf2C comigrates with and is coisolated by complex IV and respiratory supercomplexes....... 27

Figure 2-6: $R c f 2^{C}$ is associated with complex IV*, a specific population of complex IV................................ 28

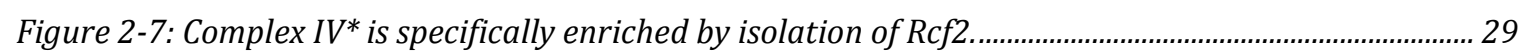

Figure 2-8: ${ }^{F L A G R} R 2^{N}$ is highly unstable and does not associate with any of the Rcf2-containing

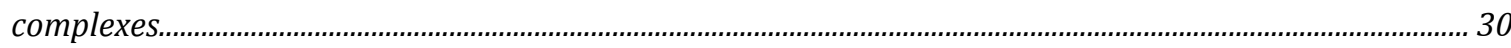

Figure 2-9: The C-terminal half of Rcf2 present in $R c f 2^{C}$ is essential for Rcf2 assembly into

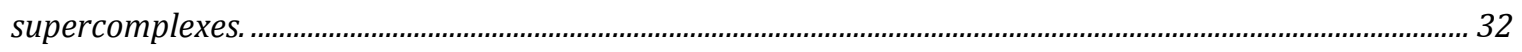

Figure 2-10: Import of Rcf2 does not rely on a functional carrier pathway................................................. 33

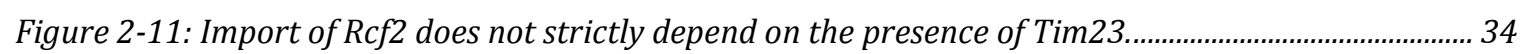

Figure 2-12: Alignment visualizing sequence similarities among Rcf1, Rcf2 and YBR255C-A (Rcf3)....... 35

Figure 2-13: Rcf3 localizes to the inner mitochondrial membrane and exposes its $C$-terminus to the IMS.

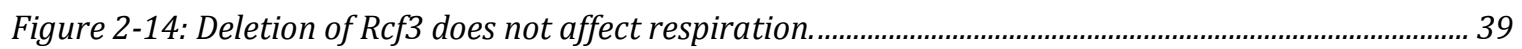

Figure 2-15: Rcf3 is assembled into respiratory supercomplexes in isolated rcf3 $\triangle$ mitochondria.............. 40

Figure 2-16: Endogenous Rcf3 interacts with supercomplexes but dissociates in DDM. ............................. 41

Figure 2-17: C-terminal ZZ tagging alters supercomplex association of Rcf3 even though respiration

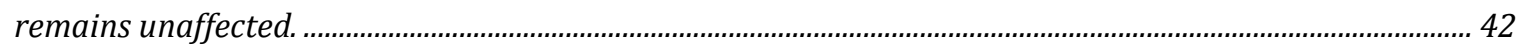

Figure 2-18: Rcf3 is able to interact with both complex III and complex IV................................................ 43

Figure 2-19: All Rcf proteins accumulate in small assemblies independent of complex III / IV.................. 44

Figure 2-20: Rcf proteins interact with each other independently of complex III or IV................................ 46

Figure 2-21: The small Rcf complexes form independently of the presence of the other RcfS.................... 47

Figure 2-22: Respiration is not affected by overexpression of $R c f 3, \operatorname{Rcf} 2^{N}$ or $R c f 2^{C}$..................................... 49

Figure 2-23: Double deletion of RCF2 and RCF3 generates a strain impaired in respiration...................... 50

Figure 2-24: Untagged Rcf2 and Rcf3 can complement $r c f 2 \Delta r c f 3 \Delta$, while $R c f 2^{N}$ and Rcf2 ${ }^{C}$ are nonfunctional.

Figure 2-25: All cysteine mutant versions of Rcf2 are functional.. 52 
Figure 2-26: Insertion of cysteines at different positions leads to specific changes in the cross-linking pattern.

Figure 2-27: Two-step purification after copper cross-linking enriches a 70 kDa cross-link in wild-type.

Figure 2-28: The 70 kDa cross-link is unlikely to contain another subunit of complex IV...........................55

Figure 3-1: Cleavage of Rcf2 and possible fates of the resulting fragments, $R c f 2^{N}$ and $R c f 2^{C}$......................58

Figure 3-2: Hypothetical import pathway of Rcf2, through the combined action of TIM23SORT,

\section{List of tables}

Table 5-1: Kit systems and enzymes used in this study 70

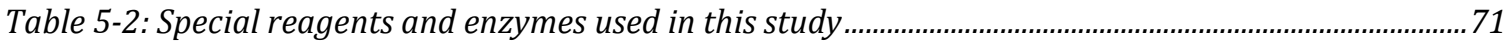

Table 5-3: Commercially available antibodies used in this study ..................................................................... 72

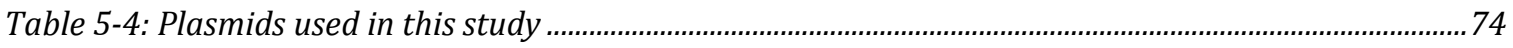

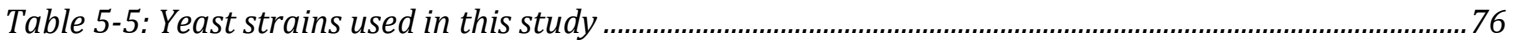

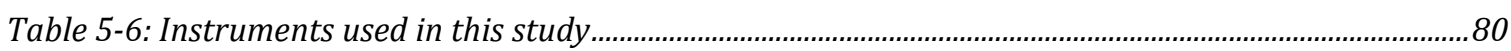

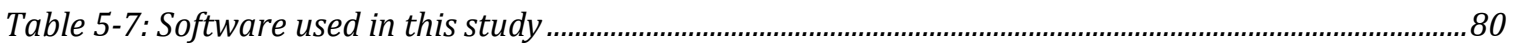

Table 5-8: Conditions for Flexi ${ }^{\circledR}$ Rabbit Reticulocyte Lysate System ……......................................................8 


\section{List of abbreviations}

\begin{tabular}{|c|c|}
\hline$\downarrow$ & down regulation \\
\hline$\uparrow$ & up regulation \\
\hline$\Delta \Psi$ & Membrane potential across the inner membrane \\
\hline AAC & ADP/ATP carrier \\
\hline $\mathrm{ADP}$ & Adenosine diphosphate \\
\hline APS & Ammonium persulfate \\
\hline ATP & Adenosine triphosphate \\
\hline AVO & Antimycin A, valinomycin, oligomycin mixture \\
\hline BN-PAGE & Blue native polyacrylamide gel electrophoresis \\
\hline BSA & Bovine serum albumin \\
\hline CSM & Complete supplement mixture \\
\hline DHFR & Mouse dihydrofolate reductase \\
\hline DMP & Dimethy pimelimidate dihydrochloride \\
\hline DMSO & Dimethyl sulfoxide \\
\hline DTT & Dithiotreitol \\
\hline $\mathrm{E}$ & Eluate \\
\hline ECL & Enhanced chemiluminescence \\
\hline EDTA & Ethylenediaminetetraacetic acid \\
\hline ER & Endoplasmic reticulum \\
\hline GFP & Green fluorescent protein \\
\hline HEPES & 4-(2-hydroxyethyl)-1-piperazineethanesulfonic acid \\
\hline HRP & Horse radish peroxidase \\
\hline IM & Inner mitochondrial membrane \\
\hline IMP & Inner membrane protease \\
\hline IMS & Intermembrane space \\
\hline LB & Lysogeny broth \\
\hline MIA & Mitochondrial IMS assembly machinery \\
\hline MIM & Mitochondrial import complex \\
\hline MIP & Mitochondrial intermediate peptidase \\
\hline MOPS & 3-(N-morpholino)propanesulfonic acid \\
\hline MPP & Mitochondrial processing peptidase \\
\hline mtDNA & Mitochondria DNA \\
\hline mt ribosome & Mitochondrial ribosome \\
\hline NADH & Nicotinamide adenine dinucleotide \\
\hline $\mathrm{OM}$ & Outer mitochondrial membrane \\
\hline ORF & Open reading frame \\
\hline OXA & Export and assembly machinery of the inner membrane \\
\hline PAM & Presequence translocase-associated motor \\
\hline PBS & Phosphate buffered saline \\
\hline PEG & Polyethylene glycol \\
\hline
\end{tabular}


Proteinase K

PMSF Phenylmethylsulfonyluoride

PVDF Polyvinylideneuoride

rho $^{0} \quad$ lacking mitochondrial DNA

ROS Reactive oxygen species

rRNA ribosomal RNA

SAM Sorting and assembly machinery

SF Tag combining Streptavidine and Flag peptide

$\mathrm{SD} / \mathrm{G} / \mathrm{Lac} \quad$ Selective medium containing glucose/ glycerol/galactose/ lactate

Su9 subunit 9 of the ATP synthase

TAE Tris, acetic acid, EDTA

TBS-T Tris buffered saline with Tween 20

TCA Trichloroacetic acid

TE Tris, EDTA

TEMED N,N,N',N'-tetramethylethylenediamine

TEV Tobacco Etch Virus protease

TIM22 Carrier translocase of the inner membrane

TIM23 Presequence translocase of the inner membrane

TMD Transmembrane domain

tRNA transfer RNA

$\mathrm{T} \quad$ Total

TOM Translocase of the outer membrane

UB Unbound

YPD/G/Gal Yeast extract, peptone, glucose/ glycerol/galactose

$\mathrm{ZZ}$

Tag combining Protein A, His and a TEV cleavage site 


\section{Abstract}

The respiratory chain in the inner membrane of the yeast mitochondrion is organized as a network of individual complexes and large supercomplex structures. These supercomplexes are composed of dimeric complex III and one or two copies of complex IV ( $\mathrm{III}_{2} \mathrm{IV}$ and $\left.\mathrm{III}_{2} \mathrm{IV}_{2}\right)$. Even though the existence of respiratory supercomplexes has been shown for a variety of organisms, it is not fully understood which purpose they serve and how they are assembled as well as regulated. Lipids, protein complexes and single proteins were proposed to take part in these intricate processes. To the latter group of potential supercomplex regulators belongs the Rcf protein family which is composed of three related proteins: Rcf1, Rcf2 and the so far uncharacterized YBR255C-A/ Rcf3. Rcf1 was shown to be essential for the formation of $\mathrm{III}_{2} \mathrm{IV}_{2}$. To obtain a deeper understanding of the role of the Rcf protein family in supercomplex formation and stability, this study aimed at an in-depth investigation of Rcf2 and Rcf3.

Like Rcf1 and Rcf2, Rcf3 proved to be a constituent of supercomplexes via its association with complex IV*, an Rcf-specific version of complex IV. All three Rcfs furthermore revealed the ability to interact with complex III in the absence of complex IV, positioning them at the interface of both complexes. In contrast to Rcf1, Rcf3 and Rcf2 are dispensable for supercomplex formation. However, despite unchanged supercomplex organization, simultaneous deletion of RCF2 and RCF3 leads to severely reduced respiratory growth. This indicates a functional overlap, which is further supported by the sequence similarities of Rcf3 with the N-terminus of Rcf2 and the observed processing of Rcf2. This study revealed that Rcf2 is subjected to limited proteolysis after import into mitochondria. The resulting $\mathrm{N}$-terminal fragment, Rcf2 $\mathrm{N}$, was neither observed in individual complexes nor in supercomplexes. Whether it is degraded or preserved to fulfill a regulatory function within the respiratory chain could not be clarified on the basis of the present data. In contrast, the C-terminal fragment, $\mathrm{Rcf}^{\mathrm{C}}$, is assembled into complex IV* along with the remaining full-length Rcf2. It was hence found in supercomplexes. Further investigations will elucidate its function and the significance of the processing event in regard to supercomplex organization. 


\section{Introduction}

\subsection{A brief history on mitochondria and their importance for eukaryotic life}

The establishment of intracellular membrane-enclosed compartments is one of the hallmarks that distinguish eukaryotes from prokaryotes. The first of these membrane-enclosed organelles, the nucleus, was identified as early as 1719 , but also mitochondria had already been known since the 1840s. When in 1894 Altmann named them "bioblasts" (Altmann, 1894) he probably did not anticipate the immensely growing attention they received over the following 120 years. He nevertheless demonstrated a remarkable vision when he proposed them to be organisms that live inside the cell to fulfill vital functions. Today, the generally accepted endosymbiotic theory in fact states the engulfment of an $\alpha$-proteobacterium by a so far unidentified host as the origin of mitochondria (Andersson \& Kurland, 1998; Margulis, 1970; Szklarczyk \& Huynen, 2010). Several features of modern mitochondria can be traced back to this endosymbiosis: among others, the double membrane, the autonomously replicated mitochondrial genome and the striking similarities of mitochondrial and bacterial translation machineries. After receiving their name in 1898 (Benda, 1898), step by step, mitochondria were shown to be essential for several metabolic pathways of eukaryotic cells. They contain the Krebs cycle and the OXPHOS system and decisively contribute to the $\beta$-oxidation of fatty acids and the biogenesis of amino acids and iron sulfur clusters (Ernster \& Schatz, 1981; Lill et al., 2012). They furthermore play a role in apoptosis (Green \& Reed, 1998) and in cellular calcium homeostasis (Rimessi, Giorgi, Pinton, \& Rizzuto, 2008). Through the ERMES complex, mitochondria establish contact sites with the endoplasmic reticulum (ER) that are important for interorganellar lipid exchange (Rowland \& Voeltz, 2012). Thus, mitochondria are essential organelles not only for respiring cells.

\subsection{The bacterial heritage: Mitochondrial morphology and DNA}

The double membrane, a consequence of the endosymbiotic event, renders mitochondria highly compartmentalized (Figure 1-1). The outer membrane (OM) 
separates the organelle from the cytoplasm and represents a barrier for large molecules but enables diffusion of ions and small metabolites through large protein pores (Benz, 1994). The inner membrane (IM) is a tightly sealed barrier between the intermembrane space (IMS) and the mitochondrial matrix. Ions, metabolites and proteins cross the IM through specific and regulated transporters, carriers or translocases. IM and IMS are further compartmentalized by invaginations of the IM, so called cristae. Mainly two large protein complexes determine the cristae shape. The MICOS complex induces membrane curvature and the formation of cristae junctions at the proximal end (Barbot et al., 2015; van der Laan, Bohnert, Wiedemann, \& Pfanner, 2012), while dimerization of the ATP synthase stabilizes the distal end (Paumard et al., 2002). Hence, the IM is structured into three different sections: cristae membrane, cristae junction and inner boundary membrane.

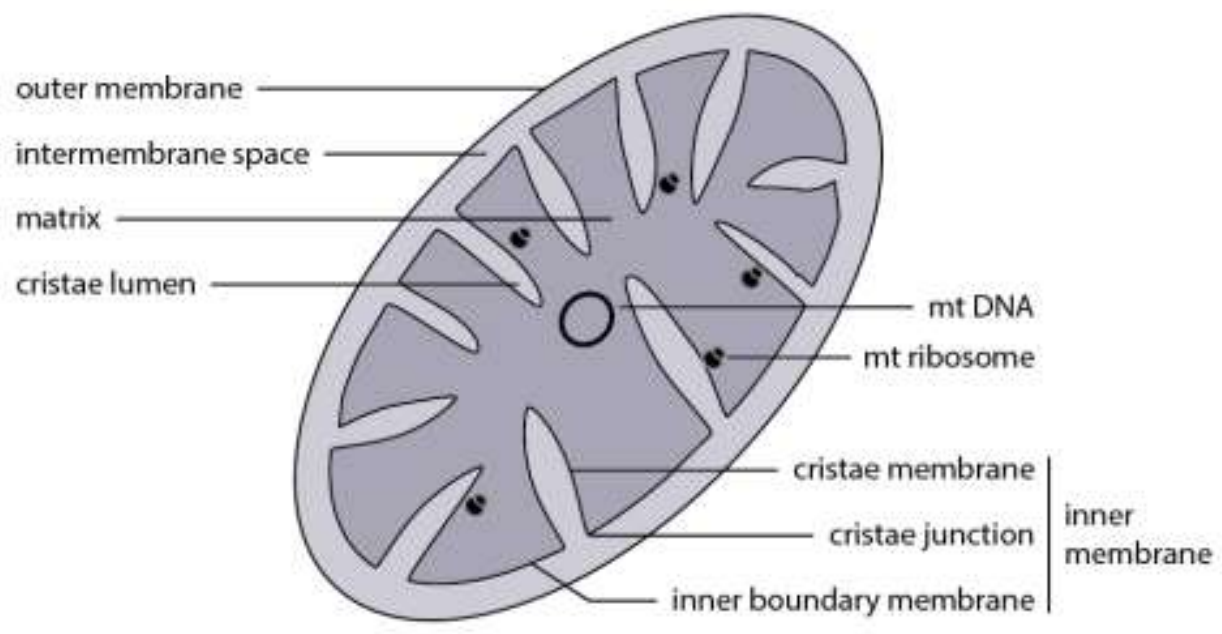

Figure 1-1: Schematic representation of a mitochondrial cross-section. The outer membrane encloses the intermembrane space (including the cristae lumen), the inner membrane and the matrix. The inner membrane is structured into inner boundary membrane, cristae junction regions and cristae membranes. The matrix contains the mitochondrial genome and the inner membraneattached mitochondrial ribosomes.

In contrast to the old textbook picture of a small bean-shaped organelle, in most cells, mitochondria exist as a dynamic network (Friedman \& Nunnari, 2014). The mitochondrial network undergoes fission and fusion events on a regular basis in order to answer the cells metabolic demands, to separate damaged parts for mitophagy (Müller, Lu, \& Reichert, 2015) but also to distribute mitochondria during cell division (Mishra \& Chan, 2014).

A prerequisite for this mitochondrial remodeling is a constant biogenesis of mitochondrial proteins. As mentioned, mitochondria still contain their own genome 


\section{INTRODUCTION}

that is replicated and transcribed in the matrix (Figure 1-1). During evolution, most of the original $\alpha$-proteobacterial DNA was lost or transferred to the nucleus (Gabaldón \& Huynen, 2004). As a result, the modern mitochondrial genome codes for eight proteins in yeast and thirteen in mammals. It additionally contains the information for transfer RNAs (tRNA) and ribosomal RNAs (rRNA) for the mitochondrial translation machinery. Mitochondrially encoded proteins are synthesized on membrane attached mitochondrial ribosomes. Most of them are highly hydrophobic proteins that are cotranslationally inserted into the IM (Ott \& Herrmann, 2010).

The yeast mitochondrial proteome in its entity was addressed in several proteomic studies and comprises around 1000 proteins (Hess et al., 2009; Prokisch et al., 2004; Sickmann et al., 2003). Hence more than $99 \%$ of mitochondrial proteins are encoded by the nuclear genome, synthesized on cytosolic ribosomes and post-translationally imported into their respective mitochondrial location.

\subsection{Mitochondrial protein import and sorting}

Apart from $\alpha$ helical proteins of the $\mathrm{OM}$, all nuclear encoded mitochondrial proteins are initially translocated across the OM with the help of the translocase of the outer membrane (TOM). The unfolded precursor proteins are chaperoned to the TOM complex and recognized by specific receptors (Dudek, Rehling, \& van der Laan, 2013). The majority of mitochondrial proteins contains an N-terminal targeting sequence that is organized as positively charged amphipatic helix of 15 to 50 residues, named presequence (Allison \& Schatz, 1986; Roise, Horvath, Tomich, Richards, \& Schatz, 1986; Vögtle et al., 2009). This presequence is usually removed by the mitochondrial processing peptidase (MPP). Some proteins are also processed by further peptidases like MIP (mitochondrial intermediate peptidase) or IMP (inner membrane protease) (Käser \& Langer, 2000; Koppen \& Langer, 2007). In addition, non-cleavable C-terminal or internal targeting sequences are described (Fölsch, Guiard, Neupert, \& Stuart, 1996; Kutik et al., 2008; C. M. Lee, Sedman, Neupert, \& Stuart, 1999; Reinhold et al., 2012). The TOM complex is composed of the pore forming central subunit Tom40 and several additional subunits that mediate precursor binding (reviewed in Bohnert, Pfanner, \& van der Laan, 2015; Dudek et al., 


\section{INTRODUCTION}

2013; Schulz, Schendzielorz, \& Rehling, 2015). After translocation across the OM, the downstream import pathway depends on the destination of the protein (Figure 1-2).

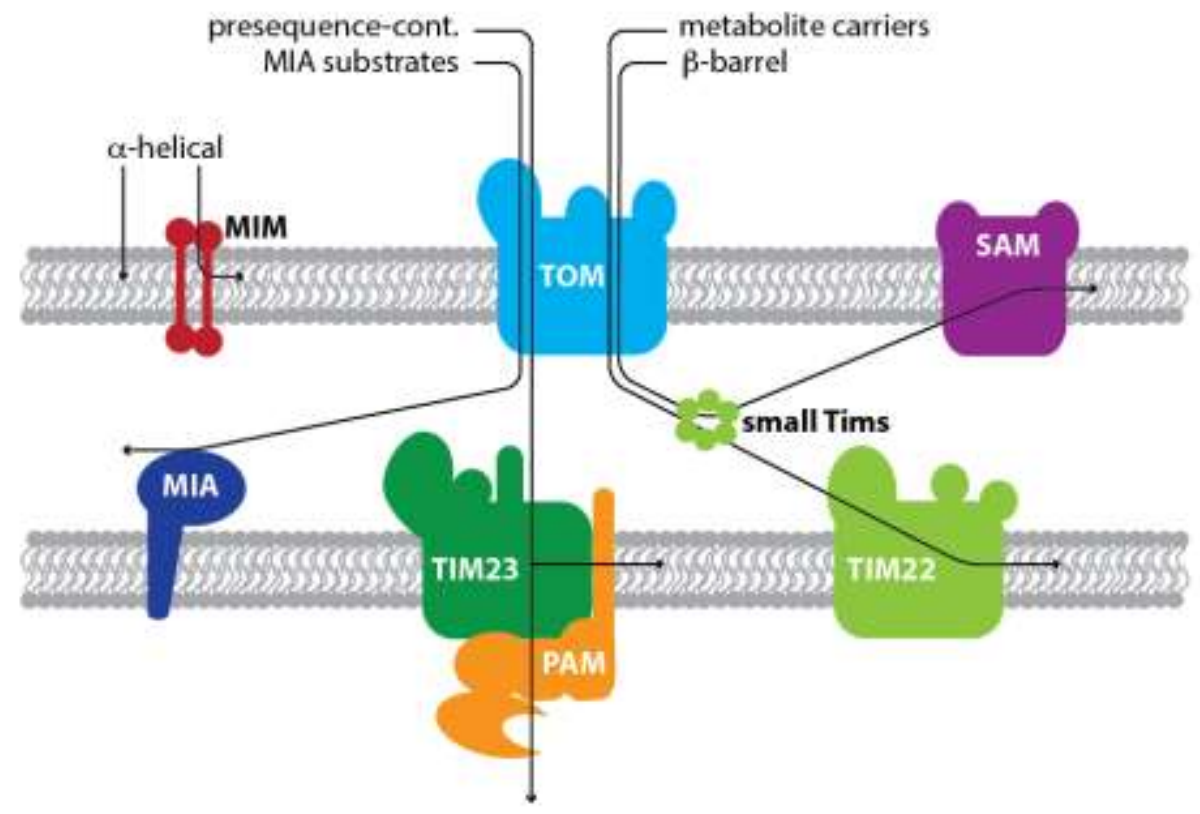

Figure 1-2: Overview of the import pathways into different mitochondrial compartments. Except for $\alpha$-helical OM proteins, all proteins are translocated across the OM with the help of TOM. $\beta$-barrel proteins of the OM are transferred via small Tims to SAM, which then mediates membrane insertion. $\alpha$-helical OM proteins are inserted via MIM or without the help of a proteinaceous machinery, Cystein-containing IMS proteins are trapped by oxidative folding by MIA. Carrier proteins of the IM are inserted through TIM22, while presequence-containing IM proteins are laterally released from TIM23. Matrix proteins are imported through coordinated action of TIM23 and PAM.

\subsubsection{Proteins of the outer membrane and the IMS}

Most OM proteins are $\beta$-barrel proteins that are inserted into the OM through the sorting and assembly machinery (SAM) after translocation into the IMS (reviewed in Bohnert et al., 2015; Dudek et al., 2013). $\alpha$-helical OM proteins do not rely on TOM and are inserted either through the mitochondrial import complex (MIM) (Becker et al., 2008; Dimmer et al., 2012) or without the help of a proteinaceous machinery (Kemper et al., 2008; Krumpe et al., 2012). Soluble cysteine-containing proteins are trapped by oxidative folding mediated by the mitochondrial intermembrane space import and assembly (MIA) machinery (Vögtle et al., 2012).

\subsubsection{Proteins of the inner membrane}

The inner membrane is densely packed with protein complexes, rendering it the most protein-rich compartment of mitochondria (Daum, Böhni, \& Schatz, 1982). Integral IM proteins differ in structure and topology and so do their respective 


\section{INTRODUCTION}

import pathways, even though all of them require a translocase of the inner membrane (TIM).

TIM22 mediated import: Metabolite carrier proteins are multispanning inner membrane proteins with six transmembrane domains (TMD), which overlap with the internal hydrophobic targeting signals (Brix, Rüdiger, Bukau, SchneiderMergener, \& Pfanner, 1999). These proteins are inserted into the IM with the help of the TIM22 complex. Carrier precursors emerging from the TOM complex are transferred to small Tim chaperones (Tim9-Tim10 or Tim8-Tim13) (Davis, Alder, Jensen, \& Johnson, 2007; Sirrenberg et al., 1998). After binding of Tim12, the chaperone complex mediates transmission to the TIM22 complex (N. Gebert et al., 2008). In a membrane potential-dependent step the precursor is inserted into the pore and then laterally released into the IM by a yet unknown mechanism (Rehling, Brandner, \& Pfanner, 2004; Rehling et al., 2003). Positively charged residues of the matrix located loops of the protein are assumed to be important for the membrane potential-dependent translocation. Apart from carrier proteins also three TIM subunits with four TMDs use this pathway (Dekker et al., 1997; Dudek et al., 2013). TIM23SORT mediated import: IM proteins that are synthesized as presequencecontaining precursors (preprotein), are recognized by the receptors of the TIM23 $3^{\text {CORE }}$ complex. Once the N-terminus of a preprotein emerges from the TOM complex, it is transferred into the protein-conducting pore of TIM23 ${ }^{\mathrm{CORE}}$, generating a TOM-TIM23 supercomplex (Dudek et al., 2013). Hydrophobic stop-transfer signals downstream of the presequence induce an arrest and lateral release into the IM (Bohnert et al., 2010; Bömer et al., 1997; Glick et al., 1992; van der Laan et al., 2007). For the sorting process TIM23 ${ }^{\mathrm{CORE}}$ associates with Tim21 via Mgr2, leading to the formation of TIM23 ${ }^{\text {SORT }}$. Tim21 recruitment supports the membrane potential-dependent membrane insertion by coupling TIM23 to the respiratory chain (M. Gebert et al., 2012; van der Laan et al., 2006; Wiedemann, van der Laan, Hutu, Rehling, \& Pfanner, 2007). Single spanning IM proteins commonly use the sorting pathway but also few multispanning proteins, like Sym1, are among the substrates (Reinhold et al., 2012). 


\section{INTRODUCTION}

\subsubsection{Soluble proteins of the matrix}

Also soluble proteins of the mitochondrial matrix rely on the TIM23 ${ }^{\mathrm{CORE}}$ complex. In contrast to the mechanism described above, the membrane-potential is not sufficient for full matrix translocation of a protein (Dudek et al., 2013). Instead of Tim21, the presequence translocase-associated import motor (PAM) is recruited to TIM23CORE. By Hsp70-mediated hydrolysis of ATP, the PAM complex provides an additional inward-directed force on the incoming protein (reviewed in detail in Schulz et al., 2015).

\subsection{Mitochondrial protein export}

\subsubsection{Conservative sorting of inner membrane proteins}

A limited number of inner membrane proteins uses a pathway that was first suggested by Hartl and colleagues and comprises re-export of the protein after initial matrix translocation through TIM23 (Hartl, Ostermann, Guiard, \& Neupert, 1987; Hewitt, Gabriel, \& Traven, 2014) (Figure 1-3). Since several aspects seem to be conserved from the bacterial ancestor (Rojo, Stuart, \& Neupert, 1995), this pathway is also called conservative sorting. While conservatively sorted Rip1 depends on the action of Bcs1 (Wagener, Ackermann, Funes, \& Neupert, 2011), membrane insertion of 0xa1 and Cox18 is mediated by the evolutionary conserved Oxa1 translocase (Funes, Nargang, Neupert, \& Herrmann, 2004; Hell, Herrmann, Pratje, Neupert, \& Stuart, 1998; Herrmann, Neupert, \& Stuart, 1997). Based on observations with the multi-spanning protein Mdl1, it was furthermore suggested that lateral release and conservative sorting pathways might work in concert for some proteins (Bohnert et al., 2010). 


\section{INTRODUCTION}

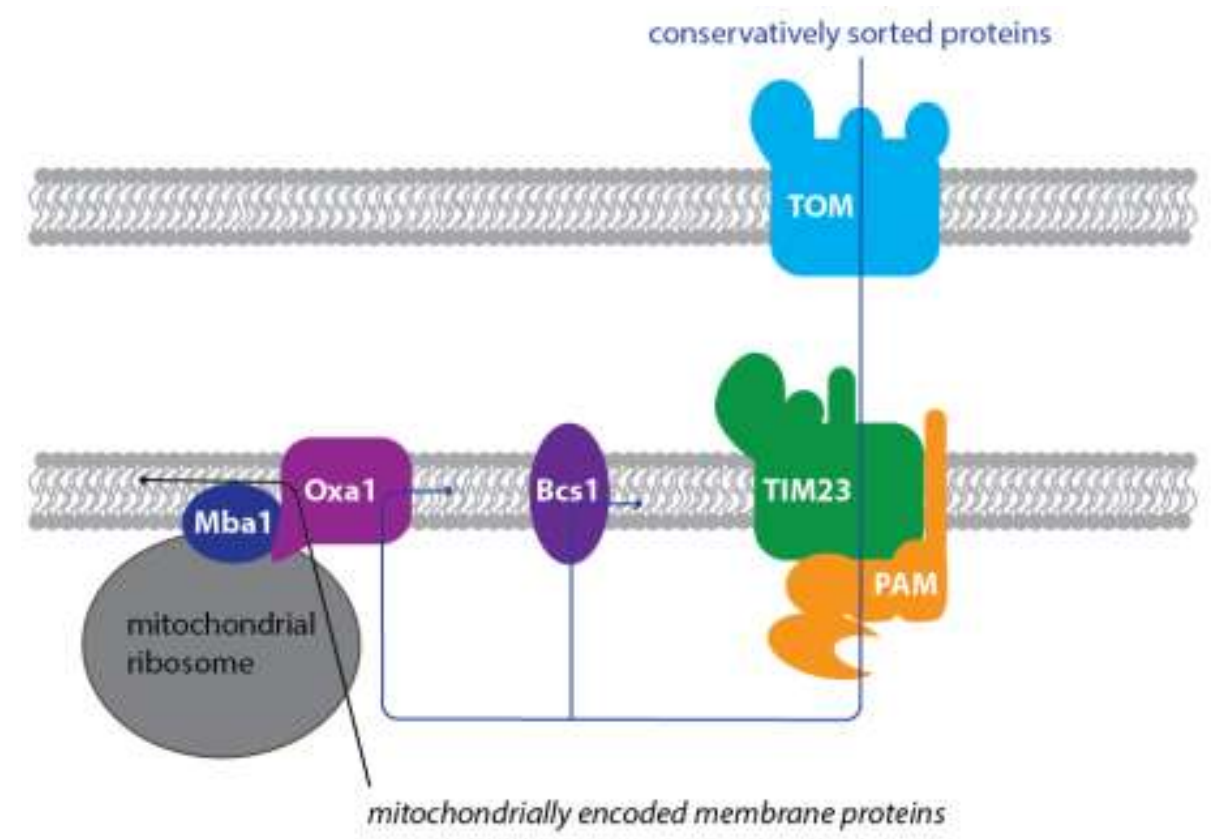

Figure 1-3: Mitochondrial protein export pathways. Conservatively sorted nuclear encoded proteins are fully translocated into the matrix via TOM, TIM23 and PAM. Afterwards, they are inserted into the IM with the help of Oxa1 or Bcs1. Mitochondrially encoded membrane proteins are synthesized on membrane-attached mitochondrial ribosomes and co-translationally inserted via the Oxa1 insertion machinery.

\subsubsection{Membrane insertion of mitochondrially encoded proteins}

In yeast, most proteins encoded by the mitochondrial genome are highly hydrophobic subunits of the respiratory chain located in the IM. To minimize contact with the hydrophilic environment in the matrix, they are translated on membrane-associated ribosomes and co-translationally inserted into the membrane (Ott \& Herrmann, 2010) (Figure 1-3). As in the case of conservatively sorted proteins, insertion is mediated mainly by Oxa1 (Hell, Neupert, \& Stuart, 2001). Oxa1 interacts with mitochondrial ribosomes through its ribosome-binding domain (Jia et al., 2003; Szyrach, Ott, Bonnefoy, Neupert, \& Herrmann, 2003). It furthermore cooperates with the inner membrane proteins Mdm38 and Mba1 (Frazier et al., 2006; Preuss et al., 2001), the latter being proposed to spatially align ribosome and insertion machinery (Pfeffer, Woellhaf, Herrmann, \& Förster, 2015). Oxa1 is responsible for N-terminal export and integration of TMDs of mitochondrial translation products in general. The C-terminus of mitochondrially encoded Cox2 additionally relies on the help of the Oxa1-related protein Cox18 (Saracco \& Fox, 2002). 


\subsection{The oxidative phosphorylation system}

Among the many tasks of mitochondria, their key contribution to energy production is undoubtedly the most famous one. Under respiring conditions, the oxidative phosphorylation (OXPHOS) system, residing within the IM, provides the main energy supply for the eukaryotic cell. Electrons and protons from the degradation of acetyl-CoA in the Krebs cycle are delivered to the respiratory chain in the form of NADH and succinate. The respiratory chain is a series of protein complexes in the IM that transfer electrons from donors to acceptors via redox reactions. Mammalian respiratory chains are composed of four complexes: NADH dehydrogenase (I), succinate dehydrogenase (II), coenzyme Q : cytochrome $c$-oxidoreductase or cytochrome $b c_{1}$ complex (III) and cytochrome $c$ oxidase (IV) (Saraste, 1999) (Figure 1-4 A). In yeast, complex I is substituted by the single proteins Nde1, Nde2 and Ndi1 (Grandier-Vazeille et al., 2001) (Figure 1-4 B). Complex I (or its substitutes) and complex II are the electron-receiving units. Coenzyme $Q$ and cytochrome $c$ shuttle the electrons to complex III and complex IV, respectively. The latter complex is the terminal enzyme of the respiratory chain and catalyzes the reduction of oxygen to water. The electron transfer is coupled to a transfer of protons into the IMS, creating a proton motif force across the IM (Saraste, 1999). This energy is in turn used by the ATP synthase (V) for the phosphorylation of ADP to ATP (reviewed in Yoshida, Muneyuki, \& Hisabori, 2001).

The OXPHOS complexes, except for complex II, contain subunits of dual genetic origin. In yeast, the most hydrophobic subunits of complex III (Cob), complex IV (Cox1, Cox2, Cox3) and complexV (Atp6, Atp8, Atp9) are provided by the mitochondrion itself (Kehrein, Bonnefoy, \& Ott, 2013) (Figure 1-4 B). All other subunits, as well as complex II and the single NADH dehydrogenases, are encoded in the nucleus and follow the import pathways described in sections 1.3 and 1.4. Hence, the spatial and temporal coordination of OXPHOS assembly is of critical importance. A plethora of nuclear encoded protein factors is involved in the regulation of mitochondrial transcription, mRNA maturation, translation, membrane insertion, processing and degradation (Deshpande \& Patel, 2012; T. D. Fox, 2012; Kehrein et al., 2013; Koppen \& Langer, 2007; Mick, Fox, \& Rehling, 2011; Ott \& Herrmann, 2010; Rak, Zeng, Brière, \& Tzagoloff, 2009; Soto, Fontanesi, Liu, \& Barrientos, 2012). Additional assembly factors are essential for the stability and interaction of subunits 


\section{INTRODUCTION}

and assembly modules as well as the insertion of cofactors into the catalytically active subunits of complex III and IV (Mick et al., 2011; Soto et al., 2012; Zara, Conte, \& Trumpower, 2009).

A

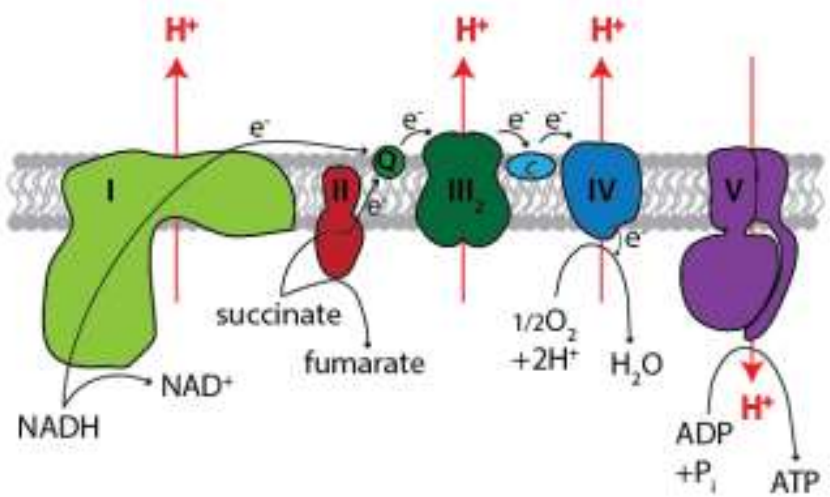

B

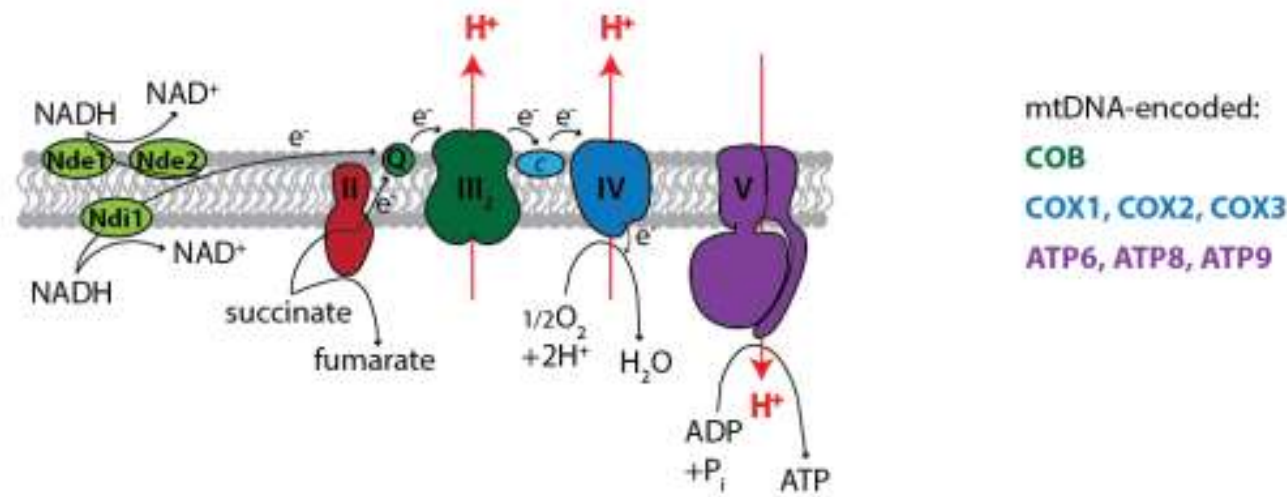

Figure 1-4: Model of the respiratory chain in mammals and yeast. A) The mammalian respiratory chain is composed of electron-receiving units complex I and complex II, small electron carriers coenzyme $Q$ and cytochrome $c$ as well as dimeric complex III and the terminal enzyme complex IV. Apart from transporting electrons, complexes I, III and IV translocate protons across the IM into the IMS, generating an electrochemical gradient. Complex V uses the energy of the gradient to phosphorylate ADP to ATP. B) The yeast oxidative phosphorylation system is organized alike. However, single NADH dehydrogenases Nde1, Nde2 and Ndi1 replace the multimeric complex I of the mammalian system.

\subsubsection{Composition and structure of complex III}

Complex III is an oxidoreductase that uses electrons from the oxidation of coenzmy Q (ubiquinol) for the oxidation of cytochrome $c$. The catalytic core of the enzyme is composed of mitochondrially encoded cytochrome $b$ as well as nuclear encoded cytochrome $c_{1}$ and Rieske iron-sulfur protein (Rip1). It contains three heme groups (in cytochrome $b$ and $c_{1}$ ) and an iron-sulfur cluster (in Rip1). In addition to the evolutionary conserved core, yeast complex III contains seven mitochondria-specific proteins: Cor1, Cor2, Qcr6, Qcr7, Qcr8, Qcr9 and Qcr10 
(Hunte, Koepke, Lange, Rossmanith, \& Michel, 2000; Trumpower, 1990). It is still under debate how these supernumerary subunits contribute to the functionality of mitochondrial complex III. The assembly of the complex is thought to start with the membrane insertion of cytochrome $b$. Guided by complex III-specific assembly factors, cytochrome $b$ runs through a series of assembly intermediates. The enzyme grows with the stepwise incorporation of nuclear encoded subunits (Gruschke et al., 2012; Zara et al., 2009). The assembly is completed upon addition of Qcr10 and Rip1 and the release of remaining assembly factors. In yeast, complex III is organized as a homodimer with each monomer carrying one copy of each subunit, as evident from the crystal structure (Hunte et al., 2000).

\subsubsection{Composition and structure of complex IV}

Complex IV is the terminal enzyme of the respiratory chain that oxidizes cytochrome $c$ to reduce molecular oxygen to water. The evolutionary conserved core is composed of the membrane embedded proteins Cox1, Cox2 and Cox3, which are all encoded in the mitochondrial genome. Cox 1 and Cox 2 contain the cofactors that are needed for catalysis: a dinuclear $\mathrm{Cu}_{\mathrm{A}}$ metal center (in Cox2), a heme $a$ group and a binuclear center composed of heme $a_{3}$ and $\mathrm{Cu}_{\mathrm{B}}$ (in Cox1) (Soto et al., 2012). As for complex III, several supernumerary subunits are imported from the cytosol. In yeast, the mitochondria-specific part of complex IV comprises of Cox4, Cox5a/b, Cox6, Cox7, Cox8, Cox9, Cox12 and Cox13. All of these proteins have mammalian homologues and can be mapped to the respective position in the crystal structure of bovine complex IV (Maréchal, Meunier, Lee, Orengo, \& Rich, 2012; Tsukihara et al., 1996). More recently, further complex IV-associated proteins were described that are not preserved in the crystal structure: Rcf1, Rcf2 and Cox26 (Chen et al., 2012; Levchenko et al., 2016; Strecker et al., 2016; Strogolova, Furness, Robb-McGrath, Garlich, \& Stuart, 2012; Vukotic et al., 2012). The assembly of complex IV is believed to take place in a modular way with an initially independent assembly line for each of the three mitochondrially encoded subunits. Cox1-, Cox2- or Cox3-specific assembly factors mediate proteolytic processing, insertion of cofactors and the interaction with early assembling supernumerary subunits. Once maturation is completed, the Cox 2 and Cox3 modules join the Cox1 assembly intermediate in order to allow for incorporation of late supernumerary subunits (reviewed in Soto 


\section{INTRODUCTION}

et al., 2012). In contrast to the X-ray structure obtained from bovine complex IV, yeast complex IV exists in monomers composed of one copy of Cox1 to Cox12 and probably also Cox26 (Heinemeyer, Braun, Boekema, \& Kouril, 2007; Levchenko et al., 2016; Maréchal et al., 2012). Beyond that, monomers may vary in their composition. Based on recent data, it was suggested that Rcf1, Cox13 and Rcf2 are added in a sequential manner to only a fraction of complex IV, resulting in a specific Rcf/Cox13-containing subpopulation (IV*) (Vukotic et al., 2012). The reasons for the establishment of different versions of complex IV remain elusive.

\subsection{Respiratory supercomplexes}

\subsubsection{Organization of electron transport: solid versus fluid model}

During the first half of the twentieth century, the mitochondrial respiratory chain was discovered and described as the system mediating the redox reactions that account for cellular respiration. Its organization was still unknown. With their proposal of the respiratory chain working as a single entity, Chance and Williams drafted the first solid-state model early on (Chance \& Williams, 1955). Based on several observations, among them the pool behavior of cytochrome $c$ in mammalian mitochondria and the fact that isolated single complexes remain active, the view soon changed towards a random collision (or fluid) model (Hackenbrock, Chazotte, \& Gupte, 1986; Hackenbrock, Schneider, Lemasters, \& Höchli, 1980). This model envisioned single respiratory complexes that are not physically attached to each other, but connected by freely diffusing pools of cytochrome $c$ and coenzyme $Q$ (Figure 1-5 A). Even though yeast cytochrome $c$ did not show pool behavior in most studies, the random collision model stayed the accepted model for more than two decades. It was questioned only when the earlier hypothesized supra molecular assemblies of respiratory complexes were detected biochemically in yeast and mammals by means of blue native (BN) PAGE analysis (Cruciat, Brunner, Baumann, Neupert, \& Stuart, 2000; Schägger \& Pfeiffer, 2000) (Figure 1-5 B). The mammalian supercomplexes identified by Schägger and Pfeiffer contained complexes I, III 2 and IV (in varying amounts), rendering them able to transfer electrons from NADH to oxygen, hence respire. This structure was called respirasome (Schägger \& Pfeiffer, 2000). Due to the lack of multimeric complex I, yeast does not contain true 
respirasomes as found in mammalian mitochondria. The yeast supercomplex structures are built up by complex III dimers interacting with either one or two copies of monomeric complex IV ( $\mathrm{III}_{2} \mathrm{IV}$ and $\mathrm{III}_{2} \mathrm{IV}_{2}$ ) (Cruciat et al., 2000; Schägger \& Pfeiffer, 2000).

A mammals/yeast

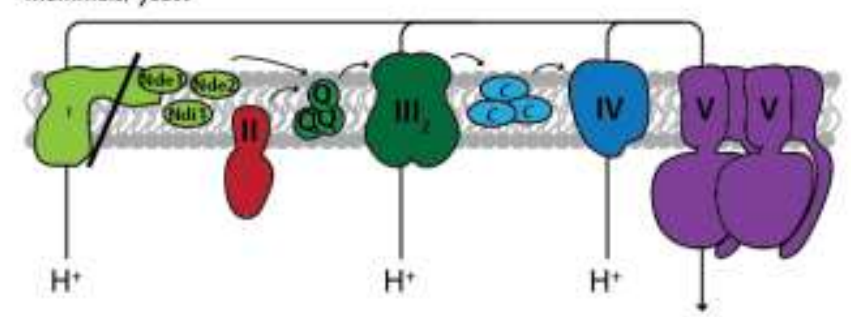

fluid model

B

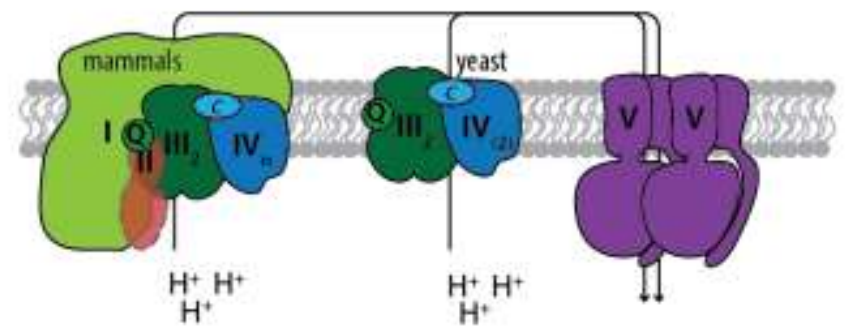

solid model

Figure 1-5: Schematic presentation of fluid and solid view of respiratory chain organization. A) Following the fluid model, each complex of the respiratory chain is an individual entity. Electrons are transferred with the help of diffusing electron carriers (coenzyme Q and cytochrome $c$ ) that are organized in membrane-submersed pools. B) In the solid view, all complexes interact with each other to form large supercomplex structures called respirasomes. In mammals, respirasomes contain complexes I-IV as well as coenzyme Q and cytochrome $c$. Yeast supercomplexes lack complex I as well as complex II. Schematic representations in A) and B) are based on Hackenbrock et al. (1980), Schägger et al. (2001) and Acín-Pérez et al (2014).

Until now, the existence of supercomplex structures of varying compositions has been verified several times and for a plethora of organisms and tissues (reviewed in Lenaz \& Genova, 2012). Acín-Pérez and coworkers succeeded in isolating respirasomes containing all complexes (I-IV) as well as cytochrome $c$ and coenzyme Q (Acin-Perez, Fernandez-Silva, Peleato, Pérez-Martos, \& Enríquez, 2008). This is remarkable, since no other study in yeast or mammals has found complex II attached to other respiratory complexes so far.

\subsubsection{The plasticity model}

There is an ongoing debate between the defenders of the solid and the fluid model. While the solid model cannot accommodate for the pool behavior of mammalian cytochrome $c$, the fluid model fails in the interpretation of BN-PAGE and coisolation studies. However, neither of the models satisfactorily accounts for the sum of kinetic 


\section{INTRODUCTION}

evidence from different species. Acín-Pérez and colleagues observed that metabolically labeled mammalian mitochondrial translation products first assemble into free complexes, followed by supercomplexes after a gap of several hours (AcinPerez et al., 2008). From similar results, Ugalde's group concluded a model that strongly supports the solid state point of view and envisions partially assembled complex I as a scaffold for human supercomplex assembly (Moreno-Lastres et al., 2012). However, this model does not go in line with the observation that single active complex I is detectable in complex IV-deficient mitochondria (Balsa et al., 2012). Therefore, Acín-Pérez and Enriquez proposed a completely new model, which accommodates both old models (Acin-Perez et al., 2008; Acin-Perez \& Enriquez, 2014) (Figure 1-6). The plasticity model considers the respiratory complexes to be a mosaic of individual complexes as well as supercomplex assemblies in varying compositions. Even though the model is based on experimental evidence in mammalian systems, it is also suitable to explain the large amounts of free complex IV usually detected in yeast mitochondrial extracts.

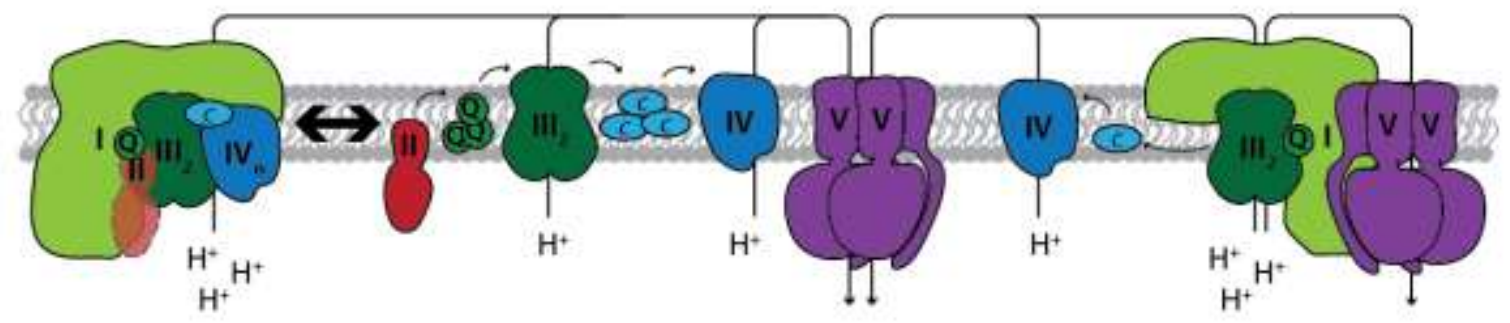

Figure 1-6: Schematic representation of the plasticity model. Following the plasticity model, the respiratory chain exists as a network of individual complexes and supercomplexes with varying composition ( $\mathrm{I}-\mathrm{III}_{2}-\mathrm{IV}_{\mathrm{n}}$ ). The existence of the complex II-containing respirasome as well as the I-III $-\mathrm{V}$ supercomplex is not fully confirmed yet. Modified from Acín-Pérez et al. (2014).

\subsubsection{Physiological reasons for the formation of supercomplexes}

The discovery of respiratory supercomplexes inevitably led to the question for their physiological relevance. In his first review on the topic, Schägger summarized the three possible advantages of supercomplexes over individual complexes that are still most popular (Schägger, 2001): catalytic enhancement, complex stabilization and sequestration of reactive intermediates.

Catalytic enhancement: The close association of complex III with complex IV (in mammals also complexI) dramatically reduces the diffusion distances for the soluble carriers cytochrome $c$ and coenzyme Q. This could lead to faster kinetics. It 


\section{INTRODUCTION}

furthermore enables substrate channeling through short trajectories within the supercomplex structure, accounting for the lack of pool behavior in yeast (Lenaz \& Genova, 2012). However, against this background, the mammalian respiratory chain, displaying pool behavior for cytochrome $c$, would not need supercomplex organization.

Complex stabilization: In mammals, the majority of complex I is bound in either full respirasomes or smaller supercomplexes with complex $\mathrm{III}_{2}$ (Acin-Perez et al., 2008; Schägger, 2001). In the absence of complex III, complex I becomes unstable (AcinPerez et al., 2004; D'Aurelio, Gajewski, Lenaz, \& Manfredi, 2006; Schägger et al., 2004). Even though it was also observed for bacteria (Paracoccus denitrificans), complex I stabilization does not mark a common principle, since it is not conserved in complex I-containing fungi (Lenaz \& Genova, 2012).

Sequestration of reactive intermediates: Panov and colleagues reasoned that the stoichiometry and the channeling of substrates in mammalian respirasomes limits the generation of reactive oxygen species (ROS) (Panov et al., 2007). Since the terminal enzyme, complex IV, is usually found in excess in respirasomes, all upstream units are largely kept oxidized thus preventing the premature reduction of oxygen. In mammals the most critical unit in this respect is complex I, whose ROS production sites might be less exposed in the supercomplex state (Lenaz \& Genova, 2012). In yeast, it rather comprises complex III. Though the idea of limited ROS production due to facilitated electron transfer has been formulated several times (Panov et al., 2007; Schägger, 2001; Seelert et al., 2009), sound experimental evidence is still missing.

\subsubsection{Supercomplex factors: Between assembly and stabilization}

Respiratory supercomplexes are also found in bacteria such as Paracoccus denitrificans. Since bacterial respiratory complexes are exclusively composed of the catalytically active core subunits, it has been speculated that these subunits also mediate supercomplex formation. On the basis of the endosymbiotic theory, mitochondrial supercomplex formation hence might rely on interactions of core subunits. A structural model of the yeast $\mathrm{III}_{2} \mathrm{IV}_{2}$ supercomplex showed close association of conserved complex IV subunits with the surface of complex III and vice versa (Heinemeyer et al., 2007). Nevertheless, formation, maintenance and 


\section{INTRODUCTION}

regulation of mitochondrial, and probably also bacterial, supercomplexes requires more than pure complex core interactions.

Membrane lipids were shown to be important players in supercomplex organization. Cardiolipin proved to be critical for the stabilization of both populations of yeast respiratory supercomplexes $\left(\mathrm{III}_{2} \mathrm{IV}_{2}\right.$ and $\left.\mathrm{III}_{2} \mathrm{IV}\right)$, probably by neutralizing charges of lysine residues in the presumed interaction domains (Bazán et al., 2013; Pfeiffer et al., 2003; Wenz et al., 2009; Zhang, Mileykovskaya, \& Dowhan, 2002; 2005). Whether it moreover supports supercomplex assembly is still discussed controversially (Bazán et al., 2013; Pfeiffer et al., 2003). Apart from cardiolipin, other IM lipids are involved in supercomplex organization. While depletion of cardiolipin leads to a destabilization of supercomplexes, depletion of phosphatidylethanolamine has the opposite effect (Böttinger et al., 2012). Both lipids are crucial for normal enzyme activities (Böttinger et al., 2012) and found in the available crystal structures and structural models of respiratory complexes (Heinemeyer et al., 2007; Mileykovskaya et al., 2012; Tsukihara et al., 1996). In humans, defective biogenesis of cardiolipin causes Barth syndrome, a multi-system disease predominantly linked with cardiomyopathy, emphasizing its physiological relevance (for review see Gaspard \& McMaster, 2015).

In addition, the ADP/ATP carrier (AAC) complex of the IM was reported to influence the stability of supercomplexes in yeast (Dienhart \& Stuart, 2008). At the same time, coupling of AAC to the proton gradient-generating supercomplex renders the energy-demanding ATP transport more efficient. Depletion of cardiolipin does not only lead to a dissociation of supercomplexes but also to a dissociation of ACC from the remaining supercomplexes (Claypool, Oktay, Boontheung, Loo, \& Koehler, 2008). This illustrates the complexity of supercomplex regulation and IM organization in general.

Even though cardiolipin is widely accepted as a mediator for supercomplex formation, researchers had been searching for an additional proteinaceous glue that exceeds the hypothetical catalytic core interactions. This could be envisioned as stabilizing factors or factors that are actually initiating or triggering supercomplex formation. The latter class is of special interest since the composition of supercomplexes seems to be liked to the enzymatic activity which in turn needs to be adapted to the cells demands (Lenaz \& Genova, 2012; Schäfer et al., 2006; 


\section{INTRODUCTION}

Schägger, 2001). The above-mentioned factors would allow for an additional level of supercomplex regulation. Such a regulatory effect has also been proposed for posttranslational phosphorylations on complex I and complex IV, which demonstrably modify enzyme activities (reviewed in Lenaz \& Genova, 2009). In yeast, so far only one protein factor was identified that truly affects inter-complex interactions, and was hence called Respiratory superComplex Factor 1 (Rcf1) (Chen et al., 2012; Strogolova et al., 2012; Vukotic et al., 2012). In its absence, the amounts of $\mathrm{III}_{2} \mathrm{IV}_{2}$ are reduced. However, levels of $\mathrm{III}_{2} \mathrm{IV}$ seem to be unaffected. As illustrated for cardiolipin, it is not clear whether this Rcf1-specific effect is based on a decreased stability or defective assembly of supercomplexes. In the same studies, Rcf1 was described to be a structural subunit of complex IV (Chen et al., 2012; Strogolova et al., 2012; Vukotic et al., 2012). However, a true supercomplex factor would be expected to exclusively associate with supercomplexes but not with individual complexes. Nevertheless, since Rcf1 is present in only a subset of complex IV, it is tempting to speculate that it primes complex IV for supercomplex assembly (Vukotic et al., 2012; Römpler et al., under revision).

Rcf1 has a mammalian homologue, which is expressed in two isoforms: hypoxia-induced HIGD1A (RCF1A) and constitutively expressed HIGD2A (RCF1B). Both isoforms are present in supercomplexes due to their association with complex IV (Chen et al., 2012; Vukotic et al., 2012). However, only RCF1B is able to partially complement for yeast Rcf1 (Vukotic et al., 2012). Chen and colleagues showed a role for RCF1B in the formation of supercomplexes (Chen et al., 2012). In contrast, the RCF1A was proposed to be a regulatory component of complex IV without any impact on supercomplex organization (Hayashi et al., 2015). Closest to a yeast Rcf1-like supercomplex factor seemed to be COX7A2l, as suggested by Enríquez's group (Lapuente-Brun et al., 2013), even though its role in supercomplex formation is highly controversial (Mourier, Matic, Ruzzenente, Larsson, \& Milenkovic, 2014). 


\section{INTRODUCTION}

\subsection{Aims of this study}

The formation of respiration-competent supercomplex structures in mitochondria has been extensively studied in the past decade. On this account, several factors that support respiratory supercomplexes have been identified. Among these are specific protein factors like the yeast protein Rcf1 (Chen et al., 2012; Strogolova et al., 2012; Vukotic et al., 2012). In addition, interactions with lipids (Böttinger et al., 2012; Pfeiffer et al., 2003; Zhang et al., 2005) or with other complexes, like AAC (Dienhart \& Stuart, 2008), have proven to be important. Nonetheless, it still remains to be elucidated whether such interactions simply stabilize an existing supercomplex or whether they are the actual signal for its formation. Following the plasticity model (Acin-Perez et al., 2008), respiratory complexes should be able to constantly change between individual complex and supercomplex state depending on the cells needs. Therefore, a deeper understanding of the regulation of supercomplex formation is needed. In this regard, proteins that are expected to localize to the interface of complex III and IV are of special interest. In yeast, two potential candidates within this category are Rcf1 and Rcf2 (Cui, Conte, Fox, Zara, \& Winge, 2014). Even though initial analysis of Rcf2, unlike Rcf1, found that this protein is not essential for supercomplex formation (Vukotic et al., 2012), it has uncovered additional properties worthy of further investigation. Rcf2 is partly processed upon import into mitochondria, rendering it interesting in terms of possible regulatory functions. Along these lines, the investigation of the nature and the timing of this processing step, as well as its impact on mitochondrial functions, was one of the main aims of this study.

In addition, in silico analysis and alignments revealed a faint but statistically significant similarity between Rcf1, Rcf2 and a third, so far uncharacterized, protein encoded by the gene YBR255C-A. These alignments demonstrate that the two fragments originating from the above mentioned Rcf2 processing would resemble Rcf1 and YBR255C-A respectively. Therefore, the second part of this study deals with a basic characterization of YBR255C-A and addresses a possible interaction with respiratory (super) complexes.

Finally, the postulated Rcf protein family is analyzed in greater detail for a potential interplay and functional redundancy with regard to respiratory function. 


\section{Results}

\subsection{Maturation and assembly of the complex IV subunit Rcf2}

In initial analyses of Rcf2, the protein was described as a substoichiometric subunit of complex IV that is not essential for respiration or supercomplex formation under the tested conditions (Strogolova et al., 2012; Vukotic et al., 2012). For the published investigations, in vitro synthesized radiolabeled Rcf2 was imported into isolated mitochondria. As the main focus lay on its assembly into supercomplexes, a surprising effect was largely overlooked, namely the emergence of a shortened version of Rcf2 (Figure 2-1). Based on this finding, a second, more in-depth characterization of Rcf2 was initiated, predominantly focusing on a potential processing of the imported protein.

\subsubsection{Identification of the Rcf 2 processing site}

Rcf2 precursors, radiolabelled with [35S]-methionine, translocate into isolated wildtype mitochondria in a partially membrane potential-independent manner, as previously published (Vukotic et al., 2012). Upon import, a fraction of the protein is processed into a smaller fragment of about $21 \mathrm{kDa}$. The signal of the fragment was weak, but still detectable, after treatment with proteinase $\mathrm{K}$ and was absent from the input (Figure 2-1A). When mitochondrial lysates were tested with an antibody against the Rcf2 C-terminus, an Rcf2-specific signal was detected at the size of the radiolabeled fragment. The size of the cleaved sequence and the fact that only a small fraction of the protein is processed argue against this processing being a classical presequence removal. Such a presequence has also not been predicted for Rcf2 (Vukotic et al., 2012). It was therefore concluded that the fragment generated after import represents an N-terminally truncated additional version of Rcf2 $\left(\operatorname{Rcf} 2^{C}\right)$. To define the processing site, a set of radiolabeled N-terminally truncated Rcf2 constructs was designed (Figure 2-1B), synthesized in vitro and compared to the endogenous $\operatorname{Rcf}^{C}$ signal detected by the antibody (Figure 2-1C). This experiment revealed an unexpectedly high sensitivity of the Rcf2 antibody. It was able to detect minor amounts of in vitro synthesized protein. Specificity was ensured by a comparison of $r c f 2 \Delta$ with construct $f$ (aa1-208), which lacks the C-terminal antibody 


\section{RESULTS}

epitope but gives a strong signal in the autoradiogram. Construct $c 1$ (aa62-224) proved to be the closest to the endogenous $\operatorname{Rcf} 2^{C}$, which narrows the processing site down to a region around amino acid 62. Following the predicted topology of Rcf2, depicted in Figure 2-1D, the site should be located within TMD2.
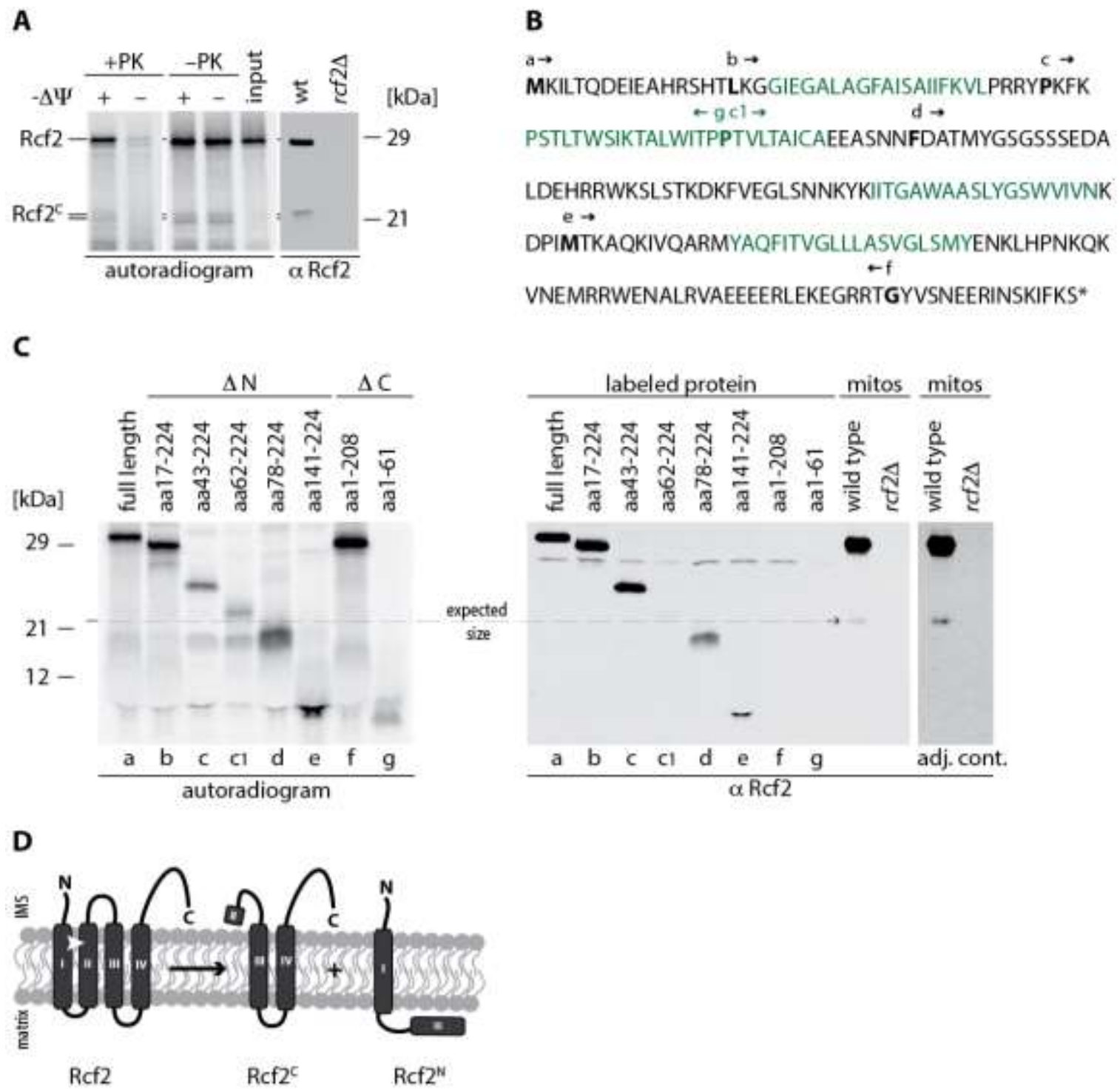

Figure 2-1: A fraction of Rcf2 is processed upon import into mitochondria. A) Radiolabeled Rcf2 was imported into isolated mitochondria for $15 \mathrm{~min}$ in the presence or absence of membrane potential $(\Delta \Psi)$. Samples were treated with proteinase $\mathrm{K}(\mathrm{PK})$, where indicated and analyzed by SDS-PAGE and digital autoradiography. For comparison with endogenous Rcf2 ${ }^{\mathrm{C}}$, mitochondria were analyzed by SDS-PAGE and western blotting. B) Amino acid sequence of Rcf2 with TMDs indicated in green. Bold characters mark start or stop positions for the truncations. C) Non-imported radiolabeled $\mathrm{N}$ - and C-terminally truncated Rcf2 constructs were analyzed by SDS-PAGE and compared to endogenous $\mathrm{Rcf} 2^{\mathrm{C}}$ as in A. Construct $a$ represents full length Rcf2. D) Predicted model of the Rcf2 processing event.

The corresponding N-terminal fragment, $\operatorname{Rcf} 2^{\mathrm{N}}$, is represented by construct $g$ (aa1-61). It has to be noted, that endogenous $\operatorname{Rcf}^{\mathrm{N}}$ does not contain methionine and 
was therefore not observed in the initial import experiment. For visualization, several methionine residues were added to the C-terminus of construct $g$. Endogenous $\operatorname{Rcf} 2^{\mathrm{N}}$ is not immunodetectable either, since it lacks the C-terminal antibody epitope.

To enable detection of Rcf2 ${ }^{\mathrm{N}}$, an N-terminally FLAG-tagged Rcf2 construct (FLAGRcf2) was generated. Given that both parts of the protein are stable, processing of this construct should result in untagged Rcf2 ${ }^{\mathrm{C}}$ and FLAG-tagged Rcf2 ${ }^{\mathrm{N}}$ (FLAGRcf2 ${ }^{\mathrm{N}}$ ) that is detectable by an antibody directed against the FLAG tag (Figure 2-2A).

A

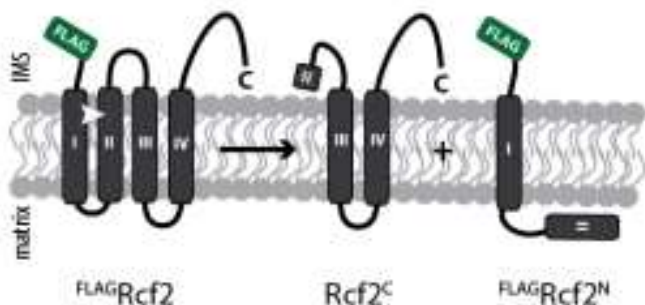

FLAGRCf2

$\operatorname{Rcf} 2^{C}$
B

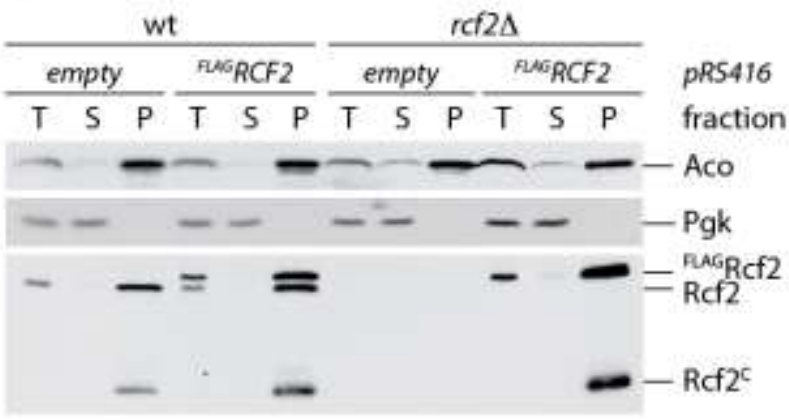

C

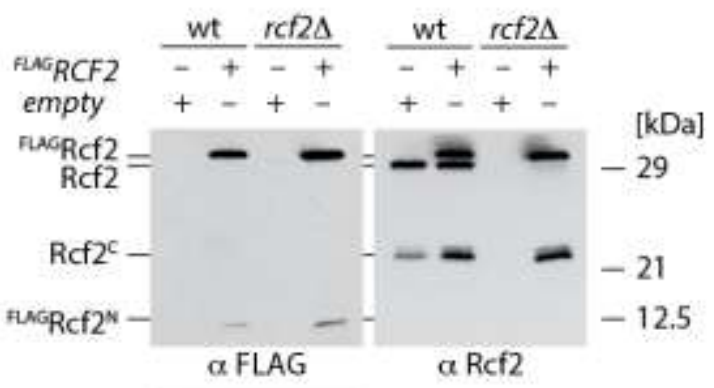

Tom70 - - - -

Figure 2-2: FLAGRcf2 localizes to mitochondria and enables detection of Rcf2 ${ }^{\mathrm{N}}$ and Rcf2C. A) Model visualizing the position of the FLAG tag in Rcf2 and Rcf2 ${ }^{\mathrm{N}}$. B) Wild-type (wt) and $r c f 2 \Delta$ cells expressing plasmid-born ${ }^{\text {FLAGRcf2 }}$ were subjected to subcellular fractionation by differential centrifugation. Samples of homogenized cells (T), cytosolic supernatant (S) and organellar pellet (P) were analyzed by SDS-PAGE and western blotting. Aco and Pgk1 were used as mitochondrial and

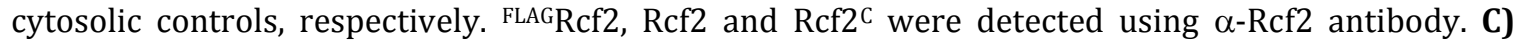
Isolated mitochondria of the strains used in D were analyzed by SDS-PAGE and western blotting. Rcf2 variants were detected using $\alpha$-FLAG ( ${ }^{\mathrm{FLAG}} \mathrm{Rcf} 2$ and ${ }^{\mathrm{FLAG}} \mathrm{Rcf} 2^{\mathrm{N}}$ ) and $\alpha$-Rcf2 antibodies ( ${ }^{\mathrm{FLAG} R c f 2, \mathrm{Rcf} 2}$ and $\mathrm{Rcf}^{\mathrm{C}}$ ). Tom70 served as a loading control.

FLAGRcf2, expressed from a centromeric plasmid in wild-type and $r c f 2 \Delta$, correctly localized to mitochondria as shown by subcellular fractionation in Figure 2-2B. Like endogenous Rcf2, FLAGRcf2 was detected in total and organellar fractions, but not in the cytosolic supernatant. The cytosolic control protein, Pgk1, was exclusively present in the supernatant. $\operatorname{Rcf}^{\mathrm{C}}$ is too low in abundance to be detected in the total, 
but was nicely enriched in the organellar fractions and absent in the cytosolic supernatant. Hence, the N-terminal tag does not interfere with correct Rcf2 processing. Despite usage of the endogenous Rcf2 promoter, expression level of FLAGRcf2 slightly exceeded the level of endogenous protein, as assessed by detection of both proteins with the $\alpha$-Rcf2 antibody. Analysis of isolated mitochondria from FLAGRcf2-expressing strains with the $\alpha$-FLAG antibody confirmed the existence of FLAGRcf2 ${ }^{N}$ (Figure 2-2C). The observed signal is specific for FLAGRcf2-expressing strains and appeared at the expected size of about $12 \mathrm{kDa}$.

\subsubsection{Refinement of the Rcf2 topology model}

Based on the model depicted in Figure 2-1D, the processing site is situated within a predicted transmembrane region of Rcf2, leaving a rather limited set of potential proteases that could be responsible for processing. However, this model is based on a predicted topology that has not yet been verified. Prior to an extensive search for the protease, it was deemed appropriate to confirm the number and orientation of transmembrane domains (TMDs). From data obtained by protease protection assays during the initial analysis, an IMS localization of the C-terminus had already been postulated (Vukotic et al., 2012). To test whether the same holds true for the N-terminus, mitochondria isolated from a genomic RCF2 knockout expressing FLAGRcf2 were subjected to the same analysis. When intact mitochondria are exposed to proteinase $\mathrm{K}$, all proteins protected by the outer mitochondrial membrane should remain stable. Figure 2-3A shows a slight destabilization of control proteins of the inner mitochondrial membrane (Tim21 and Mic10) and also FLAGRcf2. This indicates that the mitochondrial isolation procedure may have caused slight damage to the mitochondrial network. Nevertheless, a further reduction of Tim21 and Mic10 was observed in proteinase K-treated mitoplasts, while the inner membrane-protected matrix protein, Tim44, remained stable. FLAGRcf2, detected with the $\alpha$-FLAG antibody, behaved like Tim21 and Mic10, indicating that its N-terminal antibody epitope is protease-accessible and hence is also located in the IMS (Figure 2-3 A+B). 
A

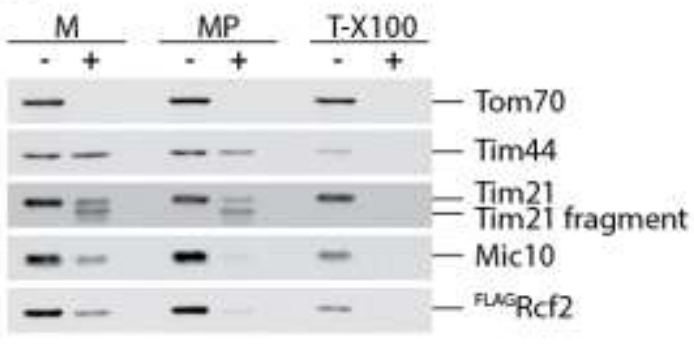

c

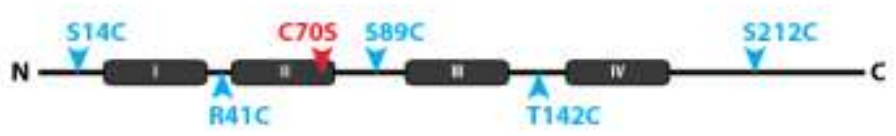

$\mathbf{E}$

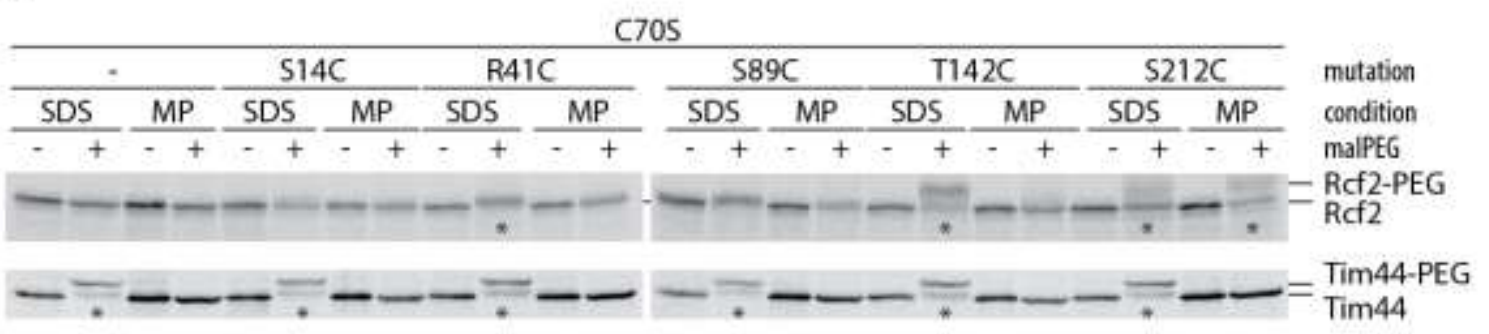

Figure 2-3: Rcf2 exhibits a four TMD conformation with its $\mathrm{N}$ - and C-terminus facing the IMS. A) Wild-type mitochondria were left untreated (M), swollen (MP) or lysed with 1\% Triton X-100 (T X-100), treated with Proteinase $\mathrm{K}$ where indicated and subjected to SDS-PAGE and western blotting. B) Model showing IMS localization of the Rcf2 N-terminus. C+D) Models indicating the positions of the removed/introduced cysteine residues in the amino acid sequence as well as in the predicted topology. E) After import of radiolabeled Rcf2, mitochondria were treated with proteinase $\mathrm{K}$ followed by either swelling (MP) or lysis in SDS (SDS). Samples were incubated in $\mathrm{CuSO}_{4}$ and subsequently analyzed by SDS-PAGE and autoradiography, followed by immunodetection of Tim44.

With its $\mathrm{N}$ - and C-termini facing the IMS, the protein should contain either two or four TMDs. In the original publication, Rcf2 was described as a protein containing two TMDs (Vukotic et al., 2012). In contrast, TMpred, an algorithm based on the statistical analysis of a database of naturally occurring transmembrane proteins (Hofmann \& Stoffel, 1993), strongly prefers a model with four TMDs. To distinguish between these two possibilities, cysteines were introduced into Rcf2 and treated with maleimide PEG to determine their localization relative to the inner mitochondrial membrane. Maleimide PEG covalently binds to sulfur exposed by cysteine residues, thus adding a specific mass $(2 \mathrm{kDa})$ to the modified protein. This 
compound, however, cannot cross biomembranes such as the inner mitochondrial membrane. In mitoplasts, only cysteines facing the IMS are accessible for modification, while in SDS-lysed mitochondria, every cysteine should be able to react. A comparison of the modification pattern in mitoplasts and lysed mitochondria enables the discrimination between IMS and matrix cysteines. The mutant versions of Rcf2 used for the experiment were all based on the cysteine-free Rcf2 ${ }^{270}$. Although the endogenous cysteine proved to be inaccessible to maleimide PEG in preliminary experiments, it was removed to fully exclude interferences. The additional cysteines where then introduced into the predicted inter-helix loops in the IMS and the matrix, as well as into the N- and the C-termini (Figure 2-3 C and D). Radiolabeled in vitro synthesized mutant versions of Rcf2 were imported into isolated mitochondria, which were subsequently lysed or used to generate mitoplasts, prior to treatment with maleimide PEG. Tim44 served as an intrinsic control and proof of principle for the experimental setup. This protein contains one cysteine, which is protected by the inner mitochondrial membrane in mitoplasts and only modified in lysed mitochondria. Figure 2-3 E shows the respective expected modification pattern. A similar behavior of C41 and C142 in the Rcf2 mutants confirmed these to have positions within the matrix, thereby confirming the first and fourth TMD. Unfortunately, two of the introduced cysteines were not modifiable in general (Figure 2-3 E - S14C and S89C). Among these was the most interesting cysteine at position 89. C89 is close to the endogenous cysteine C70, which could not be modified either. While C70 most likely resides within TMD2, which could hinder modification, C89 should be well away from the membrane. It was placed in the middle of the loop between TMD2 and TMD3 (Figure 2-3). It is therefore questionable whether it is in general possible to find a position accessible for maleimide PEG in this loop. For this reason, these experiments were not followed up. Nevertheless, the hydrophobicity plot used for the topology prediction showed a rather strong hydrophobic profile for TMD3 (data not shown). Despite incomplete experimental evidence, these results point towards a four TMD model and this was therefore adopted for the following analyses. 


\subsubsection{The role of intramembrane proteases in Rcf2 processing}

As mentioned before, processing within a TMD limits the number of proteases that could potentially be responsible for cleavage. In general, the helical conformation of a TMD makes it a rather poor protease substrate (Hubbard, 1998; Tyndall, Nall, \& Fairlie, 2005). For this reason, substrates of several intramembrane proteases have been shown to contain helix-destabilizing residues, which might promote local helix unfolding prior to proteolysis (Akiyama \& Maegawa, 2007; Urban \& Freeman, 2003; Ye, Davé, Grishin, Goldstein, \& Brown, 2000). Consistently, the second TMD of Rcf2 contains two prolines in the vicinity of the approximated cleavage site, as well as several alanines, rendering it a suitable substrate. Furthermore, this region meets the requirements for a recognition motif identified for rhomboid proteases (rhomboids), one of the currently best-understood families of intramembrane proteases. These requirements are: a small residue in position P1, along with hydrophobic and large residues in P4 and P2' (Strisovsky, Sharpe, \& Freeman, 2009). Strikovsky and colleagues showed the functional importance of this motif for the cleavage of different substrates (e.g. Drosophila Spitz and bacterial TatA) by a diverse set of rhomboids. The yeast mitochondrial rhomboid, Pcp1, is a serine protease that is responsible for cleavage of the dynamin-like GTPase Mgm1, generating a short isoform of Mgm1 that is important for the mitochondrial fusion machinery (Herlan, Vogel, Bornhovd, Neupert, \& Reichert, 2003; McQuibban, Saurya, \& Freeman, 2003; Sesaki, Southard, Hobbs, \& Jensen, 2003). To test whether Pcp1 is also responsible for processing of Rcf2, mitochondria isolated from a genomic knockout for PCP1 were analyzed for levels of Rcf2 and Rcf2C (Figure 2-4 A). pcp1 $\triangle$ showed reduced amounts of several tested proteins, including Rcf2. When compared to full length Rcf2, the decrease in $\operatorname{Rcf} 2^{C}$ seemed to be slightly more prominent. Nevertheless, processing in general is still taking place in the absence of Pcp1. Despite the striking similarity of the Rcf2 processing site with known rhomboid substrates, Pcp1 does not seem to be the major processing enzyme of Rcf2.

To screen for an alternative protease, the next step was to define the protease class. Depending on their proteolytic mechanism, proteases can be specifically inhibited by different substances. EDTA inhibits metalloproteases by chelating bivalent metal ions, while pepstatin A specifically inhibits aspartyl peptidases (Umezawa, Aoyagi, Morishima, Matsuzaki, \& Hamada, 1970). Complete ${ }^{\mathrm{TM}}$ (Roche) is an inhibitor cocktail 


\section{RESULTS}

directed against serine and cysteine proteases. To test whether one of these inhibitors prevents cleavage of Rcf2, radiolabeled Rcf2 or Cox13 were imported into isolated mitochondria pretreated with either individual or mixed inhibitors. The maturation of Cox13 by the metalloprotease MPP was monitored as an intrinsic control for the efficiency of the inhibitor pretreatment. Indeed, the signal for mature Cox13 was absent in samples treated with the chelating agent EDTA (Figure 2-4 B; lanes 4, 6 and 7). In contrast, $\operatorname{Rcf}^{2}{ }^{C}$ was always detected, also in a sample treated with inhibitors against all known classes of proteases (Figure 2-4 B; lane 7).

A

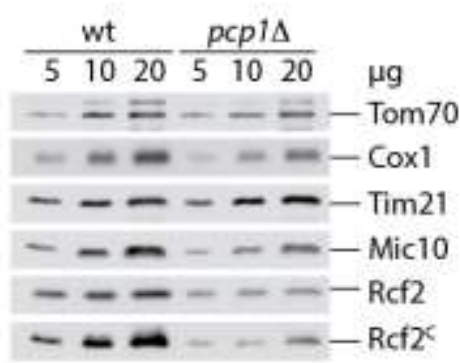

B

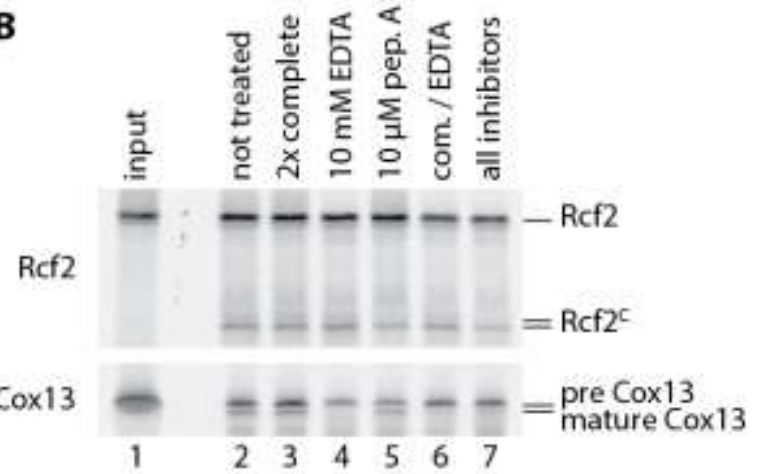

Figure 2-4: Deletion of PCP1 and treatment with common protease inhibitors do not affect the processing of Rcf2. A) Isolated mitochondria of wild-type (wt) and $p c p 1 \Delta$ were analyzed by SDS-PAGE and western blotting. B) Radiolabeled Rcf2 or Cox13 (lane 1) were imported into isolated wild-type mitochondria pretreated with the indicated inhibitors (lanes 2-7). Samples were subjected to SDS-PAGE and autoradiography. For separation of pre and mature Cox13, the gel was supplemented with urea.

\subsection{4. $\operatorname{Rcf}^{\mathrm{C}}$ is associated with complex IV while $\operatorname{Rcf}^{\mathrm{N}}$ is not}

Even though the protease could not be identified, the analysis so far has clearly shown the presence of two shortened versions of Rcf2, in addition to the full length protein, in mitochondria. The next step was to address the fate of both fragments, focusing especially on a potential complex IV and/or supercomplex association of $\operatorname{Rcf2}^{\mathrm{C}}$ and $\mathrm{Rcf}^{\mathrm{N}}$. In a two-dimensional gel analysis of isolated mitochondria, the endogenous full length protein usually segregates into three different pools (Figure 2-5 A). In the first and second pool it comigrates with supercomplexes and monomeric complex IV respectively. The predominant version of complex IV at steady state is slightly smaller than the main Rcf2-containing version of complex IV. Nevertheless, the latter still contains Cox1 (and other structural subunits) and is indicated as complex IV*. The third pool migrates at roughly $100 \mathrm{kDa}$ and cannot be 
assigned to a specific protein complex. $\mathrm{Rcf}^{\mathrm{C}}$ comigrated with the first and second pool, but not with the third (Figure 2-5 A). Thus it seems to be a component of complex IV*. To confirm the interaction predicted from the comigration pattern, complex III and IV were isolated and the elution fractions were tested for the presence of Rcf2C (Figure 2-5 B). To maintain a preferably natural protein environment, the experiment was first carried out in tag-free strains, using specific antibodies directed against structural subunits of complex IV (Cox2) and III (Qcr8). Both antibodies were able to isolate detectable amounts of bait from digitioninsolubilized mitochondria and coisolated both, full length and truncated Rcf2. However, compared to the levels of complex III/ IV and Rcf2 ${ }^{C}$ that were coisolated with Rcf2, the isolation efficiency in general was rather poor.

A

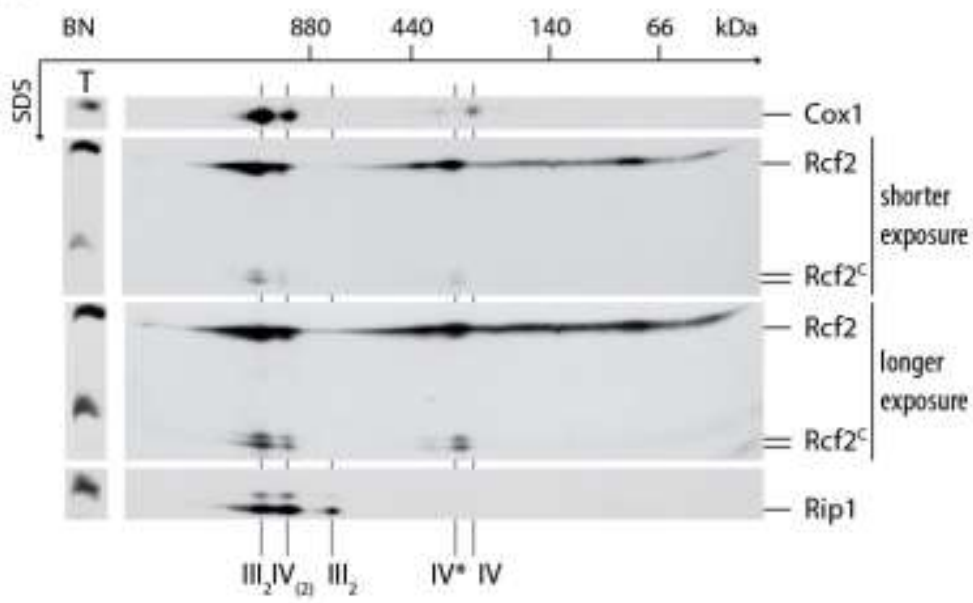

B

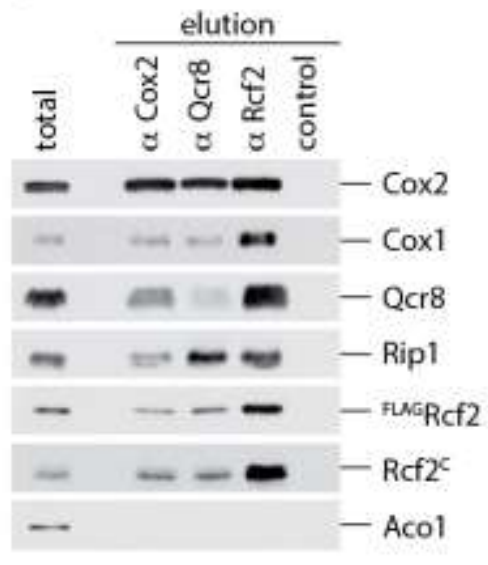

Figure 2-5: Rcf2 ${ }^{C}$ comigrates with and is coisolated by complex IV and respiratory supercomplexes. A) Isolated wild-type mitochondria were solubilized in 1\% digitonin and analyzed by BN-PAGE followed by a second dimension SDS-PAGE and western blotting. Cox1 and Rip1 were used to mark the positions of respiratory supercomplexes $\left(\mathrm{III}_{2} \mathrm{IV}_{2}\right.$ and $\left.\mathrm{III}_{2} \mathrm{IV}\right)$, complex $\mathrm{III}_{2}$ and complexes IV / IV* (total: $10 \mu \mathrm{g}$ ). B) Digitonin-solubilized wild-type mitochondria were used for immunoprecipitation of Cox2, Qcr8 and Rcf2. Pam18 served as a negative control. Total (6\%) and eluates $(100 \%)$ were analyzed by SDS-PAGE and western blotting.

The isolation of ZZ-tagged Cox4 on the other hand enabled efficient isolation of complex IV and supercomplexes (Figure 2-6 A), as evidenced by the amount of Cox1 and Rip1 present in the elution. Despite enrichment of full length Rcf2 in TEV-cleaved Cox4 ${ }^{\mathrm{ZZ}}$ eluates, only minor amounts of $\mathrm{Rcf2}{ }^{\mathrm{C}}$ were detectable in the SDS-PAGE. Two-dimensional analysis of the eluate revealed the presence of Rcf2C in the Rcf2-containing complex IV* population that was coisolated with the structural subunit Cox4 (Figure 2-6 B). 


\section{RESULTS}

A

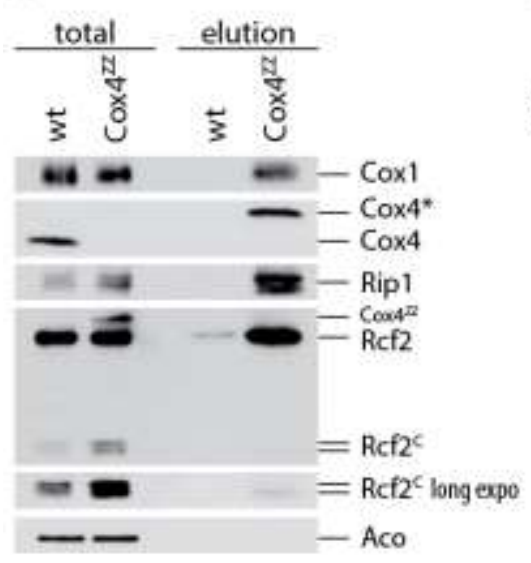

B

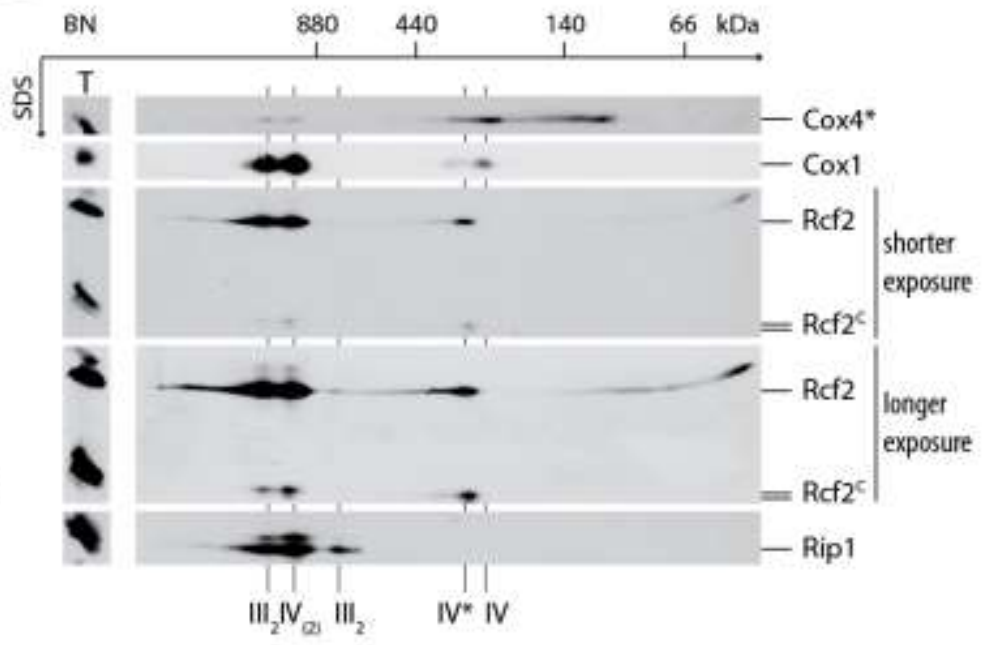

Figure 2-6: Rcf2C is associated with complex IV*, a specific population of complex IV. A) Digitonin-solubilized mitochondria isolated from wild-type (wt) and Cox $4^{\mathrm{ZZ}}$ strains were subjected to IgG chromatography. Upon TEV protease cleavage, elutions were analyzed by SDS-PAGE and western blotting (total: 5\%; elution: 100\%). B) TEV-cleaved Cox4ZZ eluate from A was used for BN-PAGE followed by a second dimension SDS-PAGE and western blotting. Cox1 and Rip1 were used to mark the positions of respiratory supercomplexes $\left(\mathrm{III}_{2} \mathrm{IV}_{2}\right.$ and $\left.\mathrm{III}_{2} \mathrm{IV}\right)$, complex $\mathrm{III}_{2}$ and complexes IV / IV*. Cox4* marks Cox4 after removal of ZZ by TEV cleavage (total: $10 \mu \mathrm{g}$ ).

To verify association of $\operatorname{Rcf}^{c}$ with complex IV components, this analysis was performed reciprocally. Rcf2-containing complexes were isolated via FLAGRcf2 in the genomic $r c f 2 \Delta$ background. As depicted in Figure 2-7 A, the isolation via the $\mathrm{N}$-terminal FLAG tag was extremely efficient and strongly copurified complex IV (Cox1) and III (Qcr8). Total, elution and unbound samples were then compared in a two-dimensional PAGE analysis. Figure 2-7 B demonstrates that FLAGRcf2 specifically copurified complex IV*, as indicated by the enrichment and comigration of Cox1 and Cox2 with FLAGRcf2 and Rcf2C. Based on the migration pattern of Cox1 and Cox2 in total and elution samples, the predominant pool of monomeric complex IV did not contain FLAGRcf2, which therefore remained in the unbound fraction. Remarkably, even though $\operatorname{Rcf}^{C}$ was coisolated along with FLAGRcf2, a considerable amount that associated with complex IV or supercomplexes remained in the unbound. Even though isolation via the FLAG tag did not lead to considerable enrichment of the $100 \mathrm{kDa}$ pool of Rcf2, it was absent in the unbound sample. At the same time, a strong signal for full length Rcf2 appeared in a range below $66 \mathrm{kDa}$ in the eluate. This could hint towards a dissociation of the protein from different complexes during the course of the isolation. 
A

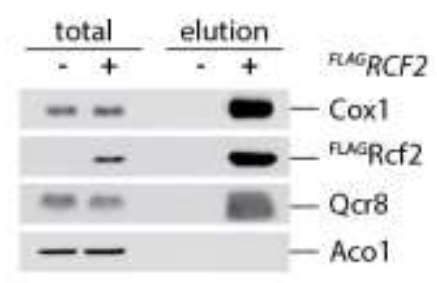

B

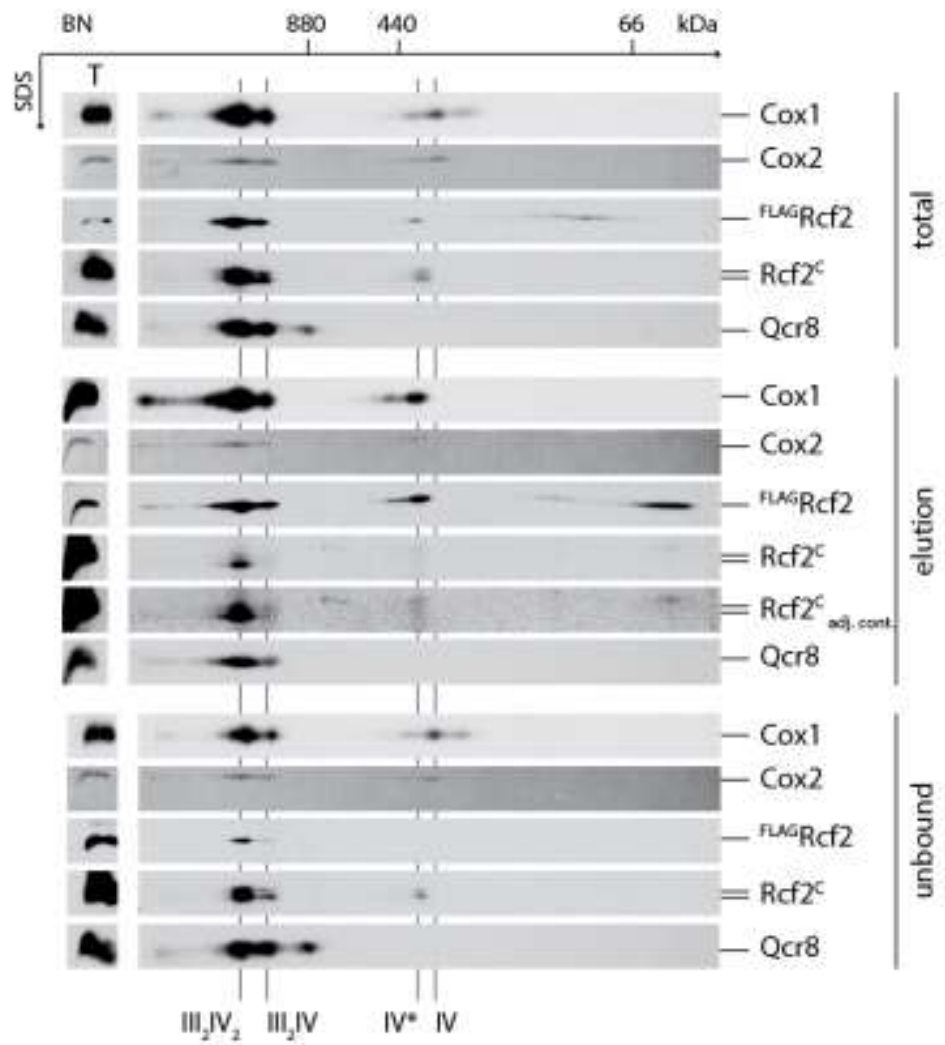

Figure 2-7: Complex IV* is specifically enriched by isolation of Rcf2. A) Digitonin solubilized $r c f 2 \Delta$ / FLAGRcf2 mitochondria were used for immunoprecipitation via FLAG. Samples were eluted with FLAG peptide. Total (3\%) and elution (100\%) were subjected to SDS-PAGE and western blotting. $r c f 2 \Delta(-)$ was included as a control. B) Total, elution and unbound from A were subjected to BN-PAGE followed by a second dimension SDS-PAGE and probed for markers for respiratory complexes (Cox1,

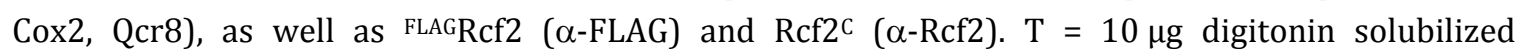
mitochondria.

Figure 2-7 B also demonstrates that the N-terminal FLAG tag does not interfere with the protein's association with complex IV*. It could therefore be used as a suitable tool for the analysis of Rcf2 ${ }^{\mathrm{N}}$ migration patterns. Mitochondria isolated from FLAGRcf2-expressing $r c f 2 \Delta$ were subjected to two-dimensional PAGE, and probed

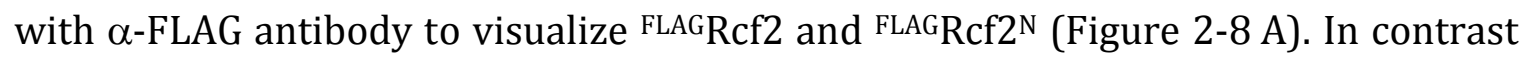
to Rcf2C, FLAGRcf2N did not comigrate with the full length protein in any of the three Rcf2 pools. Using a long exposure, a faint signal with a peak in the range below $66 \mathrm{kDa}$ was detected and suspected to be free FLAGRcf2 ${ }^{\mathrm{N}}$. Generally, the detection of digitonin-solubilized FLAGRcf2 ${ }^{\mathrm{N}}$ is extremely poor, while mitochondrial lysates generated in regular SDS sample buffer allow for a clear, albeit weak, FLAG signal. To test whether this effect is based on the detergent or rather on an overall instability of Rcf2 ${ }^{N}$, the solubilization properties of ${ }^{F L A G} R c f 2, \operatorname{Rcf} 2^{C}$ and ${ }^{F L A G} R c f 2{ }^{N}$ were analyzed in different detergents (Figure 2-8 B). Strikingly, none of the tested 


\section{RESULTS}

detergents were able to preserve ${ }^{\text {FLAGRcf }} 2^{\mathrm{N}}$, while ${ }^{\text {FLAGRcf2 }}$ and $\operatorname{Rcf}^{\mathrm{C}}$ were always visible, although to different extents. FLAGRcf2 ${ }^{\mathrm{N}}$ was exclusively detectable in mitochondria lysed in SDS sample buffer. Since isolation via the FLAG tag did not result in a noticeable enrichment of FLAGRcf2 ${ }^{\mathrm{N}}$ either (data not shown), it can be assumed that this fragment is unstable.

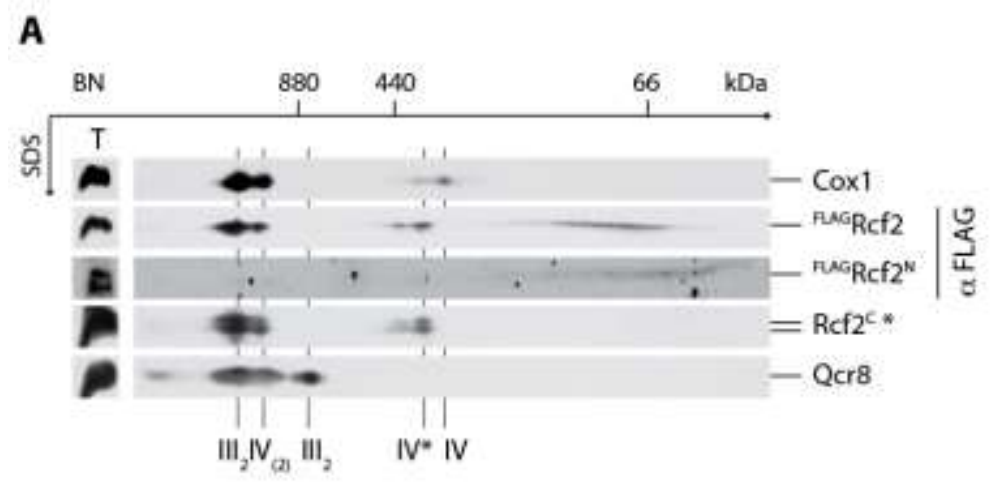

B

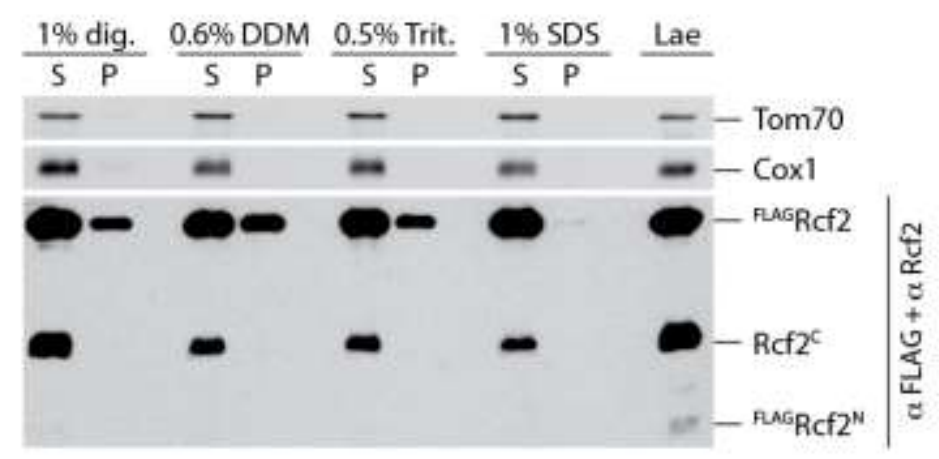

Figure 2-8: FLAG Rcf2 $^{\mathrm{N}}$ is highly unstable and does not associate with any of the Rcf2-containing complexes. A) Isolated mitochondria of FLAGRcf2-expressing $r c f 2 \Delta$ were solubilized in $1 \%$ digitonin and analyzed by BN-PAGE followed by second dimension SDS-PAGE and western blotting. Cox1 and Qcr8 were used to mark the positions of respiratory supercomplexes ( $\mathrm{III}_{2} \mathrm{IV}_{2}$ and III $\mathrm{IV}$ ), complex $\mathrm{III}_{2}$ and complexes IV / IV*. Rcf2 ${ }^{\mathrm{C}}$ was detected with $\alpha$-Rcf2 antibody $\left(^{*}\right)$. T = $10 \mu \mathrm{g}$ digitonin solubilized mitochondria. B) The same mitochondria as in A were solubilized using the indicated detergent or resuspended in SDS sample buffer (Lae). Samples were analyzed by SDS-PAGE and western blotting.

\subsubsection{Assembly of Rcf2 into supercomplexes depends on its C-terminus}

Since the protease could not yet be determined, it is unclear where and when the processing of Rcf2 takes place. Assuming a post-import processing step, further dissection is required to determine whether cleavage occurs prior to, during, or after assembly into complex IV. In the case of co- or post-assembly processing, pretruncated Rcf2 might be unable to assemble into supercomplexes. To test this hypothesis, truncated Rcf2 constructs were used for in vitro import and assembly studies. All truncations were able to translocate into a protease-protected 
mitochondrial compartment in a partially membrane potential-independent manner, as observed for the full length protein (Figure 2-9 lower panel). Furthermore, the Rcf2C-resembling construct, $C_{1}$ (aa63-224), was assembled into supercomplexes, even more efficiently than full length Rcf2 (Figure 2-9 upper panel). In contrast, the Rcf2 ${ }^{\mathrm{N}}$-ressembling construct, $G$ (aa1-62), was unable to assemble into any high molecular weight complexes. Likewise, every other C-terminal truncation, with the exception of construct $F$ (aa1-208), failed to reach supercomplexes and did not assemble into any smaller complexes either. Construct $F$ (aa1-208) is only shortened by the last 16 amino acids. Therefore, it still contains the main portion of the IMS domain following the last TMD. Unlike the other C-terminal truncations, it was efficiently integrated into supercomplexes. Hence, the assembly of Rcf2 depends on the presence of TMD3, TMD4 and at least a part of the C-terminal IMS domain, which roughly corresponds to Rcf2c. This strongly hints to a pre-assembly processing after which only $\operatorname{Rcf2}^{C}$ is further assembled into complex IV, as is the unprocessed protein. 


\section{RESULTS}

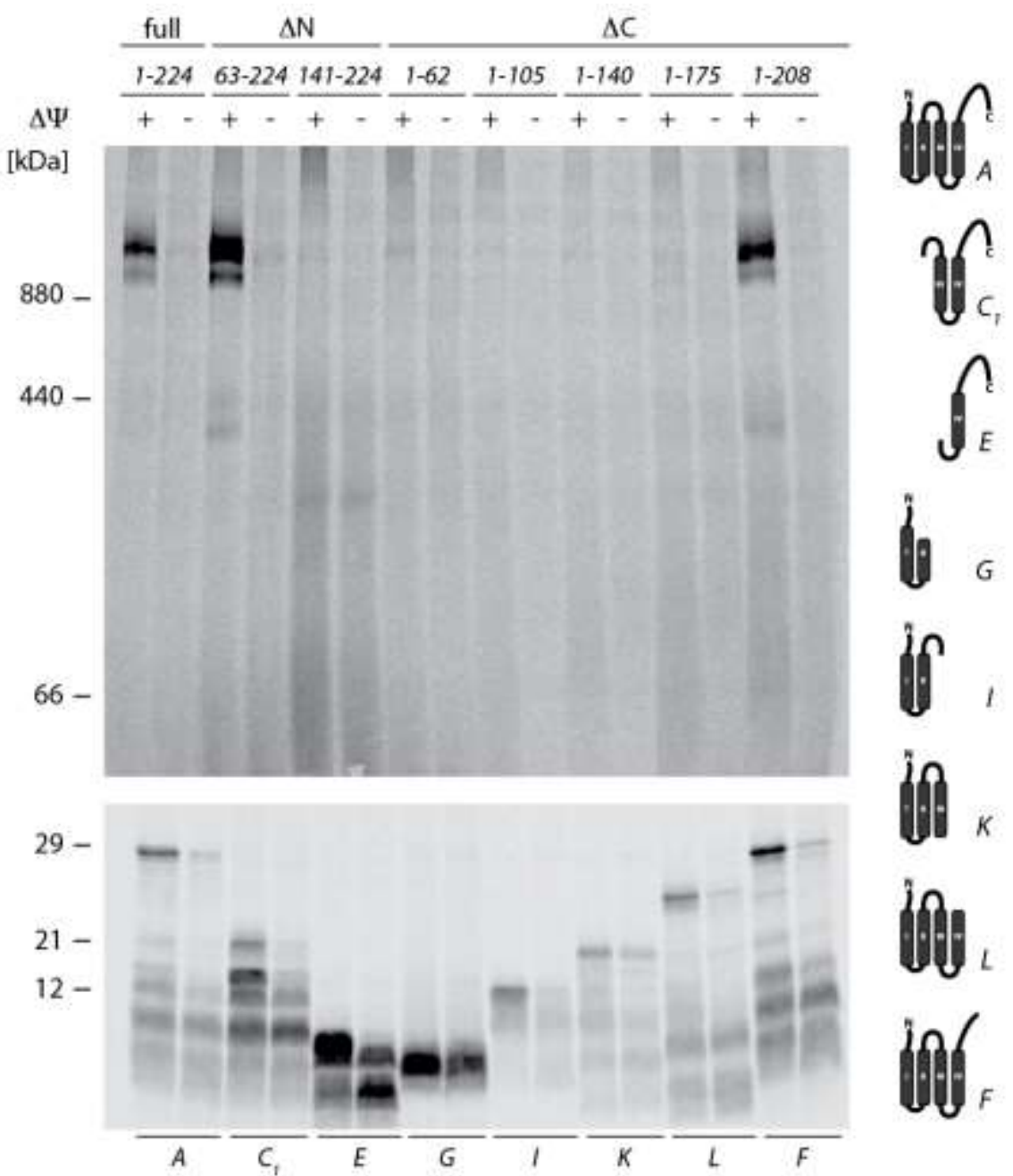

Figure 2-9: The C-terminal half of Rcf2 present in $\operatorname{Rcf}^{C}$ is essential for $\operatorname{Rcf} 2$ assembly into supercomplexes. Radiolabeled $\mathrm{N}$ - and C-terminally truncated Rcf2 constructs $(\Delta \mathrm{N}$ and $\Delta \mathrm{C})$ were imported into isolated mitochondria for $45 \mathrm{~min}$ in the presence or absence of membrane potential $(\Delta \Psi)$. Proteinase $\mathrm{K}$ treated samples were split for solubilization in $1 \%$ digitonin buffer and SDS sample buffer. Samples were analyzed by BN-PAGE or SDS-PAGE and digital autoradiography. Constructs $A, C_{1}$ and $G$ represent full length Rcf2, approximated $\operatorname{Rcf} 2^{\mathrm{C}}$ and approximated $\operatorname{Rcf} 2^{\mathrm{N}}$, respectively.

\subsubsection{Rcf2 follows an unusual import pathway into the inner membrane}

It was puzzling that $\mathrm{N}-$, as well as $\mathrm{C}-$, terminal truncations of different sizes were imported into isolated mitochondria, often even more efficiently than full length Rcf2 (Figure 2-9 lower panel). This raised the question as to how Rcf2 is imported and integrated into the membrane. The most common import pathway for the inner mitochondrial membrane is through the TIM23 machinery and usually requires an N-terminal presequence (Chacinska, Koehler, Milenkovic, Lithgow, \& Pfanner, 2009; Schatz \& Dobberstein, 1996). Such a sequence has not been predicted for Rcf2. However, an alterative pathway, via the TIM22 machinery, exists for multi-spanning 
carrier proteins of the inner membrane. Substrates of the carrier pathway usually contain four or six TMDs (Brix et al., 1999; Dudek et al., 2013; Moualij, Duyckaerts, Lamotte-Brasseur, \& Sluse, 1997). Like the known substrates of this pathway, Rcf2 contains positive charges in the matrix-exposed loops (Moualij et al., 1997; Nelson, Felix, \& Swanson, 1998). Therefore, import and assembly of Rcf2 were monitored in a temperature sensitive mutant of the small Tim protein, Tim10, which is engaged in the transfer of carrier precursors from TOM to TIM22. Upon a shift to restrictive temperature, loss of Tim10 function in tim10-2 (Truscott et al., 2002) leads to less efficient assembly of the known substrate AAC (ADP / ATP carrier). In contrast, assembly of Rcf2 into complex IV and supercomplexes remained unaffected (Figure 2-10). Hence, Rcf2 is not a substrate of the TIM22 import pathway.

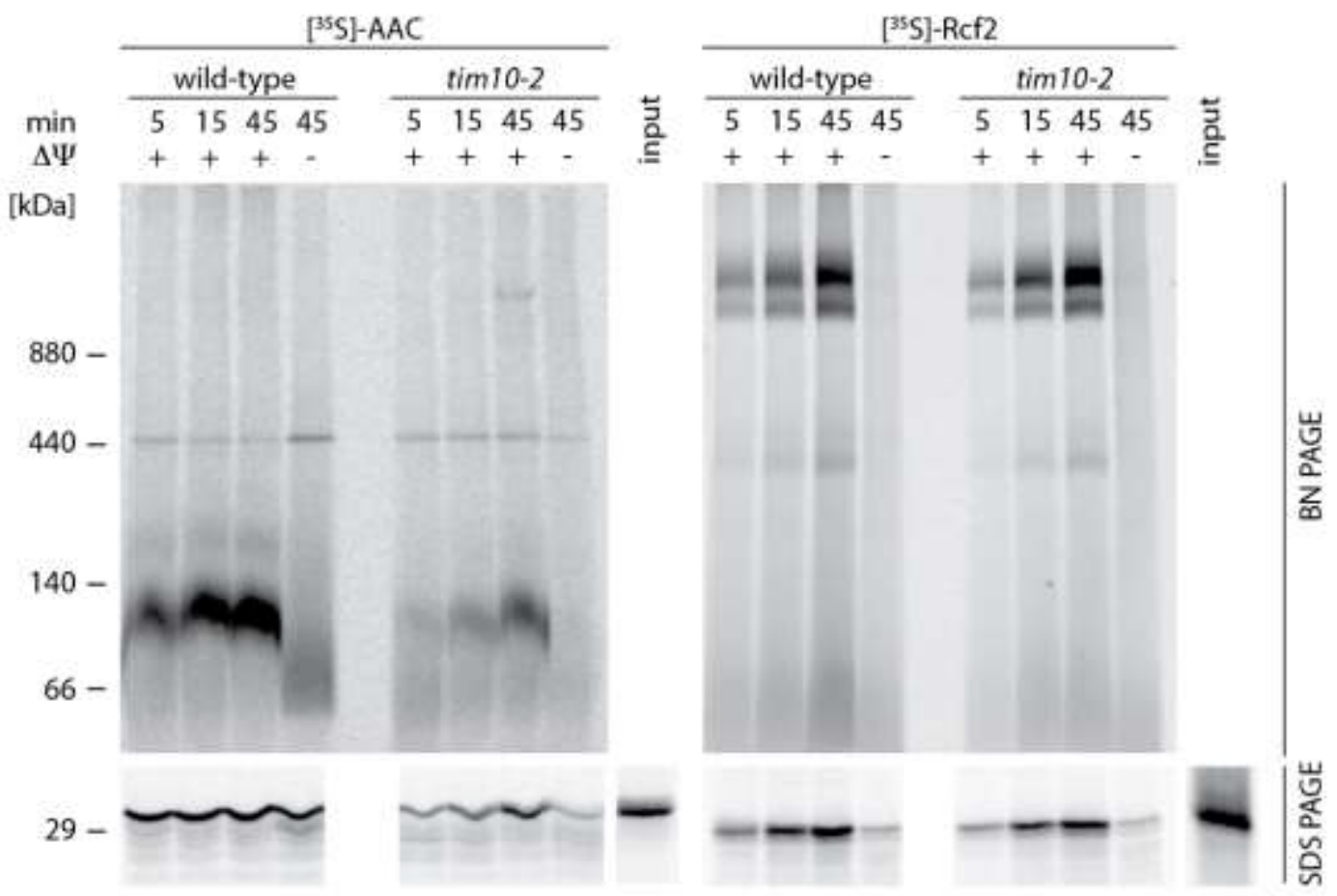

Figure 2-10: Import of Rcf2 does not rely on a functional carrier pathway. Radiolabeled AAC or Rcf2 were imported into heat-shocked isolated wild-type or tim10-2 mitochondria for the indicated times in the presence or absence of membrane potential $(\Delta \Psi)$. Proteinase $\mathrm{K}$ treated samples were split for solubilization in $1 \%$ digitonin buffer and SDS sample buffer. Samples were analyzed by BN-PAGE or SDS-PAGE and digital autoradiography.

Despite the absence of a presequence, a potential involvement of TIM23 was also tested. To this end, a genomic tim23A, ectopically expressing TIM23 under the control of a galactose-inducible promotor was used. By elimination of galactose paralleled by addition of glucose to yeast media, the levels of Tim23 can be reduced to an amount that still allow for survival of the cell but will give a strong import 


\section{RESULTS}

defect for presequence-containing proteins (Schulz et al., 2011). The import of classical substrates of the TIM23 machinery such as the inner membrane proteins Cox13 and Oxa1, and the matrix-targeted artificial construct Su9-DHFR, was severely reduced in this mutant (Figure 2-11 A). AAC does not rely on Tim23 and thus remained unaffected. For Rcf2, an initial drop in import efficiency to approximately $80 \%$ of wild-type was observed (Figure 2-11 A and B). Strikingly, after eight minutes, this reduction was completely abolished. These results do not argue for a clear-cut dependency on Tim23, however a general involvement of the presequence pathway cannot be excluded either.

A

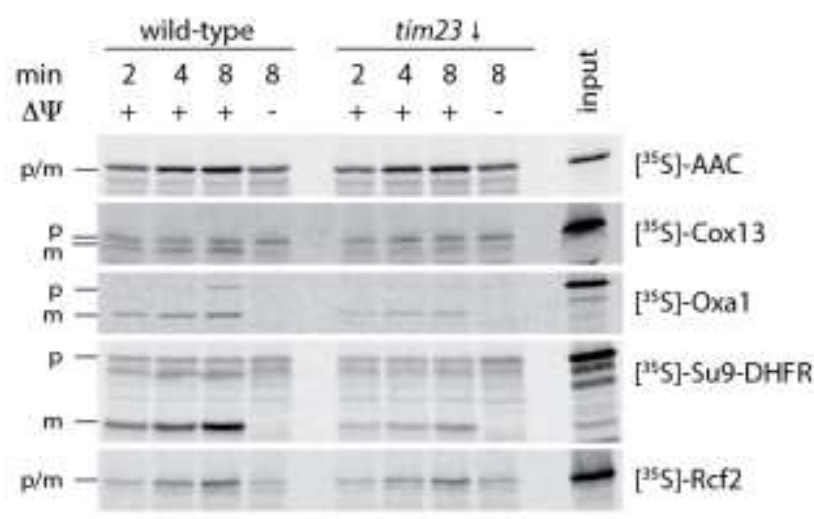

B

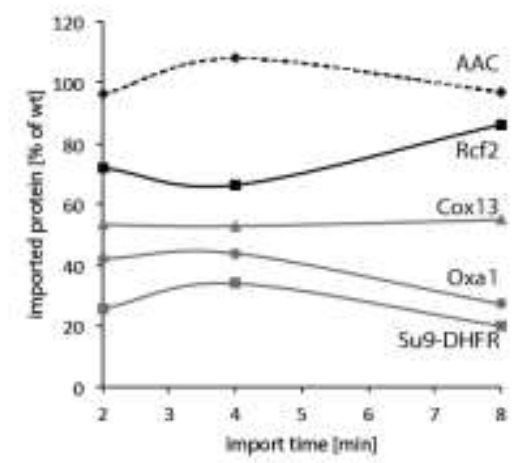

Figure 2-11: Import of Rcf2 does not strictly depend on the presence of Tim23. A) Radiolabeled precursor proteins were imported into isolated wild-type and tim23 $\downarrow$ mitochondria for the indicated times in the presence or absence of membrane potential $(\Delta \Psi)$. Proteinase $\mathrm{K}$ treated samples were lysed in SDS sample buffer and analyzed by SDS-PAGE. For better separation of pre and mature Cox13, the gel was supplemented with urea. $p=$ precursor; $m=$ mature protein $\mathbf{B}$ ) The results shown in A were quantified using ImageQuant TL software. 


\subsection{YBR255C-A / Rcf3 is a novel interaction partner of complex IV}

A valuable hint towards the potential role of the Rcf2 processing event, came from an in silico analysis in cooperation with Kay Hofmann (Institute for Genetics, University of Cologne). These alignments revealed a weak, but statistically significant, similarity of Rcf2 to Rcf1, as well as to the putative protein YBR255C-A (Figure 2-12).

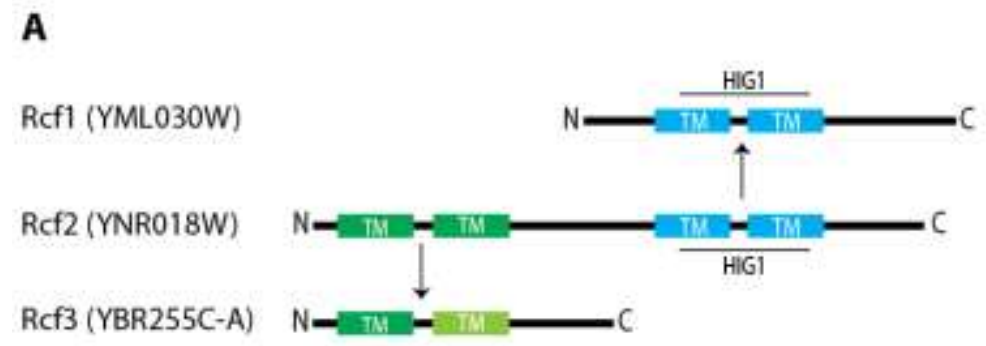

B
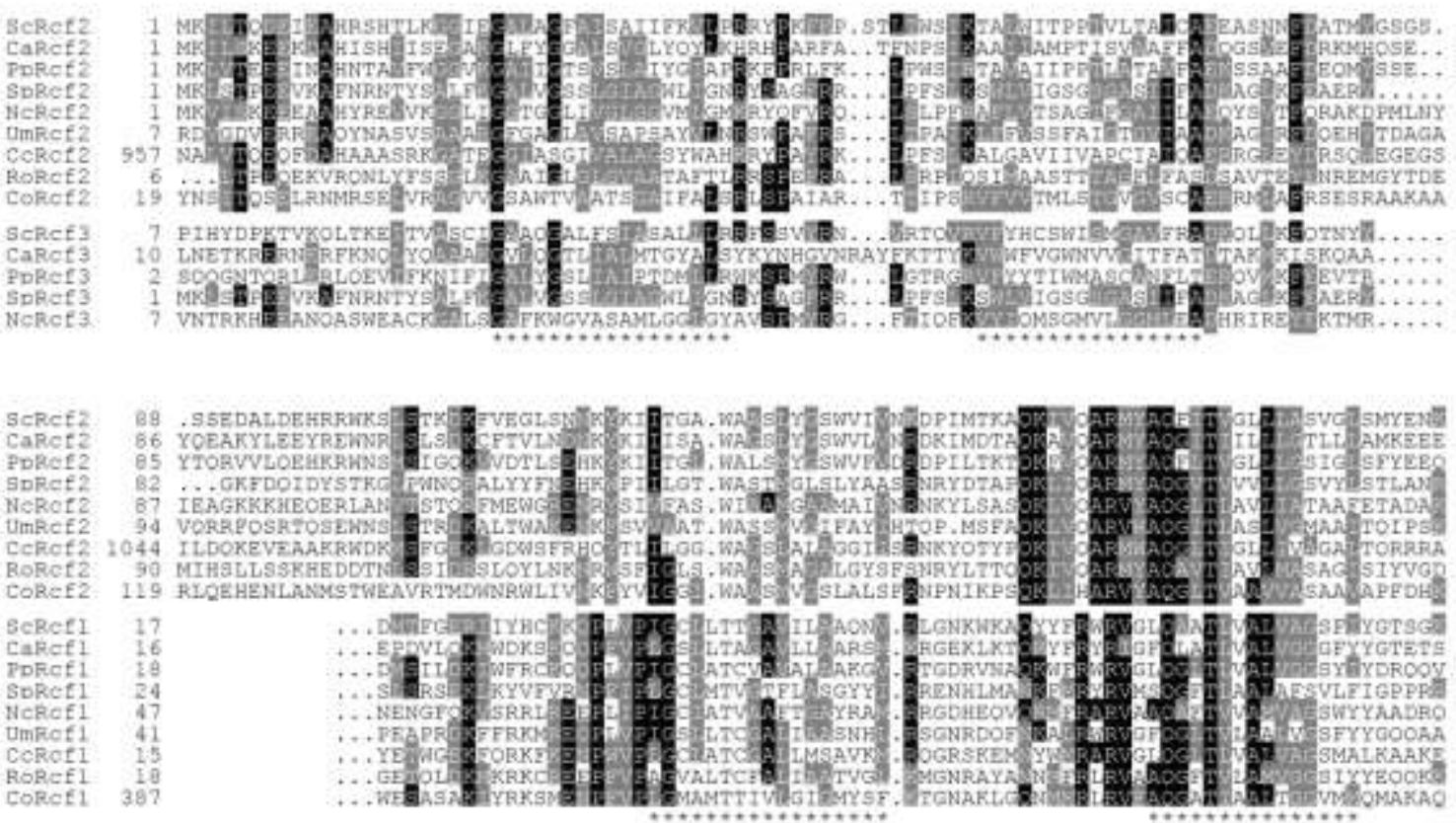

Figure 2-12: Alignment visualizing sequence similarities among Rcf1, Rcf2 and YBR255C-A (Rcf3). The model (A) was based on alignments (B) provided by Kay Hofmann (Institute for Genetics, University of Cologne). Dark green and cyan indicate transmembrane spans (TM); light green indicates a possible transmembrane span or hydrophobic patch. HIG1 marks the homology region for Hif1 $\alpha$ induced genes. Black and grey boxes indicate identical and similar residues, respectively. Asterisks mark putative transmembrane spans. Sc=Saccharomyces pombe, Ca=Candida albicans, $\mathrm{Pp}=$ Pichia pastoris, $\mathrm{Sp}=$ Schizosaccharomyces pombe, Nc=Neurospora crassa, Um=Ustilago maydis, $\mathrm{Cc}=$ Coprinopsis cinerea, $\mathrm{Ro}=$ Rhizpus oryzae, $\mathrm{Co}=$ Capsaspora owczarzaki 
It had previously been suggested that Rcf1 and Rcf2 might be related to each other (Strogolova et al., 2012) and this data now clearly assigns the similarity region to the C-terminal half of Rcf2. In contrast, the similarity region for YBR255C-A falls within the N-terminal half of Rcf2. Since there is almost no overlap, Rcf1 and YBR255C-A could be envisioned as a split paralogue of Rcf2.

Due to these observations, the so far uncharacterized protein product of YBR225C-A was named Rcf3. A mitochondrial targeting signal could not be predicted. Nevertheless, the protein had been suggested to interact with complex III / IV in a proteomics-based study by Helbig and coworkers (Helbig et al., 2009). Most interestingly, in the same study, a complex III / IV association of Rcf1 and Rcf2 was predicted, which was later on, confirmed biochemically (Chen et al., 2012; Strogolova et al., 2012; Vukotic et al., 2012).

An in depth analysis of Rcf3 therefore seemed to be of value for understanding the role of the Rcf family.

\subsubsection{Rcf3 is a protein of the inner mitochondrial membrane}

Mitochondrial localization of Rcf3 was confirmed in a strain expressing a C-terminal GFP-tagged Rcf3. Rcf3GFP colocalized with the mitochondrial network, visualized using MitoTracker in living yeast cells (Figure 2-13 A). Microscopic analysis was kindly provided by Markus Deckers (Department of Cellular Biochemistry, University Medical Center Göttingen). The open reading frame furthermore contains an intron ranging from G64 to G157 that is removed from the mRNA prior to translation, leading to a protein of approximately $14 \mathrm{kDa}$.

Based on the predicted model (Figure 2-12), Rcf3 should contain one to two TMDs. Consistently, it behaved like the integral inner membrane proteins Tim21 in a carbonate extraction analysis (Figure 2-13 B). Even in a carbonate solution of pH 11.5, Rcf3 exclusively remained in the membrane fraction.

To determine in which of the mitochondrial membranes Rcf3 is located, wild-type mitochondria were tested for the accessibility of protease to Rcf3 in mitoplasts and in intact mitochondria (Figure 2-13 C). Rcf3 was stable in proteinase K-treated mitochondria. Only upon removal of the outer membrane the Rcf3 signal decreased in a similar manner to Tim23 (inner membrane) but not to Tom70 (outer membrane), or Tim44 (matrix). The decreasing signal indicates a protease 
accessibility of the C-terminal antibody epitope. Hence, the protein should localize to the inner membrane, with its C-terminus facing the IMS.
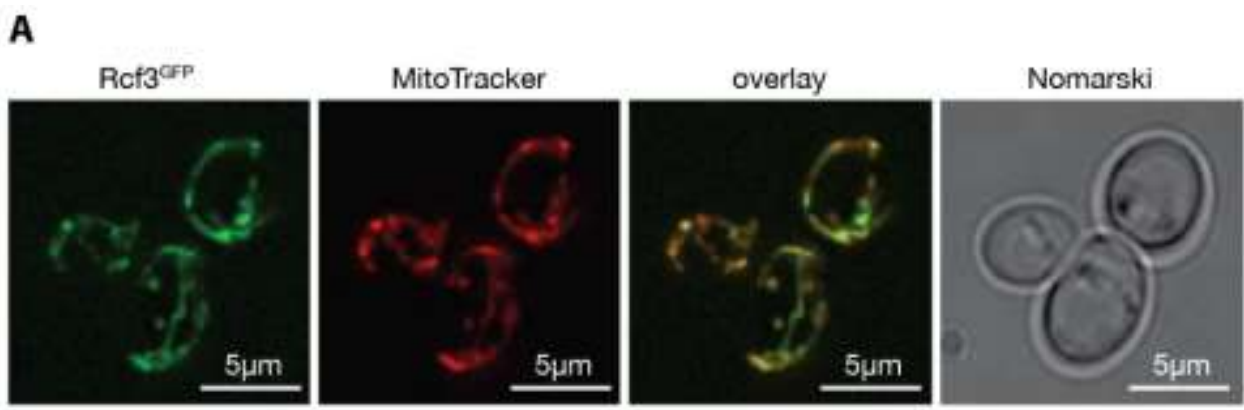

B

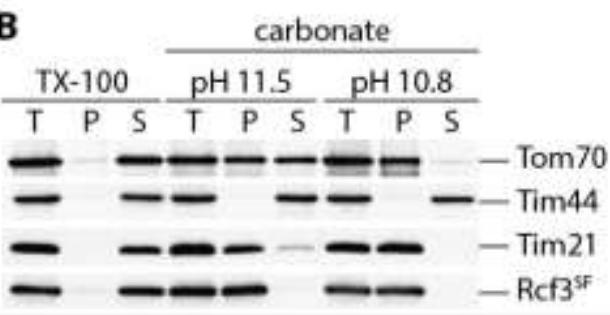

C

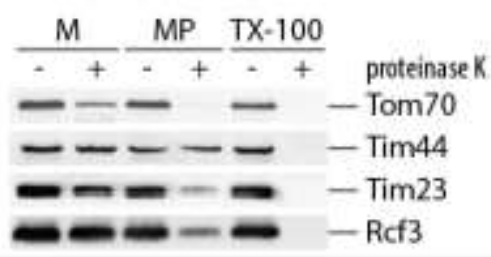

Figure 2-13: Rcf3 localizes to the inner mitochondrial membrane and exposes its C-terminus to the IMS. A) Distribution of Rcf3 was analyzed by fluorescence microscopy in cells expressing Rcf3GFP. To test for colocalization, mitochondria were visualized using MitoTracker. Scale bar, $5 \mu \mathrm{m}$. B) Mitochondria of Rcf3 ${ }^{\mathrm{SF}}$ expressing cells were subjected to carbonate extraction or lysed with $1 \%$ Triton X-100 (TX-100). Using ultra centrifugation, samples were separated into pellet (P) and supernatant $(\mathrm{S})$ and compared to total $(\mathrm{T})$. Samples were subjected to western blot analysis with Tom70 and Tim21 as membrane bound controls and Tim44 as a soluble control. C) Wild-type mitochondria were left untreated (M), swollen (MP) or lysed with $1 \%$ Triton X-100, treated with proteinase K where indicated and subjected to SDS-PAGE and western blotting. Tom70, Tim23 and Tim44 served as controls for protease-accessible, OM-protected and IM-protected proteins, respectively.

At this point, it remains elusive as to whether Rcf3 contains only one TMD, or two. Based on the low hydrophobic profile of TMD2 in the hydrophobicity plot, it might well be that this region represents a hydrophobic patch rather than a true transmembrane span.

\subsubsection{Rcf3 is not essential for respiration but interacts with respiratory supercomplexes}

The complex IV association predicted by Helbig and coworkers (Helbig et al., 2009) raised the question as to whether Rcf3 might be important for the cells ability to respire. Therefore, RCF3 was genomically deleted. The strain was tested for growth on non-fermentable media in comparison to a respiration-deficient cox5a $\Delta$ strain. Unlike $r c f 1 \Delta, r c f 3 \Delta$ showed no respiratory defect on lactate medium (Figure 2-14 A), thereby resembling $r c f 2 \Delta$. In line with this, a BN-PAGE analysis of mitochondria 
isolated from $r c f 3 \Delta$ did not reveal a reorganization of respiratory supercomplexes, especially of complex IV (Figure 2-14 B). Neither in vitro activity assays for complex III / IV (Figure 2-14 C), nor oxgygen consumption measurements with intact mitochondria (Figure 2-14 D), revealed a defect for $r c f 3 \Delta$.

Nevertheless, the deletion strain provided a useful tool for the import and assembly of radiolabeled Rcf3 precursor. When energized $r c f 3 \Delta$ mitochondria were incubated with [ $\left.{ }^{35} \mathrm{~S}\right]$-Rcf3 or [ $\left.{ }^{35} \mathrm{~S}\right]$-Rcf1 and subsequently solubilized in digitonin, both proteins were strongly detectable in respiratory supercomplexes (Figure 2-15 A). Additionally, complexes in the range of monomeric complex IV (IV Dig) were detected, as well as an Rcf3-specific complex of approximately $100 \mathrm{kDa}$. In contrast, in wild-type mitochondria analyzed in the same way, Rcf3 was barely detectable (data not shown). While supercomplexes are generally preserved in digitonin, the harsher detergent DDM disintegrates them into their individual counterparts. Upon solubilization in DDM, only residual amounts of both proteins remained in complex. In the case of Rcf1, this complex is known to correspond to complex IV, which migrates slightly faster in DDM (IV $V_{\text {DDM }}$ ) than in digitonin (IV $V_{\text {Dig }}$. The barely visible Rcf3 complex in DDM however seemed to run slightly slower than IV $V_{\text {DDM. }}$ 


\section{RESULTS}

A

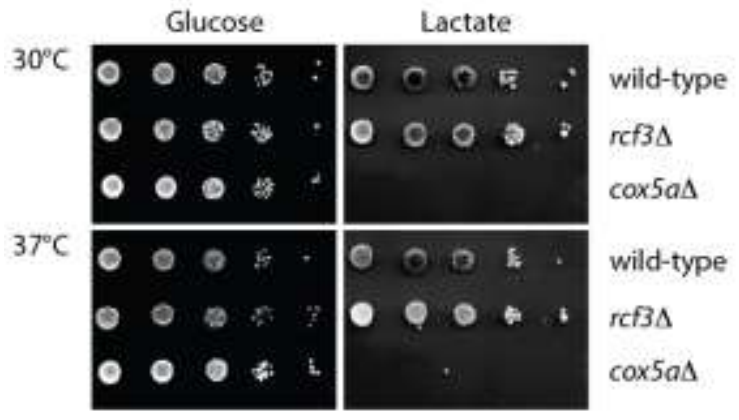

B

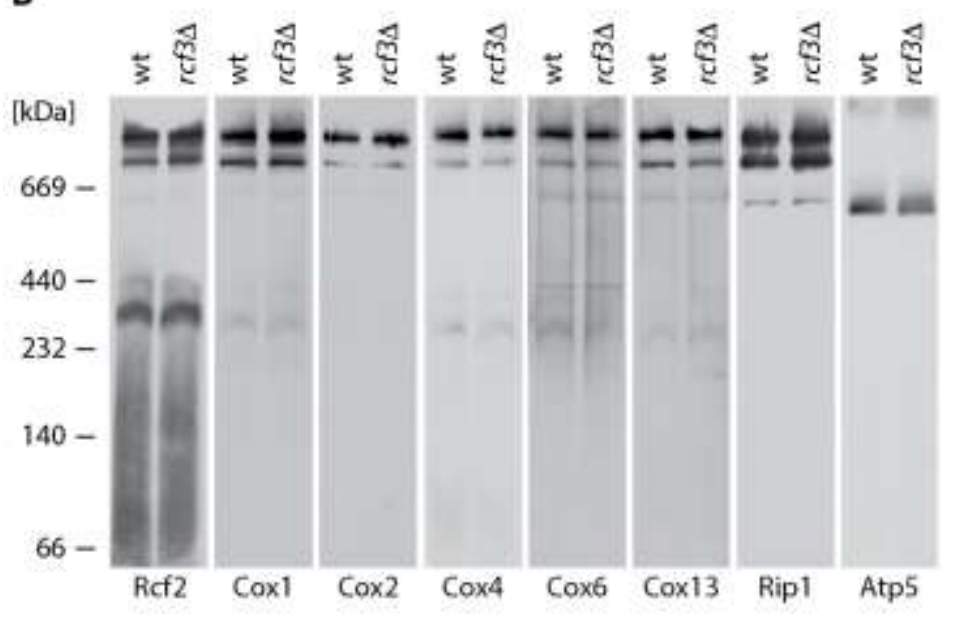

C
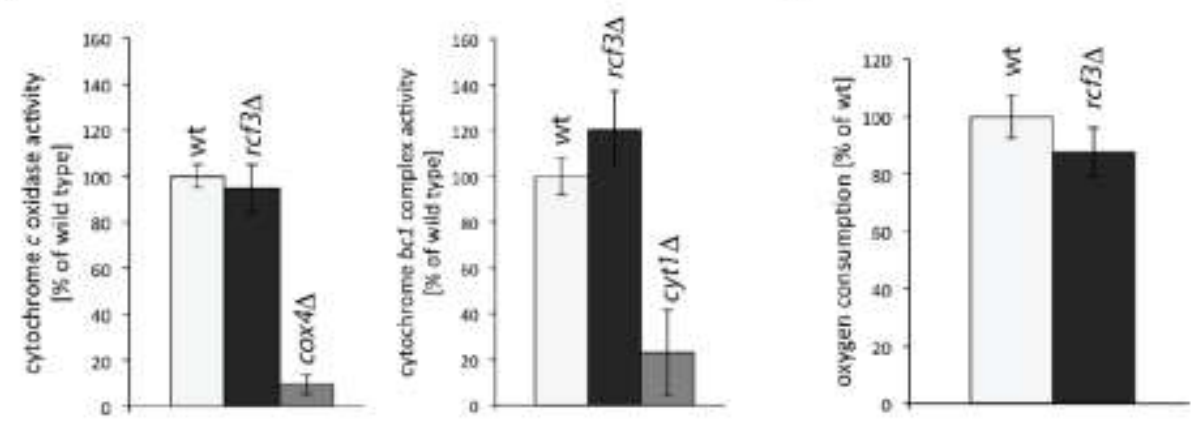

Figure 2-14: Deletion of Rcf3 does not affect respiration. A) $r c f 3 \Delta$ cells were tested for growth on minimal media supplemented with glucose or lactate at the indicated temperatures in comparison to wild-type and respiratory deficient $\operatorname{cox} 5 a \Delta$. B) Digitonin-solubilized mitochondria of wild-type and rcf3 $\Delta$ were analyzed by BN-PAGE and western blotting. Antibodies against Rcf2, Cox1, Cox2, Cox4, Cox6 and Cox13 were used to detect complex IV. Complex III was detected via Rip1, and ATPase via Atp5. C) Cytochrome $b c_{1}$ complex and cytochrome $c$ oxidase activity were measured in isolated mitochondria of wild-type, cox $4 \Delta$, cyt1 $\Delta$ and $\operatorname{rcf} 3 \Delta$ (mean of $n=6 / n=4 \pm$ STDEV). D) Oxygen consumption rates were measured in isolated wild-type and $r c f 3 \Delta$ mitochondria using an Oxygraph $2 \mathrm{k}$ (Oroboros) at $30^{\circ} \mathrm{C}$ (mean of $\left.\mathrm{n}=4 \pm \mathrm{STDEV}\right)$.

For a direct comparison of Rcf3- and Rcf1-containing complexes in both detergents, samples were loaded in adjacent lanes (Figure 2-15 B - autoradiogram). This clearly proved that, apart from supercomplex structures, all Rcf3 complexes were slightly bigger than the corresponding Rcf1-containing complexes. To ensure that the size of 


\section{RESULTS}

the latter was identical with complex IV, mitochondrial lysates were probed for Cox1 and Rip1 (Figure 2-15 B - western blot). The results thus indicate a supercomplex association of Rcf3. However, whether this association is mediated through complex IV, could not be answered using this experimental setup.
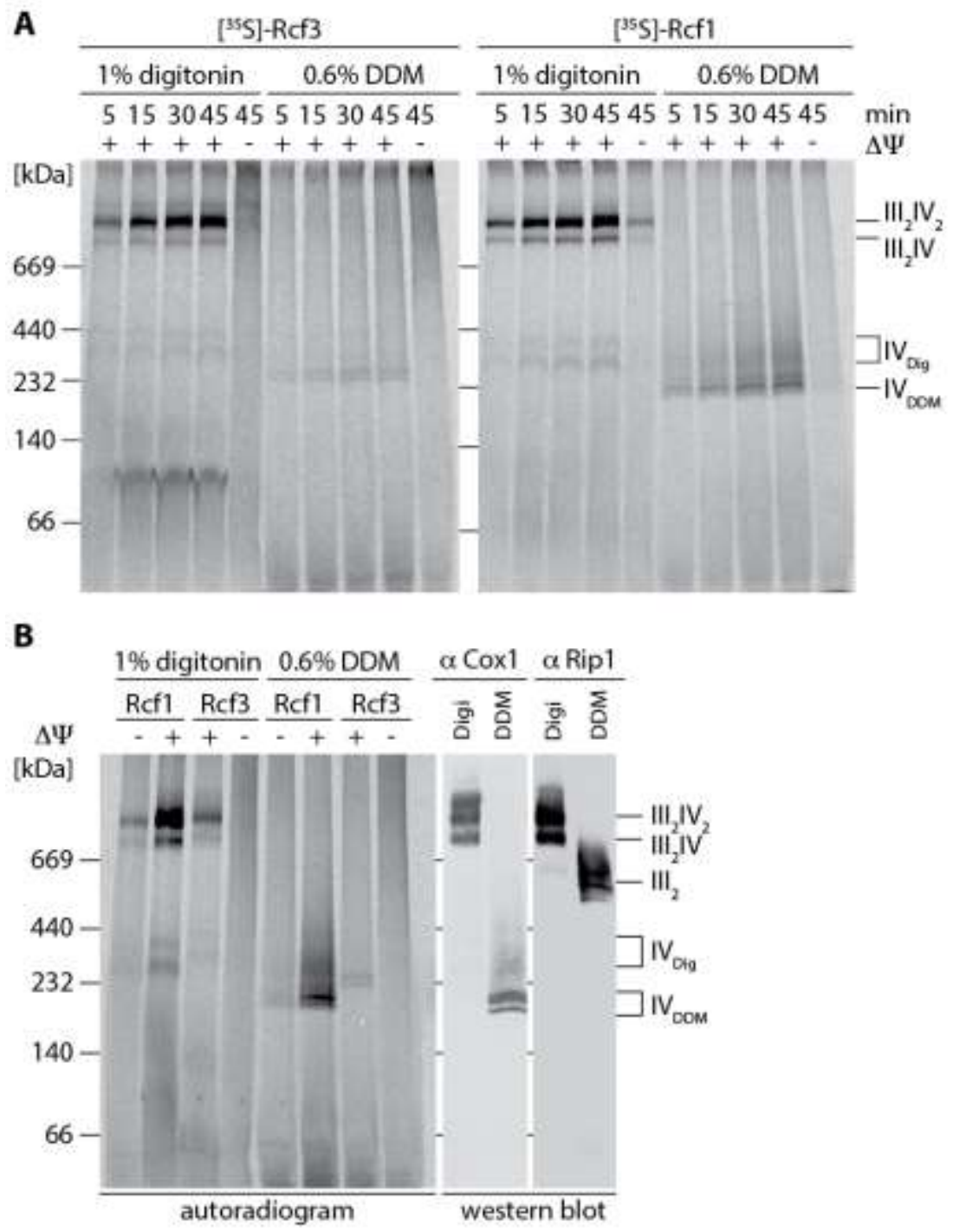

Figure 2-15: Rcf3 is assembled into respiratory supercomplexes in isolated $r c f 3 \Delta$ mitochondria. A) Radiolabeled Rcf3 or Rcf1 were imported into isolated $r c f 3 \Delta$ mitochondria for the indicated times in the presence or absence of membrane potential $(\Delta \Psi)$ ). Proteinase $K$ treated samples were then solubilized in $1 \%$ digitonin or $0.6 \%$ DDM and analyzed by BN-PAGE and digital autoradiography. $I V_{\text {Dig }}$ and $I_{\mathrm{DDM}}$ indicate the size of the Rcf1-containing pool of monomeric complex IV when solubilized in the respective detergent. B) Radiolabeled Rcf3 or Rcf1 were imported into isolated $r c f 3 \Delta$ mitochondria for $45 \mathrm{~min}$ and analyzed as specified in A. For comparison, wild-type mitochondria were solubilized in 1\% digitonin or $0.6 \%$ DDM and analyzed by BN-PAGE and western blotting. Complex III and IV containing assemblies were visualized by immunodetection of Rip1 and Cox1, respectively. 


\subsubsection{The supercomplex association of Rcf3 is mediated through complex IV and complex III}

A possibility to distinguish between complex IV and complex III-mediated supercomplex association is provided by the isolation of a ZZ-tagged subunit of either complex (Cox4ZZ or Cor $1^{\mathrm{ZZ}}$ ). In digitonin-solubilized mitochondria, both proteins reside within and hence interact with supercomplexes. In DDM however, Cox 4 and Cor1 exclusively coisolate subunits of individual complex IV and III respectively. Small amounts of Rcf3 were isolated with both complexes in digitonin, confirming a supercomplex association of the protein that was observed in the previous experiment (Figure 2-16 A). Again, DDM treatment caused almost complete dissociation of Rcf3. Consistently only digitonin-solubilized Rcf3ZZ was able to copurify small amounts of both complexes (Cox1, Cox2, Rcf1, Rcf2 as well as Rip1) in the inverse experiment (Figure 2-16 B). Interestingly, DDM abolished all interactions except the one between Rcf3 ${ }^{Z Z}$ and Rcf1, which was weakened but still detectable.

A

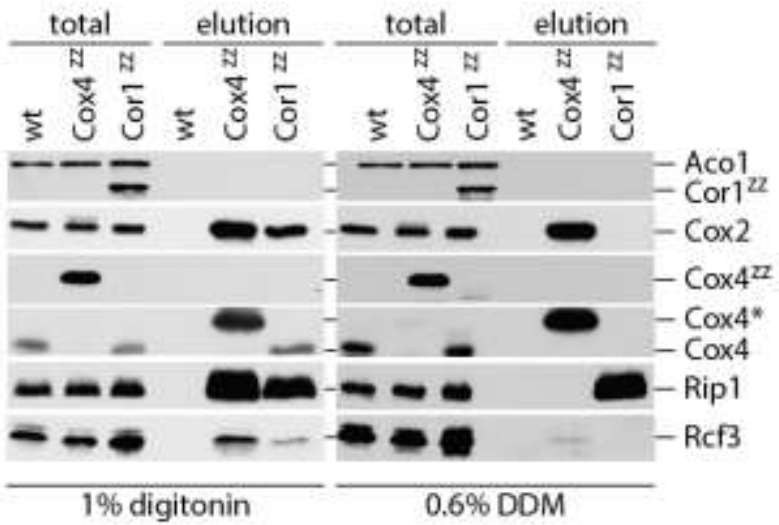

B

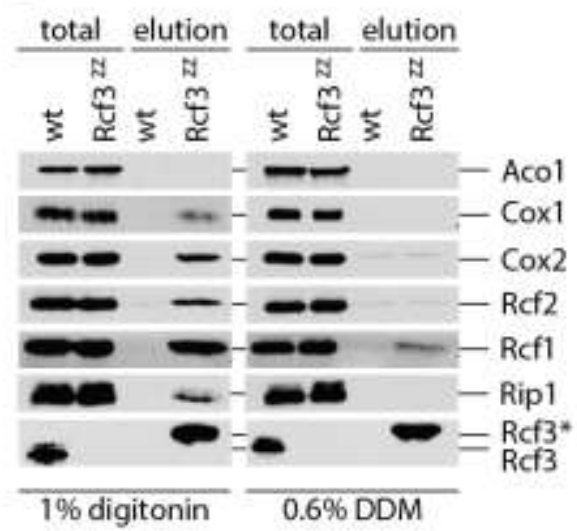

Figure 2-16: Endogenous Rcf3 interacts with supercomplexes but dissociates in DDM. A) Mitochondria isolated from wild-type (wt), Cox $4^{\mathrm{ZZ}}$ and Cor1 ${ }^{\mathrm{ZZ}}$ strains were solubilized in $1 \%$ digitonin or $0.6 \%$ DDM and subjected to IgG chromatography. Upon TEV protease cleavage, elutions were analyzed by SDS-PAGE and western blotting. Cox $4^{*}$ marks Cox 4 after removal of ZZ by TEV cleavage (total: 5\%; elution: 100\%). B) Mitochondria isolated from wild-type (wt) and Rcf3zz were treated as in A. Cox4*/ Rcf3* mark Cox4/Rcf3 after removal of ZZ by TEV cleavage (total: 5\%; elution: $100 \%)$.

Due to the labile nature of Rcf3 in DDM, discrimination between complex III and IV association was still not possible although Rcf3 seemed to be slightly more abundant in Cox4zZ elutions. In summary, Figures 2-15 and 2-16 therefore prompted the speculation that Rcf3 is loosely associated with the oxidase. It has to be questioned whether such a labile interaction partner would be stably associated during other 
analyses such as BN-PAGE. The cooperation of the neutral detergent digitonin and the anionic Coomassie dye had already been reported to create harsher conditions than originally anticipated (Schägger, 2001). To circumvent a potential dissociation of Rcf3-containing assemblies during BN-PAGE, a gel filtration analysis was performed (Figure 2-17). In digitonin-solubilized wild-type mitochondria only a fraction of Rcf3 comigrated with respiratory supercomplexes (indicated by Cox1 and Rip1), while the majority accumulated in fractions with lower molecular weight (Rcf3small). This result fits well into the observation that only small amounts of Rcf3 were coisolated with Cox4ZZ and Cor1ZZ (Figure 2-16 A).
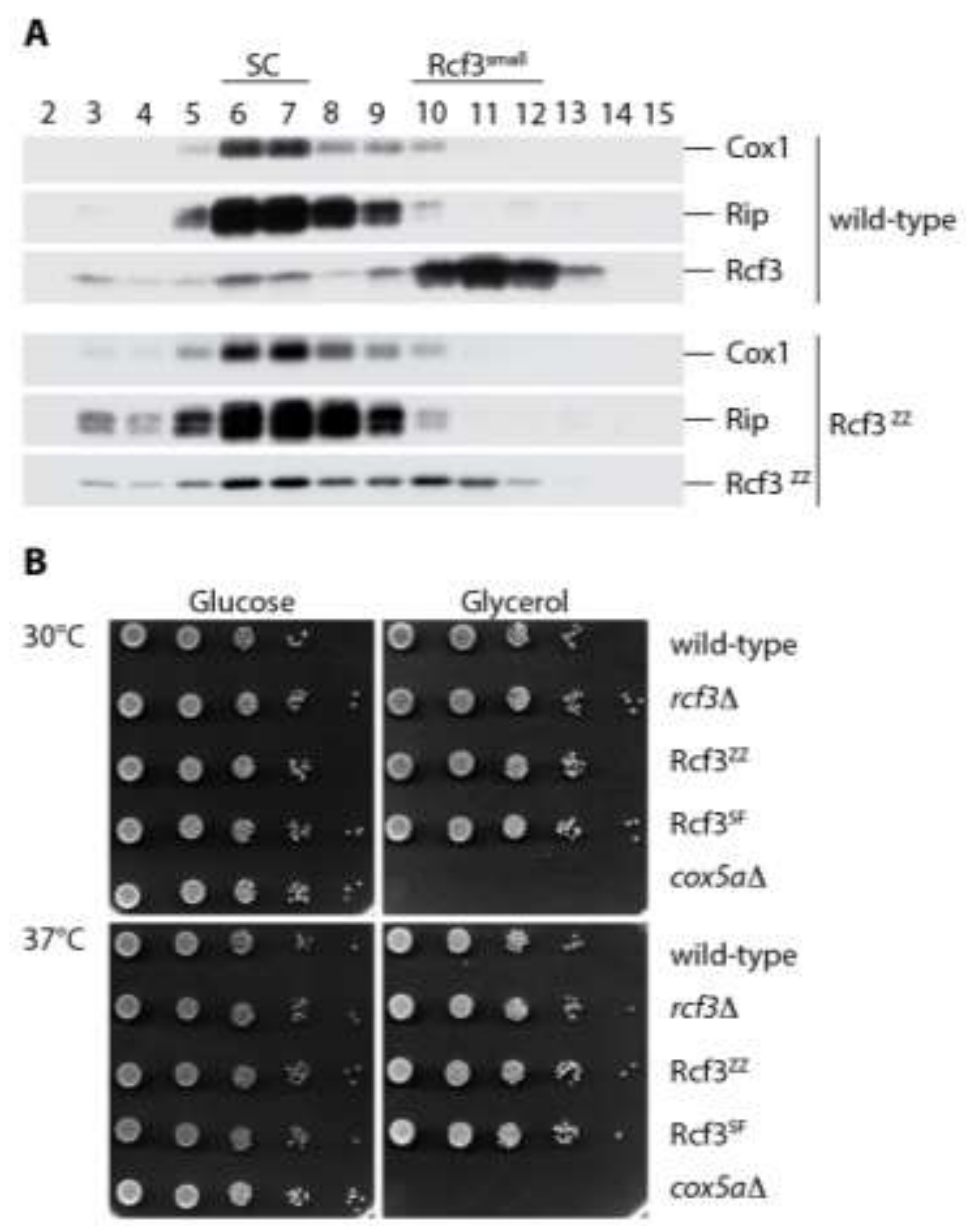

Figure 2-17: C-terminal ZZ tagging alters supercomplex association of Rcf3 even though respiration remains unaffected. A) Digitonin-solubilized mitochondria from wild-type and Rcf3ZZ were subjected to gel filtration analysis. Fractions 2-15 were precipitated with the help of TCA and subsequently analyzed by SDS-PAGE and western blotting. Cox1 and Rip1 indicate fractions containing supercomplexes (SC). B) $r c f 3 \Delta$, Rcf3 ${ }^{Z Z}$ and Rcf3 ${ }^{S F}$ cells were tested for growth on minimal media supplemented with glucose or glycerol at indicated temperatures in comparison to wild-type and respiratory deficient cox $5 a \Delta$.

In contrast, the addition of a C-terminal ZZ tag led to increased supercomplex association, with almost equal amounts of Rcf3ZZ in the supercomplex and the 
Rcf3small pool (Figure 2-17 A). This effect was surprising, especially since the addition of tags did not affect respiration in a growth test (Figure 2-17 B). In light of the tag-related reorganization of supercomplexes, the Rcf3ZZ strain is not an appropriate tool. Hence the antibody against the endogenous protein was tested for immunoprecipitation and found to be suitable for the enrichment of Rcf3 from digitonin-solubilized mitochondria. To distinguish between complex III and complex IV association in an unambiguous way, Rcf3 was precipitated from $\operatorname{cox} 4 \Delta$ and cyt1 $1 \Delta$. Mitochondria from these strains do not contain fully assembled complex IV and III, respectively and so all observed interactions should thus be independent from respiratory supercomplexes. As expected, endogenous Rcf3 coisolated small amounts of Cox1 and Cox2 (complex IV), as well as Cyt1, Rip1 and Qcr8 (complex III) in wild-type (Figure 2-18). In the absence of complex III, Rcf3 still copurified Cox1 and Cox2 and likewise, it brought all tested complex III subunits in the absence of complex IV. This questions the hypothesized model, rather suggesting that Rcf3 localizes to an interface without clear assignment to complex III or IV.

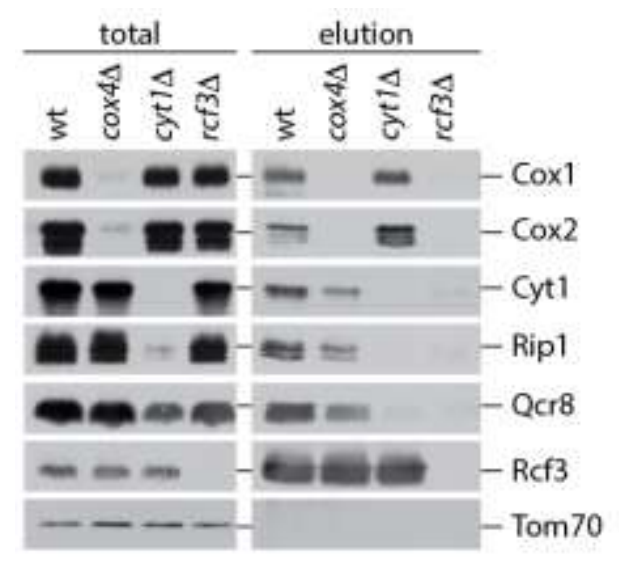

Figure 2-18: Rcf3 is able to interact with both complex III and complex IV. Digitonin-solubilized mitochondria from wild-type (wt), cox $4 \Delta$, cyt1 $\Delta$ and rcf3 $\Delta$ were used for immunoprecipitation of Rcf3. Totals (3\%) and elutions (100\%) were analyzed by SDS-PAGE and western blotting.

\subsubsection{Rcf proteins accumulate in small complexes}

Both gel filtration (Figure 2-17) and coimmunoprecipitation experiments (Figure 2-18) concordantly showed that only a minor fraction of Rcf3 associates with respiratory supercomplexes. Even though originally described as complex IV subunits, closer analysis of Rcf1 and Rcf2 revealed these two proteins have very similar characteristics. At least Rcf1 coisolates only small amounts of complex III and IV, as judged by detection of Cox2 and Qcr8 (Figure 2-19 A). This seems to be 


\section{RESULTS}

the case for Rcf2 as well, although an estimation of the levels of coisolated proteins is aggravated by unequal isolation efficiency.
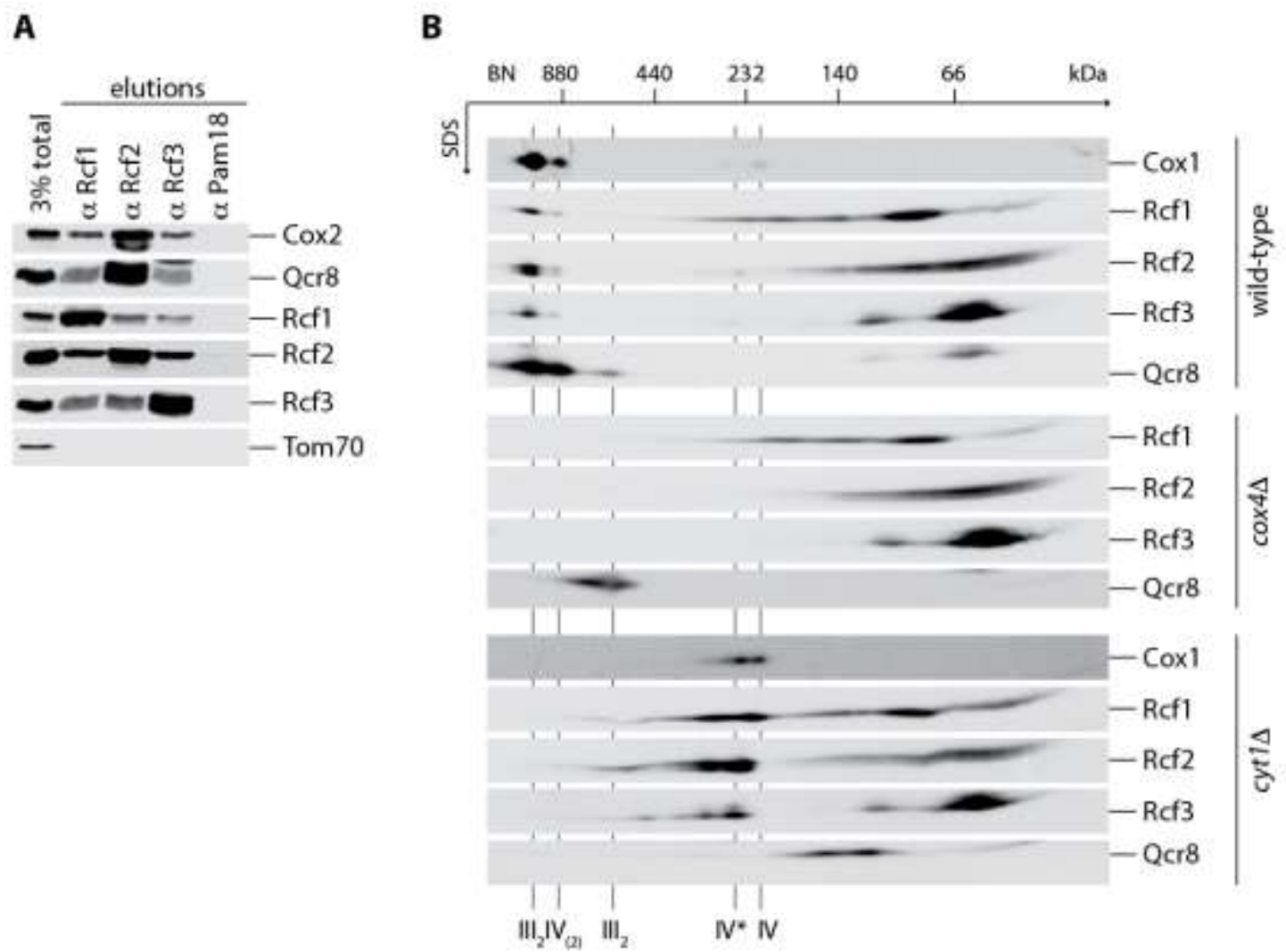

Figure 2-19: All Rcf proteins accumulate in small assemblies independent of complex III / IV. A) Digitonin-solubilized wild-type mitochondria were used for immunoprecipitation of Rcf1, Rcf2, and Rcf3. Pam18 was used as a negative control. Totals and eluates were analyzed by SDS-PAGE and western blotting. B) Digitonin-solubilized mitochondria of wild-type, cox $4 \Delta$ and $c y t 1 \Delta$ were analyzed by BN-PAGE, followed by a second dimension SDS-PAGE and western blotting. Cox1 and Qcr8 indicate the positions of respiratory supercomplexes $\left(\mathrm{III}_{2} \mathrm{IV}_{2}\right.$ and $\left.\mathrm{III}_{2} \mathrm{IV}\right)$, complex $\mathrm{III}_{2}$ and complexes IV / IV*.

To examine the presence of small assemblies, similar to those observed for Rcf3, wild-type mitochondria were subjected to second-dimension PAGE analysis and probed for all three Rcf proteins. This analysis indeed revealed distinct small Rcf complexes in addition to the known supercomplex associations (Figure 2-19 B). Moreover, these complexes were also formed to the same extent and size in the absence of fully assembled complex III (cyt1 $\Delta$ ) or complex IV (cox4A) (Figure 2-19 B). Judging from migration behavior, Rcf1 seems to form a very prominent $100 \mathrm{kDa}$ complex on its own, while Rcf2 and Rcf3 could potentially reside within a joint complex. The latter runs at approximately $70 \mathrm{kDa}$ and contains the bulk of Rcf3. In contrast to data shown in Figure 2-18, the analysis shown in Figure 2-19 B once 


\section{RESULTS}

again supports an exclusive complex IV association. In cyt1 $\Delta, \operatorname{Rcf} 3$ comigrated with Rcf1 and Rcf2 in the range of complex IV*, but not complex IV. Compared to Rcf1 and Rcf2, it seemed to be low in abundance since this interaction was barely visible in wild-type mitochondria. In cox4 $\Delta$ on the other hand, no Rcf2 and Rcf3 assemblies beyond the size of the small Rcf complexes were detected, excluding a complex III association. Only Rcf1 still appeared in a range up to $240 \mathrm{kDa}$.

\subsubsection{Attempts to define the role of the small Rcf-containing complexes}

Assuming that Rcf2 and Rcf3 reside within one small complex it should be possible to show a supercomplex-independent interaction between the two proteins. As is apparent in earlier experiments, most Rcf interactions are lost in DDM. Therefore, analysis of $\operatorname{cox} 4 \Delta$ and cyt1 $1 \Delta$ was pursued. Immunoprecipitation of Rcf1, Rcf2 and Rcf3 revealed interactions between these proteins that are indeed independent of the presence of fully assembled respiratory supercomplexes (Figure 2-20 A). Like Rcf3, Rcf1 and Rcf2 were both able to copurify stable, albeit reduced, amounts of complex III in the absence of complex IV. The absence of fully assembled complexes in these mutants is based on a stalled assembly due to the lack of a structural subunit (Cox4/ Cyt1). Remaining present, however, are assembly intermediates that might still provide a platform for a complex III / IV-specific interaction. Even though in such case an increase in Rcf-containing small complexes in Figure 2-19 B could be anticipated, this option was tested by an additional experiment. To prevent the formation of assembly intermediates, mitochondria were isolated from strains lacking mitochondrial DNA. Without the catalytic core subunits encoded in the mitochondrial genome, the assembly of respiratory complexes is entirely disrupted. Nevertheless, Rcf3 still coisolated small amounts of Rcf2 and even increased amounts of Rcf1 (Figure 2-20 B), strongly pointing towards a pre-assembly interaction. 


\section{RESULTS}

A

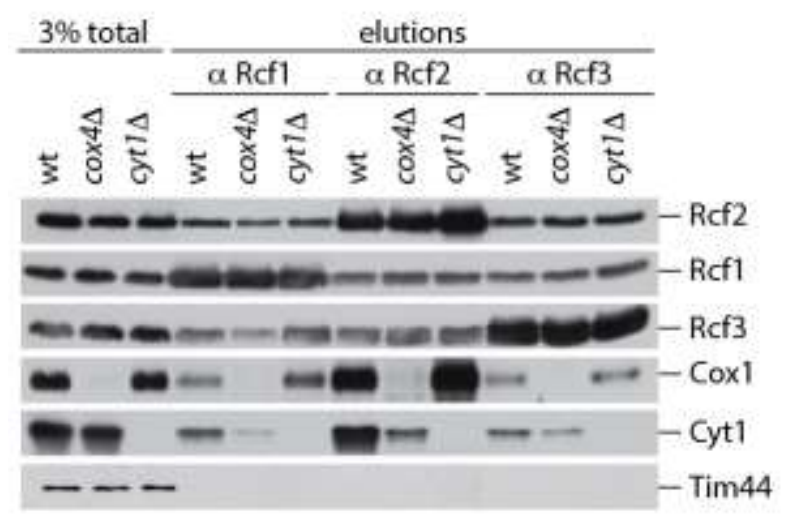

B

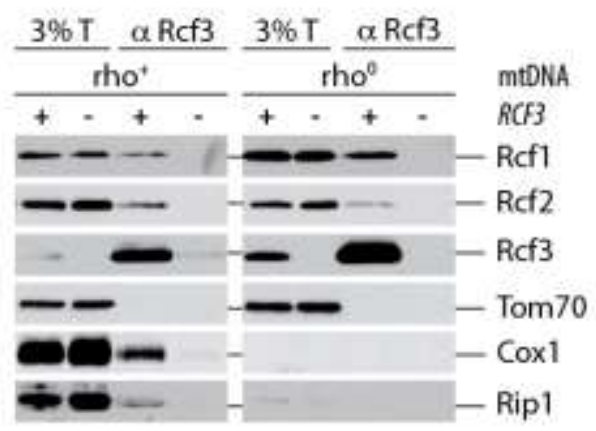

Figure 2-20: Rcf proteins interact with each other independently of complex III or IV. A) Digitonin-solubilized mitochondria from wild-type (wt), $\operatorname{cox} 4 \Delta$ and cyt1 $\Delta$ were used for immunoprecipitation of Rcf1, Rcf2, and Rcf3. Totals and eluates were analyzed by SDS-PAGE and western blotting. B) Digitonin-solubilized mitochondria from wild-type $(+)$ and $r c f 3 \Delta(-)$ either with $\left(\mathrm{rho}^{+}\right)$or without $\left(\mathrm{rho}^{0}\right)$ mitochondrial DNA were used for immunoprecipitation of Rcf3 and further processed as in $\mathrm{A}$.

Consequentially, it had to be tested whether this interaction truly takes place within the observed small complexes. Loss of an interaction partner in $r c f 1 \Delta, r c f 2 \Delta$ or $r c f 3 \Delta$ was assumed to trigger disintegration, or at least a size shift of small complexes. However, the organization of small complexes did not change in the expected way in a two dimensional analysis. Rcf1 and Rcf2 stably maintained their distribution pattern in the absence of the other respective Rcfs (Figure 2-21). Rcf3 appeared to be unchanged in the absence of Rcf2 but slightly rearranged in $r c f 1 \Delta$. It usually forms two distinct small complexes: R3a ( $\sim 140 \mathrm{kDa})$ and R3b $(\sim 70 \mathrm{kDa})$. While R3b remained unchanged, R3a seemed to be increased (Figure 2-21 - rcf1 $\Delta$ ). At the same time, the supercomplex association of Rcf3 was weakened. It has to be noted in this context that Rcf2 was strongly detected in supercomplexes in $\operatorname{rcf} 1 \Delta$ even though it had been reported to assemble less efficiently in the absence of Rcf1 (Vukotic et al., 2012). 


\section{RESULTS}

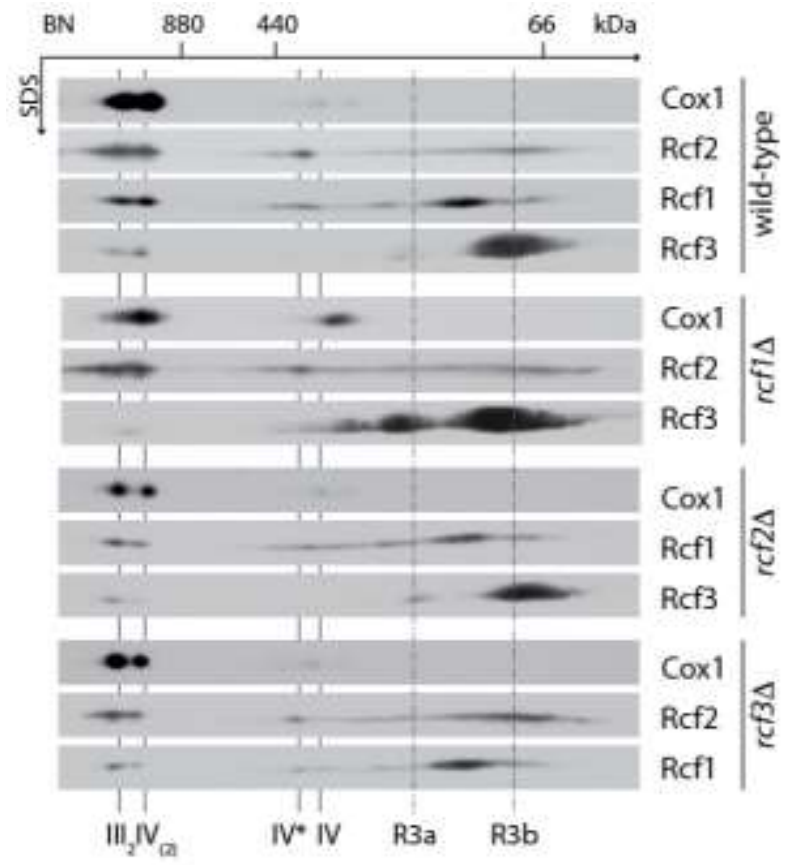

Figure 2-21: The small Rcf complexes form independently of the presence of the other Rcfs. Digitonin-solubilized mitochondria of wild-type, $r c f 1 \Delta, r c f 2 \Delta$ and $r c f 3 \Delta$ were analyzed by BN-PAGE followed by a second dimension SDS-PAGE and western blotting. Cox1 indicates the positions of respiratory supercomplexes $\left(\mathrm{III}_{2} \mathrm{IV}_{2}\right.$ and $\left.\mathrm{III}_{2} \mathrm{IV}\right)$ and complex IV. Dashed lines mark the small Rcf3 complexes, R3a and R3b. 


\subsection{Investigation of a potential interplay of Rcf proteins}

The surprising sequence similarity between $\operatorname{Rcf} 2^{\mathrm{N}}$ and $\operatorname{Rcf} 3$, as well as the interaction behavior described before, lead to the question of a functional overlap within the Rcf2 family. Given that Rcf2 ${ }^{\mathrm{N}}$ is highly unstable and probably rapidly degraded, a regulatory function, possibly within the respiratory chain, could be envisioned.

\subsubsection{Overexpression of Rcf2 fragments or Rcf 3 is harmless}

Based on this hypothesis, overexpression of either Rcf2 ${ }^{\mathrm{N}}$, or the related Rcf3, might disturb a balanced system and lead to negative effects on respiration. To test this possibility, Rcf2 fragments, as well as Rcf3, were expressed under control of their endogenous promoter, but from a multicopy plasmid (Figure 2-22). Figure 2-22 A illustrates that overexpression of Rcf3 does not cause a growth or respiratory phenotype, neither in wild-type nor in $r c f 3 \Delta$. Likewise, neither FLAGRcf2 ${ }^{\mathrm{N}}$ nor Rcf2C affected respiration in the conditions used in Figure 2-22 B. A FLAG-tagged version of Rcf2 ${ }^{\mathrm{N}}$ was used to enable detection of the fragment. The analysis of whole cell lysates in this case revealed an additional unexpected detail. The fragment generated in Figure 2-1, approximating the length of Rcf2C, is slightly larger than the one that is observed in mitochondria. Hence, the C-terminal fragment expressed in this experiment $\left(\mathrm{Rcf}^{\mathrm{C}-\mathrm{p}}\right)$ still contains the processing site and was therefore cleaved into the mature $\operatorname{Rcf} 2^{C}\left(\operatorname{Rcf} 2^{\mathrm{C}-\mathrm{m}}\right)$. 
A

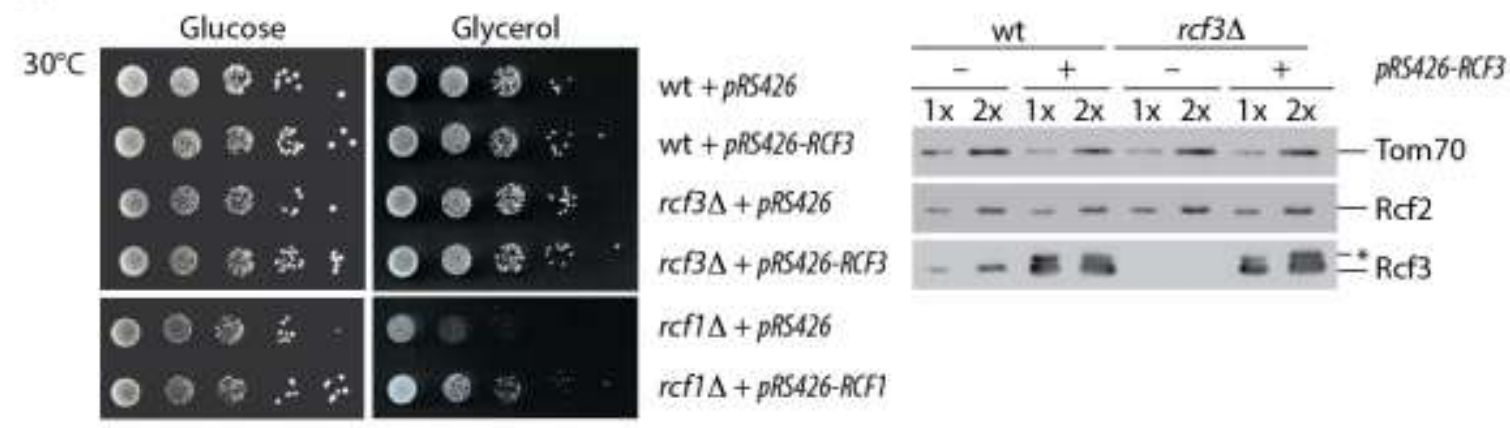

B

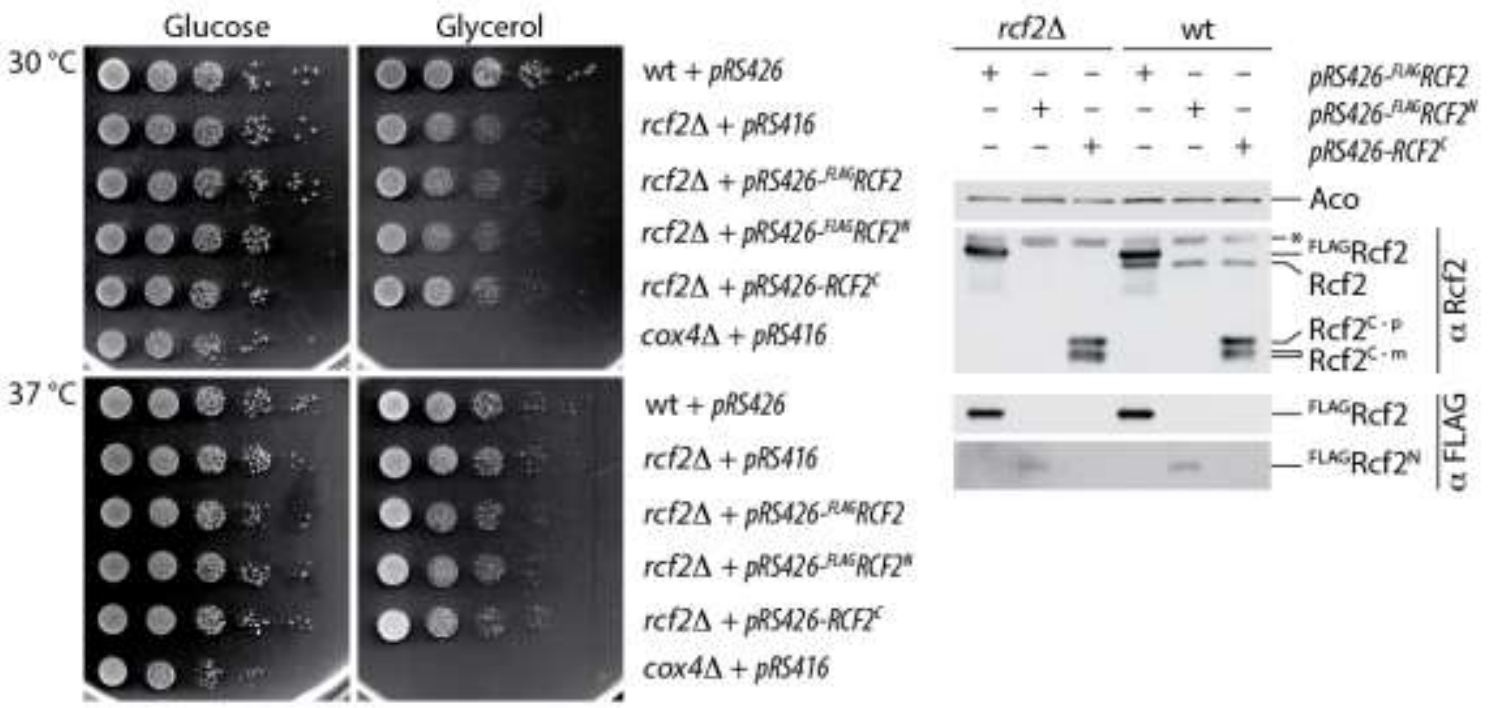

Figure 2-22: Respiration is not affected by overexpression of Rcf3, $\operatorname{Rcf}^{\mathrm{N}}$ or Rcf2C. A) Wild-type (wt) or $r c f 2 \Delta$ cells overexpressing Rcf3 were tested for growth on minimal media lacking uracil supplemented with glucose or glycerol at the indicated temperature. Growth was compared to wild-type and respiratory deficient $r c f 1 \Delta$. Isolated mitochondria from the same strains were subjected to SDS-PAGE and western blotting. The asterisk $\left(^{*}\right)$ marks a non-referable reaction of the

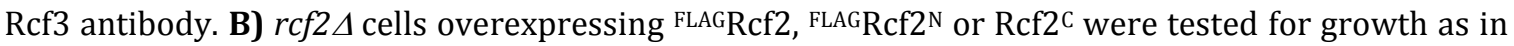
A. Growth was compared to wild-type and respiratory deficient $\operatorname{cox} 4 \Delta$. Whole cell lysates of wild-type (wt) and $r c f 2 \Delta$ cells overexpressing ${ }^{\text {FLAGRcf2, }}{ }^{\text {FLAGRcf }}{ }^{\mathrm{N}}$ or Rcf2 ${ }^{\mathrm{C}}$ were analyzed by SDS-PAGE and western blotting. The asterisk $\left({ }^{*}\right)$ marks a cross reaction of the Rcf2 antibody to an unrelated protein.

\subsection{2. $R c f 2^{\mathrm{N}}$ and $\operatorname{Rcf}^{\mathrm{C}}$ are not able to complement $\operatorname{rcf} 2 \Delta \operatorname{rcf} 3 \Delta$}

The overexpression did not point towards a regulatory function for $\operatorname{Rcf} 2^{\mathrm{N}}$, or a functional redundancy with Rcf3. Therefore, a means to test for the ability of different constructs to complement for a respiratory defect was needed. Since neither $R C F 2$, nor $R C F 3$, single deletion leads to a respiratory growth defect, a set of double and triple deletion mutants, representing all combinations of $R C F$ gene deletion, was created (Figure 2-23 A). The results of the growth tests seemed, at first glance, to be quite heterogeneous. However, upon biochemical analysis, they revealed a common characteristic. Deletion of $R C F 3$ in the $r c f 1 \Delta$ strain improved its 


\section{RESULTS}

ability to respire, albeit without visibly restoring the missing $\mathrm{III}_{2} \mathrm{IV}_{2}$ supercomplex (Figure 2-23 A, B and C). The same holds true, to some extent, for removing RCF3 from the severely affected $r c f 1 \Delta r c f 2 \Delta$. On the other hand, deletion of RCF3 in the unaffected $r c f 2 \Delta$ strain generated a growth defect similar to the one observed in rcf1 $\Delta$ (Figure 2-23 A and B). Strikingly, the only difference observed in BN-PAGE analysis of supercomplexes comprised of a slight increase in $\mathrm{III}_{2} \mathrm{IV}_{2}$ along with a slight decrease in free $\mathrm{III}_{2}$. Though the principles behind these data remain elusive, $r c f 2 \Delta r c f 3 \Delta$ has provided a possibility to test for functionality of the Rcf2 fragments.

A

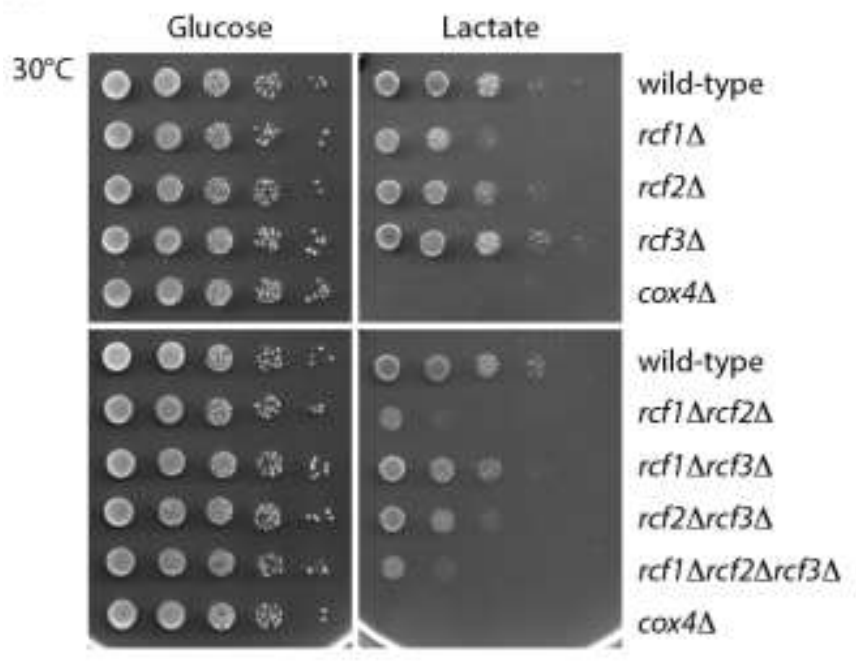

B

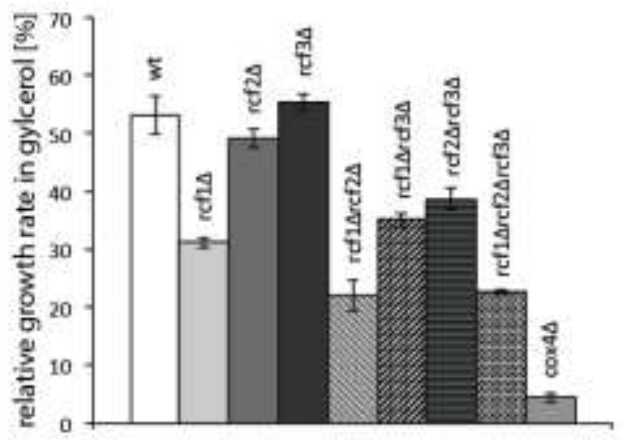

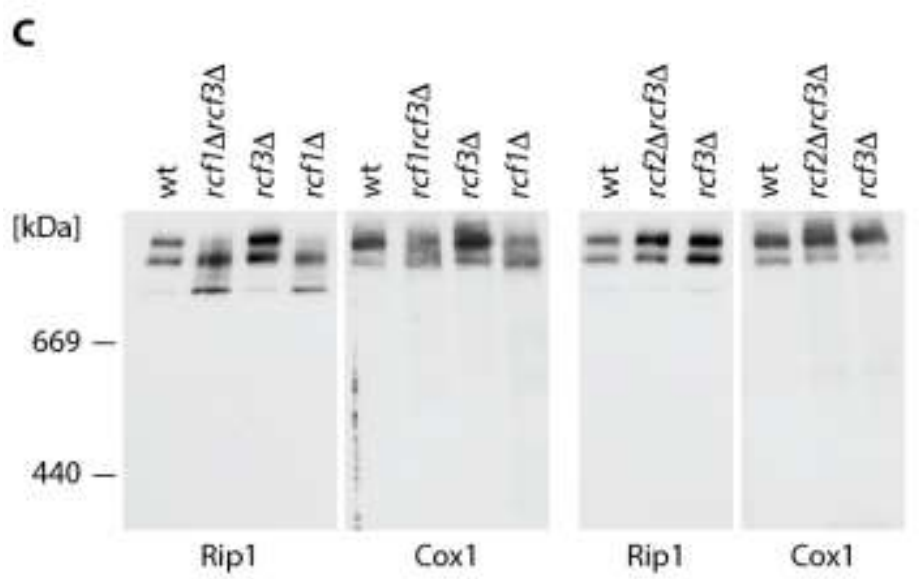

Figure 2-23: Double deletion of $R C F 2$ and $R C F 3$ generates a strain impaired in respiration. A) Indicated single, double and triple deletion strains of $R C F 1, R C F 2$ and $R C F 3$ were tested for growth on minimal media lacking uracil complemented with glucose or lactate at $30^{\circ} \mathrm{C}$. Growth was compared to wild-type and respiratory deficient $\operatorname{cox} 4 \Delta$. B) Strains indicated in A were used for a growth test in liquid YP supplemented with glucose or glycerol at $30^{\circ} \mathrm{C} . \mathrm{OD}_{595}$ was measured in regular intervals over a course of $12 \mathrm{~h}$. Glycerol growth rates were calculated relative to glucose (D) for each strain in quintuplicates and averaged (mean of $n=5 \pm$ STDEV). C) Isolated mitochondria of wild-type (wt) and indicated mutants were subjected to BN-PAGE and western blotting. 
The Rcf2 ${ }^{\mathrm{N}}$ fragment used before (Figure 2-22 B) contains an N-terminal FLAG tag. So far it had not been possible to fully exclude a deleterious effect of the tag itself on protein function. Thus, prior to the complementation test for $\operatorname{Rcf} 2^{\mathrm{N}}$ and $\operatorname{Rcf} 2^{\mathrm{C}}$, it was first tested whether FLAGRcf2 is able to substitute Rcf2 in $r c f 2 \Delta r c f 3 \Delta$. In order to mimic endogenous protein amounts, all constructs were expressed from a single copy plasmid under the control of their endogenous promoter. Figure 2-24 A illustrates that N-terminal tagging of Rcf2 interferes with its function independent of its expression level (compare expression of FLAGRcf2 from pRS416 and pRS426). For this reason, all $\mathrm{N}$-terminal tags were removed, accepting that the expression level of Rcf2 ${ }^{\mathrm{N}}$ will not be traceable anymore via western blotting. While both untagged Rcf2 and Rcf3 were able to restore growth in $r c f 2 \Delta r c f 3 \Delta$, neither Rcf2 ${ }^{\mathrm{N}}$ nor Rcf2C could complement the phenotype (Figure 2-24 B). This argues against a functional relevance of the processed fragments. However, a correlation with the incorrect length of the constructs used, especially Rcf2 ${ }^{\mathrm{N}}$, cannot be fully excluded.

A

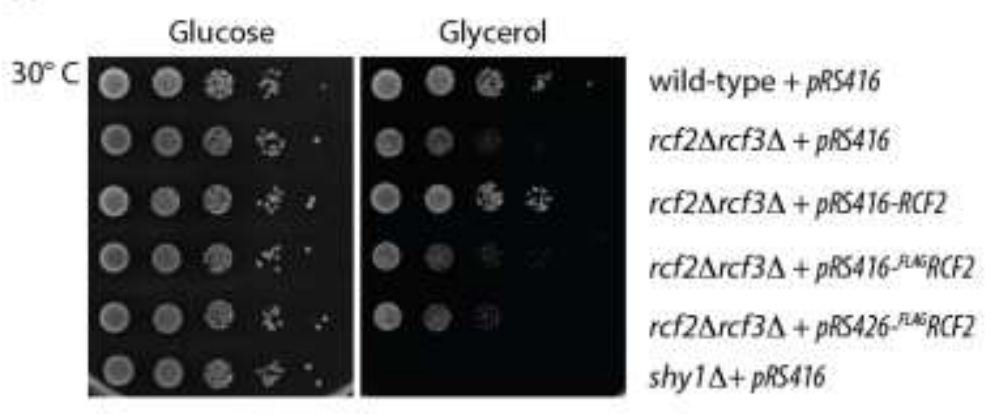

B

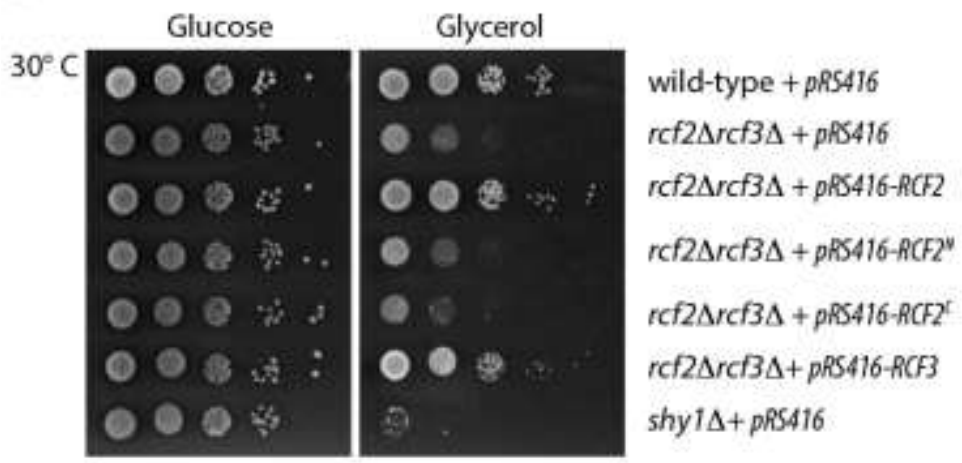

Figure 2-24: Untagged Rcf2 and Rcf3 can complement $r c f 2 \Delta r c f 3 \Delta$, while $\operatorname{Rcf}^{\mathrm{N}}$ and $\operatorname{Rcf}^{\mathrm{C}}$ are non-functional. A) $r c f 2 \Delta r c f 3 \Delta$ cells expressing Rcf2 or FLAGRcf2 at endogenous ( $p R S 416$ ) or increased ( $p R S 426$ ) levels were tested for growth on minimal media lacking uracil, complemented with glucose or glycerol at $30^{\circ} \mathrm{C}$. Growth was compared to wild-type and respiratory deficient shy1 $\Delta$. B) $r c f 2 \Delta r c f 3 \Delta$ cells expressing Rcf2, Rcf2 $2^{\mathrm{N}}, \mathrm{Rcf}^{\mathrm{C}}$ or Rcf3 were tested as in A. 


\subsubsection{Attempts to define specific Rcf2 interaction partners in vivo}

The respiratory deficient $r c f 2 \Delta r c f 3 \Delta$ also provided means to test the cysteine mutants that were used for the topology studies (Figure 2-3). The cysteine-free Rcf2 ${ }^{\mathrm{C} 705}$, as well as all mutants with introduced cysteines, were able to complement (Figure 2-25) and were thus considered to be functional.

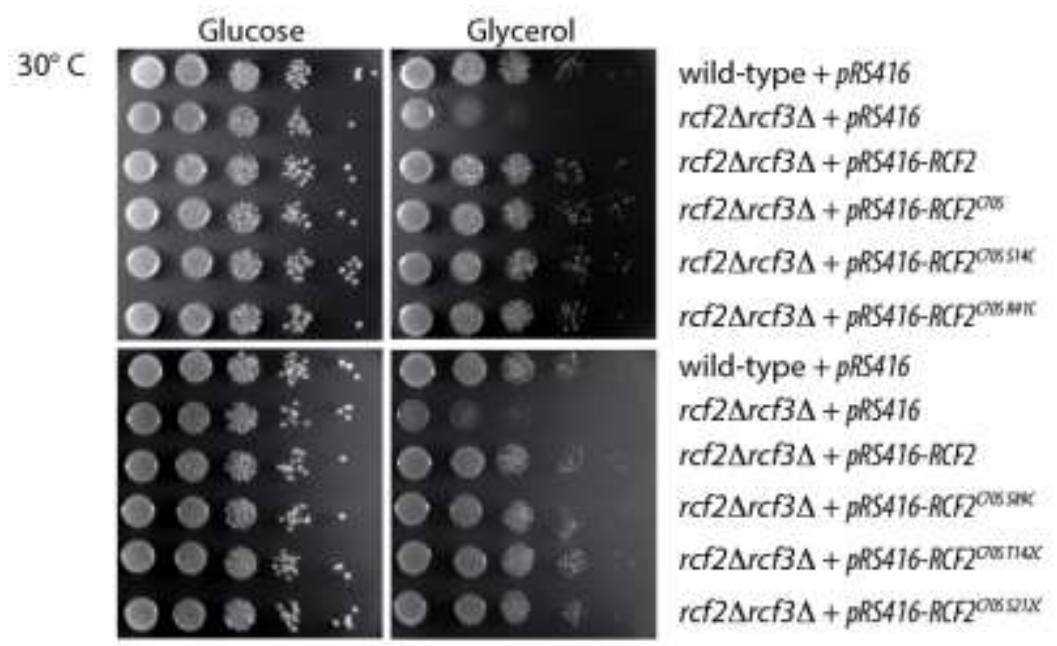

Figure 2-25: All cysteine mutant versions of Rcf2 are functional. $r c f 2 \Delta r c f 3 \Delta$ cells expressing Rcf2,

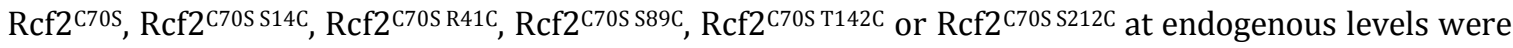
tested for growth on minimal media lacking uracil complemented with glucose or glycerol at $30^{\circ} \mathrm{C}$. Growth was compared to wild-type and respiratory impaired $r c f 2 \Delta r c f 3 \Delta$.

Copper has the ability to promote the formation of disulfide bridges between neighboring cysteine residues. The mutant strains thus provide a useful tool to determine Rcf2 interaction partners at specific positions in the proteins topology using a combined copper cross-linking and mass spectrometry approach. To this end, the different mutants were expressed in $r c f 2 \Delta$ in place of endogenous Rcf2 and subsequently subjected to $\mathrm{CuSO}_{4}$ treatment. Naturally, this cross-linking approach is limited by the requirement for a cysteine in the neighboring protein, in addition to the accessibility of both cysteines. Nevertheless, Figure 2-26 proved that it was indeed possible to cross-link interaction partners to Rcf2. When probed with the Rcf2 antibody, a specific pattern appeared upon the addition of $\mathrm{CuSO}_{4}$ that strongly differed depending on the position of the cysteine. 


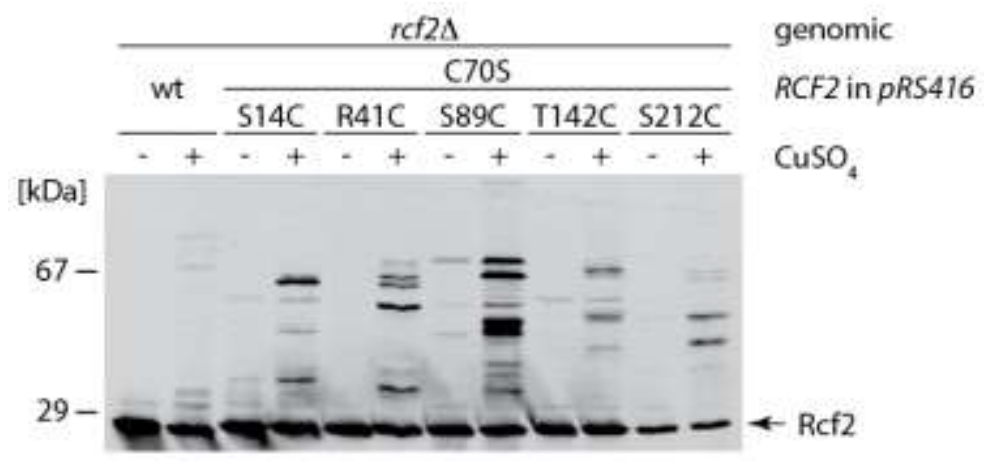

Figure 2-26: Insertion of cysteines at different positions leads to specific changes in the crosslinking pattern. Isolated mitochondria of $r c f 2 \Delta$ cells expressing wild-type Rcf2 or the indicated mutant versions were incubated with $\mathrm{CuSO}_{4}$. After quenching with NEM and EDTA, samples were analyzed by SDS-PAGE and western blotting, and probed for Rcf2.

However, prior to a mass spectrometric analysis it is imperative to isolate the observed cross-links in a clean and efficient way. Due to the antibody's high cross reactivity with unrelated proteins, the elutions from Rcf2 immunoprecipitations are unfit for this purpose. Therefore, a two-step purification procedure was evaluated, focusing on the endogenous cysteine in wild-type Rcf2 in a Cox4ZZ background. Since Rcf2 is part of complex IV, it is likely to closely interact with one of the other subunits. Among these, several contain a cysteine. Thus, after cross-linking, complex IV was isolated via Cox $4 \mathrm{ZZ}$ from DDM-solubilized mitochondria. The TEVcleaved elution provided pre-cleaned starting material for a subsequent denaturing Rcf2 immunoprecipitation in Triton X-100 (Figure 2-27 A). 


\section{RESULTS}

A

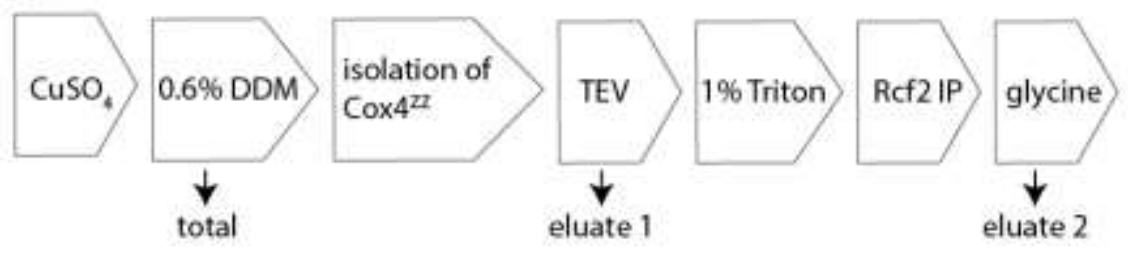

B

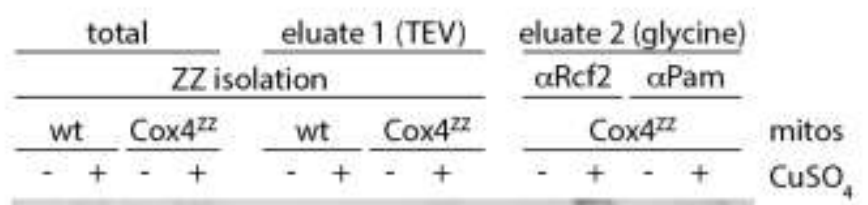

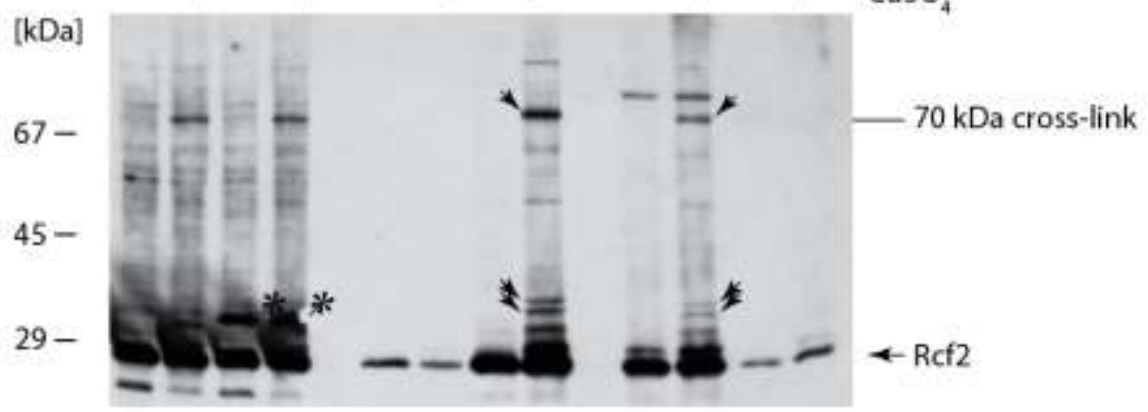

Figure 2-27: Two-step purification after copper cross-linking enriches a $70 \mathrm{kDa}$ cross-link in wild-type. A) Scheme for the cross-linking and isolation procedure used in B. B) Isolated mitochondria of $\mathrm{Cox}_{4 \mathrm{ZZ}}$ and wild-type (wt) were subjected to copper cross-linking, followed by isolation of Cox4 ${ }^{\mathrm{ZZ}}$ followed by immunoprecipitation of Rcf2 as indicated in A. Samples were analyzed by SDS-PAGE and western blotting (totals: 5\%; eluates. 100\%). The membrane was probed for Rcf2. Asterisks mark Cox 4 ZZ prior to TEV cleavage in the total lanes. Black arrows indicate prominent Rcf2 cross-links enriched in both eluates.

Figure 2-27 B demonstrates the enrichment of Rcf2 and Rcf2 cross-links after isolation of Cox4, suggesting that most of the interaction partners close to position C70 are situated within complex IV. After isolation of Rcf2, the signal intensity was decreased, most probably due to proteolytic events during the long isolation procedure. However the most prominent cross-link, with a size of approximately $70 \mathrm{kDa}$, remained detectable. 
Since the quality of the isolation was below the requirements for mass spectrometry, cross-linked samples were probed with all available antibodies against cysteinecontaining subunits of complex IV (Figure 2-28). As exemplified by immunodetection of the catalytic subunits, $\operatorname{Cox} 1, \operatorname{Cox} 2$ and $\operatorname{Cox} 3$, none of the tested proteins was responsible for the formation of the $70 \mathrm{kDa}$ band (data not shown for: Cox4, Cox6, Cox12, Cox13). Rcf1 and Rcf3 were also tested, but neither of them could be shown to interact with C70 using this approach (data not shown). A possibility that needs to be further tested is that Rcf2 potentially forms a dimer.

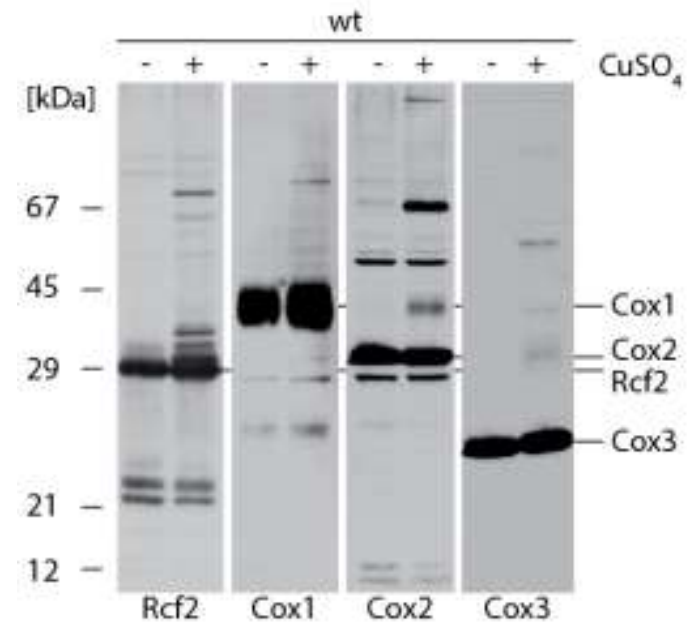

Figure 2-28: The $70 \mathrm{kDa}$ cross-link is unlikely to contain another subunit of complex IV. Isolated wild-type (wt) mitochondria were incubated with $\mathrm{CuSO}_{4}$. After quenching with NEM and EDTA, samples were analyzed by SDS-PAGE and western blotting. Membranes were probed as indicated. 


\section{Discussion}

Extensive research in the past decade has shown that complex III and complex IV of the yeast respiratory chain interact to form respiratory active supercomplexes $\left(\mathrm{III}_{2} \mathrm{IV}_{2}\right.$ and $\left.\mathrm{III}_{2} \mathrm{IV}\right)$. To date, several factors are known to influence supercomplex formation and stability. These range from lipids, via metabolite carriers, to specific protein factors such as the yeast protein Rcf1 (Böttinger et al., 2012; Chen et al., 2012; Dienhart \& Stuart, 2008; Strogolova et al., 2012; Vukotic et al., 2012; Zhang et al., 2002; 2005). While Rcf1 clearly proved its importance for the stability of $\mathrm{III}_{2} \mathrm{IV}_{2}$, the related Rcf2 seems to be dispensable for respiratory supercomplexes (Vukotic et al., 2012). The sequence alignment presented in this study (Figure 2-12) mapped the Rcf1 homology region of Rcf2 to its C-terminus. The Rcf2 N-terminus resembles Rcf3, an uncharacterized protein encoded by the open reading frame YBR255C-A. Since the regulation of supercomplex formation is still not understood, this study aimed for a characterization of the proposed Rcf protein family in regard to their impact on the respiratory chain complexes.

\subsection{Maturation of Rcf 2 comprises a proteolytic event}

\subsubsection{Limited proteolysis of Rcf2 by an unknown protease}

Rcf2 is a mitochondrial protein that associates with respiratory supercomplexes (Strogolova et al., 2012; Vukotic et al., 2012). However, a targeting signal in the form of a cleavable presequence had not been predicted. The protein had also not been found to be N-terminally truncated in a mitochondrial N-proteome analysis (Vögtle et al., 2009). Hence, in size, mature Rcf2 remains indistinguishable from the nonimported precursor protein (Vukotic et al., 2012). The present study reveals that mature Rcf2 is processed within mitochondria, although in an unusual way. During the processing event, up to $50 \%$ of Rcf2 is cleaved within the second TMD (section 2.1.1 and 2.1.2), resulting in at least one stable fragment, Rcf2 ${ }^{\mathrm{C}}$. Similarities between TMD2 of Rcf2 and the cleavage sites recognized by rhomboid proteases of different organisms (Strisovsky et al., 2009) led to the assumption that the mitochondrial rhomboid protease, Pcp1, might be responsible. Rhomboid proteases belong to the small group of proteases that are able to cleave a substrate within a 


\section{DISCUSSION}

membrane, rendering this hypothesis most appealing. Even though the topology and hence the intramembrane cleavage site of Rcf2 was confirmed in section 2.1.2, Pcp1 proved to be uninvolved (section 2.1.3). Via the cleavage of membrane-anchored proteins, rhomboids usually generate soluble active fragments (reviewed in Urban, 2006). Rcf2C, on the other hand, contains two TMDs and most likely remains in the membrane, thereby challenging the rhomboid hypothesis.

Further potential candidates are the $m$-AAA and $i$-AAA proteases of the inner membrane. These proteases mainly degrade non-native membrane-embedded polypeptides of the respiratory chain (reviewed in Koppen \& Langer, 2007). However, there is also evidence for the specific processing of proteins into alternative variants (Mrpl32) (Nolden et al., 2005). As it cannot be fully excluded that processing takes place prior to membrane insertion, in principle also other proteases might play a role. However, processing of Rcf2 took place in all mutants of mitochondrial proteases that were analyzed in a screen, among them also mutants for the $m$-AAA and $i$-AAA proteases (unpublished data; Nora Vögtle, Institute for Biochemistry and Molecular Biology, University of Freiburg).

Likely, other mitochondrial proteases exist that have not yet been identified, one of which might be the Rcf2 processing enzyme. Alternatively, two or more proteins working in concert might be necessary, as described for the cleavage of Ccp1 by the coordinated action of $m$-AAA and rhomboid (Esser, Tursun, Ingenhoven, Michaelis, \& Pratje, 2002; Tatsuta, Augustin, Nolden, Friedrichs, \& Langer, 2007). In this case, both proteases are essential for the event. Based on the data presented in section 2.1.3, Rcf2 processing could rather be mediated through several enzymes with redundant activities. It nevertheless remains remarkable that even the simultaneous inhibition of metallo-, serine-, cysteine- and aspartyl peptidases did not prevent Rcf2 cleavage. This aspect of the Rcf2 processing event therefore clearly needs further investigation.

\subsubsection{From regulation to degradation - the role of $\operatorname{Rcf}^{\mathrm{N}}$}

There are few examples in the literature for the removal of large N-terminal domains (ranging from 57 to 71 amino acids) from mitochondrial proteins. Such an event was described for the ribosomal proteins Mrpl38/34 (Kitakawa et al., 1997) and Mrpl32 (Nolden et al., 2005). Further examples are Mgm1 and Ccp1, which are 


\section{DISCUSSION}

cleaved by the mitochondrial rhomboid, Pcp1 (McQuibban et al., 2003). For neither of these processing events was a preservation of the N-terminal fragment reported. Also in the case of Rcf2, the role of Rcf2 ${ }^{\mathrm{N}}$ needs to be questioned due to the highly unstable nature of the fragment (section 2.1.4). Only an N-terminally FLAG-tagged Rcf2 construct enabled detection of $\operatorname{Rcf}^{\mathrm{N}}$ (section 2.1.1). However, further experiments using the $\mathrm{N}$-terminal tag later on uncovered a negative effect on Rcf2 function. Although the FLAG tag did not interfere with mitochondrial localization, processing or the comigration with complex IV of the full length protein (sections 2.1.1 and 2.1.4), it prevented complementation in the respiratory deficient $r c f 2 \Delta r c f 3 \Delta$ strain (section 2.3.2). Consequentially, a deleterious effect on the stability or the interactome of $\operatorname{Rcf} 2^{\mathrm{N}}$ cannot be excluded.

The small amounts of Rcf2 ${ }^{\mathrm{N}}$ that remained stable after digitonin solubilization were not bound in complexes, but suspected to be free protein (section 2.1.4). Given that this is not an effect of the FLAG tag, a role for Rcf $2^{N}$ in the respiratory chain is hard to envision. The fragment might still have a regulatory function but a more likely hypothesis is that the $\mathrm{N}$-terminal fragment is removed to liberate $\operatorname{Rcf}^{\mathrm{C}}$ and is subsequently degraded (Figure 3-1), as was suggested for Mrpl32 and Mgm1 (Herlan et al., 2003; McQuibban et al., 2003; Nolden et al., 2005; Zick et al., 2009). For degradation of $\operatorname{Rcf} 2^{\mathrm{N}}$, a combined action of $i$-AAA (extraction from the membrane) and oligopeptidases of the IMS (digestion of peptides) is possible (for review see Koppen \& Langer, 2007).

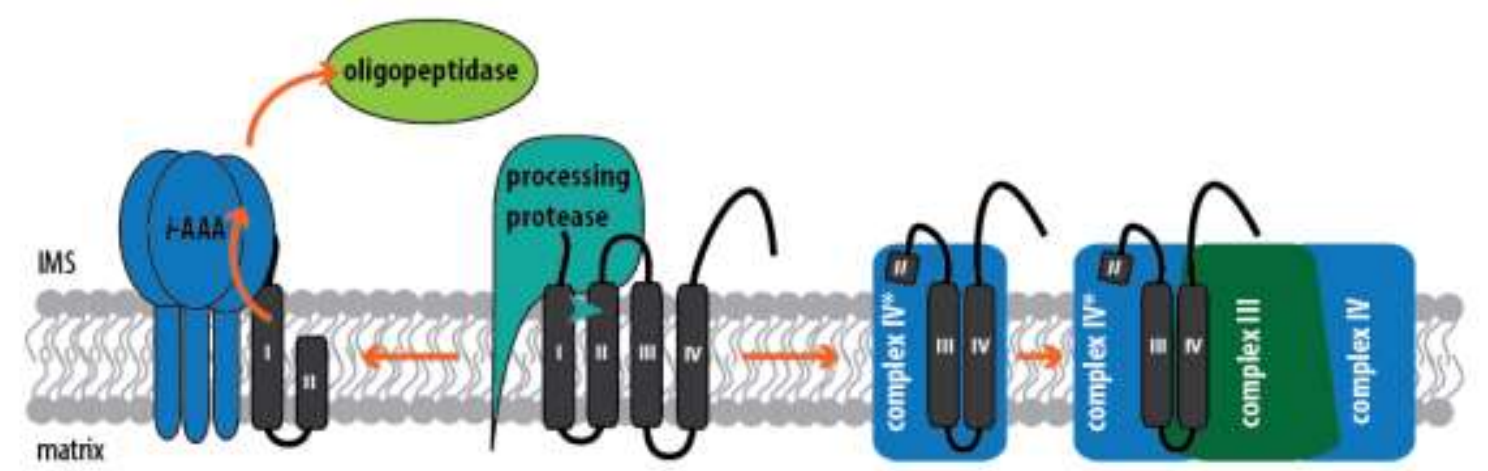

Figure 3-1: Cleavage of Rcf2 and possible fates of the resulting fragments, $\operatorname{Rcf} 2^{\mathrm{N}}$ and $\operatorname{Rcf}^{\mathrm{C}}$. After import and membrane insertion, a fraction of Rcf2 is cleaved by an undefined intramembrane processing protease within the second TMD. Rcf2C is then assembled into complex IV*, which is in turn used for the formation of supercomplexes. Rcf $2 \mathrm{~N}$ is most likely degraded, for example through combined action of the $i$-AAA and oligopeptidases of the IMS. 


\subsubsection{The functional relevance of Rcf2 processing - the role of $\operatorname{Rcf}^{\mathrm{C}} \mathrm{C}$}

In contrast to $\operatorname{Rcf} 2^{\mathrm{N}}, \operatorname{Rcf} 2^{\mathrm{C}}$ behaved more similar to the full length protein. IgG chromatography of Cox4 ${ }^{\mathrm{ZZ}}$, immunoprecipitation of C-terminally tagged Rcf2 and two-dimensional PAGE analysis demonstrated that $\mathrm{Rcf}^{\mathrm{C}}$ is a constituent of complex IV* and both respiratory supercomplexes (section 2.1.4). Hence, it is present in all identified Rcf2-containing complexes, except for the small Rcf2 complex of approximately $100 \mathrm{kDa}\left(\mathrm{R} 2^{100}\right)$. Small Rcf2-containing complexes had already been observed in the initial analysis, where they were explained as assembly intermediates (Vukotic et al., 2012). However, these conclusions were drawn from in vitro import and assembly studies, which are most likely sensitive enough to catch transient assembly intermediates. In the present study, R2 ${ }^{100}$ was detected at steady state and in large quantities. This strongly argues against this complex being an assembly intermediate, which is usually detected at low quantities. In many studies assembly intermediates were hence shown after blockage of the assembly path or isolation through specific assembly factors (Bareth et al., 2013; Gruschke et al., 2012; Mick et al., 2007).

Even though, $\operatorname{Rcf}^{\mathrm{C}}$ closely resembles full length Rcf2 in terms of supercomplex association, the two proteins seem to differ in function. The respiratory defect of the $r c f 2 \Delta r c f 3 \Delta$ strain is rescued by expression of $\operatorname{Rcf} 2$, but not $\mathrm{Rcf}^{\mathrm{C}}$ (section 2.3.2). It was not possible to assign a function to $\operatorname{Rcf} 2^{C}$ and hence, the purpose of the processing, as well as the timing, remains speculative. Import and assembly of Rcf2 truncations suggest that $\operatorname{Rcf}^{C}$ assembles into supercomplexes on its own (section 2.1.5), pointing towards a pre-assembly cleavage. However, since the truncation used as the $\operatorname{Rcf}^{C}$ (construct $C_{1}$ ) is slightly bigger than endogenous $\operatorname{Rcf}^{C}$ (section 2.3.2), it is still possible that $C_{1}$ is processed after assembly. To address this question, separation of the processed and unprocessed construct $C_{1}$ using a second dimension PAGE would be needed. However, a clear difference between Rcf2c-p and Rcf2 ${ }^{\mathrm{C}-\mathrm{m}}$, as seen for whole cell lysates in Figure 2-22, was not observed for the imported radiolabeled construct $C_{1}$. Hence the above-mentioned experiment needs further revision. Interestingly, every Rcf2 truncation harboring TMD3 and 4, and at least a stretch of the adjacent C-terminal IMS domain, was able to assemble into supercomplexes (section 2.1.5). Also the amino acids of the loop connecting TMD2 and 3 were dispensable (data not shown). Truncations starting with TMD3 do not 
contain the processing site. Their successful assembly hence further supports a preassembly processing event as shown in Figure 3-1.

A look into the processing of subunits of the respiratory chain and the mitochondrial ribosome confirms that pre-assembly processing is a relatively established principle. However, usually such proteins are completely transformed into the shorter and mature version. For instance, pre-Cox2 is matured by an inner membrane protease prior to its assembly into complex IV (Behrens, Michaelis, \& Pratje, 1991; Hell, Tzagoloff, Neupert, \& Stuart, 2000; Pratje, Mannhaupt, Michaelis, \& Beyreuther, 1983; A. Schneider et al., 1991) and Mrpl32 has to be processed by $m$-AAA in order to associate with the ribosome (Nolden et al., 2005). An example more similar to the limited proteolysis of Rcf2 though, is the processing of Mgm1, a member of the mitochondrial fusion machinery. In contrast to Rcf2, it contains a cleavable presequence that is removed by the mitochondrial processing peptidase, in order to generate the membrane-spanning Mgm1-l (Herlan et al., 2003). Afterwards however, the rhomboid protease, Pcp1, processes 50-95\% of Mgm1-l, liberating soluble but membrane-attached Mgm1-s. Mutation of the putative Pcp1 recognition site results in a non-cleavable Mgm1-1* (Herlan et al., 2003; McQuibban et al., 2003), which was used as a tool to prove the importance of processing for proper mitochondrial morphology (Herlan et al., 2003). These reports emphasize how a similar, non-cleavable, Rcf2 construct could contribute to a deeper understanding of the roles of Rcf2 and Rcf2C. Elimination of Rcf2 processing could have deleterious effects on respiration. In the case of Mgm1, it was shown that altering the ratio of the two forms towards Mgm1-l provokes a phenotype similar to the knockout (Zick et al., 2009). Due to the lack of a phenotype in $r c f 2 \Delta$, it will be necessary to undertake complementation assays with such a construct in the $r c f 2 \Delta r c f 3 \Delta$ background.

\subsection{Import of Rcf 2 might rely on TIM23 and OXA1}

Despite the carrier-like multi-spanning topology of Rcf2, this study clearly showed that the import of Rcf2 does not depend on the TIM22 machinery (section 2.1.6). Another possibility for imported membrane proteins to reach the inner mitochondrial membrane is the TIM23 machinery. Most substrates of this pathway contain a presequence and a single TMD, followed by a stop-transfer signal, that 


\section{DISCUSSION}

enables lateral release into the inner membrane (Botelho et al., 2011). The multispanning Rcf2 differs in both aspects. Nevertheless, there are examples of proteins with unusual targeting signals, such as C-terminal or internal targeting signals, that rely on the TIM23 machinery (C. M. Lee et al., 1999; Reinhold et al., 2012). However, a region serving as a targeting signal could not be identified for Rcf2 (section 2.1.5). Additionally, Rcf2 is partially imported in the absence of membrane potential, which is usually indispensible for TIM23-mediated import. Overall, Rcf2 therefore seems to be a rather poor substrate for TIM23. Accordingly, the results presented in section 2.1.6 do not argue for a clear-cut dependency on Tim23. Since the initial 30\% reduction in import was almost abolished after eight minutes (Figure 2-11), the involvement of further import mechanisms is conceivable.

A polytopic membrane protein can also be inserted by the coordinated action of the TIM23SORT complex and the OXA1 machinery, as was first shown for the ABC transporter Mdl1 (Bohnert et al., 2010). Distinct domains of this protein are inserted from different sites of the membrane by combining stop transfer and lateral release, with TIM23/PAM-mediated matrix import and conservative sorting via Oxa1 (Bohnert et al., 2010). The same mechanism has also recently been suggested for other mitochondrial transporters and subunits of the TIM22 machinery (Stiller et al., 2016).

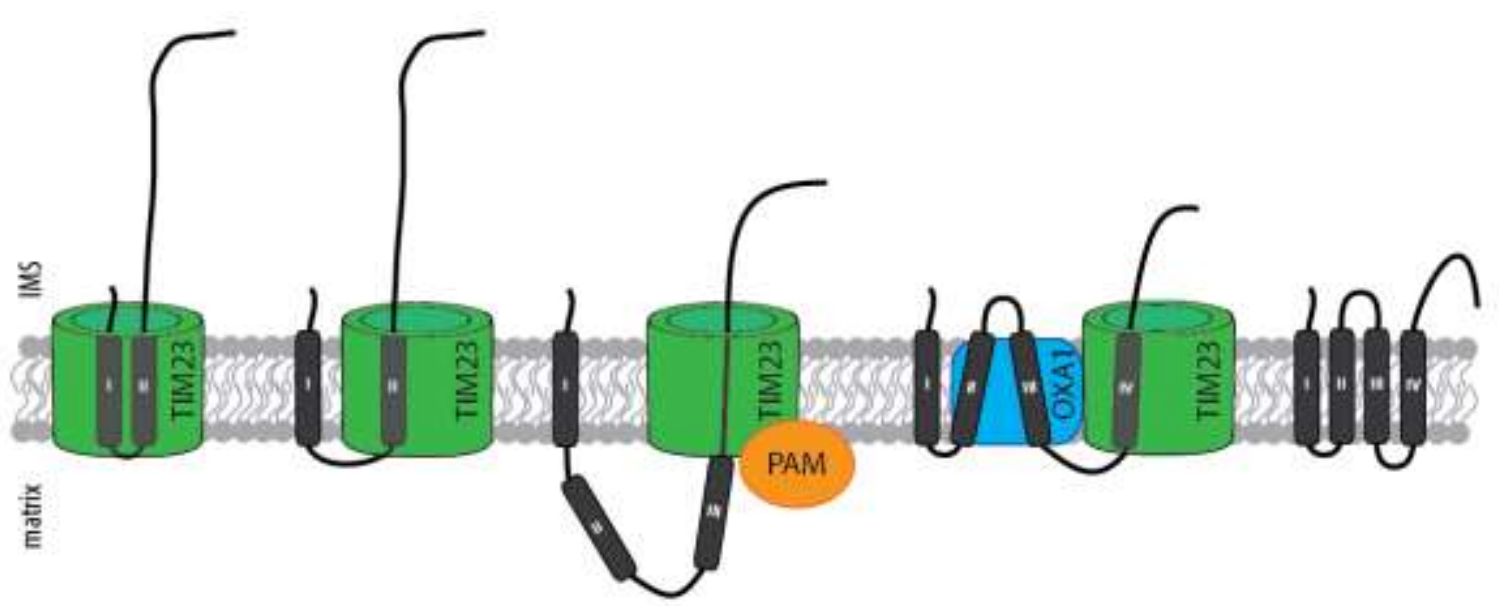

Figure 3-2: Hypothetical import pathway of Rcf2, through the combined action of TIM23 ${ }^{\text {soRT, }}$ TIM23/PAM and OXA1. The Rcf2 N-terminus, which emerges from the TOM pore, is inserted into TIM23 in a hairpin structure. This enables sorting and lateral release of TMD1 by TIM23 ${ }^{\text {SORT }}$ with the $\mathrm{N}$-terminus facing the IMS. TMD2 and 3 are translocated into the matrix, with the help of PAM, and are subsequently inserted into the inner membrane by Oxa1/Cox18. For an IMS located C-terminus, TMD4 is laterally released by TIM23 $3^{\text {SORT. }}$ 


\section{DISCUSSION}

TMDs arrested in the inner membrane by a stop transfer signal are usually more hydrophobic than those that are imported into the matrix (Botelho et al., 2011; Meier, Neupert, \& Herrmann, 2005; Park, Botelho, Hong, Österberg, \& Kim, 2013). Compared to TMDs 1 and 4, TMDs 2 and 3 of Rcf2 are less hydrophobic. Additionally, TMD2 contains proline, which also favors matrix translocation (Botelho et al., 2011; Meier et al., 2005). Based on this information, it can be speculated that Rcf2, might enter the TIM23 complex in a hairpin loop. After stop transfer and the release of TMD1 into the inner membrane, TMDs 2 and 3 could be imported in a PAM-dependent way, followed by insertion into the membrane via Oxa1/ Cox18. The last TMD would then be inserted similar to the first (Figure 3-2). In a screen for Oxa1-dependent nuclear encoded proteins, Stiller and coworkers indeed identified Rcf2, but did not biochemically confirm this finding (Stiller et al., 2016). Despite several facts speaking in favor of this hypothesis, some anomalies still remain, including the partial membrane potential and TIM23 independence. Even though evidence exists for other membrane potential-independent Tim23 substrates (Reinhold et al., 2012; Turakhiya et al., 2016), a careful biochemical analysis will be required to test the import model depicted in Figure 3-2.

\subsection{Rcf proteins reside at the interface of complex III and IV}

\subsubsection{Rcf3 is associated with, but not essential for, supercomplexes}

The analysis of Rcf3 (YBR255C-A) presented in section 2.2.1 confirmed its localization in the inner mitochondrial membrane and positioned the C-terminus in the IMS. Depending on the prediction algorithm, the number of TMDs varies from one to two. Since Rcf3 contains two cysteines, a maleimide PEG approach could be used to further investigate its topology. The TMD localization of these cysteines renders the approach problematic, as demonstrated for the endogenous cysteine in Rcf2 (section 2.1.2). Instead of a true second TMD, a hydrophobic and membrane associated patch could be envisioned. With this, Rcf3 would resemble Rcf2 ${ }^{\mathrm{N}}$ even more closely. During the cleavage event, the latter loses a part of its second TMD, which is then probably too short to span a membrane (Hildebrand, Preissner, \& Frömmel, 2004). 


\section{DISCUSSION}

In vitro import and assembly, IgG chromatography and immunoprecipitation experiments (sections 2.2.2 and 2.2.3) confirmed the supercomplex association of Rcf3 predicted by Helbig and coworkers (Helbig et al., 2009). Despite its presence in supercomplexes, Rcf3 is not essential for respiration or individual complex function (section 2.2.2). The same had already been observed for Rcf2 (Vukotic et al., 2012). In contrast to published data on Rcf1 and Rcf2 (Strogolova et al., 2012; Vukotic et al., 2012), supercomplex association of all three Rcfs might be mediated not only through complex IV, but also through complex III (sections 2.2.3 and 2.2.5). This would position Rcf proteins at the interface between the two complexes, which strongly supports a role in supercomplex organization. However, a causal connection with supercomplex formation or stability only exists for Rcf1. Inversely, not every protein with an impact on supercomplex assembly or stability necessarily interacts with complex III and complex IV in the same way. We have recently characterized Cox26 as a novel and exclusively complex IV-bound protein that influences respiratory supercomplexes (Levchenko et al., 2016). Of the four proteins discussed above, the most likely candidate for a true supercomplex-specific factor hence remains Rcf1. Based on this work and earlier studies, Rcf1 combines two characteristics assumingly important for such a factor: an influence on supercomplexes and an interaction with both complexes individually.

\subsubsection{How do Rcf proteins fit in the current crystal structures?}

Interestingly, none of the three Rcf proteins appear in the crystal structures available for respiratory complexes. However, it has to be noted that for yeast, only the structure of complex III is solved (Hunte et al., 2000). Nevertheless, crystallization has been performed for the bovine complex IV (Tsukihara et al., 1995), but for Rcf2 and Rcf3 a mammalian homologue is not predicted. The mammalian Rcf1a (or Higd1a) is described as a homologue for Rcf1. However, the structures were obtained after purification of single complexes in decyl maltoside (Tsukihara et al., 1995) or dodecyl maltoside (Hunte et al., 2000). Once supercomplex assemblies are disrupted by dodecyl maltoside treatment, all three Rcfs, especially Rcf3, are largely lost (section 2.2.3) (Vukotic et al., 2012). For supercomplexes on the other hand, a crystal structure does not exist. Available is a pseudo-atomic model of the $\mathrm{III}_{2} \mathrm{IV}_{2}$ supercomplex which was obtained by fitting the 
above described crystal structures into a cryo-EM three-dimensional map generated with digitonin-purified yeast complexes (Mileykovskaya et al., 2012). A protrusion facing the IMS between the cytochrome $c$ binding sites of complex III and IV was speculated to contain Rcf1 and Rcf2. Along the same lines, Hayashi and coworkers propose a Higd1a binding close to the catalytic center of mammalian complex IV (Hayashi et al., 2015). Both studies thus support the hypothesis that Rcf proteins localize to the complex III/IV interface.

\subsubsection{Complex IV* - reasons for an Rcf-specific version of complex IV}

Even though Rcf proteins also proved to associate with complex III, their main interaction partner in wild type mitochondria is complex IV, based on immunoprecipitations and IgG chromatography (sections 2.2 .3 and 2.2.5). In addition, second dimension PAGE analysis clearly showed comigration of all Rcfs, including Rcf2C, with the specific Rcf-containing version of complex IV (IV*). Astonishingly, in vitro import and assembly of Rcf3 and Rcf1 led to differently sized complexes, among which only the ones containing Rcf1 correlated with Cox1 staining (section 2.2.2). A similar discrepancy between in vitro assembly and in vivo steady state analysis was also observed for Rcf2. In vitro assembly of Rcf2 was completely abolished in the absence of Rcf1 (Vukotic et al., 2012), however large amounts of Rcf2 were detected in the remaining supercomplexes of $r c f 1 \Delta$ in this study (section 2.2.5). This had already been observed to lesser extents by Vukotic and coworkers, who explained it by a less efficient but still ongoing assembly of Rcf2 in vivo (Vukotic et al., 2012). Nevertheless, the question remains how the discrepancy between in vitro and in vivo data can be explained.

What furthermore needs to be addressed in the future is the biological relevance of the Rcf-specific complex IV*. Based on elevated levels of reactive oxygen species in $r c f 1 \Delta$ and $r c f 2 \Delta$, it had been suggested to protect the respiratory chain from ROS (Vukotic et al., 2012). Association of Rcf1 and Rcf2 during the late steps of assembly was furthermore proposed to prime complex IV for the formation of supercomplexes (Vukotic et al., 2012; Römpler et al., under revision). Other groups have reported an interaction between Rcf1 and Cox3 at earlier steps and hence a complex IV assembly phenotype is observed rather than a defect in supercomplex assembly (McStay, Su, \& Tzagoloff, 2013; Strogolova et al., 2012). The two 


\section{DISCUSSION}

perceptions do not necessarily have to oppose to each other. Rcf proteins proved to be present in several complexes (sections 2.2.4 and 2.2.5) and hence could interact at different steps of complex IV assembly. To date, the stoichiometry of all three proteins is not known, but it has been speculated that more than one molecule of Rcf1 could be engaged in tethering respiratory complexes (Mileykovskaya et al., 2012).

\subsection{Small Rcf complexes could serve as an interaction platform}

\subsubsection{The importance of the Rcf3 C-terminus for small Rcf3 complexes}

For all three Rcf proteins, second dimension PAGE analysis revealed the existence of several complexes considerably smaller than complex IV. Two Rcf3-containing complexes of unknown composition were defined in this study: R3a ( 140 kDa) and R3b ( $70 \mathrm{kDa})$. In fact, these two complexes bind the majority of Rcf3, while only a small fraction of the protein is assembled into supercomplexes in vivo (section 2.2.5). The observation that most Rcf3 was assembled into supercomplexes in vitro can be traced back to the usage of $r c f 3 \Delta$ mitochondria (section 2.2.2). Weak in vitro supercomplex assembly of Rcf3 in wild-type mitochondria points to a limited number of Rcf3 binding sites within supercomplexes, which are usually occupied in wild-type and preferentially filled up in $r c f 3 \Delta$. Furthermore, the C-terminus of Rcf3 seems to be crucial for maintaining the correct ratio of supercomplex and R3a/R3b distribution. The addition of a ZZ tag leads to an equal signal distribution in both pools (section 2.2.3), probably due to steric hindrance by the large protein A moiety. The efficiency of Rcf3 and Rcf3zz detection differs greatly. Hence, it is not possible to directly compare the overall protein amounts and to distinguish between taginduced increased supercomplex assembly and decreased stability of Rcf3zz in $\mathrm{R} 3 \mathrm{a} / \mathrm{R} 3 \mathrm{~b}$. The role of the C-terminus therefore needs further investigation.

\subsubsection{Assessing complex IV-independent Rcf-Rcf interactions}

Based on their comigration pattern (section 2.2.5), the small complexes, R3b and $\mathrm{R} 2^{100}$, could provide a platform for the complex IV-independent interaction of Rcf3 and Rcf2 observed using immunoprecipitation experiments (section 2.2.5). On the other hand, Rcf3 coisolated considerable amounts of Rcf1, which is present in a 


\section{DISCUSSION}

small complex that does not comigrate with that of Rcf3. Along the same lines, neither R3b nor R2 ${ }^{100}$ change their running behavior or intensity in the absence of the other respective Rcf. This implies that the interaction takes place in another context. The observed decrease of supercomplex-bound Rcf3 in $r c f 1 \Delta$ is most likely based on the overall reduction of $\mathrm{III}_{2} \mathrm{IV}_{2}$, which is the major Rcf3-containing supercomplex. In the case of disrupted supercomplex assembly, an accumulation of complex IV-bound protein would be anticipated. Instead, Rcf3 seems to accumulate in the smaller R3a complex (section 2.2.5). Data provided in this study illustrates that the association of R3a, or small Rcf complexes in general, with complex IV or complex III assembly is unlikely. Hence, the formation of an interaction platform for Rcf, and potentially other proteins, remains the most appealing hypothesis for the function of small Rcf complexes. It cannot be excluded that the conditions in which protein complexes are exposed during BN-PAGE disrupted larger assemblies (Schägger, 2001) into the smaller modules visible in the second dimension PAGE analysis. If this is the case, important impacts of single deletions on complex organization might have been missed.

Copper-induced disulfide bond formation, used as an alternative approach to detect interactions between Rcf2 and Rcf1, Rcf3 or other proteins, revealed that Rcf2 might interact with itself. This is especially interesting not only in light of the suggested functionally relevant interaction of Mgm1-l with Mgm1-s (Rujiviphat et al., 2015; Zick et al., 2009), but also regarding the potential involvement of Rcf2 in tethering respiratory complexes to each other. A similar function had been proposed for Tim11/Atp21, a complex V subunit that was observed to dimerize and thus mediate complex V dimer formation (Arnold, Pfeiffer, Neupert, Stuart, \& Schägger, 1998; Arselin et al., 2003; Brunner, Everard-Gigot, \& Stuart, 2002). To thoroughly test whether Rcf2 self interacts, the strategy presented in this study needs to be adjusted in a way that enables discrimination between two different Rcf2 versions. To omit the use of tags, import of an Rcf2 construct lacking the antibody epitope, followed by copper cross-linking and immunoprecipitation of endogenous Rcf2, will be one suitable approach. 


\section{DISCUSSION}

\subsubsection{Connecting Rcf2 and Rcf3 - the split paralogue hypothesis}

The homology of Rcf1 and Rcf3 with different parts of Rcf2 prompted the speculation that they might be split paralogues of Rcf2. In light of the Rcf2 processing, a functional overlap of Rcf3 with Rcf2 ${ }^{\mathrm{N}}$ was hypothesized. Double deletion of RCF2 and RCF3 did not influence the structure of respiratory complexes, but it did affect respiration. Hence, Rcf3 and Rcf2 ${ }^{\mathrm{N}}$ might have a common function in regulating respiratory complexes. In contrast to the model proposed in Figure 3-1, the rapid degradation of $\operatorname{Rcf} 2^{\mathrm{N}}$ could also be ascribed to a tight regulation of the fragment, leading to a high turn over rate.

In the context of a functional redundancy, complementation of the $\operatorname{rcf} 2 \Delta r c f 3 \Delta$ by Rcf2 ${ }^{\mathrm{N}}$ was anticipated, but not observed (section 2.3.2). As discussed in section 3.1.3, the $\mathrm{N}$-terminal fragment expressed in $r c f 2 \Delta r c f 3 \Delta$ is shortened by a few amino acids compared to the endogenous Rcf2 ${ }^{\mathrm{N}}$. This truncation might interfere with correct functioning of the construct. Therefore, a conclusion cannot be drawn prior to a repetition of the complementation studies using an adapted Rcf2 ${ }^{\mathrm{N}}$ construct. 


\section{Summary and Conclusion}

As the site of action of the OXPOS system, the mitochondrial network is the main supplier of ATP in the eukaryotic cell. Intensive research on the multisubunit complexes of the respiratory chain has revealed the existence of catalytically active and functionally advantageous respiratory supercomplexes. Even though their importance has been widely accepted by now, it is still under debate as to how respiratory supercomplexes are established and regulated. The "solid model", proposing permanent supercomplex organization of respiratory complexes, cannot fully account for several aspects of electron transport within the ETC. Hence an alternative model was formulated, suggesting dynamic changes between supercomplex and single complex state, depending on the cells energy demand and environment. Over the past decade, several protein and non-protein factors, among them Rcf1, were described to promote the formation or stability of supercomplexes, however, the precise mechanisms still remain to be elucidated.

This study aimed for a deeper understanding of supercomplex regulation by characterizing a group of related respiratory chain factors (Rcfs). We were able to show that Rcf3, the protein product of $Y B R 255 C$ - $A$, associates with supercomplexes via its interaction with complex IV*, as was previously described for Rcf1 and Rcf2. Like Rcf2, Rcf3 is not essential for respiration and the formation of supercomplexes. Rcf1 thus remains the only single protein factor described so far that influences supercomplex formation in yeast. Nevertheless, all three proteins associate with complex III in the absence of complex IV, suggesting that all Rcfs are more than exclusive complex IV subunits. Focusing on their ability to interact with either complex, it is tempting to envision them as the "proteinaceous glue" that holds supercomplexes together. Hence, an important task for the future will be to define the differences that render Rcf1 more important than Rcf2 and Rcf3.

Detailed analysis of Rcf2 revealed that this multispanning membrane protein presumably reaches the inner membrane through an unusual import pathway. The presented data and recent publications prompted the speculation that Rcf2 is imported and inserted into the membrane by a combination of lateral release from TIM23 and conservative sorting. It was furthermore shown that a part of Rcf2 is processed after import, but presumably prior to assembly, by a protease that is yet 


\section{CONCLUDING REMARKS}

to be identified. $\operatorname{Rcf} 2^{\mathrm{N}}$ and $\operatorname{Rcf} 2^{\mathrm{C}}$, the products of the cleavage, resemble Rcf3 and Rcf1 respectively. Based on protein alignments, Rcf1 and Rcf3 could be split paralogues of $\operatorname{Rcf} 2$, with a potential functional redundancy to $\operatorname{Rcf} 2^{C}$ and $\operatorname{Rcf} 2 \mathrm{~N}$. This study clearly showed that $\operatorname{Rcf} 2^{C}$ alone is not sufficient for proper Rcf2 functioning. However, whether increased levels of $\operatorname{Rcf} 2^{C}$ can overcome loss of Rcf1 is something to be tested in the future.

Due to technical issues, a role for $\operatorname{Rcf} 2^{\mathrm{N}}$ cannot be assigned based on the data presented here. Whether or not Rcf2 ${ }^{\mathrm{N}}$ is preserved, assembled into a complex or used as a supercomplex regulator will be revealed by further investigations. This will also help to determine the relevance of the processing event in general.

In summary, this work has provided an initial insight into the function and relevance of the novel Rcf protein family in respiratory chain complex assembly and organization. 


\section{Material and Methods}

\subsection{Materials}

\subsubsection{Kit systems, enzymes and reagents}

Standard chemicals in analytical grade were purchased from AppliChem (Darmstadt, Germany), Merck (Darmstadt, Germany), Roth (Karlsruhe, Germany), Serva (Heidelberg, Germany), or Sigma Aldrich (Taufkirchen, Germany). DNA primers were synthesized by Metabion (Martinsried, Germany) and Microsynth (Lindau, Germany). Commercial kit systems as listed in Table 5-1 were used according to the manufacturers instruction. Special chemicals and enzymes used in this study are listed in Table 5-2.

Table 5-1: Kit systems and enzymes used in this study

\section{Kit system Supplier}

Fast Digest restriction enzymes

Gene Ruler DNA Ladder $1 \mathrm{~kb}$

KOD Hot Start DNA Polymerase

mMESSAGE mMACHINE ${ }^{\circledR}$ SP6

Transcription Kit

Precision Plus Protein ${ }^{\mathrm{TM}}$ All Blue

Prestained Protein Standards 10-250 kDa

QuikChange Lightning

Site-Directed Mutagenesis Kit

Rapid Ligation Kit

TNT Flexi Translation

TNT Quick Coupled Transcription/

Translation SP6

Unstained SDS PAGE Protein Marker

6.5-200 kDa

Wizard ${ }^{\circledR}$ Plus SV Minipreps DNA

Purification System

Wizard ${ }^{\circledR}$ SV Gel and PCR Clean-Up System
Thermo Fisher Scientific

Thermo Fisher Scientific

Merck Millipore

Ambion ${ }^{\circledR} /$ Thermo Fisher Scientific

BioRad (München, Germany)

Agilent Technologies (Santa Clara

CA, USA)

Thermo Fisher Scientific

Promega

Promega

Serva

Promega

Promega 
Table 5-2: Special reagents and enzymes used in this study

\begin{tabular}{|c|c|}
\hline Reagent & Supplier \\
\hline [35S]-L-Methionine & Hartmann Analytic (Braunschweig) \\
\hline Acrylamide, $4 \mathrm{x}$ crystallized & Roth \\
\hline Agarose NEEO ultra quality & Roth \\
\hline ANTI-FLAG M2 affinity gel & Sigma Aldrich \\
\hline Antimycin A & Sigma Aldrich \\
\hline ATP & Roche \\
\hline CNBr activated sepharose $4 \mathrm{~B}$ & GE Healthcare \\
\hline cOmplete, EDTA free protease inhibitor & Roche \\
\hline Creatine kinase & Roche \\
\hline Creatine phosphate & Roche \\
\hline $\mathrm{CuSO}_{4} \cdot 5 \mathrm{H}_{2} \mathrm{O}$ & Merck \\
\hline Cytochrome $c$ from bovine heart & Sigma Aldrich \\
\hline Digitonin & Calbiochem ${ }^{\circledR} /$ Merck Millipore \\
\hline Dimethyl pimelimidate dihydrochloride & Sigma Aldrich \\
\hline Ethidiumbromide $0.07 \%$ & AppliChem \\
\hline FLAG $^{\circledR}$ peptide & Sigma Aldrich \\
\hline G418 sulphate (Genitincin) & PAA/ GE Healthcare \\
\hline Hering Sperm DNA & Promega \\
\hline IgG from human serum & Sigma Aldrich \\
\hline IgG protein standard & BioRad \\
\hline Immobilion ${ }^{\circledR}$-P PVDF membrane & Merck Millipore \\
\hline Medix XBU Medical X-ray film & Foma Bohemia (Hradec Králové, CZ) \\
\hline n-Dodecyl $\beta$-D-maltoside (DDM) & Sigma Aldrich \\
\hline $\begin{array}{l}\mathrm{N}, \mathrm{N}^{\prime} \text {-Methylene-bisacrylamide, } \\
2 \mathrm{x} \text { crystallized }\end{array}$ & Serva \\
\hline $\mathrm{NADH}$ & Roche \\
\hline $\mathrm{Ni}^{2+}$-NTA agarose & Quiagen \\
\hline Oligomycin & Sigma Aldrich \\
\hline PEG maleimid, average $M_{n} 2000$ & Sigma Aldrich \\
\hline Pierce $®$ ECL Western blotting substrate & Thermo Fisher Scientific \\
\hline Protein-A sepharose & GE Healthcare \\
\hline Proteinase K, recombinant & Roche \\
\hline Roti $^{\circledR}$-Quant & Roth \\
\hline Rotiphorese $^{\circledR}$ Gel 30 (37.5:1) & Roth \\
\hline Streptomycin sulfate salt & Sigma Aldrich \\
\hline Valinomycin & Calbiochem $^{\circledR} /$ Merck Millipore \\
\hline Zymolyase-20T & $\begin{array}{l}\text { Seikagaku Biobusiness Corporation } \\
\text { (Tokyo, Japan) and Nacalai Tesque } \\
\text { Inc. (Kyoto, Japan) }\end{array}$ \\
\hline
\end{tabular}




\subsubsection{Antibodies}

Polyclonal antibodies were raised in rabbit (Gramsch Laboratories, Schwabhausen, Germany) against C-terminal peptides, recombinant whole protein or recombinant protein domains. All commercially available monoclonal antibodies and probes used in this study are listed in Table 5-3. Antibodies and probes were diluted in Tris buffered saline with $0.1 \%$ Tween 20 (TBS-T) containing 5\% powdered milk or $1 \%$ bovine serum albumin (BSA). Secondary goat antibodies directed against rabbit IgG were used in a dilution of 1:20,000 (HRP) or 1:10,000 (fluorescent dye). Those directed against mouse IgG were used in a dilution of 1:3,000 (HRP) or 1:10,000 (fluorescent dye).

Table 5-3: Commercially available antibodies used in this study

\begin{tabular}{ll} 
Antibody & Supplier \\
\hline \hline$\alpha$ FLAG & Sigma Aldrich \\
Peroxidase Anti-Peroxidase Soluble Complex Antibody & Sigma Aldrich \\
Goat $\alpha$ Rabbit HRP & Dianova \\
Goat $\alpha$ Mouse HRP & Dianova \\
Goat $\alpha$ Rabbit DyLight 488 & Dianova \\
Goat $\alpha$ Mouse DyLight 488 & Dianova
\end{tabular}

\subsubsection{Plasmids}

All plasmids used or generated in this study are listed in Table 5-4. Plasmids were propagated in E. Coli XL1 Blue. 
MATERIAL AND METHODS

\subsubsection{Microorganisms}

Escherichia coli XL1 Blue (recA1 endA1 gyrA96 thi-1 hsdR17 supE44 relA1 lac [F'proAB lacIqZAM15 Tn10 (Tetr $\left.\left.{ }^{\mathrm{r}}\right)\right]$ was obtained by Stratagene and used for cloning. The majority of Saccharomyces cerevisiae strains used in this study is based on the YPH499 background. Exceptions are Rcf3GFP, pcp1A (BY4741), cox1- (777-3A) and cbp1 $\Delta$ (XPM-77). The wild-type strains and their derivatives are listed in Table 5-5. Not listed are control strains transformed with the empty plasmids pRS416 (URA3) or pRS426 (URA3). This was done for YPH499, $r c f 1 \Delta, r c f 2 \Delta, r c f 3 \Delta, r c f 2 \Delta r c f 3 \Delta$, shy1 $\Delta$ and $\operatorname{cox} 4 \Delta$. These strains were used as $U R A^{+}$controls for various mutants. 
Table 5-4: Plasmids used in this study

\begin{tabular}{|c|c|c|c|c|}
\hline Plasmid name & Purpose & Features & Marker & Reference \\
\hline pFA6aHIS3MX6 & PCR template for gene deletion by HIS3 & & HIS3 Amp & Longtine et al., 1998 \\
\hline pFA6aKANMX6 & PCR template for gene deletion by KAN & & $K A N$ Amp & Longtine et al., 1998 \\
\hline pFA6aNATNT1 & PCR template for gene deletion by NAT & & NAT Amp & Janke et al., 2004 \\
\hline pFA6aTRP1 & PCR template for gene deletion by TRP1 & & TRP1 Amp & Longtine et al., 1998 \\
\hline pRS416 & yeast plasmid & CEN & URA3 Amp & Sikorski \& Hieter, 1989 \\
\hline pRS426 & yeast plasmid & $2 \mu$ & URA3 Amp & $\begin{array}{l}\text { Christianson et al., } \\
1992\end{array}$ \\
\hline pYM10 & $\begin{array}{l}\text { PCR template for C-terminal TEV-ProtA-His7 } \\
\text { tagging }\end{array}$ & TEV-ProtA-His7 (ZZ) & HIS3 Amp & Knop et al., 1999 \\
\hline pYM2.2 & PCR template for C-terminal Strep-Flag tagging & Strep-Flag (SF) & HIS3 Amp & Janke et al., 2004 \\
\hline Rcf1个 (pKRB2) & overexpression of Rcf1 & $\begin{array}{l}R C F 1 \text { orf }+ \text { promoter } \\
\text { in pRS426 }\end{array}$ & URA3 Amp & this study \\
\hline FLAGRcf2个(pKRB15) & $\begin{array}{l}\text { overexpression of N-terminally FLAG-tagged } \\
\text { Rcf2 }\end{array}$ & ${ }^{F L A G} R C F 2$ in pRS426 & URA3 Amp & this study \\
\hline FLAGRcf2N $\uparrow(p K R B 16)$ & $\begin{array}{l}\text { overexpression of N-terminally FLAG-tagged } \\
\text { Rcf2 }{ }^{N}\end{array}$ & $F L A G R C F 2^{1-210}$ in pRS426 & URA3 Amp & this study \\
\hline Rcf2c个(pKRB17) & overexpression of $\operatorname{Rcf} 2^{C}$ & $R C F 2^{211-696}$ in pRS426 & URA3 Amp & this study \\
\hline FLAGRcf2 (pKRB5) & expression of N-terminally FLAG-tagged Rcf2 & FLAGRCF2 in pRS416 & URA3 Amp & Römpler et al. (rev.) \\
\hline Rcf2 (pKRB14) & expression of Rcf2 & $\begin{array}{l}R C F 2 \text { orf }+ \text { promoter } \\
\text { in pRS } 416\end{array}$ & URA3 Amp & this study \\
\hline $\operatorname{Rcf}^{N}{ }^{N}(p K R B 19)$ & expression of Rcf2 ${ }^{N}$ & $R C F 2^{1-210}$ in pRS416 & URA3 Amp & this study \\
\hline
\end{tabular}


MATERIAL AND METHODS

\begin{tabular}{|c|c|c|c|c|}
\hline Plasmid name & Purpose & Features & Marker & Reference \\
\hline $\operatorname{Rcf2C}^{C}(\mathrm{pKRB} 18)$ & expression of Rcf2C & $\begin{array}{l}\text { RCF2 } 211-696 \\
\text { in pRS416 }\end{array}$ & URA3 Amp & this study \\
\hline Rcf2 ${ }^{\mathrm{C} 70 \mathrm{~S}}$ (pKRB7) & expression of cysteine-free Rcf2 C70s & $R C F 2{ }^{\mathrm{C} 70 \mathrm{~S}}$ in $\mathrm{pRS} 416$ & URA3 Amp & this study \\
\hline Rcf2 C70S R41C (pKRB10) & expression of Rcf2 C70S R41C & 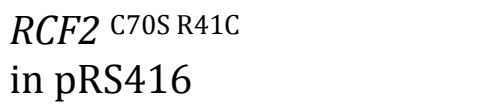 & URA3 Amp & this study \\
\hline Rcf2 C70s S14C (pKRB9) & expression of Rcf2 C70S S14C & $\begin{array}{l}R C F 2 \text { C70S S14C } \\
\text { in pRS416 }\end{array}$ & URA3 Amp & this study \\
\hline Rcf2 $\operatorname{c70S~S212C~(pKRB13)~}$ & expression of Rcf2 C70s S212C & $\begin{array}{l}\text { RCF2 } \mathrm{C} 70 \mathrm{~S} \mathrm{~S} 212 \mathrm{C} \\
\text { in pRS416 }\end{array}$ & URA3 Amp & this study \\
\hline Rcf2 $\operatorname{c70s~s89C}(p K R B 11)$ & expression of Rcf2 C70S S89C & $\begin{array}{l}R C F 2 \text { C70S S89C } \\
\text { in pRS416 }\end{array}$ & URA3 Amp & this study \\
\hline $\operatorname{Rcf2}^{\mathrm{C} 70 \mathrm{~S} \text { T142C (pKRB12) }}$ & expression of Rcf2 C70S T142C & $\begin{array}{l}\text { RCF2 } \mathrm{C} 70 \mathrm{~S} \mathrm{~T} 142 \mathrm{C} \\
\text { in pRS416 }\end{array}$ & URA3 Amp & this study \\
\hline Rcf3个 (pKRB4) & overexpression of Rcf3 & $\begin{array}{l}R C F 3 \text { orf }+ \text { promoter } \\
\text { in pRS426 }\end{array}$ & URA3 Amp & this study \\
\hline AEF1 & in vitro transcription/ translation of Oxa1 & $\begin{array}{l}\text { OXA1 orf + SP } 6 \text { promoter } \\
\text { in pCR-Blunt II-TOPO }\end{array}$ & Kan & Frazier et al., 2003 \\
\hline A01 & $\begin{array}{l}\text { in vitro transcription/ translation of } \\
\text { Neurospora crassa AAC }\end{array}$ & $\begin{array}{l}n c A A C \text { cDNA }+ \text { SP6 } \\
\text { promoter in pGEM } 4 \mathrm{Z}\end{array}$ & Amp & Pfanner et al., 1987 \\
\hline
\end{tabular}


Table 5-5: Yeast strains used in this study

\begin{tabular}{|c|}
\hline Yeast strain \\
\hline YPH499 \\
\hline BY4742 \\
\hline$r c f 1 \Delta(\mathrm{yMaD} 2)$ \\
\hline $\operatorname{rcf2} \Delta$ (MVY2) \\
\hline $\operatorname{rcf3} \Delta(\mathrm{KRY} 1)$ \\
\hline $\operatorname{cox} 4 \Delta(\mathrm{AFY} 11)$ \\
\hline cyt1 $\Delta$ (AFY10) \\
\hline $\operatorname{cox} 5 a \Delta(\mathrm{MVY} 15)$ \\
\hline shy1d \\
\hline pсp1 $1 \Delta$ \\
\hline $\operatorname{tim} 10-2$ \\
\hline $\operatorname{tim} 23 \Delta(\mathrm{MB} 29)$ \\
\hline pGAL-S Tim23 (yCS4) \\
\hline Cox4ZZ (HCY04) \\
\hline Cor1ZZ (MVY9) \\
\hline Rcf3 ${ }^{\mathrm{GFP}}$ (LJY66) \\
\hline Rcf3ZZ (KRY2) \\
\hline Rcf3 $^{S F}$ (KRY3) \\
\hline
\end{tabular}

\section{Genotype}

MATa ade2-101 his3-4200 leu2-41 ura3-52 trp1-463 lys2-801

MATa his3- $\Delta 1$ leu2 $\Delta 0$ met15 $\Delta 0$ ura3 $\Delta 0$

MATa ade2-101 his3-4200 leu2-41 ura3-52 trp1-463 lys2-801 YML030w::loxP MATa ade2-101 his3-4200 leu2-41 ura3-52 trp1-463 lys2-801 YNR018w::HIS3MX6

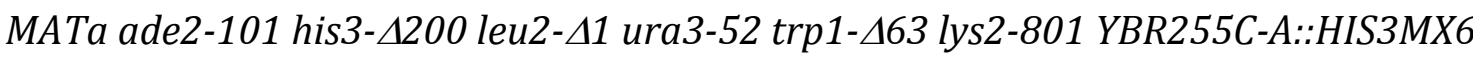

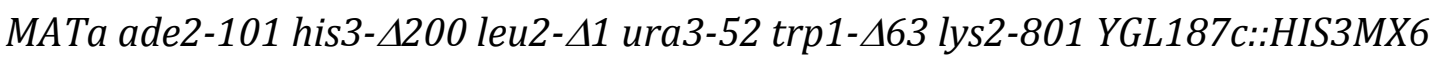
MATa ade2-101 his3-4200 leu2-41 ura3-52 trp1- 463 lys2-801 YOR065w::HIS3MX6

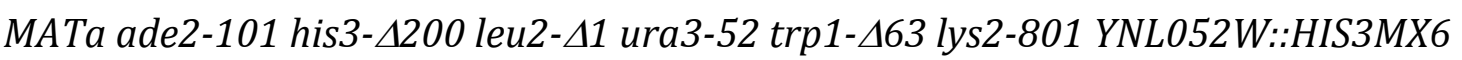

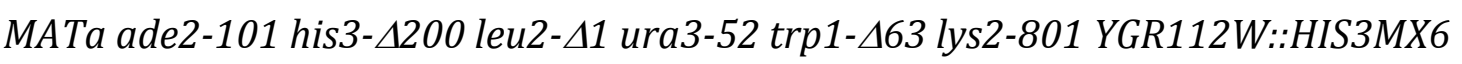
MATa his3-41 leu2 $\Delta 0$ met15 $\triangle 0$ ura3 $\triangle 0$ YGR101W::kanMX4

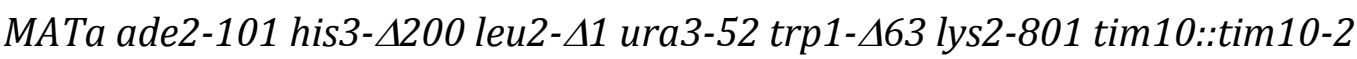

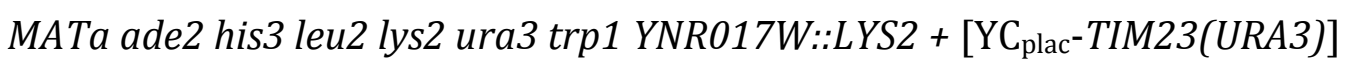
MATa ade2 his3 leu2 lys2 ura3 trp1 YNR017W::LYS2 + [pGAL-S-TIM23(HIS3)] MATa ade2-101 his3-4200 leu2-41 ura3-52 trp1-463 lys2-801 cox4::cox4-ZZ-HIS3MX6

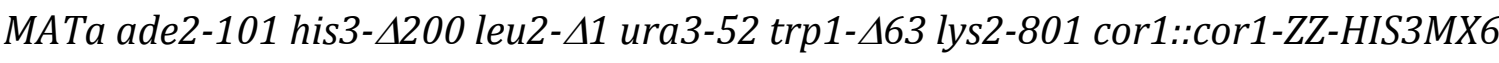
MATa his3- 11 leu2 $\Delta 0$ met15 $\Delta 0$ ura3 $\Delta 0$ rcf3::rcf3-EGFP-kanMX4

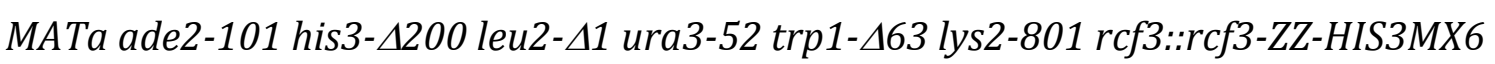

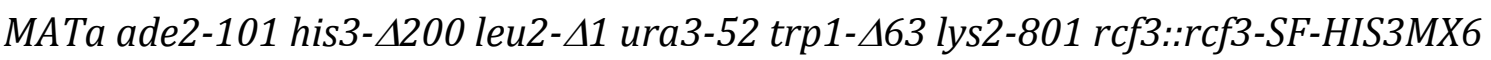

\section{Reference}

Sikorski \& Hieter 1989

Euroscarf

Vukotic et al., 2012 Vukotic et al., 2012 Römpler et al. (rev.) Frazier et al., 2006 Vukotic et al., 2012 Römpler et al. (rev.) Reinhold et al., 2012 Euroscarf

Truscott et al., 2002 Bömer et al., 1997 Schulz et al., 2011 Vukotic et al., 2012 Vukotic et al., 2012 Römpler et al. (rev.) this study Römpler et al. (rev.) 


\begin{tabular}{|c|}
\hline Yeast strain \\
\hline$r c f 1 \Delta r c f 2 \Delta$ \\
\hline$r c f 2 \Delta r c f 3 \Delta(\mathrm{KRY} 8)$ \\
\hline $\begin{array}{l}r c f 1 \Delta r c f 2 \Delta r c f 3 \Delta \\
\text { (KRY9a) }\end{array}$ \\
\hline$r c f 1 \Delta r c f 3 \Delta(\mathrm{KRY} 10 \mathrm{a})$ \\
\hline rho $^{0}(\mathrm{KRY} 11)$ \\
\hline$r c f 3 \Delta \mathrm{rho}^{0}(\mathrm{KRY} 12)$ \\
\hline wt FlagRcf2 (KRY27) \\
\hline $\begin{array}{l}\text { wt Rcf3个 } \\
(\mathrm{KRY} 21)\end{array}$ \\
\hline $\begin{array}{l}r c f 1 \Delta \operatorname{Rcf} 1 \uparrow \\
(\mathrm{KRY} 20)\end{array}$ \\
\hline $\begin{array}{l}r c f 3 \Delta \operatorname{Rcf} 3 \uparrow \\
(\mathrm{KRY} 24)\end{array}$ \\
\hline $\begin{array}{l}r c f 2 \Delta^{\text {FlagRcf2 }} \uparrow \\
(\mathrm{KRY} 40)\end{array}$ \\
\hline $\begin{array}{l}r c f 2 \Delta^{\text {FlagRcf2 }^{\mathrm{N}} \uparrow} \\
(\mathrm{KRY} 41)\end{array}$ \\
\hline $\begin{array}{l}r c f 2 \Delta \mathrm{Rcf} 2 \mathrm{C} \uparrow \\
\text { (KRY42) }\end{array}$ \\
\hline
\end{tabular}

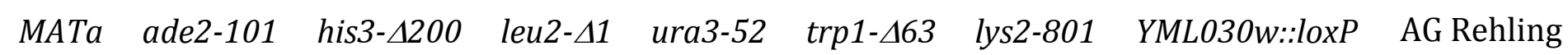
YNR018w::HIS3MX6

MATa ade2-101 his3-4200 leu2-41 ura3-52 trp1- 463 lys2-801 YNR018w::kanMX6 this study YBR255C-A::HIS3MX6

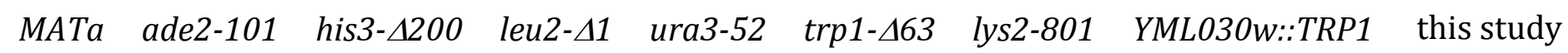
YNR018w::KANMX6 YBR255C-A::HIS3MX6

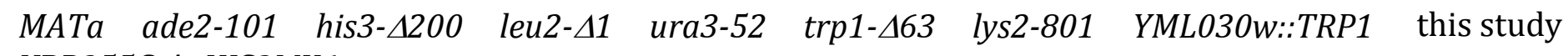
YBR255C-A::HIS3MX6

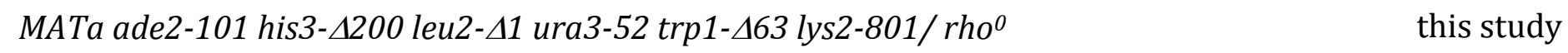

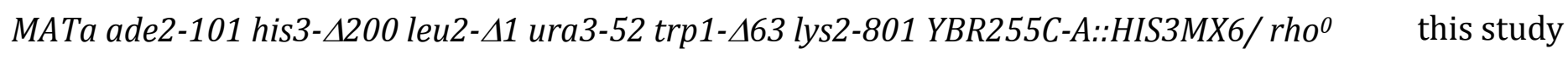

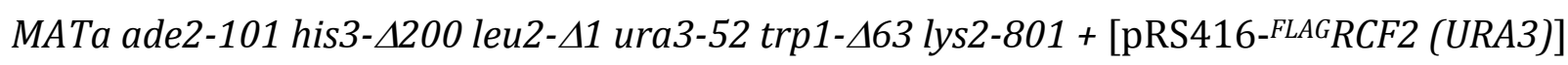
MATa ade2-101 his3-4200 leu2-41 ura3-52 trp1- 463 lys2-801 + [pRS426-RCF3 (URA3)]

MATa ade2-101 his3-4200 leu2-41 ura3-52 trp1-463 lys2-801 YML030w::loxP Römpler et al. (rev.) this study + [pRS426-RCF1 (URA3)]

MATa ade2-101 his3-4200 leu2-41 ura3-52 trp1-463 lys2-801 YBR255C-A::HIS3MX6 this study + [pRS426-RCF3 (URA3)]

MATa ade2-101 his3-4200 leu2-41 ura3-52 trp1-463 lys2-801 YNR018w::HIS3MX6 this study + [pRS426- ${ }^{-} L A G R C F 2$ (URA3)]

MATa ade2-101 his3-4200 leu2-41 ura3-52 trp1-463 lys2-801 YNR018w::HIS3MX6 this study $+\left[\mathrm{pRS} 426-{ }^{-} L A G R C F 2^{N}(U R A 3)\right]$

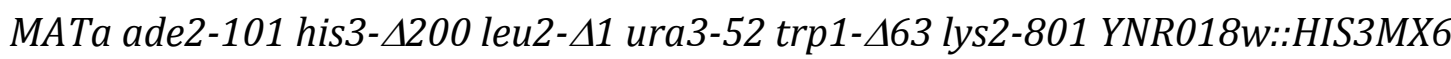

$+\left[\mathrm{pRS} 426-\mathrm{RCF}^{C}\right.$ (URA3)]

this study 


\begin{tabular}{|c|c|c|}
\hline Yeast strain & Genotype & Reference \\
\hline $\begin{array}{l}r c f 2 \Delta^{\text {FlagRcf2 }} \\
\text { (KRY29) }\end{array}$ & $\begin{array}{l}\text { MATa ade2-101 his3- } \triangle 200 \text { leu2- } \triangle 1 \text { ura3-52 trp1- } 463 \text { lys2-801 YNR018w::HIS3MX6 } \\
+ \text { [pRS416-FLAGRCF2 (URA3)] }\end{array}$ & Römpler \\
\hline $\begin{array}{l}r c f 2 \Delta \mathrm{Rcf} 2 \\
(\mathrm{KRY} 32)\end{array}$ & $\begin{array}{l}\text { MATa ade2-101 his3- } \triangle 200 \text { leu2- } \Delta 1 \text { ura3-52 trp1- } 463 \text { lys2-801 YNR018w::HIS3MX6 } \\
+ \text { [pRS416-RCF2 (URA3)] }\end{array}$ & this study \\
\hline $\begin{array}{l}\operatorname{rcf2} 2 \Delta \mathrm{Rcf} 2 \mathrm{C70S} \mathrm{S} 14 \mathrm{C} \\
\text { (KRY35) }\end{array}$ & 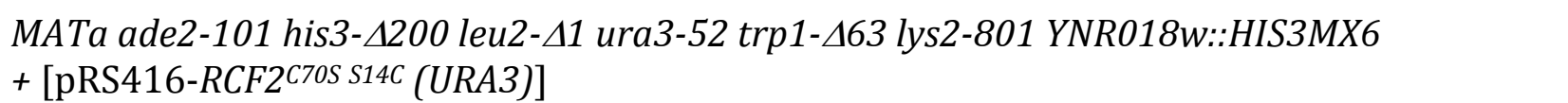 & this study \\
\hline $\begin{array}{l}r c f 2 \Delta \mathrm{Rcf}^{\mathrm{C}} \mathrm{C} 0 \mathrm{~S} \mathrm{R} 41 \mathrm{C} \\
(\mathrm{KRY} 36)\end{array}$ & $\begin{array}{l}\text { MATa ade2-101 his3- } \triangle 200 \text { leu2- } \Delta 1 \text { ura3-52 trp1- } \triangle 63 \text { lys2-801 YNR018w::HIS3MX6 } \\
+ \text { [pRS416-RCF2C70S R41C (URA3)] }\end{array}$ & this study \\
\hline $\begin{array}{l}\text { rcf2 } 2 \Delta \mathrm{Rcf} 2^{\mathrm{C} 70 \mathrm{~S} S 89 \mathrm{C}} \\
\text { (KRY37) }\end{array}$ & 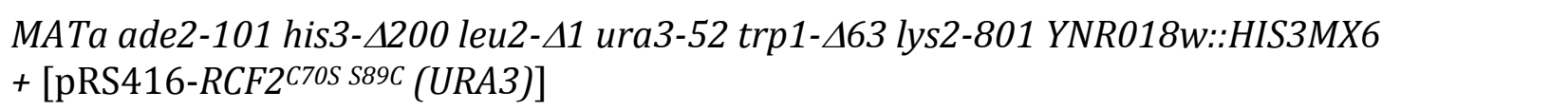 & this study \\
\hline $\begin{array}{l}r c f 2 \Delta \mathrm{Rcf} 2 \mathrm{C} 70 \mathrm{~S} \mathrm{~T} 142 \mathrm{C} \\
(\mathrm{KRY} 38)\end{array}$ & $\begin{array}{l}\text { MATa ade2-101 his3- } \triangle 200 \text { leu2- } \Delta 1 \text { ura3-52 trp1- } \triangle 63 \text { lys2-801 YNR018w::HIS3MX6 } \\
+ \text { [pRS416-RCF2C70s T142C (URA3)] }\end{array}$ & this \\
\hline $\begin{array}{l}r c f 2 \Delta \mathrm{Rcf} 2 \mathrm{C70S} \mathrm{S} 212 \mathrm{C} \\
(\mathrm{KRY} 39)\end{array}$ & $\begin{array}{l}\text { MATa ade2-101 his3- } \triangle 200 \text { leu2- } \Delta 1 \text { ura3-52 trp1- } \triangle 63 \text { lys2-801 YNR018w::HIS3MX6 } \\
+ \text { [pRS416-RCF2C70s S212C (URA3)] }\end{array}$ & this study \\
\hline $\begin{array}{l}r c f 2 \Delta r c f 3 \Delta \mathrm{Rcf} 2 \\
(\mathrm{KRY} 71)\end{array}$ & $\begin{array}{l}\text { MATa ade2-101 his3- } \triangle 200 \text { leu2- } \Delta 1 \text { ura3-52 trp1- } \Delta 63 \text { lys2-801 YNR018w::HIS3MX6 } \\
\text { YBR255C-A::HIS3MX6 + [pRS416-RCF2 (URA3)] }\end{array}$ & this study \\
\hline $\begin{array}{l}r c f 2 \Delta r c f 3 \Delta \text { FlagRcf2 } \\
\text { (KRY72) }\end{array}$ & $\begin{array}{l}\text { MATa ade2-101 his3- } \triangle 200 \text { leu2- } \triangle 1 \text { ura3-52 trp1- } \triangle 63 \text { lys2-801 YNR018w::HIS3MX6 } \\
\text { YBR255C-A::HIS3MX6 + [pRS416-FLAGRCF2 (URA3)] }\end{array}$ & this study \\
\hline $\begin{array}{l}r c f 2 \Delta r c f 3 \Delta \text { FlagRcf2个 } \\
\text { (KRY67) }\end{array}$ & 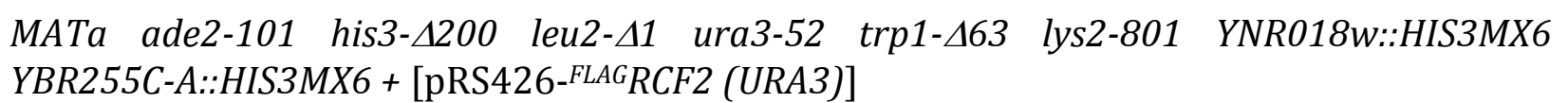 & this study \\
\hline $\begin{array}{l}r c f 2 \Delta r c f 3 \Delta \operatorname{Rcf} 2^{\mathrm{N}} \\
(\mathrm{KRY} 73)\end{array}$ & $\begin{array}{l}\text { MATa ade2-101 his3- } 4200 \text { leu2- } \Delta 1 \text { ura3-52 trp1- } 463 \text { lys2-801 YNR018w::HIS3MX6 } \\
\text { YBR255C-A::HIS3MX6 + [pRS416-RCF2N (URA3)] }\end{array}$ & this study \\
\hline
\end{tabular}


Yeast strain

$r c f 2 \Delta r c f 3 \operatorname{Rcf}^{C}$

(KRY74)

$r c f 2 \Delta r c f 3 \Delta$ Rcf3

(KRY75)

$r c f 2 \Delta r c f 3 \Delta$

Rcf2 ${ }^{\mathrm{C} 70 \mathrm{~S}}$ (KRY76)

$r c f 2 \Delta r c f 3 \Delta$

Rcf2 ${ }^{\mathrm{C} 70 \mathrm{~S} S 14 \mathrm{C}}$ (KRY77)

$r c f 2 \Delta r c f 3 \Delta$

Rcf2C70S T44C (KRY78)

$r c f 2 \Delta r c f 3 \Delta$

Rcf2C70S 589C (KRY79)

rcf2 $\Delta r c f 3 \Delta$

Rcf2 ${ }^{\mathrm{C} 70 \mathrm{~S}}$ T142C $(\mathrm{KRY} 80)$

$r c f 2 \Delta r c f 3 \Delta$

Rcf2 ${ }^{\text {C70S S212C (KRY81) }}$

\section{Genotype}

MATa ade2-101 his3-4200 leu2-41 ura3-52 trp1-463 lys2-801 YNR018w::HIS3MX6 YBR255C-A::HIS3MX6 + [pRS416-RCF2C (URA3)]

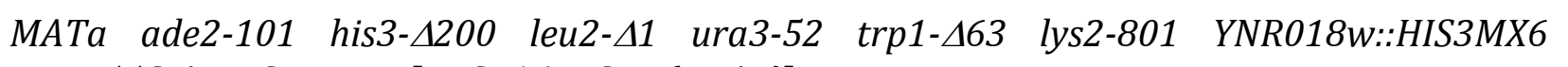
YBR255C-A::HIS3MX6 + [pRS416-RCF3 (URA3)]

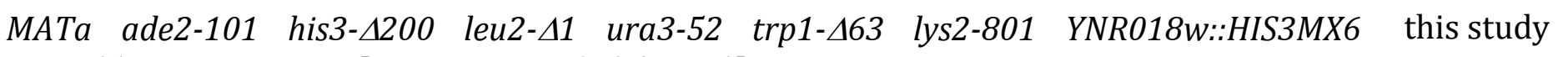
YBR255C-A::HIS3MX6 + [pRS416-RCF2C70S (URA3)]

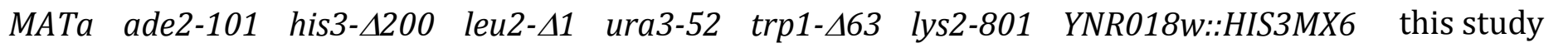
YBR255C-A::HIS3MX6 + [pRS416-RCF2 $\left.{ }^{C 70 S S 14 C}(U R A 3)\right]$

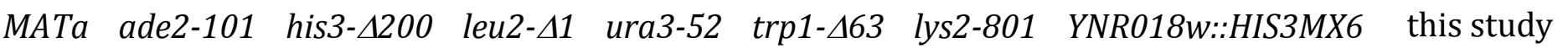
YBR255C-A::HIS3MX6 + [pRS416-RCF2C70S T44C (URA3)]

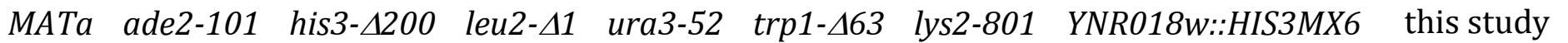
YBR255C-A::HIS3MX6 + [pRS416-RCF2C70S S89C (URA3)]

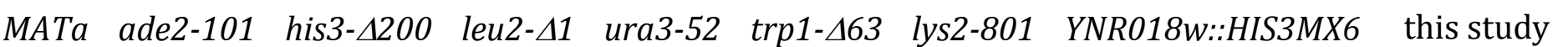

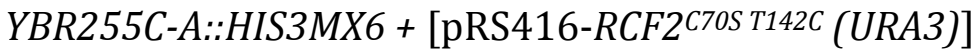

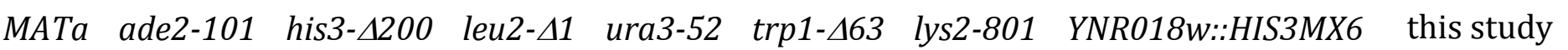
YBR255C-A::HIS3MX6 + [pRS416-RCF2C7OS S212C (URA3)]

\section{Reference}

this study

this study 


\subsubsection{Instruments and Software}

Instruments and Software used in this study are listed in Table 5-6 and Table 5-7.

Table 5-6: Instruments used in this study

\section{Instrument}

5417 R (centrifuge)

5424 (centrifuge)

$5804 \mathrm{R}$ (centrifuge)

Avanti J-26 XP (centrifuge)

Curix 60 (developing machine)

Fluorescence scanner FLA-9000

iMark $^{\mathrm{TM}}$ (microplate reader)

JA-10 (rotor)

JA-20 (rotor)

NanoVue Plus Spectrophotometer

Optima ${ }^{\text {TM }}$ L-90K (ultracentrifuge)

Optima ${ }^{\mathrm{TM}}$ MAX-XP (ultracentrifuge)

Potter S (dounce homogenisator)

Sorvall H-12000 (rotor)

Sorvall RC 12BP (centrifuge)

Speed Vac Concentrator

Storage Phosphor screens

Storm $^{\mathrm{TM}} 820$ PhosphorImager

Thermomixer comfort

TLA-55 (rotor)

TPersonal (thermo cycler)

UV Solo (UV documentation)

Vacuum gel drier

Varian Cary ${ }^{\circledR}$ Bio UV-Visible Spectrophotometer

Oxygraph $2 \mathrm{k}$

\section{Manufacturer}

Eppendorf

Eppendorf

Eppendorf

Beckman Coulter

AGFA

Fujifilm

BioRad

Beckman Coulter

Beckman Coulter

GE Healthcare

Beckman Coulter

Beckman Coulter

Sartorius

Thermo Fisher Scientific

Thermo Fisher Scientific

Savant

GE Healthcare

GE Healthcare

Eppendorf

Beckman Coulter

Biometra

Biometra

Scie-Plas

Agilent Technologies

Oroboros

Table 5-7: Software used in this study

Software

Geneious 5.3.6

Illustrator CS6

ImageQuant TL

Microsoft Office 2011

Papers ${ }^{3}$

Photoshop CS6

\section{Producer}

Biomatters (Auckland, New Zealand)

Adobe Systems (San Jose CA, USA)

GE Healthcare BioSciences AB (Uppsala, Sweden)

Microsoft Corporation (Redmond, USA)

Mekentosj (Aalsmeer, Netherlands)

Adobe Systems (San Jose CA, USA) 


\subsection{Cultivation and handling of microorganisms}

\subsubsection{Growth conditions for yeast}

Unless noted otherwise, yeast were grown according to standard procedures at $30^{\circ} \mathrm{C}$ in YP medium (1\% yeast extract, $2 \%$ peptone) supplemented with $2 \%$ glucose (YPD), 3\% glycerol (YPG) or 2\% galactose (YPGal) shaking at 160-220 rpm (Curran \& Bugeja, 2006). $300 \mathrm{mg} / \mathrm{L}$ G418 sulphate was added for selection of $K A N^{+}$cells. Selective minimal media for transformation with auxotrophic marker genes and maintenance of plasmid-carrying strains contained $0.67 \%$ yeast nitrogen base without amino acids (YNB), 0.07\% complete supplement mixture (CSM) lacking the appropriate metabolite and $2 \%$ glucose (SD), 3\% glycerol (SG), 3\% lactate (pH 5.0 using KOH, SLac) or $2 \%$ galactose (SGal). For multiple selection CSM lacking uracil, tryptophan, histidine and leucine was used and supplemented with metabolites ( $0.03 \mathrm{~g} / \mathrm{L} \mathrm{L}$-leucine, $0.02 \mathrm{~g} / \mathrm{L}$ uracil/ tryptophan/ histidine) to the desired mixture. Temperature sensitive mutants were grown at $25^{\circ} \mathrm{C}$ and shifted to restrictive temperature $\left(37^{\circ} \mathrm{C}\right)$ for the last $3 \mathrm{~h}$ of growth. Plates were supplemented with $28 \mathrm{~g} / \mathrm{L}$ agar.

Yeast strains were preserved as cryo stocks by mixing $800 \mu \mathrm{l}$ of an over night culture in YPD or SD with $200 \mu \mathrm{l}$ sterile $80 \%$ glycerol and stored at $-80^{\circ} \mathrm{C}$.

\subsubsection{Growth conditions for $E$. coli}

E. coli XL1 Blue were grown according to standard procedures in lysogeny broth (LB: $1 \% \mathrm{NaCl}, 0.5 \%$ yeast extract, $1 \%$ tryptone) at $37^{\circ} \mathrm{C}$ (Sambrook \& Russell, 2001 ). Plates were supplemented with $15 \mathrm{~g} / \mathrm{L}$ agar. $0.1 \mathrm{~g} / \mathrm{L}$ ampicillin was added for selection. Plasmid bearing E. coli were preserved as cryo stocks by mixing $800 \mu \mathrm{l}$ of an over night culture with $200 \mu \mathrm{l}$ sterile $80 \%$ glycerol and stored at $-80^{\circ} \mathrm{C}$.

\subsubsection{Growth tests for yeast}

In order to compare growth of different strains on solid media, cells were raised over night in YP or an appropriate selective medium supplemented with $2 \%$ galactose of $0.5 \%$ glucose. The cultures were diluted to an optical density (measured at $600 \mathrm{~nm} ; \mathrm{OD}_{600}$ ) of 0.5 , grown for $2 \mathrm{~h}$, washed in medium lacking a carbon source and finally spotted in a 10 fold serial dilution ( $\left.\mathrm{OD}_{600} 0.5-0.00005\right)$. Glucose was used 
as fermentable and lactate or glycerol as non-fermentable carbon sources. Plates were incubated at $30^{\circ} \mathrm{C}$ and $37^{\circ} \mathrm{C}$ for $2-5$ days.

In order to obtain more quantitative results, strains were compared in a growth test in liquid media. Here, cells were raised in YP containing $0.3 \%$ glucose over night, washed in YP without carbon source and diluted to $\mathrm{OD}_{600}=0.1$ in YPD and YPG respectively. From these dilutions quintruples of $200 \mu \mathrm{l} /$ well were added to a 96 well plate which was kept at $30^{\circ} \mathrm{C}$ and $700 \mathrm{rpm}$ on a Thermomixer for $24 \mathrm{~h}$ and monitored by measurements at $595 \mathrm{~nm}$ in a microplate reader every $2 \mathrm{~h}$. Mean growth rate and standard deviation were calculated for each quintruple.

\subsubsection{Generation of yeast strains lacking mitochondrial DNA ( rho $\left.^{0}\right)$}

To obtain yeasts lacking mitochondrial DNA ( $\mathrm{rho}^{0}$ strains), cells were grown in liquid YPD supplemented with $25 \mu \mathrm{g} / \mathrm{ml}$ ethidium bromide for 3 days in total, being diluted every day 1:60 in fresh medium (modified from Simon \& Faye, 1984). $15 \mu \mathrm{l}$ of the last culture were plated on YPD without ethidium bromide, grown for 2 days and tested for the absence of mitochondrial translation products and respiration by western blotting and growth tests on YPG.

\subsubsection{Whole cell lysate of yeast}

In order to analyze the protein content of whole cells, as adapteded from J. S. Cox (Cox, Chapman, \& Walter, 1997), yeast was grown in YPD or YPGal (if appropriate in SD or SGal) to late log phase. An equivalent of $\mathrm{OD}_{600}=1$ was harvested, resuspended in water and subjected to alkaline lysis by addition of $255 \mathrm{mM} \mathrm{NaOH}$ and $148 \mathrm{mM}$ $\beta$-mercaptoethanol and $10 \mathrm{~min}$ incubation on ice. Proteins were precipitated using TCA in a final concentration of $15 \%$ and $30 \mathrm{~min}$ to $12 \mathrm{~h}$ incubation at $-20^{\circ} \mathrm{C}$. Proteins were spun down at $12000 \mathrm{rpm}$ for $30 \mathrm{~min}$ at $4^{\circ} \mathrm{C}$, washed once in acetone and resuspended in $50 \mu \mathrm{l}$ X SDS sample buffer containing $10 \mathrm{mM}$ Tris base. Samples were subjected to SDS-PAGE analysis (see 5.4.1).

\subsubsection{Isolation of mitochondria}

For isolation of mitochondria, yeast was grown in YPG or SG (in case of strains with a defect in respiration on YPGal or SGal) for at least three days, increasing the total volume of fresh medium every day to a final volume of $2 \mathrm{~L}$ per flask and a final $\mathrm{OD}_{600}$ of 1.5-2.0. Isolation procedure was performed essentially as described previously 
(Meisinger, Pfanner, \& Truscott, 2006). Whole cultures were harvested for $15 \mathrm{~min}$ at $4700 \mathrm{rpm}$ (Sorvall H-12000/ Sorvall RC 12BP) and washed in water once (Avanti $\mathrm{J}-26 \mathrm{XP} / \mathrm{JA}-10)$. Pellets were incubated for $20 \mathrm{~min}$ at $30^{\circ} \mathrm{C}$ in $2 \mathrm{ml}$ buffer A (100 mM Tris/ pH 9.4 using $\mathrm{H}_{2} \mathrm{SO}_{4}, 1.54 \mathrm{~g} / \mathrm{L}$ dithiotreitol (DTT)) per g cell wet weight, then harvested at $4000 \mathrm{rpm}$ for $8 \mathrm{~min}$ and washed in $150 \mathrm{ml}$ buffer B $(20 \mathrm{mM} \mathrm{KPi} \mathrm{pH} \mathrm{7.4,}$ $1.2 \mathrm{M}$ sorbitol). Pellets were resuspended in $7 \mathrm{ml} / \mathrm{g}$ buffer B containing $4 \mathrm{mg} / \mathrm{g}$ zymolyase (Seikagaku Biobusiness Corporation/ Nacalai Tesque Inc.) and incubated for $60-90 \mathrm{~min}$ at $30^{\circ} \mathrm{C}$. Spheroblasts were spun down at $3000 \mathrm{rpm}$ for $8 \mathrm{~min}$ and washed in $150 \mathrm{ml}$ buffer B without enzyme. Pellets were then resuspended in $7 \mathrm{ml} / \mathrm{g}$ cold homogenization buffer (0.6 M sorbitol, $10 \mathrm{mM}$ Tris/ pH 7.4 using $\mathrm{HCl}, 1 \mathrm{mM}$ EDTA, $2 \mathrm{~g} / \mathrm{L}$ BSA, $1 \mathrm{mM}$ PMSF). The suspension was homogenized using a $60 \mathrm{ml}$ dounce homogenizer (potter) at $800 \mathrm{rpm}$ for 20 strokes on ice. The homogenate was centrifuged for $5 \mathrm{~min}$ at $3000 \mathrm{rpm}$ at $4^{\circ} \mathrm{C}$ and the resulting supernatant cleared again in a $10 \mathrm{~min}$ spin at $4000 \mathrm{rpm}$ at $4^{\circ} \mathrm{C}$. The crude mitochondrial fraction was pelleted by centrifugation for $15 \mathrm{~min}$ at $12,000 \mathrm{rpm}$ at $4^{\circ} \mathrm{C}$ (Avanti J-26 XP/ JA-20). Mitochondria were pooled in $5 \mathrm{ml}$ SEM buffer (250 mM sucrose, $20 \mathrm{mM}$ MOPS/ pH 7.2 using KOH, $1 \mathrm{mM}$ EDTA) containing $1 \mathrm{mM}$ PMSF and centrifuged again (12000 $\left.\mathrm{rpm}, 4^{\circ} \mathrm{C}, 15 \mathrm{~min}\right)$. Mitochondrial pellets were resuspended in a small amount of SEM for adjustment of protein concentration to $10 \mathrm{mg} / \mathrm{ml}$ using Bradford assay (see 5.4.3) and storage in small aliquots at $-80^{\circ} \mathrm{C}$.

For the fractionation experiment shown in Figure 2-2, additional samples were taken during the isolation procedure. Total represents yeast cells after homogenization. The postmitochondrial supernatant represent the supernatant of the first $12,000 \mathrm{rpm}$ spin.

\subsection{Molecular biology methods}

\subsubsection{Plasmid isolation}

Plasmids were purified from $2 \mathrm{ml}$ E. coli culture using Wizard ${ }^{\circledR}$ Plus SV Minipreps DNA Purification System (Promega), following the manufacturers specifications and resuspended in water. Concentration of nucleic acids was measured with NanoVue Plus Spectrophotometer (GE Healthcare). Plasmid DNA was stored at $-20^{\circ} \mathrm{C}$. 


\subsubsection{Yeast genomic DNA isolation}

For isolation of yeast genomic DNA, from a $10 \mathrm{ml}$ over night culture in YPD an amount corresponding to $\mathrm{OD}=2.5$ was harvested and resupended in $150 \mu \mathrm{l}$ Solution A $(50 \mathrm{mM}$ Tris/HCl pH 7.5, $10 \mathrm{mM}$ EDTA, 0.3\% $\beta$-mercaptoethanol, $0.5-0.25 \mathrm{mg} / \mathrm{ml}$ Zymolyase), and incubated at $37^{\circ} \mathrm{C}$ for $1 \mathrm{~h}$ (slightly shaking in Thermomixer). Sequentially, $10 \mu \mathrm{l}$ 10\% SDS and $100 \mu \mathrm{l} 8 \mathrm{M}$ ammonium acetate were added. After $15 \mathrm{~min}$ at $-20^{\circ} \mathrm{C}$ and a spin at $14000 \mathrm{rpm}$ and $4^{\circ} \mathrm{C}$ for $15 \mathrm{~min} 180 \mu \mathrm{l}$ of the supernatant was mixed with $120 \mu$ isopropanol for precipitation of DNA. DNA was pelleted at $14000 \mathrm{rpm}$ and $4^{\circ} \mathrm{C}$ for $15 \mathrm{~min}$, washed once in $70 \%$ ethanol, resuspended in $30 \mu \mathrm{l} \mathrm{TE}$ buffer (10 $\mathrm{mM}$ Tris/HCl pH 7.5, $1 \mathrm{mM}$ EDTA) and stored at $20^{\circ} \mathrm{C}$.

\subsubsection{PCR}

DNA segments were amplified from yeast genomic DNA or plasmids by polymerase chain reaction (PCR) with KOD polymerase (Merck Millipore). According to the manufacturers instructions, $1 \mathrm{X}$ KOD buffer, $1.5 \mathrm{mM} \mathrm{MgSO}_{4}, 0.2 \mathrm{mM}$ of each desoxynucleotide (dNTP), 0.3 $\mu \mathrm{M}$ forward and reverse primer, 10-100 ng template DNA and $1 \mathrm{U}$ of KOD polymerase were mixed per reaction. Cycling conditions were $2 \mathrm{~min}$ at $95^{\circ} \mathrm{C}$ for polymerase activation followed by 10 cycles of denaturation $(20 \mathrm{~s}$ at $\left.95^{\circ} \mathrm{C}\right)$, annealing $\left(10 \mathrm{~s}\right.$ at $52-56^{\circ} \mathrm{C}$ depending on the used primer pair) and extension $\left(15-25 \mathrm{~s} / \mathrm{kb}\right.$ at $70^{\circ} \mathrm{C}$ depending on the target size). In the following 20 cycles annealing temperature was lowered to $48-52^{\circ} \mathrm{C}$. Reaction was completed by a final extension for $1 \mathrm{~min}$ at $70^{\circ} \mathrm{C}$. PCR fragments were analyzed by agarose gel electrophoresis (1\% agarose in $1 \mathrm{X}$ TAE buffer $(40 \mathrm{mM}$ Tris, $20 \mathrm{mM}$ acetic acid and $1 \mathrm{mM}$ EDTA)) in horizontal electrophoresis cell (BioRad) at $100-120 \mathrm{~V}$ for 20-30 min. DNA was isolated from the gel or purified directly from the PCR tube using Wizard ${ }^{\circledR}$ SV Gel and PCR Clean-Up System (Promega) as described by the manufacturers. Concentration was determined using NanoVue Plus Spectrophotometer (GE Healthcare) and fragments stored in water at $-20^{\circ} \mathrm{C}$.

\subsubsection{Cloning}

Cloning of plasmids listed in TABLE was carried out according to standard procedures (Sambrook \& Russell, 2001). To this end, for insertion of purified PCR 
products in plasmids, both were first digested using appropriate FastDigest restriction enzymes (Thermo Fisher Scientific). In a $30 \mu$ reaction $1 \mathrm{X}$ reaction buffer, $1 \mu \mathrm{l}$ of each enzyme and $\sim 1 \mu \mathrm{g}$ of DNA were mixed and incubated for $30 \mathrm{~min}$ at $37^{\circ} \mathrm{C}$. After 5-10 min heat inactivation (depending on the enzyme), digested plasmids were analyzed by agarose gel electrophoresis. Linearized plasmid as well as insert were purified using Wizard ${ }^{\circledR}$ SV Gel and PCR Clean-Up System and used for ligation with Rapid Ligation Kit (Thermo Fisher Scientific). To this end, $100 \mathrm{ng}$ plasmid, an adequate amount of insert, $4 \mu \mathrm{l}$ DNA ligation buffer and $1 \mu \mathrm{l}$ T4 DNA ligase were mixed. The amount of insert was calculated as follows: mass $\operatorname{Ins}[\mathrm{ng}]=5 \mathrm{x}$ mass $_{V e c}[\mathrm{ng}] \mathrm{x}$ length $_{\text {Ins }}[\mathrm{bp}] /$ length $_{\text {Vec }}[\mathrm{bp}]$. After an incubation of $30 \mathrm{~min}$ at $21^{\circ} \mathrm{C}$, $10 \mu \mathrm{l}$ of the reaction was used for transformation (see 5.3.7). Constructs were analyzed by analytical restriction digest and further confirmed by sequencing (Seqlab Sequence Laboratories, Göttingen, Germany).

\subsubsection{In vitro mutagenesis}

In order to introduce defined mutations into plasmids, such as mutations for amino acid exchanges, insertion of short sequences coding for a small tag or removal of whole parts of the gene, the QuikChange Lightning Site-Directed Mutagenesis Kit (Agilent Technologies) was used. For this PCR-based approach primers were designed according to the manufacturers instructions with the desired mutation in the middle of the primer and $\sim 10-15$ bases of correct sequence on both sides. $125 \mathrm{ng}$ of each primer were mixed with $1 \mathrm{X}$ reaction buffer, 10-100 ng DNA template, $1 \mu \mathrm{l}$ dNTP mix and $1 \mu \mathrm{l}$ QuikChange Lightening Enzyme in a total volume of $50 \mu \mathrm{l}$. Cycling conditions were as follows: $2 \mathrm{~min}$ at $95^{\circ} \mathrm{C}$ for polymerase activation followed by 18 cycles of $20 \mathrm{~s}$ at $95^{\circ} \mathrm{C}$ for denaturation, $10 \mathrm{~s}$ at $60^{\circ} \mathrm{C}$ for annealing and $30 \mathrm{~s} / \mathrm{kb}$ of plasmid length at $68^{\circ} \mathrm{C}$ for elongation. Reaction was completed by a final elongation step for $5 \mathrm{~min}$ at $68^{\circ} \mathrm{C}$. For removal of parental DNA, incubated for $5 \mathrm{~min}$ at $37^{\circ} \mathrm{C}$ with $2 \mu \mathrm{l}$ Dnp I restriction enzyme, which was directly added to the amplification reaction. Proceeded with transformation into competent XL1 Blue.

\subsubsection{Transformation of $E$. coli}

Preparation of chemically competent E. coli was adapted from D. Hanahan and colleagues (Hanahan, 1983). $100 \mathrm{ml}$ of culture with an $\mathrm{OD}_{600}$ of 0.6 was harvested by centrifugation for $5 \mathrm{~min}$ at $3300 \mathrm{rpm}$ after an incubation on ice for $5 \mathrm{~min}$. Pellets 
were resuspended in $40 \mathrm{ml}$ ice cold buffer A $(30 \mathrm{mM} \mathrm{KAc}, 100 \mathrm{mM} \mathrm{RbCl}, 10 \mathrm{mM}$ $\mathrm{CaCl}_{2}, 50 \mathrm{mM} \mathrm{MnCl}_{2}, 15 \%$ glycerol, pH 5.8) and chilled on ice for another $5 \mathrm{~min}$. Cells were harvested again, resuspended in $4 \mathrm{ml}$ buffer B (10 mM MOPS, $75 \mathrm{mM} \mathrm{CaCl}_{2}$, $10 \mathrm{mM} \mathrm{RbCl}_{2}, 15 \%$ glycerol, $\mathrm{pH} 6.5$ ), aliquoted to $100 \mu \mathrm{l}$ and stored at $-80^{\circ} \mathrm{C}$. For transformation cells were defrosted on ice, mixed with $10 \mu$ ligation mix, $10 \mu \mathrm{l}$ of Dnp I treated QuickChange reaction or $200 \mathrm{ng}$ plasmid DNA and incubated for $15 \mathrm{~min}$ on ice. After a brief heat shock for $1 \mathrm{~min}$ at $42^{\circ} \mathrm{C}$, cells were chilled on ice for another $5 \mathrm{~min}$, shaken for $1 \mathrm{~h}$ at $37^{\circ} \mathrm{C}$ with $1 \mathrm{ml} \mathrm{LB}$ medium and plated on ampicillin-containing selective plates.

\subsubsection{Transformation of yeast}

For transformation of yeast a strongly modified protocol based on a description by R. D. Gietz and colleagues (Gietz \& Woods, 2002) was used. To this end, an over night culture of yeast in YPD was diluted to $\mathrm{OD}_{600}=0.4$ in $40 \mathrm{ml}$ YPD in the morning and further grown for at least $4 \mathrm{~h}$. Cells were harvested at $3500 \mathrm{rpm}$ for $3 \mathrm{~min}$ and washed in water once. Afterwards, pellets were resuspended in $5 \mathrm{ml} 0.1 \mathrm{M} \mathrm{LiAc}$, aliquoted into $100 \mu \mathrm{l}$ and kept at room temperature (RT) for $10 \mathrm{~min}$. Meanwhile, $10 \mathrm{~g} / \mathrm{l}$ herring sperm DNA was boiled at $95^{\circ} \mathrm{C}$ for $5 \mathrm{~min}$ and immediately chilled on ice for $5 \mathrm{~min}$. $100 \mu \mathrm{l}$ competent cells, $10 \mu \mathrm{l}$ herring sperm DNA, $360 \mu \mathrm{l}$ PEG 4000 (40\% (w/v)) and 1-5 $\mu \mathrm{l}$ of plasmid DNA or PCR fragment were mixed and incubated $30 \mathrm{~min}$ at $30^{\circ} \mathrm{C}$ under mild agitation ( $450 \mathrm{rpm}$ ). After addition of $72 \mu \mathrm{l}$ DMSO, cells were shifted to $42^{\circ} \mathrm{C}$ for $30 \mathrm{~min}$, then harvested $3 \mathrm{~min}$ at $4000 \mathrm{rpm}$ and resuspended in $500 \mu \mathrm{l}$ YPD. Incubation was resumed at $30^{\circ} \mathrm{C}$ at $750 \mathrm{rpm}$ for $30 \mathrm{~min}$ in case of transformation with plasmids and auxotrophy marker genes and for at least $60 \mathrm{~min}$ in case of antibiotic resistance genes. Cells were then harvested again $3 \mathrm{~min}$ at $4000 \mathrm{rpm}$, resuspended in a small amount of water and spread on selective plates. Transformants had to undergo a second round of single colony selection before they were propagated for cryo stocks and further experiments.

\subsubsection{Chromosomal deletions and insertions in yeast}

Chromosomal deletions and insertion of C-terminal tags in yeast was achieved by a PCR based strategy (Janke et al., 2004; Knop et al., 1999; Longtine et al., 1998). For deletions, HIS3MX6, kanMX6, TRP1 (Longtine et al., 1998) or natNT1 (Janke et al., 2004) cassettes were amplified with primers containing homology regions to up- 
and downstream sequences of the indicated open reading frame. For insertion of ZZ and SF tag, pYM10 (Knop et al., 1999) and pYM2.2 (Janke et al., 2004) served as a template. Homology regions of the primers in this case matched the end of the indicated open reading frame and a downstream sequence. PCR products were used for transformation as described in 5.3.7. Integration into the genome was confirmed by PCR and wester blotting.

\subsubsection{In vitro transcription and translation}

In order to generate $\left[{ }^{35} \mathrm{~S}\right]$-labeled precursers and protein fragments, first capped mRNAs were produced using the mMASSAGE mMACHINE SP6 kit (Promega). PCR products containing a SP6 promoter in front of the ORF were used as template for the in vitro transcription reaction. In brief, $1 \mathrm{x}$ NTP/CAP, $1 \mathrm{x}$ reaction buffer, $1 \mu \mathrm{g}$ PCR product and $2 \mu \mathrm{l}$ enzyme mix were mixed for a $20 \mu \mathrm{l}$ reaction and incubated for $90 \mathrm{~min}$ at $37^{\circ} \mathrm{C}$. For removal of DNA template, samples were subsequently incubate with $2 \mathrm{U}$ TURBO DNaseI for $15 \mathrm{~min}$ at $37^{\circ} \mathrm{C}$. RNA was recovered by precipitation through addition of $30 \mu \mathrm{l}$ nuclease-free water and $30 \mu \mathrm{LiCl}$ solution $(7.5 \mathrm{M}$ lithium choride, $50 \mathrm{mM}$ EDTA) and incubation at $-20^{\circ} \mathrm{C}$ for at least $30 \mathrm{~min}$. RNA was pelleted for $15 \mathrm{~min}$ at $14000 \mathrm{rpm}$ and $4^{\circ} \mathrm{C}$, washed with $1 \mathrm{ml} 70 \%$ ethanol, dried, resuspended in $50 \mu \mathrm{l}$ RNase-free water and stored at $-80^{\circ} \mathrm{C}$. RNA was then used for translation with the Flexi ${ }^{\circledR}$ Rabbit Reticulocyte Lysate System (Promega). To this end, $33 \mu \mathrm{l} \mathrm{Flexi}{ }^{\circledR}$ Rabbit Reticulocyte Lysate, $1 \mu \mathrm{l} 1 \mathrm{mM}$ amino acid mix without methionine, $1.5 \mu \mathrm{g}$ mRNA, 70-120 mM KCl, 0-2 mM MgAc, 0-2 mM DTT (depending on the protein synthesized, see Table 5-8) and $50 \mu \mathrm{Ci}\left[{ }^{35} \mathrm{~S}\right]-$ Met were mixed and incubated $90 \mathrm{~min}$ at $30^{\circ} \mathrm{C}$.

Alternatively, transcription and translation were performed in a coupled reaction from plasmid templates (see Table 5-4) using TNT ${ }^{\circledR}$ Quick Coupled Transcription Translation kit (Promega). This reaction was carried out in $40 \mu \mathrm{l}$ TNT ${ }^{\circledR}$ Quick Master Mix with $1 \mu \mathrm{g}$ plasmid DNA and $50 \mu \mathrm{Ci}\left[{ }^{35} \mathrm{~S}\right]-$ Met. 
MATERIAL AND METHODS

Table 5-8: Conditions for Flexi ${ }^{\circledR}$ Rabbit Reticulocyte Lysate System

\begin{tabular}{llll} 
Precursor & KCl & MgAc & DTT \\
\hline \hline Rcf1 & $70 \mathrm{mM}$ & $0.8 \mathrm{mM}$ & $2 \mathrm{mM}$ \\
Rcf2 (all variants) & $100 \mathrm{mM}$ & $0.8 \mathrm{mM}$ & $2 \mathrm{mM}$ \\
Rcf3 & $100 \mathrm{mM}$ & $1 \mathrm{mM}$ & $0 \mathrm{mM}$ \\
Cox13 & $70 \mathrm{mM}$ & $0.8 \mathrm{mM}$ & $0 \mathrm{mM}$ \\
Su9-DHFR & $100 \mathrm{mM}$ & $1 \mathrm{mM}$ & $0 \mathrm{mM}$
\end{tabular}

\subsection{Protein biochemistry methods}

\subsubsection{SDS-PAGE}

Separation of denatured proteins by SDS polyacrylamide gel electrophoresis (SDS-PAGE) was carried out based on the principles described by U. K. Laemmli. (Laemmli, 1970) but with several changes specified below. Depending on the size of the analyzed proteins, separating gel were prepared with different acrylamide concentrations (16-10\%) using a stock solution of $30 \% / 0.8 \%$ acrylamide/bisacrylamide (Gel Mix 30, Roth), 386 mM Tris/HCl pH 8.8, 0.1\% SDS, 0.0588\% APS and $0.0588 \%$ TEMED. The stacking gel contained 5\% acrylamide, $80 \mathrm{mM}$ Tris/ $\mathrm{HCl}$ pH 6.8, 0.1\% SDS, 0.1\% APS and 0.2\% TEMED. The running buffer contained $25 \mathrm{mM}$ Tris, $191 \mathrm{mM}$ glycine and $0.1 \%$ SDS. In order to obtain a better resolution for proteins smaller than $6 \mathrm{kDa}$, gels were supplemented with urea as suggested for Tricine gels by H.Schägger (Schägger, 2006). The separating gel contained $17.5 \%$ acrylamide (using a stock of $60 \% / 0.8 \%$ acrylamide/bis-acrylamide solution), $683 \mathrm{mM}$ Tris/HCl pH 8.8, $7.77 \mathrm{mM} \mathrm{NaCl}, 5.4 \mathrm{M}$ urea, 0.1\% SDS, $0.032 \%$ APS and $0.066 \%$ TEMED. The stacking gel contained 5.4\% acrylamide (using the same stock solution), $108 \mathrm{mM}$ Tris/HCl pH 6.8, $3.3 \mathrm{M}$ urea, 0.12\% SDS, 0.126\% APS and $0.11 \%$ TEMED. The running buffer consisted of $50 \mathrm{mM}$ Tris, $192 \mathrm{mM}$ glycine and 0.1\% SDS. All gels were run in custom-made midi chambers at $30 \mathrm{~mA}$ per gel for 3-5 h or at $5 \mathrm{~mA}$ per gel for $14-16 \mathrm{~h}$.

SDS sample buffer contained 2\% SDS, 10\% glycerol, $60 \mathrm{mM}$ Tris/HCl pH 6.8, 1\% $\beta$-mercaptoethanol and $0.01 \%$ bromphenolblue. As a molecular weight standards 
the unstained SDS-PAGE protein marker 6.4-200 kDa (SERVA) or the All Blue Prestained Protein Standards 10-250 kDa (BioRad) were used.

\subsubsection{Blue native PAGE}

Separation of native protein complexes by blue native polyacrylamide gel electrophoresis (BN-PAGE) was performed as initially described by Schägger and von Jagow and adapted by Dekker and colleagues (Dekker et al., 1997; Schägger \& Jagow, 1991). Separation gels contained 1x gel guffer (66.6 mM e-amino n-caproic acid and $50 \mathrm{mM}$ Bis-Tris/HCl pH 7.0), acrylamide from a 48\%/1.5\% acrylamide/bis-acrylamide stock solution, $0.08 \%$ APS and $0.133 \%$ TEMED. Usually, gradients of $4-10$ or $4-13 \%$ acrylamide were generated by mixing $4 \%$ and 10 / 13\% separation gel solutions (the latter containing $20 \%$ glycerol) with the help of a gradient mixer. Gels were cast and run in the SE600 Ruby system (Hoefer, GE Healthcare). The cathode buffer contained $50 \mathrm{mM}$ Tricine, $15 \mathrm{mM}$ Bis-Tris and 0.02\% Coomassie Brilliant Blue G250. Fors subsequent western blot and 2D analysis the cathode buffer was exchanged after $1 / 3$ of the run against Coomassie-free buffer. The anode buffer contained $50 \mathrm{mM}$ Bis-Tris/HCl pH 7.0. Unless not taken from IP or ZZ isolation procedures (see 5.5.1; 5.5.3; 5.5.4), samples were prepared by solubilisation of mitochondria in a buffer containing $20 \mathrm{mM}$ Tris/ $\mathrm{HCl} \mathrm{pH} \mathrm{7.4,} \mathrm{0.1-}$ $1 \mathrm{mM}$ EDTA, $50 \mathrm{mM} \mathrm{NaCl}, 10 \%$ glycerol, $1 \mathrm{mM}$ PMSF and either 1\% digitionin or $0.6 \% \mathrm{DDM}$ to $1 \mu \mathrm{g} / \mu \mathrm{l}$ for $20 \mathrm{~min}$ on ice. After removal of insoluble material by centrifugation at $14000 \mathrm{rpm}$ for $10 \mathrm{~min}$ at $4^{\circ} \mathrm{C}$ supernatant was mixed with $10 \mathrm{x}$ BN-loading dye (5\% Coomassie Brilliant Blue G250, 500 mM e-amino n-caproic acid and $100 \mathrm{mM}$ Bis-Tris/HCl pH 7.0). Samples were mixed well, kept on ice for $2 \mathrm{~min}$, spun down at $14000 \mathrm{rpm}$ for $1 \mathrm{~min}$ and loaded on the gel. Gel run was initiated at $15 \mathrm{~mA}$ per gel at $200 \mathrm{~V}$ and continued at $600 \mathrm{~V}$ after exchange of the cathode buffer. Gels were run at $4^{\circ} \mathrm{C}$ until the Coomassie front reached the bottom of the gel.

In case of 2D analysis, whole BN lanes were separated, incubated in SDS running buffer containing $50 \mathrm{mM}$ DTT for $30 \mathrm{~min}$ at RT and subsequently cast into a regular SDS gel following the above-described procedures for SDS-PAGE.

\subsubsection{Determination of protein concentration}

Protein concentration of mitochondrial samples was estimated following Bradford assay (Bradford, 1976) and using Roti ${ }^{\circledR}$-Quant (Roth) according to the 
manufacturers specifications. Bovine IgG was used as protein standard. Each sample was measured three times in different amounts from which a mean value was calculated.

\subsubsection{Western blotting and immunodetection}

Western blotting and immunodetection were carried out according to standard protocols (Gallagher, Winston, Fuller, \& Hurrell, 2004). PVDF membranes (Immobilon-P, Merck Millipore) were rinsed in methanol for activation. Blotting papers (BF2 grade, $190 \mathrm{~g} / \mathrm{m}^{2}$, Sartorius stedim), activated membrane and gel were soaked in transfer buffer (20 mM Tris, $150 \mathrm{mM}$ glycine, $0.02 \%$ SDS, 20\% ethanol) and assembled in a semi dry blotting chamber (Peqlab). Proteins were transferred to the membrane at $25 \mathrm{~V}$ and $250 \mathrm{~mA}$ for $2.5 \mathrm{~h}$ in case of midi-sized gels or for $3 \mathrm{~h}$ in case of BN gels. To visualize protein standard, membranes were stained in Coomassie (see 5.4.6). After complete destaining of membranes in methanol, they were blocked in a solution of $5 \%$ (or in case of BN 10\%) milk powder in TBS-T (20 mM Tris/ $\mathrm{HCl} \mathrm{pH} \mathrm{7.5,} 62 \mathrm{mM} \mathrm{NaCl}, 0.1 \%$ Tween 20) for $1 \mathrm{~h}$ at room temperature or at $4^{\circ} \mathrm{C}$ over night. Sera with specific primary antibodies were diluted in $5 \%$ milk in TBS-T and applied to the membranes for 1-2 $\mathrm{h}$ at room temperature or up to $14 \mathrm{~h}$ at $4^{\circ} \mathrm{C}$. Afterwards, membranes were rinsed in TBS-T three times for $10 \mathrm{~min}$ and incubated with secondary antibodies diluted in 5\% milk in TBS-T for $1 \mathrm{~h}$ at room temperature of up to $14 \mathrm{~h}$ at $4^{\circ} \mathrm{C}$. For dilutions of secondary antibodies see 5.1.2.

\subsubsection{Autoradiography}

In vitro synthesized $\left[{ }^{35} \mathrm{~S}\right]$-labeled proteins were transferred to PVDF membranes as described in 5.4.4. However, after Coomassie staining (5.4.6) membranes were dried completely. Alternatively, gels were not used for transfer but directly stained with Coomassie and dried between a plastic bag and two blotting papers on a vacuum gel drier (Scie-Plas) at $65^{\circ} \mathrm{C}$ for $2.5 \mathrm{~h}$. Both, dried membranes and gels were subsequently treated in the same way. Protein standard was marked with radioactive ink (standard fountain pen ink containing $100 \mu \mathrm{Ci}$ [35S]-Met per cartridge) and covered with adhesive tape. Gels or membranes were then exposed to Storage Phosphor Screens (GE Healthcare). Signals were digitized using the STORM820 scanner (GE Healthcare) and, if necessary, quantified with ImageQuantTL software (GE Healthcare) using rolling ball background subtraction. 


\subsubsection{Coomassie staining of membranes and gels}

For visualization of proteins after PAGE or western blotting, gels and membranes were stained in Coomassie stainer (40\% ethanol, 10\% acetic acid, $0.15 \%$ Coomassie Brilliant Blue R250) and destained (30\% ethanol, 10\% acetic acid) until protein bands were clearly distinguishable from the background.

\subsubsection{Steady state analysis of mitochondrial proteins}

To analyze and compare amounts of mitochondrial proteins at steady state, isolated mitochondria were subjected to SDS-PAGE analysis. To this end, isolated mitochondria were mixed with SDS sample buffer (see 5.4.1) to a final protein concentration of $1 \mathrm{mg} / \mathrm{ml}$ and incubated at $30^{\circ} \mathrm{C}$ and $1000 \mathrm{rpm}$ for $30 \mathrm{~min}$. Unless indicated otherwise 5 and $10 \mu \mathrm{g} /$ lane were loaded on SDS or Urea gels.

\subsubsection{Testing solubilization properties of mitochondria proteins}

To test the solubilization properties and stability of FLAGRcf2 ${ }^{\mathrm{N}}$, mitochondrial pellets were resuspended in regular BN solubilization buffer $(20 \mathrm{mM}$ Tris/ $\mathrm{HCl}$ pH 7.4, 0.1$1 \mathrm{mM}$ EDTA, $50 \mathrm{mM} \mathrm{NaCl}, 10 \%$ glycerol, $1 \mathrm{mM}$ PMSF) containing either $1 \%$ digitonin, $0.6 \%$ DDM, $0.5 \%$ Triton X-100 or $1 \%$ SDS. Samples were incubated on ice for $20 \mathrm{~min}$ and cleared at $4^{\circ} \mathrm{C}$ and $20,000 \mathrm{xg}$ for $10 \mathrm{~min}$. The supernatants were transferred to a new tube, while the remaining pellets were again resuspended in solubilization buffer. Both samples were mixed with SDS sample buffer. As positive control for detection of FLAGRcf2 ${ }^{N}$, one sample was resuspended in regular SDS sample buffer (see 5.4.1) and kept at room temperature for the course of the experiment. $10 \mu \mathrm{g}$ of sample / lane was analyzed via SDS-PAGE.

\subsubsection{Defining sub-mitochondrial protein localization}

In order to distinguish between integral membrane, membrane-associated and soluble proteins, carbonate extractions were performed essentially as described earlier (Vukotic et al., 2012). In brief, isolated mitochondria were incubated in $0.1 \mathrm{M}$ $\mathrm{Na}_{2} \mathrm{CO}_{3}(\mathrm{pH} 11.5$ or $\mathrm{pH} 10.8)$ or $1 \%$ Triton X-100 supplemented with $150 \mathrm{mM} \mathrm{NaCl}$ for $20 \mathrm{~min}$ and centrifuged at $45000 \mathrm{rpm}$ at $4^{\circ} \mathrm{C}$ for $45 \mathrm{~min}$. Samples were then precipitated with TCA (see 5.2.5) and subjected to SDS-PAGE analysis. For information on the mitochondrial compartment the protein is located in, protease protection assays were carried out as described by M. Vukotic and colleagues as well 
(Vukotic et al., 2012). Here, isolated mitochondria were converted to mitoplasts by hypotonic swelling in EM buffer (1 mM EDTA, $10 \mathrm{mM}$ MPOS [pH 7.2 with $\mathrm{KOH}$ ]), kept intact in SEM buffer (additional $250 \mathrm{mM}$ sucrose) or lysed in 1\% Triton X-100 and subsequently treated with Proteinase K. Samples were precipitated with TCA and subjected to SDS-PAGE.

\subsubsection{Gel filtration}

As an alternative, providing a higher resolution than the above-described gradients, gel filtration analysis was performed with digitonin-solubilized mitochondria. After solubilization samples of $200 \mu \mathrm{g}$ were loaded on a Superose 6 column equilibrated with the same solubilization buffer containing $0.1 \%$ digitonin. Fractions of $1 \mathrm{ml}$ were collected, precipitated with TCA (see 5.2.5), resuspended in SDS sample buffer and subjected to SDS-PAGE analysis (see 5.4.1).

\subsection{Purification of proteins and protein complexes}

\subsubsection{IgG chromatography}

For native isolation of ZZ-tagged proteins IgG chromatography was performed based on protocol published by P. Rehling and colleagues (Rehling et al., 2003) with modifications as specified by B. Bareth and coworkers (Bareth et al., 2013). In brief, mitochondria were solubilized in a buffer $(20 \mathrm{mM}$ Tris pH 7.4, $0.5 \mathrm{mM}$ EDTA, $100 \mathrm{mM} \mathrm{NaCl}, 5 \%$ Glycerol, $2 \mathrm{mM}$ PMSF) containing either 1\% digitonin or $0.6 \%$ DDM for $40 \mathrm{~min}$ and unsolublized material was removed by a $10 \mathrm{~min}$ spin at $14000 \mathrm{rpm}$ at $4^{\circ} \mathrm{C}$. After saving a small sample as a total, the protein complexes of the supernatant were bound to IgG sepharose (human IgGs coupled to $\mathrm{CNBr}$ activated sepharose according to the manufacturers protocol [GE Healthcare]) via the protein A part of the $\mathrm{ZZ}$ tag for $1.5 \mathrm{~h}$ at $4^{\circ} \mathrm{C}$. After several rounds of washing in wash buffer (for composition see solubilization buffer) containing $0.3 \%$ digitonin or 0.6\% DDM, complexes were eluted natively by AcTEV protease (Tobacco Etch Virus protease, $10 \mathrm{U} / \mu \mathrm{l}$ ) cleavage over night at $8^{\circ} \mathrm{C}$. The protease cleavage site is included between bait and tag, as designed in KNOP et al 1999. The protease itself, carrying a polyhistidine, was remove by addition of Ni-NTA (Quiagen) pre-equilibrated with wash buffer. TEV-cleaved native eluates were mixed either with SDS sample buffer 
or with BN sample buffer and analysed by SDS-PAGE or BN-PAGE / 2D and western blotting. In case of the two-step purification after copper cross-linking, the isolation was performed from DDM-solubilized mitochondria and TEV eluates were used for subsequent Rcf2 immunoprecipitation.

\subsubsection{Crosslinking of antibodies to PA-Sepharose}

For co-immunoprecipitiation, specific antisera were bound to protein A-sepharose (GE Healthcare) as described earlier (Bareth et al., 2013). Antisera were diluted 1:8 (in case of Rcf1 sera 1:4) in $0.1 \mathrm{M}$ potassium phosphate buffer (pH 7.4) and incubated with protein A-sepharose for $1 \mathrm{~h}$ at room temperature. Beads were washed in $0.1 \mathrm{M}$ sodium borate $(\mathrm{pH} \mathrm{9.0)}$ and subsequently cross-linked with $5 \mathrm{mg} / \mathrm{ml}$ dimethyl pimelimidate (DMP) solution in $0.1 \mathrm{M}$ sodium borate $(\mathrm{pH} 9.0)$ for $30 \mathrm{~min}$ at room temperature. Reaction was quenched with $1 \mathrm{M}$ Tris/ $\mathrm{HCl} \mathrm{pH} 7.4$ at $4^{\circ} \mathrm{C}$ over night. Finally, beads were washed in TBS-T for at least 3 times and stored at $4^{\circ} \mathrm{C}$ in TBS-T containing $2 \mathrm{mM}$ sodium acid. Prior to and after each immunoprecipitation beads were washed at room temperature two times with acetate buffer (pH 3.4) followed by three times with TBS-T. All centrifugation steps with beads were performed at $100 \mathrm{x} g$.

\subsubsection{Immunoprecipitation}

Immunoprecipitation (IP) was performed for Rcf1, Rcf2, Rcf3, Qcr8 and Cox2 as described (Bareth et al., 2013; Hutu et al., 2008; Mick et al., 2010) but with minor changes. As specificity control either a respective deletion strain or an antibody against the unrelated protein Pam18 were used. In detail, mitochondria isolated from the indicated strains were lysed in $20 \mathrm{mM}$ Tris (pH 7.4), $80 \mathrm{mM} \mathrm{NaCl}, 0.5 \mathrm{mM}$ EDTA, 1\% digitonin, 10\% glycerol and $1 \mathrm{mM}$ PMSF for $40 \mathrm{~min}$ under agitation. Lysates were cleared at 20,000x $\mathrm{g}$ for $10 \mathrm{~min}$ at $4^{\circ} \mathrm{C}$ and input samples (total) were taken. Lysates were split to indicated antibody-coupled sepharose columns and incubated for $1.5 \mathrm{~h}$ at $4^{\circ} \mathrm{C}$. After eight rounds of washing in the buffer indicated above, containing $0.3 \%$ digitonin, precipitated protein complexes were eluted in an appropriate volume of $0.1 \mathrm{M}$ glycine ( $\mathrm{pH} 2.8$ ). Eluates and total samples were analysed by SDS-PAGE and western blotting.

In case of the two-step purification after copper cross-linking a denaturing IP of Rcf2 was performed. $0.6 \%$ DDM-containing TEV eluates from Cox4zZ isolation were 
mixed with a second lysis buffer (20 mM Tris pH 7.4, $80 \mathrm{mM} \mathrm{NaCl,} \mathrm{0.5} \mathrm{mM} \mathrm{EDTA,}$ $10 \%$ glycerol, $2 \mathrm{mM}$ PMSF, 1\% Triton X-100) to a final concentration of $0.6 \%$ Triton X-100. After $20 \mathrm{~min}$ on ice lysates were cleared at $20,000 \mathrm{xg}$ and subjected to the IP procedure described above. The wash buffer contained $0.3 \%$ Triton X-100.

\subsubsection{FLAG isolation}

For isolation of FLAG tagged Rcf2, $20 \mu \mathrm{l}$ ANTI-FLAG M2 affinity gel (Sigma Aldrich) per $500 \mu \mathrm{g}$ mitochondria was used. Conditions for solubilization, binding and washing were chosen as described for immunoprecipiation (see 5.5.3). Bound protein complexes were eluted natively by incubation with FLAG ${ }^{\circledR}$ peptide (Sigma Aldrich) according to the manufacturers specifications. Total, unbound and elution were analyzed on regular SDS-PAGE as well as on BN-PAGE followed by second dimension.

\subsection{Specialized assays}

\subsubsection{In vitro protein import and assembly assay}

Radiolabeled precursor proteins $(\max 10 \%[\mathrm{v} / \mathrm{v}])$ were imported into isolated mitochondria essentially as described by M. T. Ryan and coworkers (Ryan, Voos, \& Pfanner, 2001). Mitochondria were incubated in import buffer (250 mM sucrose,

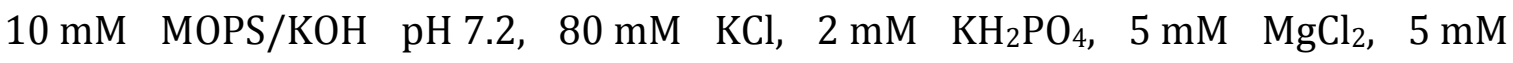
methionine, $1 \%$ fatty-acid free BSA) supplemented with $2 \mathrm{mM}$ ATP and NADH at $25^{\circ} \mathrm{C}$. For imports with subsequent assembly $0.1 \mathrm{mg} / \mathrm{ml}$ creatine kinase and $5 \mathrm{mM}$ creatin phosphate were added as ATP regenerating system. As a control for membrane potential-depended import and to stop reactions after indicated times, the membrane potential was dissipated using $1 \mu \mathrm{M}$ valinomycin, $8 \mu \mathrm{M}$ antimycin $\mathrm{A}$ and $20 \mu \mathrm{M}$ oligomycin (AVO). After placing samples on ice unimported precursor proteins were removed with $50 \mathrm{mg} / \mathrm{L}$ proteinase $\mathrm{K}$ for $10 \mathrm{~min}$. Protease was inactivated with $2 \mathrm{mM}$ PMSF for $10 \mathrm{~min}$ on ice. Samples were centrifuged for $10 \mathrm{~min}$ at $14000 \mathrm{rpm}$ at $4^{\circ} \mathrm{C}$ and washed in SEM (see 5.2.6) containing $2 \mathrm{mM}$ PMSF. For subsequent SDS-PAGE analysis, samples were resuspened in SDS sample buffer (see 5.4.1). For BN-PAGE analysis, samples were solubilized in $1 \%$ digitonin or $0.6 \%$ 
DDM as described in SEE. Both were further processed for autoradiography (see 5.4.5). Alternatively, SEM-washed samples were used for further experiments (see 5.6.2).

\subsubsection{Modification of cysteines using PEG maleimid}

The ability of PEG maleimid (average $M_{n}$ 2000, Sigma Aldrich) to covalently bind cysteines, thereby increasing the mass of the modified protein by $2 \mathrm{kDa}$, was used to asses the oxidation state of cysteines as well as to confirm Rcf2 topology (Roberts, Bentley, \& Harris, 2002). In general, for this treatment mitochondria, re-isolated after Rcf2 import, were resuspended in a buffer containing 5 mM PEG maleimid and incubated for $1 \mathrm{~h}$. The reaction was quenched by incubation with $10 \mathrm{mM}$ DTT for 10 min on ice. Afterwards, samples were mixed with SDS sample buffer and subjected to SDS-PAGE analysis. The PEG maleimid-containing buffer was chosen adequately to the purpose of the experiment. If modification of fully denatured proteins was sought, it contained $50 \mathrm{mM}$ Tris pH 7.4, 1\% SDS, $1 \mathrm{mM}$ EDTA, 1X cOmplete (EDTA free, Roche), $0.1 \mathrm{mg} / \mathrm{ml}$ BSA and $0.2 \mathrm{mM}$ PMSF. Incubation in this buffer took place at room temperature. In case only IMS exposed cysteine residues were targeted, the buffer contained 1 mM EDTA, 10 mM MOPS (pH 7.2 with $\mathrm{KOH}$ ) and incubation took place on ice.

\subsubsection{Protease inhibitor treatment of mitochondria}

In order to determine the protease class responsible for the Rcf2 processing step, $\operatorname{Rcf} \mathrm{C}$ was monitored after import into mitochondria pretreated with different inhibitors. To this end mitochondria resuspended in import buffer supplemented with $2 \mathrm{mM}$ ATP and NADH as described in 5.6.1 were treated with individual or mixed inhibitors in the following concentrations: 2x cOmplete, $10 \mathrm{mM}$ EDTA, $10 \mu \mathrm{M}$ pepstatin A. After 15 min on ice, samples were further handled as described in 5.6.1.

\subsubsection{Copper cross-linking}

Copper mediated cross-linking of cysteine containing neighboring proteins was performed essentially as described earlier (Falke \& Koshland, 1987; Kobashi, 1968; F. Y. Zeng, Hopp, Soldner, \& Wess, 1999), but with several modifications. To this end, isolated mitochondria were incubated with $2 \mathrm{mM} \mathrm{CuSO}_{4}$ in $\mathrm{SH}_{\text {buffer }}(0.6 \mathrm{M}$ sorbitol, $20 \mathrm{mM}$ HEPES pH 7.5) for $30 \mathrm{~min}$ on ice. Reaction was quenched by addition of 
$17 \mathrm{mM}$ NEM and $17 \mathrm{mM}$ EDTA and incubation on ice for $15 \mathrm{~min}$. Mitochondria were re-isolated at $14000 \mathrm{rpm}$ and $4^{\circ} \mathrm{C}$ for $10 \mathrm{~min}$ and resuspended in SDS sample buffer without $\beta$-mercaptoethanol for SDS-PAGE analysis or further processed for IgG chromatography and Rcf2 IP.

\subsubsection{Determination of enzyme activities in vitro}

Activity of cytochrome $c$ oxidase was determined by assaying the oxidation of cytochrome $c$ in isolated mitochondria as described earlier (Vukotic et al., 2012). The reaction was performed with an appropriate amount of mitochondria in $40 \mathrm{mM}$ potassium phosphate buffer ( $\mathrm{pH} 7.4$ ) and initiated by addition of $0.1 \%$ sodium dithionite-reduced cytochrome $c$ (Sigma). Decrease in absorbance at $550 \mathrm{~nm}$ was followed in a spectrophotometer. Activity of cytochrome $b c_{1}$ complex was determined similarly by assaying the reduction of cytochrome $c$. Here, the buffer additionally contained $0.5 \mathrm{mM} \mathrm{NADH}$ and $12.5 \mathrm{mM} \mathrm{KCN}$ and reaction was started by addition of $0.1 \%$ oxidized cytochrome c. Increase in absorbance at $550 \mathrm{~nm}$ was followed in a spectrophotometer. Concentration of cytochrome c was calculated using its extinction coefficient at $550 \mathrm{~nm}\left(21.84 \mathrm{mM}^{-1} \mathrm{~cm}^{-1}\right)$.

\subsubsection{Determination of oxygen consumption rates}

Oxygen consumption rates were measured in isolated mitochondria in an Oxygraph $2 \mathrm{k}$ (Oroboros) at $30^{\circ} \mathrm{C}$. Per measurement $2.1 \mathrm{ml}$ buffer (250 mM sucrose,

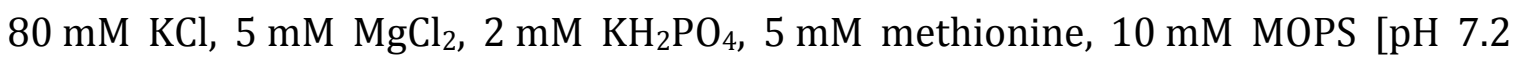
with $\mathrm{KOH}]$ ) were supplemented with $1 \mathrm{mM}$ ATP and $1 \mathrm{mM} \mathrm{NADH}$ and equilibrated for $5 \mathrm{~min}$ to obtain a stable oxygen concentration. Reactions were started by addition of $100 \mu \mathrm{g}$ of isolated mitochondria. 


\section{Bibliography}

Acin-Perez, R., \& Enriquez, J. A. (2014). The function of the respiratory supercomplexes: the plasticity model. Biochimica Et Biophysica Acta, 1837(4), 444-450. http://doi.org/10.1016/j.bbabio.2013.12.009

Acin-Perez, R., Bayona-Bafaluy, M. P., Fernandez-Silva, P., Moreno-Loshuertos, R., Pérez-Martos, A., Bruno, C., et al. (2004). Respiratory complex III is required to maintain complex I in mammalian mitochondria. Molecular Cell, 13(6), 805-815.

Acin-Perez, R., Fernandez-Silva, P., Peleato, M. L., Pérez-Martos, A., \& Enríquez, J. A. (2008). Respiratory active mitochondrial supercomplexes. Molecular Cell, 32(4), 529-539. http://doi.org/10.1016/j.molcel.2008.10.021

Akiyama, Y., \& Maegawa, S. (2007). Sequence features of substrates required for cleavage by GlpG, an Escherichia coli rhomboid protease. Molecular Microbiology, 64(4), 1028-1037. http://doi.org/10.1111/j.13652958.2007.05715.x

Allison, D. S., \& Schatz, G. (1986). Artificial mitochondrial presequences. Proceedings of the National Academy of Sciences, 83(23), 9011-9015.

Altmann, R. (1894). Die Elementarorganismen und ihre Beziehungen zu den Zellen.

Andersson, S. G., \& Kurland, C. G. (1998). Ancient and recent horizontal transfer events: the origins of mitochondria. APMIS. Supplementum, 84, 5-14.

Arnold, I., Pfeiffer, K., Neupert, W., Stuart, R. A., \& Schägger, H. (1998). Yeast mitochondrial F1F0-ATP synthase exists as a dimer: identification of three dimer-specific subunits. The EMBO Journal, 17(24), 7170-7178. http://doi.org/10.1093/emboj/17.24.7170

Arselin, G., Giraud, M.-F., Dautant, A., Vaillier, J., Brethes, D., Coulary-Salin, B., et al. (2003). The GxxxG motif of the transmembrane domain of subunit e is involved in the dimerization/oligomerization of the yeast ATP synthase complex in the mitochondrial membrane. European Journal of Biochemistry / FEBS, 270(8), 1875-1884. http://doi.org/10.1046/j.1432-1033.2003.03557.x

Balsa, E., Marco, R., Perales-Clemente, E., Szklarczyk, R., Calvo, E., Landázuri, M. O., \& Enríquez, J. A. (2012). NDUFA4 is a subunit of complex IV of the mammalian electron transport chain. Cell Metabolism, 16(3), 378-386. http://doi.org/10.1016/j.cmet.2012.07.015

Barbot, M., Jans, D. C., Schulz, C., Denkert, N., Kroppen, B., Hoppert, M., et al. (2015). Mic10 oligomerizes to bend mitochondrial inner membranes at cristae junctions. Cell Metabolism, 21(5), 756-763. http://doi.org/10.1016/j.cmet.2015.04.006 
Bareth, B., Dennerlein, S., Mick, D. U., Nikolov, M., Urlaub, H., \& Rehling, P. (2013). The heme a synthase Cox15 associates with cytochrome c oxidase assembly intermediates during Cox1 maturation. Molecular and Cellular Biology, 33(20), 4128-4137. http://doi.org/10.1128/MCB.00747-13

Bazán, S., Mileykovskaya, E., Mallampalli, V. K. P. S., Heacock, P., Sparagna, G. C., \& Dowhan, W. (2013). Cardiolipin-dependent reconstitution of respiratory supercomplexes from purified Saccharomyces cerevisiae complexes III and IV. The Journal of Biological Chemistry, 288(1), 401-411. http://doi.org/10.1074/jbc.M112.425876

Becker, T., Pfannschmidt, S., Guiard, B., Stojanovski, D., Milenkovic, D., Kutik, S., et al. (2008). Biogenesis of the mitochondrial TOM complex: Mim1 promotes insertion and assembly of signal-anchored receptors. Journal of Biological Chemistry, 283(1), 120-127. http://doi.org/10.1074/jbc.M706997200

Behrens, M., Michaelis, G., \& Pratje, E. (1991). Mitochondrial inner membrane protease 1 of Saccharomyces cerevisiae shows sequence similarity to the Escherichia coli leader peptidase. Molecular \& General Genetics : MGG, 228(1-2), 167-176.

Benda, C. (1898). Ueber die Spermatogenese der Vertebraten und hoeherer Evertebraten, II. Theil: Die Histiogenese der Spermien. Archiv Für Anatomie Und Physiologie, (73), 393-398.

Benz, R. (1994). Permeation of hydrophilic solutes through mitochondrial outer membranes: review on mitochondrial porins. Biochimica Et Biophysica Acta, 1197(2), 167-196.

Bohnert, M., Pfanner, N., \& van der Laan, M. (2015). Mitochondrial machineries for insertion of membrane proteins. Current Opinion in Structural Biology, 33, 92102. http://doi.org/10.1016/j.sbi.2015.07.013

Bohnert, M., Rehling, P., Guiard, B., Herrmann, J. M., Pfanner, N., \& van der Laan, M. (2010). Cooperation of stop-transfer and conservative sorting mechanisms in mitochondrial protein transport. Current Biology : CB, 20(13), 1227-1232. http://doi.org/10.1016/j.cub.2010.05.058

Botelho, S. C., Österberg, M., Reichert, A. S., Yamano, K., Björkholm, P., Endo, T., et al. (2011). TIM23-mediated insertion of transmembrane $\alpha$-helices into the mitochondrial inner membrane. The EMBO Journal, 30(6), 1003-1011. http://doi.org/10.1038/emboj.2011.29

Bömer, U., Meijer, M., Maarse, A. C., Hönlinger, A., Dekker, P. J., Pfanner, N., \& Rassow, J. (1997). Multiple interactions of components mediating preprotein translocation across the inner mitochondrial membrane. The EMBO Journal, 16(9), 2205-2216. http://doi.org/10.1093/emboj/16.9.2205 
Böttinger, L., Horvath, S. E., Kleinschroth, T., Hunte, C., Daum, G., Pfanner, N., \& Becker, T. (2012). Phosphatidylethanolamine and cardiolipin differentially affect the stability of mitochondrial respiratory chain supercomplexes. Journal of Molecular Biology, 423(5), 677-686. http://doi.org/10.1016/j.jmb.2012.09.001

Bradford, M. M. (1976). A rapid and sensitive method for the quantitation of microgram quantities of protein utilizing the principle of protein-dye binding. Analytical Biochemistry, 72, 248-254.

Brix, J., Rüdiger, S., Bukau, B., Schneider-Mergener, J., \& Pfanner, N. (1999). Distribution of binding sequences for the mitochondrial import receptors Tom20, Tom22, and Tom70 in a presequence-carrying preprotein and a noncleavable preprotein. Journal of Biological Chemistry, 274(23), 16522-16530.

Brunner, S., Everard-Gigot, V., \& Stuart, R. A. (2002). Su e of the yeast F1Fo-ATP synthase forms homodimers. Journal of Biological Chemistry, 277(50), 4848448489. http://doi.org/10.1074/jbc.M209382200

Chacinska, A., Koehler, C. M., Milenkovic, D., Lithgow, T., \& Pfanner, N. (2009). Importing mitochondrial proteins: machineries and mechanisms. Cell, 138(4), 628-644. http://doi.org/10.1016/j.cell.2009.08.005

Chance, B., \& Williams, G. R. (1955). A method for the localization of sites for oxidative phosphorylation. Nature, 176(4475), 250-254.

http://doi.org/10.1038/176250a0

Chen, Y.-C., Taylor, E. B., Dephoure, N., Heo, J.-M., Tonhato, A., Papandreou, I., et al. (2012). Identification of a protein mediating respiratory supercomplex stability. Cell Metabolism, 15(3), 348-360. http://doi.org/10.1016/j.cmet.2012.02.006

Christianson, T. W., Sikorski, R. S., Dante, M., Shero, J. H., \& Hieter, P. (1992). Multifunctional yeast high-copy-number shuttle vectors. Gene, 110(1), 119-122.

Claypool, S. M., Oktay, Y., Boontheung, P., Loo, J. A., \& Koehler, C. M. (2008). Cardiolipin defines the interactome of the major ADP/ATP carrier protein of the mitochondrial inner membrane. The Journal of Cell Biology, 182(5), 937-950. http://doi.org/10.1083/jcb.200801152

Cox, J. S., Chapman, R. E., \& Walter, P. (1997). The unfolded protein response coordinates the production of endoplasmic reticulum protein and endoplasmic reticulum membrane. Molecular Biology of the Cell, 8(9), 1805-1814.

Cruciat, C. M., Brunner, S., Baumann, F., Neupert, W., \& Stuart, R. A. (2000). The cytochrome bc1 and cytochrome c oxidase complexes associate to form a single supracomplex in yeast mitochondria. Journal of Biological Chemistry, 275(24), 18093-18098. http://doi.org/10.1074/jbc.M001901200 
Cui, T.-Z., Conte, A., Fox, J. L., Zara, V., \& Winge, D. R. (2014). Modulation of the respiratory supercomplexes in yeast: enhanced formation of cytochrome oxidase increases the stability and abundance of respiratory supercomplexes. The Journal of Biological Chemistry, 289(9), 6133-6141. http://doi.org/10.1074/jbc.M113.523688

Curran, B. P. G., \& Bugeja, V. (2006). Basic investigations in Saccharomyces cerevisiae. Methods in Molecular Biology (Clifton, N.J.), 313, 1-13. http://doi.org/10.1385/1-59259-958-3:001

D'Aurelio, M., Gajewski, C. D., Lenaz, G., \& Manfredi, G. (2006). Respiratory chain supercomplexes set the threshold for respiration defects in human mtDNA mutant cybrids. Human Molecular Genetics, 15(13), 2157-2169. http://doi.org/10.1093/hmg/ddl141

Daum, G., Böhni, P. C., \& Schatz, G. (1982). Import of proteins into mitochondria. Cytochrome b2 and cytochrome c peroxidase are located in the intermembrane space of yeast mitochondria. Journal of Biological Chemistry, 257(21), 1302813033.

Davis, A. J., Alder, N. N., Jensen, R. E., \& Johnson, A. E. (2007). The Tim9p/10p and Tim8p/13p complexes bind to specific sites on Tim23p during mitochondrial protein import. Molecular Biology of the Cell, 18(2), 475-486.

http://doi.org/10.1091/mbc.E06-06-0546

Dekker, P. J., Martin, F., Maarse, A. C., Bömer, U., Muller, H., Guiard, B., et al. (1997). The Tim core complex defines the number of mitochondrial translocation contact sites and can hold arrested preproteins in the absence of matrix Hsp70Tim44. The EMBO Journal, 16(17), 5408-5419. http://doi.org/10.1093/emboj/16.17.5408

Deshpande, A. P., \& Patel, S. S. (2012). Mechanism of transcription initiation by the yeast mitochondrial RNA polymerase. Biochimica Et Biophysica Acta, 1819(910), 930-938. http://doi.org/10.1016/j.bbagrm.2012.02.003

Dienhart, M. K., \& Stuart, R. A. (2008). The yeast Aac2 protein exists in physical association with the cytochrome bc1-COX supercomplex and the TIM23 machinery. Molecular Biology of the Cell, 19(9), 3934-3943. http://doi.org/10.1091/mbc.E08-04-0402

Dimmer, K. S., Papić, D., Schumann, B., Sperl, D., Krumpe, K., Walther, D. M., \& Rapaport, D. (2012). A crucial role for Mim2 in the biogenesis of mitochondrial outer membrane proteins. Journal of Cell Science, 125(Pt 14), 3464-3473. http://doi.org/10.1242/jcs.103804

Dudek, J., Rehling, P., \& van der Laan, M. (2013). Mitochondrial protein import: common principles and physiological networks. Biochimica Et Biophysica Acta, 1833(2), 274-285. http://doi.org/10.1016/j.bbamcr.2012.05.028 


\section{BIBLIOGRAPHY}

Ernster, L., \& Schatz, G. (1981). Mitochondria: a historical review. The Journal of Cell Biology, 91(3 Pt 2), 227s-255s.

Esser, K., Tursun, B., Ingenhoven, M., Michaelis, G., \& Pratje, E. (2002). A novel twostep mechanism for removal of a mitochondrial signal sequence involves the mAAA complex and the putative rhomboid protease Pcp1. Journal of Molecular Biology, 323(5), 835-843.

Falke, J. J., \& Koshland, D. E. (1987). Global flexibility in a sensory receptor: a sitedirected cross-linking approach. Science, 237(4822), 1596-1600.

Fox, T. D. (2012). Mitochondrial protein synthesis, import, and assembly. Genetics, 192(4), 1203-1234. http://doi.org/10.1534/genetics.112.141267

Fölsch, H., Guiard, B., Neupert, W., \& Stuart, R. A. (1996). Internal targeting signal of the BCS1 protein: a novel mechanism of import into mitochondria. The EMBO Journal, 15(3), 479-487.

Frazier, A. E., Chacinska, A., Truscott, K. N., Guiard, B., Pfanner, N., \& Rehling, P. (2003). Mitochondria use different mechanisms for transport of multispanning membrane proteins through the intermembrane space. Molecular and Cellular Biology, 23(21), 7818-7828. http://doi.org/10.1128/MCB.23.21.78187828.2003

Frazier, A. E., Taylor, R. D., Mick, D. U., Warscheid, B., Stoepel, N., Meyer, H. E., et al. (2006). Mdm38 interacts with ribosomes and is a component of the mitochondrial protein export machinery. The Journal of Cell Biology, 172(4), 553-564. http://doi.org/10.1083/jcb.200505060

Friedman, J. R., \& Nunnari, J. (2014). Mitochondrial form and function. Nature, 505(7483), 335-343. http://doi.org/10.1038/nature12985

Funes, S., Nargang, F. E., Neupert, W., \& Herrmann, J. M. (2004). The Oxa2 protein of Neurospora crassa plays a critical role in the biogenesis of cytochrome oxidase and defines a ubiquitous subbranch of the Oxa1/YidC/Alb3 protein family. Molecular Biology of the Cell, 15(4), 1853-1861. http://doi.org/10.1091/mbc.E03-11-0789

Gabaldón, T., \& Huynen, M. A. (2004). Shaping the mitochondrial proteome. Biochimica Et Biophysica Acta, 1659(2-3), 212-220. http://doi.org/10.1016/j.bbabio.2004.07.011

Gallagher, S., Winston, S. E., Fuller, S. A., \& Hurrell, J. G. R. (2004). Immunoblotting and immunodetection. Current Protocols in Molecular Biology / Edited by Frederick M. Ausubel ... [Et Al.], Chapter 10, Unit 10.8-10.8.24. http://doi.org/10.1002/0471142727.mb1008s66

Gaspard, G. J., \& McMaster, C. R. (2015). Cardiolipin metabolism and its causal role in the etiology of the inherited cardiomyopathy Barth syndrome. Chemistry and Physics of Lipids, 193, 1-10. http://doi.org/10.1016/j.chemphyslip.2015.09.005 
Gebert, M., Schrempp, S. G., Mehnert, C. S., Heißwolf, A. K., Oeljeklaus, S., Ieva, R., et al. (2012). Mgr2 promotes coupling of the mitochondrial presequence translocase to partner complexes. The Journal of Cell Biology, 197(5), 595-604. http://doi.org/10.1083/jcb.201110047

Gebert, N., Chacinska, A., Wagner, K., Guiard, B., Koehler, C. M., Rehling, P., et al. (2008). Assembly of the three small Tim proteins precedes docking to the mitochondrial carrier translocase. EMBO Reports, 9(6), 548-554. http://doi.org/10.1038/embor.2008.49

Gietz, R. D., \& Woods, R. A. (2002). Transformation of yeast by lithium acetate/single-stranded carrier DNA/polyethylene glycol method. Methods in Enzymology, 350, 87-96.

Glick, B. S., Brandt, A., Cunningham, K., Müller, S., Hallberg, R. L., \& Schatz, G. (1992). Cytochromes $\mathrm{c} 1$ and $\mathrm{b} 2$ are sorted to the intermembrane space of yeast mitochondria by a stop-transfer mechanism. Cell, 69(5), 809-822.

Grandier-Vazeille, X., Bathany, K., Chaignepain, S., Camougrand, N., Manon, S., \& Schmitter, J. M. (2001). Yeast mitochondrial dehydrogenases are associated in a supramolecular complex. Biochemistry, 40(33), 9758-9769. http://doi.org/10.1021/bi010277r

Green, D. R., \& Reed, J. C. (1998). Mitochondria and apoptosis. Science, 281(5381), 1309-1312.

Gruschke, S., Römpler, K., Hildenbeutel, M., Kehrein, K., Kühl, I., Bonnefoy, N., \& Ott, M. (2012). The Cbp3-Cbp6 complex coordinates cytochrome b synthesis with bc(1) complex assembly in yeast mitochondria. The Journal of Cell Biology, 199(1), 137-150. http://doi.org/10.1083/jcb.201206040

Hackenbrock, C. R., Chazotte, B., \& Gupte, S. S. (1986). The random collision model and a critical assessment of diffusion and collision in mitochondrial electron transport. Journal of Bioenergetics and Biomembranes, 18(5), 331-368.

Hackenbrock, C. R., Schneider, H., Lemasters, J. J., \& Höchli, M. (1980). Relationships between bilayer lipid, motional freedom of oxidoreductase components, and electron transfer in the mitochondrial inner membrane. Advances in Experimental Medicine and Biology, 132, 245-263.

Hanahan, D. (1983). Studies on transformation of Escherichia coli with plasmids. Journal of Molecular Biology, 166(4), 557-580.

Hartl, F. U., Ostermann, J., Guiard, B., \& Neupert, W. (1987). Successive translocation into and out of the mitochondrial matrix: targeting of proteins to the intermembrane space by a bipartite signal peptide. Cell, 51(6), 1027-1037. 
Hayashi, T., Asano, Y., Shintani, Y., Aoyama, H., Kioka, H., Tsukamoto, O., et al. (2015). Higd1a is a positive regulator of cytochrome c oxidase. Proceedings of the National Academy of Sciences of the United States of America, 112(5), 1553-1558. http://doi.org/10.1073/pnas.1419767112

Heinemeyer, J., Braun, H.-P., Boekema, E. J., \& Kouril, R. (2007). A structural model of the cytochrome $\mathrm{C}$ reductase/oxidase supercomplex from yeast mitochondria. Journal of Biological Chemistry, 282(16), 12240-12248. http://doi.org/10.1074/jbc.M610545200

Helbig, A. O., de Groot, M. J. L., van Gestel, R. A., Mohammed, S., de Hulster, E. A. F., Luttik, M. A. H., et al. (2009). A three-way proteomics strategy allows differential analysis of yeast mitochondrial membrane protein complexes under anaerobic and aerobic conditions. Proteomics, 9(20), 4787-4798.

http://doi.org/10.1002/pmic.200800951

Hell, K., Herrmann, J. M., Pratje, E., Neupert, W., \& Stuart, R. A. (1998). Oxa1p, an essential component of the $\mathrm{N}$-tail protein export machinery in mitochondria. Proceedings of the National Academy of Sciences, 95(5), 2250-2255.

Hell, K., Neupert, W., \& Stuart, R. A. (2001). Oxa1p acts as a general membrane insertion machinery for proteins encoded by mitochondrial DNA. The EMBO Journal, 20(6), 1281-1288. http://doi.org/10.1093/emboj/20.6.1281

Hell, K., Tzagoloff, A., Neupert, W., \& Stuart, R. A. (2000). Identification of Cox20p, a novel protein involved in the maturation and assembly of cytochrome oxidase subunit 2. Journal of Biological Chemistry, 275(7), 4571-4578.

Herlan, M., Vogel, F., Bornhovd, C., Neupert, W., \& Reichert, A. S. (2003). Processing of Mgm1 by the rhomboid-type protease Pcp1 is required for maintenance of mitochondrial morphology and of mitochondrial DNA. Journal of Biological Chemistry, 278(30), 27781-27788. http://doi.org/10.1074/jbc.M211311200

Herrmann, J. M., Neupert, W., \& Stuart, R. A. (1997). Insertion into the mitochondrial inner membrane of a polytopic protein, the nuclear-encoded Oxa1p. The EMBO Journal, 16(9), 2217-2226. http://doi.org/10.1093/emboj/16.9.2217

Hess, D. C., Myers, C. L., Huttenhower, C., Hibbs, M. A., Hayes, A. P., Paw, J., et al. (2009). Computationally driven, quantitative experiments discover genes required for mitochondrial biogenesis. PLoS Genetics, 5(3), e1000407. http://doi.org/10.1371/journal.pgen.1000407

Hewitt, V. L., Gabriel, K., \& Traven, A. (2014). The ins and outs of the intermembrane space: diverse mechanisms and evolutionary rewiring of mitochondrial protein import routes. Biochimica Et Biophysica Acta, 1840(4), 1246-1253.

http://doi.org/10.1016/j.bbagen.2013.08.013 
Hildebrand, P. W., Preissner, R., \& Frömmel, C. (2004). Structural features of transmembrane helices. FEBS Letters, 559(1-3), 145-151. http://doi.org/10.1016/S0014-5793(04)00061-4

Hofmann, K., \& Stoffel, W. (1993). TMbase - A database of Membrane Spanning Protein Segments. Biol. Chem. Hoppe-Seyler (Vol. 374, p. 166).

Hubbard, S. J. (1998). The structural aspects of limited proteolysis of native proteins. Biochimica Et Biophysica Acta, 1382(2), 191-206.

Hunte, C., Koepke, J., Lange, C., Rossmanith, T., \& Michel, H. (2000). Structure at 2.3 A resolution of the cytochrome bc(1) complex from the yeast Saccharomyces cerevisiae co-crystallized with an antibody Fv fragment. Structure (London, England : 1993), 8(6), 669-684.

Hutu, D. P., Guiard, B., Chacinska, A., Becker, D., Pfanner, N., Rehling, P., \& van der Laan, M. (2008). Mitochondrial protein import motor: differential role of Tim44 in the recruitment of Pam 17 and J-complex to the presequence translocase. Molecular Biology of the Cell, 19(6), 2642-2649. http://doi.org/10.1091/mbc.E07-12-1226

Janke, C., Magiera, M. M., Rathfelder, N., Taxis, C., Reber, S., Maekawa, H., et al. (2004). A versatile toolbox for PCR-based tagging of yeast genes: new fluorescent proteins, more markers and promoter substitution cassettes. Yeast (Chichester, England), 21(11), 947-962. http://doi.org/10.1002/yea.1142

Jia, L., Dienhart, M., Schramp, M., McCauley, M., Hell, K., \& Stuart, R. A. (2003). Yeast Oxa1 interacts with mitochondrial ribosomes: the importance of the C-terminal region of Oxa1. The EMBO Journal, 22(24), 6438-6447.

http://doi.org/10.1093/emboj/cdg624

Käser, M., \& Langer, T. (2000). Protein degradation in mitochondria. Seminars in Cell \& Developmental Biology, 11(3), 181-190. http://doi.org/10.1006/scdb.2000.0166

Kehrein, K., Bonnefoy, N., \& Ott, M. (2013). Mitochondrial protein synthesis: efficiency and accuracy. Antioxidants \& Redox Signaling, 19(16), 1928-1939. http://doi.org/10.1089/ars.2012.4896

Kemper, C., Habib, S. J., Engl, G., Heckmeyer, P., Dimmer, K. S., \& Rapaport, D. (2008). Integration of tail-anchored proteins into the mitochondrial outer membrane does not require any known import components. Journal of Cell Science, 121(Pt 12), 1990-1998. http://doi.org/10.1242/jcs.024034

Kitakawa, M., Graack, H. R., Grohmann, L., Goldschmidt-Reisin, S., Herfurth, E., Wittmann-Liebold, B., et al. (1997). Identification and characterization of the genes for mitochondrial ribosomal proteins of Saccharomyces cerevisiae. European Journal of Biochemistry / FEBS, 245(2), 449-456. 
Knop, M., Siegers, K., Pereira, G., Zachariae, W., Winsor, B., Nasmyth, K., \& Schiebel, E. (1999). Epitope tagging of yeast genes using a PCR-based strategy: more tags and improved practical routines. Yeast (Chichester, England), 15(10B), 963-972. http://doi.org/10.1002/(SICI)1097-0061(199907)15:10B<963::AIDYEA399>3.0.CO;2-W

Kobashi, K. (1968). Catalytic oxidation of sulfhydryl groups by o-phenanthroline copper complex. Biochimica Et Biophysica Acta, 158(2), 239-245.

Koppen, M., \& Langer, T. (2007). Protein degradation within mitochondria: versatile activities of AAA proteases and other peptidases. Critical Reviews in Biochemistry and Molecular Biology, 42(3), 221-242.

http://doi.org/10.1080/10409230701380452

Krumpe, K., Frumkin, I., Herzig, Y., Rimon, N., Özbalci, C., Brügger, B., et al. (2012).

Ergosterol content specifies targeting of tail-anchored proteins to mitochondrial outer membranes. Molecular Biology of the Cell, 23(20), 3927-3935.

http://doi.org/10.1091/mbc.E11-12-0994

Kutik, S., Stojanovski, D., Becker, L., Becker, T., Meinecke, M., Krüger, V., et al. (2008). Dissecting membrane insertion of mitochondrial beta-barrel proteins. Cell, 132(6), 1011-1024. http://doi.org/10.1016/j.cell.2008.01.028

Laemmli, U. K. (1970). Cleavage of structural proteins during the assembly of the head of bacteriophage T4. Nature, 227(5259), 680-685.

Lapuente-Brun, E., Moreno-Loshuertos, R., Acin-Perez, R., Latorre-Pellicer, A., Colás, C., Balsa, E., et al. (2013). Supercomplex assembly determines electron flux in the mitochondrial electron transport chain. Science, 340(6140), 1567-1570. http://doi.org/10.1126/science.1230381

Lee, C. M., Sedman, J., Neupert, W., \& Stuart, R. A. (1999). The DNA helicase, Hmi1p, is transported into mitochondria by a C-terminal cleavable targeting signal. Journal of Biological Chemistry, 274(30), 20937-20942.

Lenaz, G., \& Genova, M. L. (2009). Structural and functional organization of the mitochondrial respiratory chain: A dynamic super-assembly. The International Journal of Biochemistry \& Cell Biology, 41(10), 1750-1772.

http://doi.org/10.1016/j.biocel.2009.04.003

Lenaz, G., \& Genova, M. L. (2012). Supramolecular organisation of the mitochondrial respiratory chain: a new challenge for the mechanism and control of oxidative phosphorylation. Advances in Experimental Medicine and Biology, 748, 107-144. http://doi.org/10.1007/978-1-4614-3573-0_5

Levchenko, M., Wuttke, J.-M., Römpler, K., Schmidt, B., Neifer, K., Juris, L., et al. (2016). Cox26 is a novel stoichiometric subunit of the yeast cytochrome $\mathrm{c}$ oxidase. BBA - Molecular Cell Research, 1-35.

http://doi.org/10.1016/j.bbamcr.2016.04.007 
Lill, R., Hoffmann, B., Molik, S., Pierik, A. J., Rietzschel, N., Stehling, O., et al. (2012). The role of mitochondria in cellular iron-sulfur protein biogenesis and iron metabolism. Biochimica Et Biophysica Acta, 1823(9), 1491-1508. http://doi.org/10.1016/j.bbamcr.2012.05.009

Longtine, M. S., McKenzie, A., Demarini, D. J., Shah, N. G., Wach, A., Brachat, A., et al. (1998). Additional modules for versatile and economical PCR-based gene deletion and modification in Saccharomyces cerevisiae. Yeast (Chichester, England), 14(10), 953-961. http://doi.org/10.1002/(SICI)10970061(199807)14:10<953::AID-YEA293>3.0.CO;2-U

Maréchal, A., Meunier, B., Lee, D., Orengo, C., \& Rich, P. R. (2012). Yeast cytochrome c oxidase: a model system to study mitochondrial forms of the haem-copper oxidase superfamily. Biochimica Et Biophysica Acta, 1817(4), 620-628. http://doi.org/10.1016/j.bbabio.2011.08.011

Margulis, L. (1970). Origin of Eukaryotic Cells. New Haven, CT: Yale University Press.

McQuibban, G. A., Saurya, S., \& Freeman, M. (2003). Mitochondrial membrane remodelling regulated by a conserved rhomboid protease. Nature, 423(6939), 537-541. http://doi.org/10.1038/nature01633

McStay, G. P., Su, C.-H., \& Tzagoloff, A. (2013). Modular assembly of yeast cytochrome oxidase. Molecular Biology of the Cell, 24(4), 440-452.

http://doi.org/10.1091/mbc.E12-10-0749

Meier, S., Neupert, W., \& Herrmann, J. M. (2005). Proline residues of transmembrane domains determine the sorting of inner membrane proteins in mitochondria. The Journal of Cell Biology, 170(6), 881-888.

http://doi.org/10.1083/jcb.200505126

Meisinger, C., Pfanner, N., \& Truscott, K. N. (2006). Isolation of yeast mitochondria. Methods in Molecular Biology (Clifton, N.J.), 313, 33-39. http://doi.org/10.1385/1-59259-958-3:033

Mick, D. U., Fox, T. D., \& Rehling, P. (2011). Inventory control: cytochrome c oxidase assembly regulates mitochondrial translation. Nature Reviews. Molecular Cell Biology, 12(1), 14-20. http://doi.org/10.1038/nrm3029

Mick, D. U., Vukotic, M., Piechura, H., Meyer, H. E., Warscheid, B., Deckers, M., \& Rehling, P. (2010). Coa3 and Cox14 are essential for negative feedback regulation of COX1 translation in mitochondria. The Journal of Cell Biology, 191(1), 141-154. http://doi.org/10.1083/jcb.201007026

Mick, D. U., Wagner, K., van der Laan, M., Frazier, A. E., Perschil, I., Pawlas, M., et al. (2007). Shy1 couples Cox1 translational regulation to cytochrome c oxidase assembly. The EMBO Journal, 26(20), 4347-4358.

http://doi.org/10.1038/sj.emboj.7601862 
Mileykovskaya, E., Penczek, P. A., Fang, J., Mallampalli, V. K. P. S., Sparagna, G. C., \& Dowhan, W. (2012). Arrangement of the respiratory chain complexes in Saccharomyces cerevisiae supercomplex III2IV2 revealed by single particle cryoelectron microscopy. The Journal of Biological Chemistry, 287(27), 23095-23103. http://doi.org/10.1074/jbc.M112.367888

Mishra, P., \& Chan, D. C. (2014). Mitochondrial dynamics and inheritance during cell division, development and disease. Nature Reviews. Molecular Cell Biology, 15(10), 634-646. http://doi.org/10.1038/nrm3877

Moreno-Lastres, D., Fontanesi, F., García-Consuegra, I., Martín, M. A., Arenas, J., Barrientos, A., \& Ugalde, C. (2012). Mitochondrial complex I plays an essential role in human respirasome assembly. Cell Metabolism, 15(3), 324-335. http://doi.org/10.1016/j.cmet.2012.01.015

Moualij, el, B., Duyckaerts, C., Lamotte-Brasseur, J., \& Sluse, F. E. (1997). Phylogenetic classification of the mitochondrial carrier family of Saccharomyces cerevisiae. Yeast (Chichester, England), 13(6), 573-581. http://doi.org/10.1002/(SICI)1097-0061(199705)13:6<573::AIDYEA107>3.0.CO;2-I

Mourier, A., Matic, S., Ruzzenente, B., Larsson, N.-G., \& Milenkovic, D. (2014). The respiratory chain supercomplex organization is independent of COX7a2l isoforms. Cell Metabolism, 20(6), 1069-1075. http://doi.org/10.1016/j.cmet.2014.11.005

Müller, M., Lu, K., \& Reichert, A. S. (2015). Mitophagy and mitochondrial dynamics in Saccharomyces cerevisiae. Biochimica Et Biophysica Acta, 1853(10 Pt B), 27662774. http://doi.org/10.1016/j.bbamcr.2015.02.024

Nelson, D. R., Felix, C. M., \& Swanson, J. M. (1998). Highly conserved charge-pair networks in the mitochondrial carrier family. Journal of Molecular Biology, 277(2), 285-308. http://doi.org/10.1006/jmbi.1997.1594

Nolden, M., Ehses, S., Koppen, M., Bernacchia, A., Rugarli, E. I., \& Langer, T. (2005). The m-AAA protease defective in hereditary spastic paraplegia controls ribosome assembly in mitochondria. Cell, 123(2), 277-289. http://doi.org/10.1016/j.cell.2005.08.003

Ott, M., \& Herrmann, J. M. (2010). Co-translational membrane insertion of mitochondrially encoded proteins. Biochimica Et Biophysica Acta, 1803(6), 767775. http://doi.org/10.1016/j.bbamcr.2009.11.010

Panov, A., Dikalov, S., Shalbuyeva, N., Hemendinger, R., Greenamyre, J. T., \& Rosenfeld, J. (2007). Species- and tissue-specific relationships between mitochondrial permeability transition and generation of ROS in brain and liver mitochondria of rats and mice. American Journal of Physiology. Cell Physiology, 292(2), C708-18. http://doi.org/10.1152/ajpcell.00202.2006 
Park, K., Botelho, S. C., Hong, J., Österberg, M., \& Kim, H. (2013). Dissecting stop transfer versus conservative sorting pathways for mitochondrial inner membrane proteins in vivo. The Journal of Biological Chemistry, 288(3), 15211532. http://doi.org/10.1074/jbc.M112.409748

Paumard, P., Vaillier, J., Coulary, B., Schaeffer, J., Soubannier, V., Mueller, D. M., et al. (2002). The ATP synthase is involved in generating mitochondrial cristae morphology. The EMBO Journal, 21(3), 221-230.

http://doi.org/10.1093/emboj/21.3.221

Pfanner, N., Hoeben, P., Tropschug, M., \& Neupert, W. (1987). The carboxyl-terminal two-thirds of the ADP/ATP carrier polypeptide contains sufficient information to direct translocation into mitochondria. Journal of Biological Chemistry, 262(31), 14851-14854.

Pfeffer, S., Woellhaf, M. W., Herrmann, J. M., \& Förster, F. (2015). Organization of the mitochondrial translation machinery studied in situ by cryoelectron tomography. Nature Communications, 6, 6019. http://doi.org/10.1038/ncomms7019

Pfeiffer, K., Gohil, V., Stuart, R. A., Hunte, C., Brandt, U., Greenberg, M. L., \& Schägger, H. (2003). Cardiolipin stabilizes respiratory chain supercomplexes. Journal of Biological Chemistry, 278(52), 52873-52880. http://doi.org/10.1074/jbc.M308366200

Pratje, E., Mannhaupt, G., Michaelis, G., \& Beyreuther, K. (1983). A nuclear mutation prevents processing of a mitochondrially encoded membrane protein in Saccharomyces cerevisiae. The EMBO Journal, 2(7), 1049-1054.

Preuss, M., Leonhard, K., Hell, K., Stuart, R. A., Neupert, W., \& Herrmann, J. M. (2001). Mba1, a novel component of the mitochondrial protein export machinery of the yeast Saccharomyces cerevisiae. The Journal of Cell Biology, 153(5), 1085-1096.

Prokisch, H., Scharfe, C., Camp, D. G., Xiao, W., David, L., Andreoli, C., et al. (2004). Integrative analysis of the mitochondrial proteome in yeast. PLoS Biology, 2(6), e160. http://doi.org/10.1371/journal.pbio.0020160

Rak, M., Zeng, X., Brière, J.-J., \& Tzagoloff, A. (2009). Assembly of F0 in Saccharomyces cerevisiae. Biochimica Et Biophysica Acta, 1793(1), 108-116. http://doi.org/10.1016/j.bbamcr.2008.07.001

Rehling, P., Brandner, K., \& Pfanner, N. (2004). Mitochondrial import and the twinpore translocase. Nature Reviews. Molecular Cell Biology, 5(7), 519-530. http://doi.org/10.1038/nrm1426

Rehling, P., Model, K., Brandner, K., Kovermann, P., Sickmann, A., Meyer, H. E., et al. (2003). Protein insertion into the mitochondrial inner membrane by a twin-pore translocase. Science, 299(5613), 1747-1751. http://doi.org/10.1126/science.1080945 
Reinhold, R., Krüger, V., Meinecke, M., Schulz, C., Schmidt, B., Grunau, S. D., et al. (2012). The channel-forming Sym1 protein is transported by the TIM23 complex in a presequence-independent manner. Molecular and Cellular Biology, 32(24), 5009-5021. http://doi.org/10.1128/MCB.00843-12

Rimessi, A., Giorgi, C., Pinton, P., \& Rizzuto, R. (2008). The versatility of mitochondrial calcium signals: from stimulation of cell metabolism to induction of cell death. Biochimica Et Biophysica Acta, 1777(7-8), 808-816. http://doi.org/10.1016/j.bbabio.2008.05.449

Roberts, M. J., Bentley, M. D., \& Harris, J. M. (2002). Chemistry for peptide and protein PEGylation. Advanced Drug Delivery Reviews, 54(4), 459-476. http://doi.org/10.1016/j.addr.2012.09.025

Roise, D., Horvath, S. J., Tomich, J. M., Richards, J. H., \& Schatz, G. (1986). A chemically synthesized pre-sequence of an imported mitochondrial protein can form an amphiphilic helix and perturb natural and artificial phospholipid bilayers. The EMBO Journal, 5(6), 1327-1334.

Rojo, E. E., Stuart, R. A., \& Neupert, W. (1995). Conservative sorting of F0-ATPase subunit 9: export from matrix requires delta $\mathrm{pH}$ across inner membrane and matrix ATP. The EMBO Journal, 14(14), 3445-3451.

Rowland, A. A., \& Voeltz, G. K. (2012). Endoplasmic reticulum-mitochondria contacts: function of the junction. Nature Reviews. Molecular Cell Biology, 13(10), 607-625. http://doi.org/10.1038/nrm3440

Römpler, K., Juris, L., Wissel, M., Vukotic, M., Hofmann, K., \& Deckers, M. (currently under revision) The Rcf2 homologue Rcf3 associates with respiratory chain supercomplexes. The Journal of Biological Chemistry.

Rujiviphat, J., Wong, M. K., Won, A., Shih, Y.-L., Yip, C. M., \& McQuibban, G. A. (2015). Mitochondrial Genome Maintenance 1 (Mgm1) Protein Alters Membrane Topology and Promotes Local Membrane Bending. Journal of Molecular Biology, 427(16), 2599-2609. http://doi.org/10.1016/j.jmb.2015.03.006

Ryan, M. T., Voos, W., \& Pfanner, N. (2001). Assaying protein import into mitochondria. Methods in Cell Biology, 65, 189-215.

Sambrook, J., \& Russell, D. W. (2001). Molecular Cloning: A Laboratory Manual.

Saracco, S. A., \& Fox, T. D. (2002). Cox18p is required for export of the mitochondrially encoded Saccharomyces cerevisiae Cox2p C-tail and interacts with Pnt1p and Mss2p in the inner membrane. Molecular Biology of the Cell, 13(4), 1122-1131. http://doi.org/10.1091/mbc.01-12-0580

Saraste, M. (1999). Oxidative phosphorylation at the fin de siècle. Science, 283(5407), 1488-1493. 
Schatz, G., \& Dobberstein, B. (1996). Common principles of protein translocation across membranes. Science, 271(5255), 1519-1526.

Schäfer, E., Seelert, H., Reifschneider, N. H., Krause, F., Dencher, N. A., \& Vonck, J. (2006). Architecture of active mammalian respiratory chain supercomplexes. Journal of Biological Chemistry, 281(22), 15370-15375. http://doi.org/10.1074/jbc.M513525200

Schägger, H. (2001). Respiratory chain supercomplexes. IUBMB Life, 52(3-5), 119128. http://doi.org/10.1080/15216540152845911

Schägger, H. (2006). Tricine-SDS-PAGE. Nature Protocols, 1(1), 16-22. http://doi.org/10.1038/nprot.2006.4

Schägger, H., \& Jagow, von, G. (1991). Blue native electrophoresis for isolation of membrane protein complexes in enzymatically active form. Analytical Biochemistry, 199(2), 223-231.

Schägger, H., \& Pfeiffer, K. (2000). Supercomplexes in the respiratory chains of yeast and mammalian mitochondria. The EMBO Journal, 19(8), 1777-1783. http://doi.org/10.1093/emboj/19.8.1777

Schägger, H., de Coo, R., Bauer, M. F., Hofmann, S., Godinot, C., \& Brandt, U. (2004). Significance of respirasomes for the assembly/stability of human respiratory chain complex I. Journal of Biological Chemistry, 279(35), 36349-36353. http://doi.org/10.1074/jbc.M404033200

Schneider, A., Behrens, M., Scherer, P., Pratje, E., Michaelis, G., \& Schatz, G. (1991). Inner membrane protease $I$, an enzyme mediating intramitochondrial protein sorting in yeast. The EMBO Journal, 10(2), 247-254.

Schulz, C., Lytovchenko, O., Melin, J., Chacinska, A., Guiard, B., Neumann, P., et al. (2011). Tim50's presequence receptor domain is essential for signal driven transport across the TIM23 complex. The Journal of Cell Biology, 195(4), 643656. http://doi.org/10.1083/jcb.201105098

Schulz, C., Schendzielorz, A., \& Rehling, P. (2015). Unlocking the presequence import pathway. Trends in Cell Biology, 25(5), 265-275.

http://doi.org/10.1016/j.tcb.2014.12.001

Seelert, H., Dani, D. N., Dante, S., Hauß, T., Krause, F., Schäfer, E., et al. (2009). From protons to OXPHOS supercomplexes and Alzheimer's disease: structuredynamics-function relationships of energy-transducing membranes. Biochimica Et Biophysica Acta, 1787(6), 657-671. http://doi.org/10.1016/j.bbabio.2009.02.028 
Sesaki, H., Southard, S. M., Hobbs, A. E. A., \& Jensen, R. E. (2003). Cells lacking Pcp1p/Ugo2p, a rhomboid-like protease required for Mgm1p processing, lose mtDNA and mitochondrial structure in a Dnm1p-dependent manner, but remain competent for mitochondrial fusion. Biochemical and Biophysical Research Communications, 308(2), 276-283.

Sickmann, A., Reinders, J., Wagner, Y., Joppich, C., Zahedi, R., Meyer, H. E., et al. (2003). The proteome of Saccharomyces cerevisiae mitochondria. Proceedings of the National Academy of Sciences, 100(23), 13207-13212.

http://doi.org/10.1073/pnas.2135385100

Sikorski, R. S., \& Hieter, P. (1989). A system of shuttle vectors and yeast host strains designed for efficient manipulation of DNA in Saccharomyces cerevisiae. Genetics, 122(1), 19-27.

Simon, M., \& Faye, G. (1984). Steps in processing of the mitochondrial cytochrome oxidase subunit I pre-mRNA affected by a nuclear mutation in yeast. Proceedings of the National Academy of Sciences, 81(1), 8-12.

Sirrenberg, C., Endres, M., Fölsch, H., Stuart, R. A., Neupert, W., \& Brunner, M. (1998). Carrier protein import into mitochondria mediated by the intermembrane proteins Tim10/Mrs11 and Tim12/Mrs5. Nature, 391(6670), 912-915. http://doi.org/10.1038/36136

Soto, I. C., Fontanesi, F., Liu, J., \& Barrientos, A. (2012). Biogenesis and assembly of eukaryotic cytochrome c oxidase catalytic core. Biochimica Et Biophysica Acta, 1817(6), 883-897. http://doi.org/10.1016/j.bbabio.2011.09.005

Stiller, S. B., Höpker, J., Oeljeklaus, S., Schütze, C., Schrempp, S. G., Vent-Schmidt, J., et al. (2016). Mitochondrial OXA Translocase Plays a Major Role in Biogenesis of Inner-Membrane Proteins. Cell Metabolism, 23(5), 901-908. http://doi.org/10.1016/j.cmet.2016.04.005

Strecker, V., Kadeer, Z., Heidler, J., Cruciat, C. M., Angerer, H., Giese, H., et al. (2016). Supercomplex-associated Cox 26 protein binds to cytochrome c oxidase. Biochimica Et Biophysica Acta, 1863(7), 1643-1652. http://doi.org/10.1016/j.bbamcr.2016.04.012

Strisovsky, K., Sharpe, H. J., \& Freeman, M. (2009). Sequence-specific intramembrane proteolysis: identification of a recognition motif in rhomboid substrates. Molecular Cell, 36(6), 1048-1059. http://doi.org/10.1016/j.molcel.2009.11.006

Strogolova, V., Furness, A., Robb-McGrath, M., Garlich, J., \& Stuart, R. A. (2012). Rcf1 and Rcf2, members of the hypoxia-induced gene 1 protein family, are critical components of the mitochondrial cytochrome bc1-cytochrome c oxidase supercomplex. Molecular and Cellular Biology, 32(8), 1363-1373. http://doi.org/10.1128/MCB.06369-11 
Szklarczyk, R., \& Huynen, M. A. (2010). Mosaic origin of the mitochondrial proteome. Proteomics, 10(22), 4012-4024. http://doi.org/10.1002/pmic.201000329

Szyrach, G., Ott, M., Bonnefoy, N., Neupert, W., \& Herrmann, J. M. (2003). Ribosome binding to the Oxa1 complex facilitates co-translational protein insertion in mitochondria. The EMBO Journal, 22(24), 6448-6457.

http://doi.org/10.1093/emboj/cdg623

Tatsuta, T., Augustin, S., Nolden, M., Friedrichs, B., \& Langer, T. (2007). m-AAA protease-driven membrane dislocation allows intramembrane cleavage by rhomboid in mitochondria. The EMBO Journal, 26(2), 325-335. http://doi.org/10.1038/sj.emboj.7601514

Trumpower, B. L. (1990). Cytochrome bc1 complexes of microorganisms. Microbiological Reviews, 54(2), 101-129.

Truscott, K. N., Wiedemann, N., Rehling, P., Müller, H., Meisinger, C., Pfanner, N., \& Guiard, B. (2002). Mitochondrial import of the ADP/ATP carrier: the essential TIM complex of the intermembrane space is required for precursor release from the TOM complex. Molecular and Cellular Biology, 22(22), 7780-7789. http://doi.org/10.1128/MCB.22.22.7780-7789.2002

Tsukihara, T., Aoyama, H., Yamashita, E., Tomizaki, T., Yamaguchi, H., Shinzawa-Itoh, K., et al. (1995). Structures of metal sites of oxidized bovine heart cytochrome c oxidase at 2.8 A. Science, 269(5227), 1069-1074.

Tsukihara, T., Aoyama, H., Yamashita, E., Tomizaki, T., Yamaguchi, H., Shinzawa-Itoh, K., et al. (1996). The whole structure of the 13-subunit oxidized cytochrome c oxidase at 2.8 A. Science, 272(5265), 1136-1144.

Turakhiya, U., Malsburg, von der, K., Gold, V. A. M., Guiard, B., Chacinska, A., van der Laan, M., \& Ieva, R. (2016). Protein Import by the Mitochondrial Presequence Translocase in the Absence of a Membrane Potential. Journal of Molecular Biology. http://doi.org/10.1016/j.jmb.2016.01.020

Tyndall, J. D. A., Nall, T., \& Fairlie, D. P. (2005). Proteases universally recognize beta strands in their active sites. Chemical Reviews, 105(3), 973-999. http://doi.org/10.1021/cr040669e

Umezawa, H., Aoyagi, T., Morishima, H., Matsuzaki, M., \& Hamada, M. (1970). Pepstatin, a new pepsin inhibitor produced by Actinomycetes. The Journal of Antibiotics, 23(5), 259-262.

Urban, S. (2006). Rhomboid proteins: conserved membrane proteases with divergent biological functions. Genes \& Development, 20(22), 3054-3068. http://doi.org/10.1101/gad.1488606 
Urban, S., \& Freeman, M. (2003). Substrate specificity of rhomboid intramembrane proteases is governed by helix-breaking residues in the substrate transmembrane domain. Molecular Cell, 11(6), 1425-1434. http://doi.org/10.1016/S1097-2765(03)00181-3

van der Laan, M., Bohnert, M., Wiedemann, N., \& Pfanner, N. (2012). Role of MINOS in mitochondrial membrane architecture and biogenesis. Trends in Cell Biology, 22(4), 185-192. http://doi.org/10.1016/j.tcb.2012.01.004

van der Laan, M., Meinecke, M., Dudek, J., Hutu, D. P., Lind, M., Perschil, I., et al. (2007). Motor-free mitochondrial presequence translocase drives membrane integration of preproteins. Nature Cell Biology, 9(10), 1152-1159. http://doi.org/10.1038/ncb1635

van der Laan, M., Wiedemann, N., Mick, D. U., Guiard, B., Rehling, P., \& Pfanner, N. (2006). A role for Tim 21 in membrane-potential-dependent preprotein sorting in mitochondria. Current Biology : CB, 16(22), 2271-2276. http://doi.org/10.1016/j.cub.2006.10.025

Vögtle, F. N., Burkhart, J. M., Rao, S., Gerbeth, C., Hinrichs, J., Martinou, J.-C., et al. (2012). Intermembrane space proteome of yeast mitochondria. Molecular \& Cellular Proteomics : MCP, 11(12), 1840-1852. http://doi.org/10.1074/mcp.M112.021105

Vögtle, F. N., Wortelkamp, S., Zahedi, R. P., Becker, D., Leidhold, C., Gevaert, K., et al. (2009). Global analysis of the mitochondrial N-proteome identifies a processing peptidase critical for protein stability. Cell, 139(2), 428-439. http://doi.org/10.1016/j.cell.2009.07.045

Vukotic, M., Oeljeklaus, S., Wiese, S., Vögtle, F. N., Meisinger, C., Meyer, H. E., et al. (2012). Rcf1 mediates cytochrome oxidase assembly and respirasome formation, revealing heterogeneity of the enzyme complex. Cell Metabolism, 15(3), 336-347. http://doi.org/10.1016/j.cmet.2012.01.016

Wagener, N., Ackermann, M., Funes, S., \& Neupert, W. (2011). A pathway of protein translocation in mitochondria mediated by the AAA-ATPase Bcs1. Molecular Cell, 44(2), 191-202. http://doi.org/10.1016/j.molcel.2011.07.036

Wenz, T., Hielscher, R., Hellwig, P., Schägger, H., Richers, S., \& Hunte, C. (2009). Role of phospholipids in respiratory cytochrome bc(1) complex catalysis and supercomplex formation. Biochimica Et Biophysica Acta, 1787(6), 609-616. http://doi.org/10.1016/j.bbabio.2009.02.012

Wiedemann, N., van der Laan, M., Hutu, D. P., Rehling, P., \& Pfanner, N. (2007). Sorting switch of mitochondrial presequence translocase involves coupling of motor module to respiratory chain. The Journal of Cell Biology, 179(6), 11151122. http://doi.org/10.1083/jcb.200709087 
Ye, J., Davé, U. P., Grishin, N. V., Goldstein, J. L., \& Brown, M. S. (2000). Asparagineproline sequence within membrane-spanning segment of SREBP triggers intramembrane cleavage by site-2 protease. Proceedings of the National Academy of Sciences, 97(10), 5123-5128.

Yoshida, M., Muneyuki, E., \& Hisabori, T. (2001). ATP synthase--a marvellous rotary engine of the cell. Nature Reviews. Molecular Cell Biology, 2(9), 669-677. http://doi.org/10.1038/35089509

Zara, V., Conte, L., \& Trumpower, B. L. (2009). Biogenesis of the yeast cytochrome bc1 complex. Biochimica Et Biophysica Acta, 1793(1), 89-96. http://doi.org/10.1016/j.bbamcr.2008.04.011

Zeng, F. Y., Hopp, A., Soldner, A., \& Wess, J. (1999). Use of a disulfide cross-linking strategy to study muscarinic receptor structure and mechanisms of activation. Journal of Biological Chemistry, 274(23), 16629-16640.

Zhang, M., Mileykovskaya, E., \& Dowhan, W. (2002). Gluing the respiratory chain together. Cardiolipin is required for supercomplex formation in the inner mitochondrial membrane. Journal of Biological Chemistry, 277(46), 4355343556. http://doi.org/10.1074/jbc.C200551200

Zhang, M., Mileykovskaya, E., \& Dowhan, W. (2005). Cardiolipin is essential for organization of complexes III and IV into a supercomplex in intact yeast mitochondria. Journal of Biological Chemistry, 280(33), 29403-29408. http://doi.org/10.1074/jbc.M504955200

Zick, M., Duvezin-Caubet, S., Schäfer, A., Vogel, F., Neupert, W., \& Reichert, A. S. (2009). Distinct roles of the two isoforms of the dynamin-like GTPase Mgm1 in mitochondrial fusion. FEBS Letters, 583(13), 2237-2243.

http://doi.org/10.1016/j.febslet.2009.05.053 


\section{Acknowledgments}

This work had not been possible without the constant support and advice of the colleagues and friends around me. Therefore I would like to thank...

... Peter Rehling for the opportunity to work in his lab, for his supervision and advice. By offering project and position he enabled me to stay within the fascinating field of mitochondrial research and to strengthen my biochemical knowledge and skills.

... Heike Krebber and Dörthe Katschinski, for helpful discussions and motivating words during my thesis committee meetings. Along the same lines, Stefan Jakobs, Markus Bohnsack and Michael Meinecke for joining my extended committee for the examination.

... Markus Deckers for being my "supervisor-at-the-bench". His plethora of ideas was overwhelming at first but helped to overcome many an obstacle in my projects. I am very grateful for reams of scientific and personal discussions and his patience in the difficult and frustrating moments.

... All my collaborators in the different projects, even though some did not make it into this manuscript. Nora Vögtle and Ralf Zerbes for help with the protease screen, oxygen consumption measurements and large pore BN gels. Kay Hofmann for the Rcf alignments. Blanche Schwappach-Pignataro and Anne Clancy for support and assistance with SGR. Olaf Bernhard but also the group of Henning Urlaub for mass spectrometric analyses. Klaus Neifer for gel filtration analysis and Mirjam Wissel for great technical assistance in general.

... All current and former members of the Institute for Cellular Biochemistry for sharing scientific expertise and for making daily routine in the lab enjoyable. I could surely write a word about everyone but that unfortunately goes beyond the scope of this paragraph. Nevertheless, special thank goes to Sylvie, David, Alex and Markus for critically reading my manuscript and to Moritz, Mirjam, Tobi, Abhishek and David for reliably making me laugh!

... My family and my friends for open ears and arms at any time.

... Til for finding the right words in times when mine don't make sense and ...

... the universe ;-) 